\title{
(1)
}

Universidad Peruana de Ciencias Aplicadas

FACULTAD DE INGENIERÍA

PROGRAMA ACADÉMICO DE INGENIERÍA DE SISTEMAS DE

INFORMACIÓN

\section{MODELO DE MIGRACIÓN A LA NUBE DE LOS SERVIDORES DE UN DATA CENTER}

\author{
TESIS \\ Para optar el título profesional de Ingeniero de Sistemas de Información
}

\author{
Autores \\ Loo Cuya, Fabiola Magaly (0000-0002-1199-5221)
}

Rojas Solorzano, Christian Gianfranco (0000-0002-0473-463)

Asesor

Rivas Galloso, Paul Harry (0000-0003-1879-4833)

Lima, Noviembre 2018 
Esta tesis esta dedicada a mi familia, quienes me apoyaron de manera incondicional durante mi vida universitaria.

-Christian Rojas

Esta tesis esta dedicada a mi familia y amigos que me alentaron a seguir adelante a pesar de las caidas. A ti $H$, por apoyarme en esta aventura de la vida. -Fabiola Loo 


\section{Resumen}

El proyecto titulado Modelo de Migración a la Nube de los Servidores de un Data Center, tiene como objetivo principal implementar un modelo de migración a la nube de servidores de un data center de las pymes. Basado en un análisis de buenas prácticas y tecnologias de plataforma cloud. Ya que no todo tiene que migrarse a la nube, sino que esto depende de la necesidad del negocio. La propuesta está basada en las buenas prácticas que nos brindan los proveedores, la literatura y en los frameworks: Togaf y CCRA v4.

El proyecto consta de 3 partes: Input, solución y output.

El input está conformado por lo que representa el levantamiento de información y los business principles, goals and drivers que permiten conocer el negocio y sus activos y arquitectura de TI, y además por los requerimientos para la migración.

En base a lo antes relevado se evalúan tanto a la viabilidad de la migración como a los proveedores de plataforma cloud. Se determinan si es conveniente realizar o no la migración y que proveedor utilizar, entre las que se encuentran el repositorio cloud, el servicio importer de la plataforma, los scripts de comandos que permiten invocar los servicios, las instancias que son desplegadas en el ambiente cloud y la herramienta de administración y monitoreo sobre dichas instancias.

Por último, como output se obtienen los servidores en cloud, correctamente configurados.

Palabras Clave : data center ; migracion a la nube ; cloud computing ; servidores. 


\begin{abstract}
The main objective of the project entitled Migration Model to the Cloud of Servers in a Data Center is to implement a migration model to the cloud of servers in a data center of SMEs. Based on an analysis of good practices and cloud platform technologies. Since not everything has to migrate to the cloud, but this depends on the need of the business. The proposal is based on good practices provided by suppliers, literature and frameworks: Togaf and CCRA v4.
\end{abstract}

The project consists of 3 parts: Input, solution and output.

The input is made up of what represents the gathering of information and the business principles, goals and drivers that allow knowing the business and its assets and IT architecture, and also the requirements for migration.

Based on what was previously surveyed, both the viability of the migration and the cloud platform providers are evaluated. Determine if it is convenient to make or not the migration and which provider to use, among which are the cloud repository, the importer service of the platform, the command scripts that allow invoking the services, the instances that are deployed in the cloud environment and the administration and monitoring tool on these instances.

Finally, as output, servers are obtained in the cloud, correctly configured to avoid connection or other problems depending on the services they execute and the implementation document detailing the steps taken and the final configuration.

Key Words : data center ; migration to cloud ; cloud computing ; servers. 


\section{Contenido}

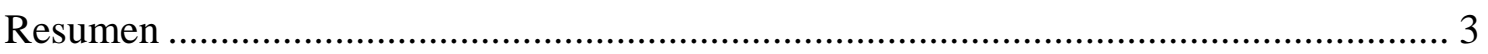

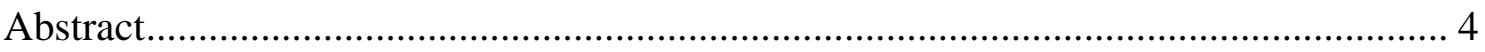

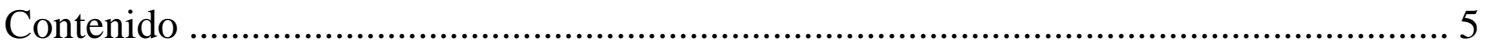

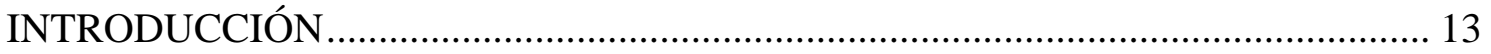

CAPÍTULO 1: DESCRIPCIÓN DEL PROYECTO …................................................... 15

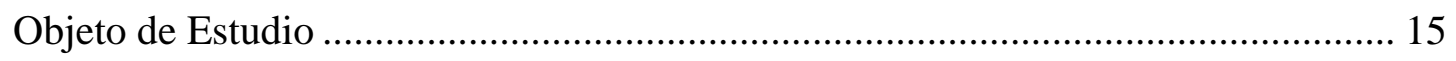

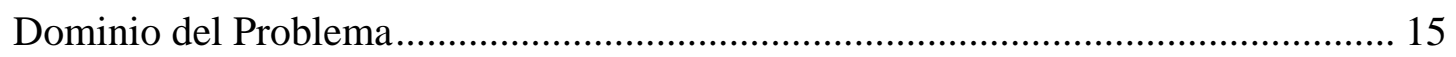

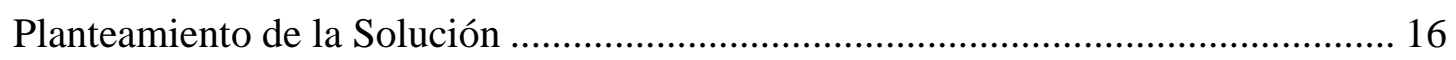

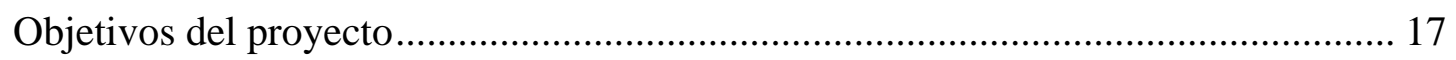

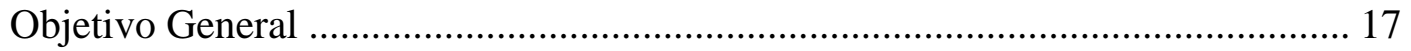

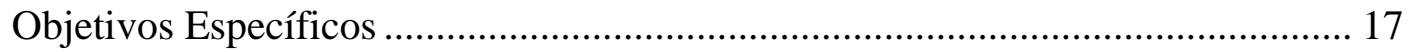

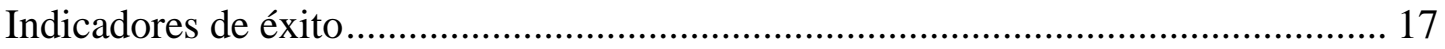

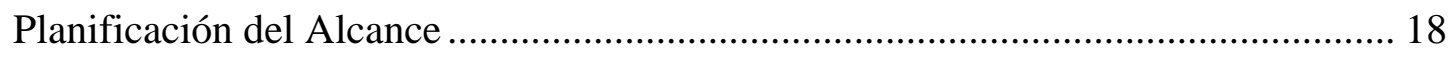

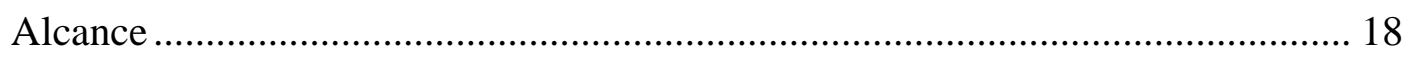

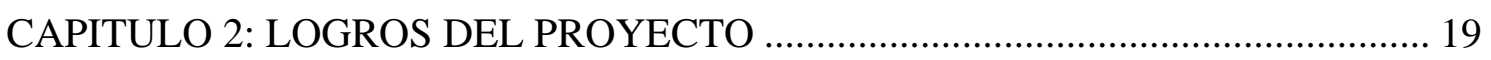

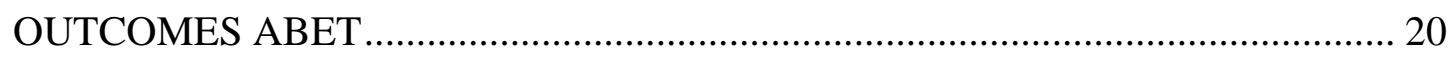

A Aplica conocimientos de matemáticas, ciencias, computación e ingeniería ...... 20

B Diseña y conduce experimentos en base al análisis e interpretación de datos.... 20

C Diseña sistemas, componentes o procesos para encontrar soluciones en la atención de necesidades teniendo en cuenta restricciones económicas, sociales, políticas, éticas de salud y seguridad y otras propias del entorno empresarial ..................... 20

D Participa en equipos multidisciplinarios desarrollando sus tareas con profesionales de diferentes especialidades o dominios de aplicación ........................................ 21

E Identifica, formula y resuelve problemas de Ingeniería ................................... 22

F Propone soluciones a problemas de Ingeniería con responsabilidad profesional y

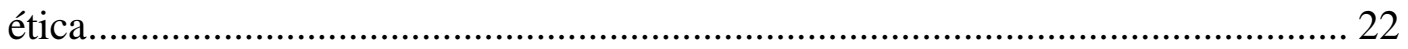

G Comunica ideas o resultados de manera oral o escrita con claridad y efectividad 
H Identifica el impacto de las soluciones de Ingeniería en el contexto global,

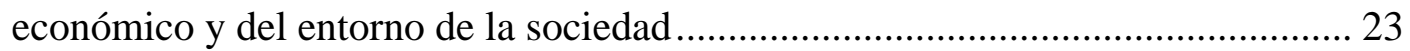

I Reconoce la necesidad de mantener sus conocimientos actualizados................. 23

J Analiza hechos del mundo contemporáneo identificando el impacto en el desempeño profesional de ingeniería

K Utiliza técnicas, herramientas y metodologías necesarias para la práctica de la ingeniería

CAC J Comprende y brinda soporte para el uso, entrega y gestión de sistemas de información dentro de un entorno de sistemas de información.............................. 25

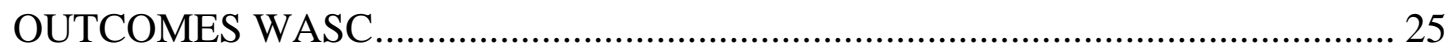

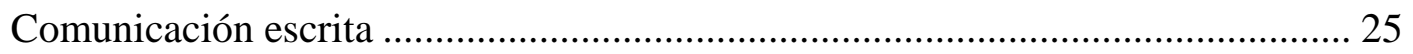

Comunicación oral.......................................................................................... 26

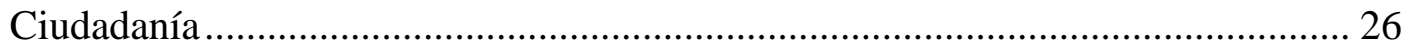

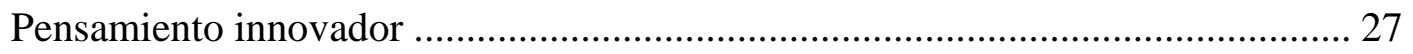

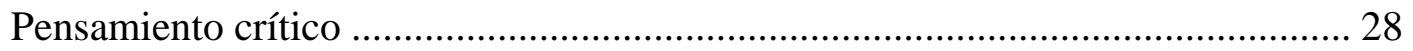

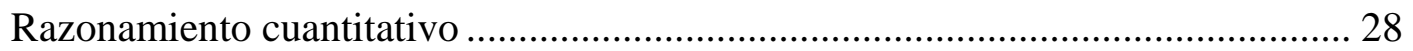

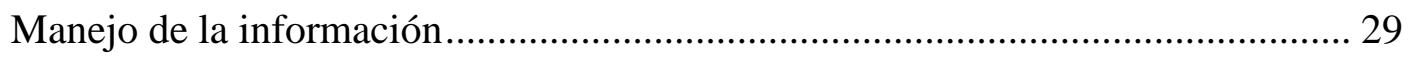

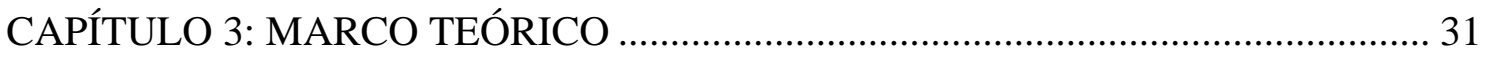

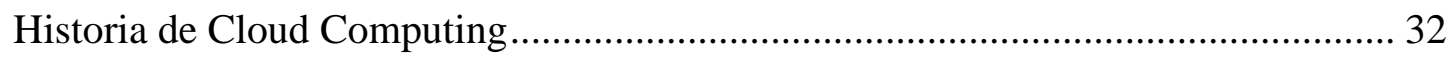

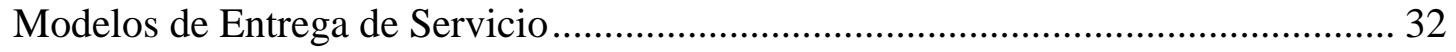

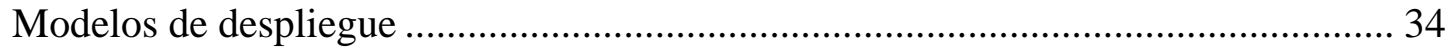

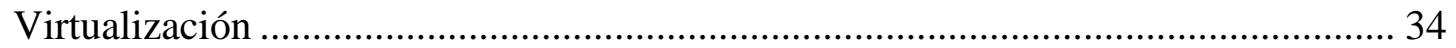

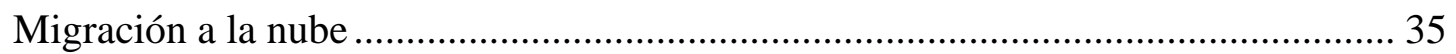

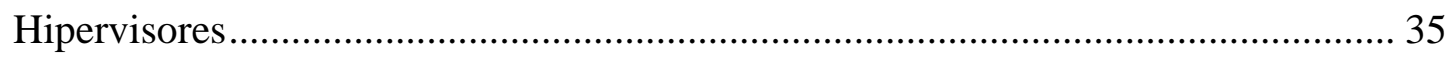

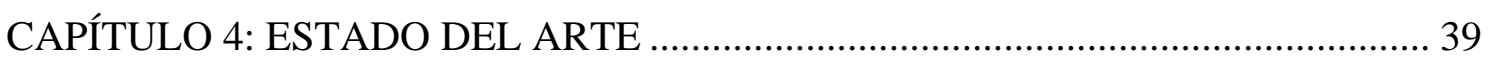

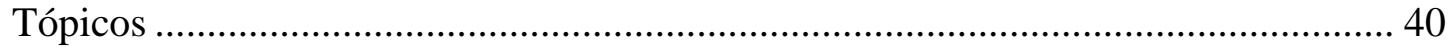

Tópico 1: Algoritmos para la migración a la nube ............................................... 40

Tópico 2: Desafíos en Cloud para la migración a la nube...................................... 40

Tópico 3: Marcos de Trabajo para la migración a la nube ..................................... 41

Tópico 4: Modelos, arquitectura y taxonomía de la migración a la nube .............. 42

Tópico 5: Optimización de migración a la nube................................................... 43

Tópico 6: Métodos para la migración a la nube ................................................... 44

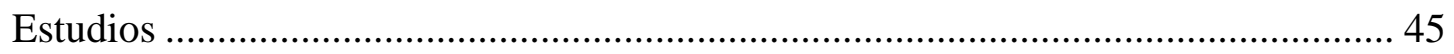


BGM-BLA: A NEW ALGORITHM FOR DYNAMIC MIGRATION OF VIRTUAL MACHINES IN CLOUD COMPUTING ....................................... 45

AUTOMATION AND ORCHESTRATION FRAMEWORK FOR LARGESCALE ENTERPRISE CLOUD MIGRATION ................................................ 46

SUPPORT VECTOR MACHINE APPROACH FOR VIRTUAL MACHINE

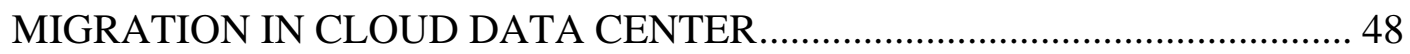
COST-MINIMIZING DYNAMIC MIGRATION OF CONTENT DISTRIBUTION SERVICES INTO HYBRID CLOUDS …....................................................... 50

CLOUDGENIUS: A HYBRID DECISION SUPPORT METHOD FOR AUTOMATING THE MIGRATION OF WEB APPLICATION CLUSTERS TO PUBLIC CLOUDS

CORRELATION BASED VIRTUAL MACHINE MIGRATION IN DYNAMIC CLOUD ENVIROMENTS.

CAPITULO 5: DESARROLLO DEL MODELO DE MIGRACIÓN A LA NUBE DE LOS SERVIDORES DE UN DATA CENTER

Referencias

Fuentes Primarias 60

Fuentes Secundarias 61

Buenas Prácticas 67

Modelo de migración a la nube de los servidores de un data center 70

Roles y responsabilidades. 72

Problemática de la empresa 73

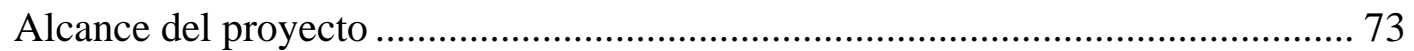

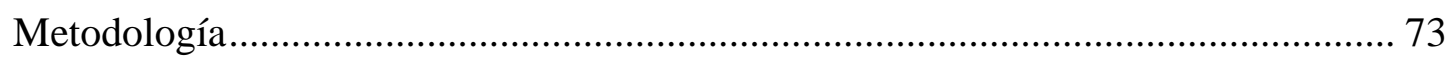

FASE I: Entendimiento ........................................................................... 76

Principios, objetivos y motivadores de negocio ............................................... 76

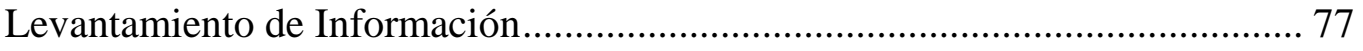

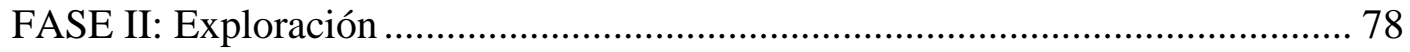

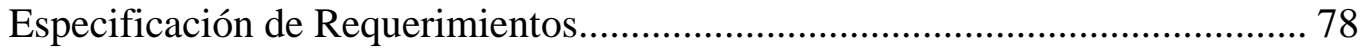

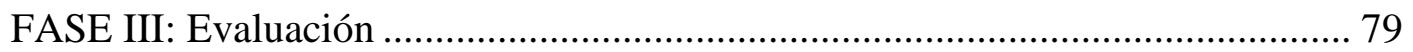

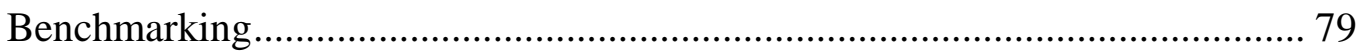

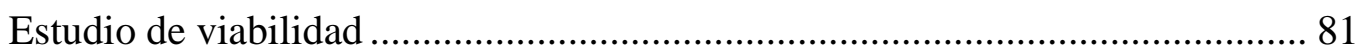

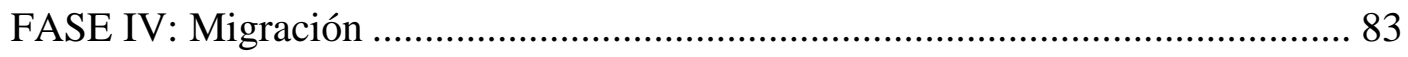

Documento de Implementación.................................................................... 83 


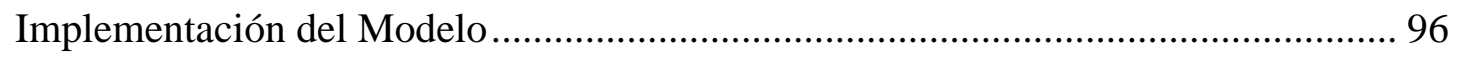

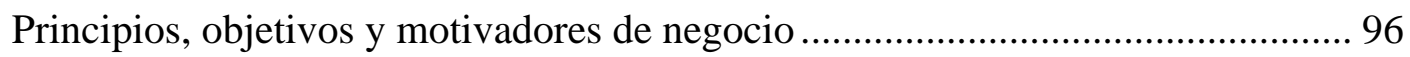

Levantamiento de Información.......................................................................... 96

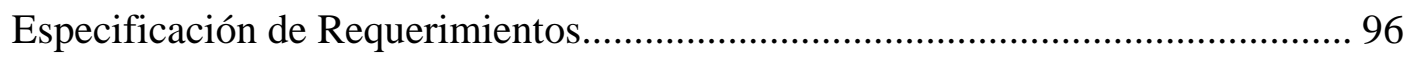

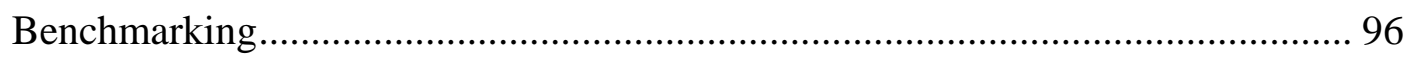

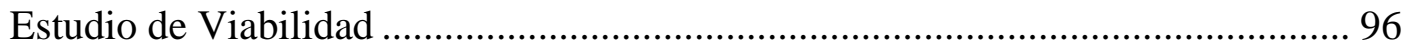

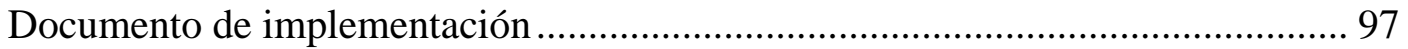

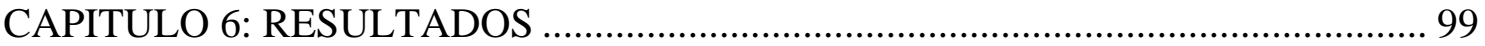

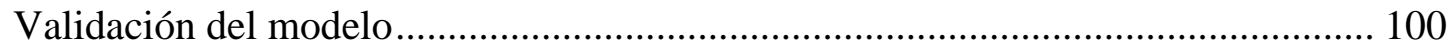

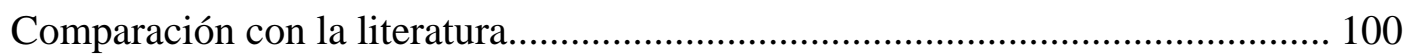

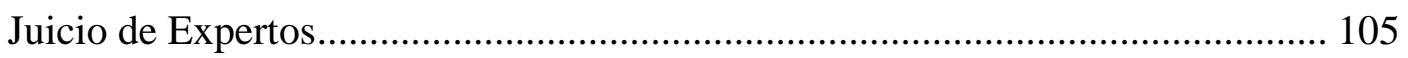

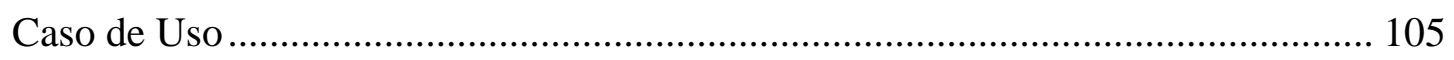

Presentación de Empresa ................................................................................... 105

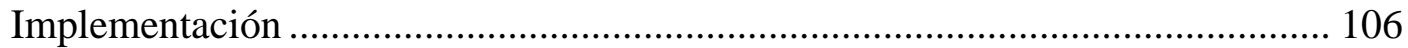

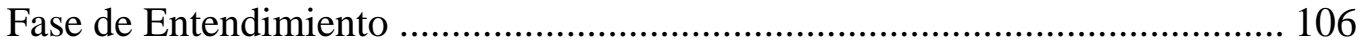

Fase de Exploración ............................................................................................ 106

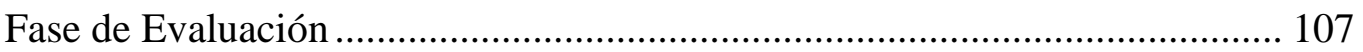

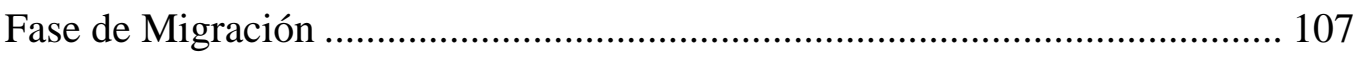

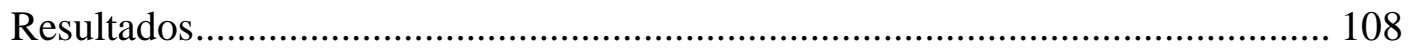

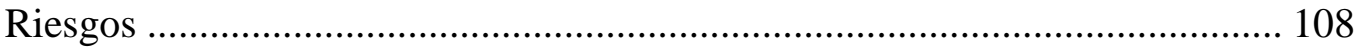

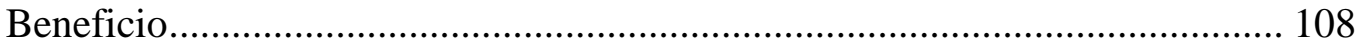

Alineamiento Estratégico ......................................................................... 111

CAPÍTULO 7: GESTIÓN DEL PROYECTO …........................................................... 114

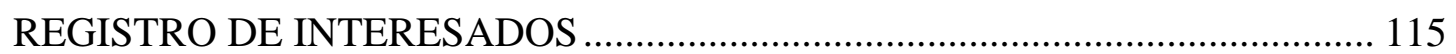

MATRIZ DE TRAZABILIDAD DE REQUERIMIENTOS ................................ 117

MATRIZ DE ASIGNACIÓN DE RESPONSABILIDADES ................................. 118

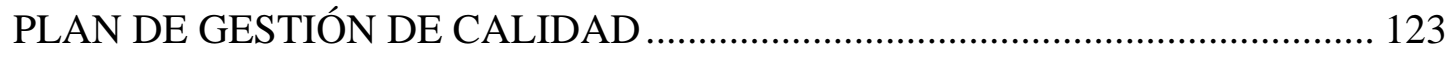

Roles y Responsabilidades ........................................................................ 123

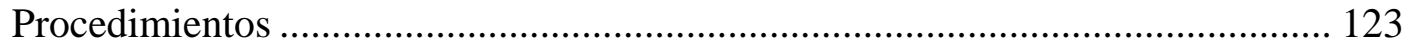

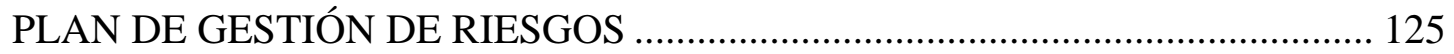

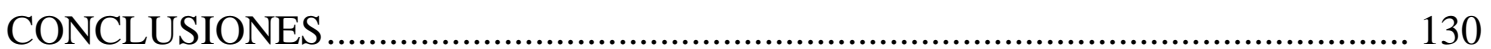

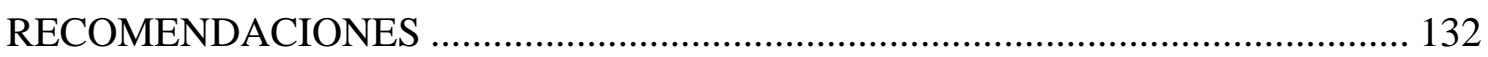

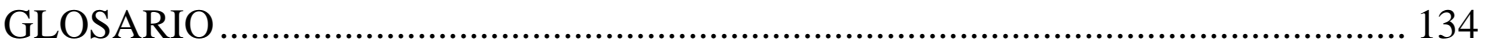

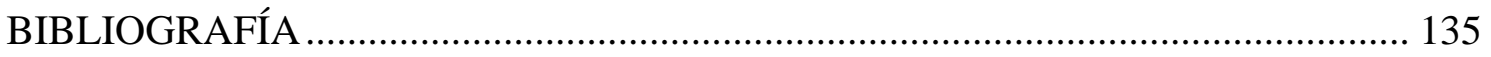


ANEXOS 


\section{Lista de Tablas}

Tabla 1: Tabla de Problema y Causas

Tabla 2: Tabla de Indicadores de Éxito

Tabla 3: Resultados del Benchmarking

Tabla 4: Comparación de factores con la literatura 104

Tabla 5: Riesgos 108

Tabla 6: Registro de Interesados

115

Tabla 7: Matriz de trazabilidad de requerimientos 117

Tabla 8: Matriz de asignación de responsabilidades 119

Tabla 9: Gestión de Riesgos 125

Tabla 10: Estrategias de Respuesta 126
15

17

80 


\section{Lista de Ilustraciones}

Ilustración 1: Método propuesto - ICCM 28

Ilustración 2: Flujo de BGM - BLA 46

Ilustración 3: CMO Framework 47

Ilustración 4: Comparación de tiempo de transferencia de datos $\quad 48$

Ilustración 5: Algoritmo de locación de las maquinas virtuales 49

Ilustración 6: Estados en migración a la nube evolutiva 52

Ilustración 7: Modelo formal de CloudGenius $\quad 52$

Ilustración 8: Complejidad de tiempo en computación paralela 53

Ilustración 9: Algoritmo SQGM 55

Ilustración 10: Algoritmo RECM 56

Ilustración 11: Hipervisor tipo 136

Ilustración 12: Hipervisor Tipo 236

Ilustración 13: Grafo del modelo de migración a la nube de los servidores de un data center 71

Ilustración 14: Modelos recopilados de la literatura 100

Ilustración 15: Factores de comparación 103

Ilustración 17: Beneficios del Proyecto 110

Ilustración 18: Retorno de Inversión 110

Ilustración 19: Alineamiento de Objetivos del Modelo

Ilustración 20: Alineamiento con los Objetivos Generales de la Empresa 112 


\section{INTRODUCCIÓN}

El proyecto consiste en la implementación de un modelo de migración a la nube, con el fin de traer mayores beneficios a la organización, esto gracias a los beneficios que brinda cloud, como es el caso de la escalabilidad, automatización, reducción de costos, entre otros. La estructura del documento se basa en 7 capítulos, los cuales son detallados a continuación.

El primer capítulo sirve de manera introductoria para conocer los objetivos del proyecto, los indicadores de éxito y la planificación inicial del mismo, esto concierne al alcance, la gestión del tiempo, la gestión de los recursos humanos, el plan de comunicaciones y los principales riesgos que el proyecto enfrenta.

El segundo capítulo describe los Student Outcomes de la carrera de Ingeniería de Sistemas de Información que el proyecto debe cumplir para estar alineado con los lineamientos del perfil del egresado de la carrera de Ingeniería de Sistemas de Información de la Universidad Peruana de Ciencias Aplicadas.

El tercer capítulo presenta al Estado del Arte, el cual presenta las revisiones de la literatura que ayudan a entender en qué contexto y bajo que problemáticas se va a desarrollar el proyecto.

El cuarto capítulo es el Marco Teórico que brinda todas las definiciones y conceptos necesarios para el correcto entendimiento del proyecto realizado.

El quinto capítulo es el de Desarrollo del Proyecto el cual presenta la metodología usada, el modelo como tal y la implementación del modelo en una organización del sector financiero.

El sexto capítulo es el de Resultados del Proyecto, en el cual se detalla y sustentan los resultados que brinda el modelo propuesto para la organización que lo implemente, en estos se incluyen los riesgos, alineamiento, entre otros.

El séptimo capítulo es el Manejo del Proyecto, el cual presenta el producto final del proyecto, y los resultados del manejo del proyecto, tales como la explicación de cómo se 
gestionó el tiempo, los recursos humanos, las comunicaciones, los riesgos y por último presenta las lecciones aprendidas por el desarrollo del proyecto. 


\section{CAPÍTULO 1: DESCRIPCIÓN DEL PROYECTO}

En este capítulo se presenta la necesidad de diseñar un modelo de migración a la nube de los servidores de un data center. Además, se plantean los objetivos generales y específicos del proyecto, así como, los indicadores de éxito y los riesgos del mismo durante su desarrollo. Por último, se presenta el alcance del proyecto

\section{Objeto de Estudio}

Actualmente, la computación en la nube ha cobrado fuerza debido a que brinda altas capacidades de escalabilidad y flexibilidad, por ese motivo diversas empresas se encuentran interesadas en adoptarla; sin embargo, muchas de estas no saben cómo migrar de manera eficiente sus servidores y aplicaciones a la nube, sin causar un impacto negativo a la organización. Por tal motivo es necesario un modelo que sirva de referencia para migrar los servidores de su data center on premise a la nube.

\section{Dominio del Problema}

El dominio del problema considerado para el presente proyecto es el siguiente:

Tabla 1: Tabla de Problema y Causas 


\begin{tabular}{|c|c|}
\hline Problema & Causas \\
\hline $\begin{array}{l}\text { El crecimiento rápido y } \\
\text { constante de los servidores en }\end{array}$ & Degradación de los servidores \\
\hline $\begin{array}{lll}\text { la industria } & \text { no } & \text { es } \\
\text { proporcional } & \text { con } & \text { la }\end{array}$ & Altos costos de mantenimiento de los servidores \\
\hline $\begin{array}{lrr}\text { escalabilidad } & \text { de } & \text { la } \\
\text { infraestructura } & \text { del } & \text { data } \\
\text { center. } & & \end{array}$ & $\begin{array}{l}\text { Altos costos de back ups } \\
\text { Alto consumo de energía }\end{array}$ \\
\hline
\end{tabular}

Fuente: Elaboración Propia

\section{Planteamiento de la Solución}

La finalidad del proyecto es diseñar un modelo de migración a la nube de los servidores de una data center, el cual servirá a organizaciones que posean un data center on premise.

Se cumplirá el propósito del proyecto de presentar un modelo de migración a la nube de los servidores de un data center, el cual será validado para corroborar que funciona de manera efectiva.

La propuesta del modelo a diseñar permitirá a las organizaciones obtener beneficios, los cuales serán:

\section{Beneficios Cualitativos:}

- Estandarización de la migración

- Gestión del cambio para la migración

- Reducción de Riesgos

- Mejora Continua

\section{Beneficios Cuantitativos:}

- Reducción de uso de recursos 


\section{Objetivos del proyecto}

\section{Objetivo General}

OG: Implementar un modelo de migración a la nube de servidores de un data center de las pymes.

\section{Objetivos Específicos}

OE1: Analizar las tecnologías y las buenas prácticas de migración a la nube.

OE2: Diseñar un modelo de migración soportado por una plataforma cloud de los servidores de un data center.

OE3: Validar el modelo de migración a la nube en base a costos, tiempo, recursos y satisfacción.

OE4: Proponer un plan de continuidad que garantice el funcionamiento del modelo en el tiempo.

\section{Indicadores de éxito}

A continuación, se muestra la tabla con los indicadores de éxito necesarios.

Tabla 2: Tabla de Indicadores de Éxito

\begin{tabular}{|l|l|l|}
\hline Código del Indicador & Indicadores de Éxito & Objetivos Específicos \\
\hline IE1 & $\begin{array}{l}\text { Acta de aprobación del documento } \\
\text { "Buenas prácticas en migración a la } \\
\text { nube" }\end{array}$ & OE1 \\
\hline IE2 & $\begin{array}{l}\text { Acta de aprobación del documento } \\
\text { "Diseño del modelo de migración a } \\
\text { la nube de servidores de un data } \\
\text { center" }\end{array}$ & OE2 \\
\hline
\end{tabular}




\begin{tabular}{|l|l|l|}
\hline IE3 & $\begin{array}{l}\text { Acta de aprobación de la } \\
\text { "Validación del modelo de } \\
\text { migración a la nube". }\end{array}$ & OE3 \\
\hline IE4 & $\begin{array}{l}\text { Acta de aprobación del "Plan de } \\
\text { Continuidad". }\end{array}$ & OE4 \\
\hline
\end{tabular}

Fuente: Elaboración Propia

\title{
Planificación del Alcance
}

\begin{abstract}
Alcance
El alcance del proyecto a realizar abarcará los documentos de gestión correspondientes al "Project Management Body of Knowledge" o PMBOK; además también se incluirá lo siguiente:
\end{abstract}

- Buenas Prácticas de Migración a la nube

- El levantamiento de información de los servidores del data center

- El Diseño del modelo de migración a la nube de los servidores de un data center.

- Migración según el criterio de los stakeholders (asociados a la validación) 


\section{CAPITULO 2: LOGROS DEL PROYECTO}

En el siguiente capítulo se contempla el cumplimiento de los Student Outcomes de la carrera de Ingeniería de Sistemas de Información, y cómo estos permiten que el proyecto se realice acorde a los objetivos y al perfil del ingeniero de sistemas de información de la UPC. 


\section{OUTCOMES ABET}

\section{A Aplica conocimientos de matemáticas, ciencias, computación e ingeniería}

Aplica conocimientos de matemáticas, ciencias, computación e ingeniería (ABET, 2014), este Outcome refleja los conocimientos adquiridos por el estudiante en matemáticas, ciencias, computación e ingeniería. Para el proyecto, este student outcome se encuentra en el diseño del modelo y el método desarrollados.

En las evidencias y/o entregables se cuenta con los documentos de Current IT Enviroment, Benchmarking y Estudio de viabilidad donde se desarrolla parte del modelo que permite completar este outcome.

Para mayor información revisar los siguientes documentos:

- Current IT Enviroment

- Benchmarking

- Estudio de viabilidad

\section{B Diseña y conduce experimentos en base al análisis e interpretación de datos}

Diseña y conduce experimentos en base al análisis e interpretación de datos (ABET, 2014), este Outcome refleja al análisis que se realizó de la literatura para poder desarrollar el modelo de migración, por tal motivo el student outcome se puede encontrar durante el capítulo 5 de la memoria.

En las evidencias y/o entregables se cuenta con el capítulo 5 de la memoria, desarrollo del proyecto.

Para mayor información revisar los siguientes documentos:

- Memoria del proyecto

C Diseña sistemas, componentes o procesos para encontrar soluciones en la atención de necesidades teniendo en cuenta restricciones 


\section{económicas, sociales, políticas, éticas de salud y seguridad y otras propias del entorno empresarial}

Diseña sistemas, componentes o procesos para encontrar soluciones en la atención de necesidades teniendo en cuenta restricciones económicas, sociales, políticas, éticas de salud y seguridad y otras propias del entorno empresarial (ABET, 2014), este Outcome refleja el análisis que realizamos de la literatura, para diseñar el modelo de migración a la nube de los servidores de un data center. Para este student outcome, realizamos los componentes del modelo, que permiten migrar su infraestructura física a cloud, permitiendo ahorrar costos y aprovechar las ventajas que brinda cloud como escalamiento y automatización, entre otros.

En las evidencias y/o entregables se encuentra el documento del modelo de migración y el documento de Implementación, en el cual se detalla el proceso de migración y las conclusiones, que indican los resultados, los beneficios obtenidos para la organización, entre otros.

- Documento del modelo.

- Documento de implementación.

\section{Participa en equipos multidisciplinarios desarrollando sus tareas con profesionales de diferentes especialidades o dominios de aplicación}

Participa en equipos multidisciplinarios desarrollando sus tareas con profesionales de diferentes especialidades o dominios de aplicación (ABET, 2014), este Outcome refleja el trabajo en equipo que fue necesario para alcanzar el fin del proyecto, en el cual participamos además de ambos jefes de proyectos, los asesores y gerentes de la empresa virtual DATACENTER. Por otro lado, se tuvieron reuniones con el PMO y especialistas en electrónica de la empresa con la cual se validó el modelo para poder entender el funcionamiento de la empresa y verificar como se realizó el avance del proyecto.

En las evidencias y/o entregables tenemos las actas de reuniones realizadas con los acuerdos que se tomaron en cada una de ella. 


\section{E Identifica, formula y resuelve problemas de Ingeniería}

Identifica, formula y resuelve problemas de Ingeniería (ABET, 2014), este Outcome hace referencia al problema y como fue solucionado con el modelo propuesto en base a un estudio.

En las evidencias y/o entregables tenemos al Benchmarking y al mismo modelo desarrollado.

Para mayor información revisar los siguientes documentos:

- Benchmarking

- Documento del modelo de migración a la nube de los servidores de un data center

\section{F Propone soluciones a problemas de Ingeniería con responsabilidad profesional y ética}

Propone soluciones a problemas de Ingeniería con responsabilidad profesional y ética (ABET, 2014), este Outcome hace referencia a la gestión continua que se le dio al proyecto y al modelo propuesto.

En las evidencias y/o entregables tenemos al Project Charter y a todos los documentos de gestión utilizados del PMBOK.

Para mayor información revisar los siguientes documentos:

- Project Charter

\section{G Comunica ideas o resultados de manera oral o escrita con claridad y efectividad}

Comunica ideas o resultados de manera oral o escrita con claridad y efectividad (ABET, 2014), este Outcome hace referencia a todas las comunicaciones que se realizaron durante el proyecto. Para este punto, se tuvieron diversas reuniones tanto con el Profesor Gerente como con el Profesor Asesor, además de presentar sustentaciones parciales y finales como hitos importantes dentro del proyecto y el paper y la memoria del proyecto. 
En las evidencias y/o entregables contamos con actas de reunión firmada por los asistentes en los cuales se detallan los acuerdos que se tomaron. Además del paper y memoria del proyecto.

\section{H Identifica el impacto de las soluciones de Ingeniería en el contexto global, económico y del entorno de la sociedad}

Identifica el impacto de las soluciones de Ingeniería en el contexto global, económico y del entorno de la sociedad (ABET, 2014), este Outcome hace referencia a la propuesta de valor que le brindamos a la empresa y como la diferenciamos de cualquier otro producto que se pueda encontrar en el mercado. Para esto se realizó un estudio sobre la empresa y las diversas herramientas que se pueden utilizar.

En las evidencias y/o entregables contamos con el entendimiento del negocio, benchmarking y la evaluación de viabilidad.

Para mayor información revisar los siguientes documentos:

- Principios, objetivos y motivadores de negocio

- Levantamiento de Información

- Benchmarking

- Estudio de Viabilidad

\section{Reconoce la necesidad de mantener sus conocimientos actualizados}

Reconoce la necesidad de mantener sus conocimientos actualizados (ABET, 2014), este Outcome hace referencia a la necesidad de que el modelo propuesto se encuentre actualizado de una manera competitiva en el mercado. Para lograr esto, el método usado es el constante estudio de las tecnologías a utilizar por medio de diversos papers de investigación publicados por journals especializados en los temas tratados.

En las evidencias y/o entregables contamos con el Modelo Tecnológico y el plan de continuidad en el cual se trazará la constante actualización y el futuro del modelo propuesto.

Para mayor información revisar los siguientes documentos: 
- Modelo de migración a nube de los servidores de un data center

- Plan de continuidad

\section{J Analiza hechos del mundo contemporáneo identificando el impacto en el desempeño profesional de ingeniería}

Analiza hechos del mundo contemporáneo identificando el impacto en el desempeño profesional de ingeniería (ABET, 2014), este Outcome hace referencia a cómo la actualidad y los hechos de importancia impactan al proyecto que hemos realizado. Para esto se han realizado diversas investigaciones y comparaciones en el benchmarking, tratando de ver que impactos podrían tener los hechos importantes en el proyecto y en la continuidad del modelo.

En las evidencias y/o entregables contamos con el Benchmarking el cual es el documento principal donde plasmamos toda la investigación con respecto a la tecnología.

Para mayor información revisar los siguientes documentos:

- Benchmarking

\section{K Utiliza técnicas, herramientas y metodologías necesarias para la práctica de la ingeniería}

Utiliza técnicas, herramientas y metodologías necesarias para la práctica de la ingeniería (ABET, 2014), este Outcome hace referencia al manejo que le dimos al proyecto de principio a fin y como se realizó éste. Para ello, utilizamos la metodología PMBOK para la gestión continua del proyecto, además diseñamos un método al cual llamamos ICMM (Infrastructure Cloud Migration Method), el cual está basado en CCRA v4, ADM TOGAF y la investigación de papers.

En las evidencias y/o entregables contamos con todos los entregables y artefactos del PMBOK y el documento del modelo propuesto. 


\section{CAC J Comprende y brinda soporte para el uso, entrega y gestión de}

sistemas de información dentro de un entorno de sistemas de información

Comprende y brinda soporte para el uso, entrega y gestión de sistemas de información dentro de un entorno de sistemas de información (ABET, 2014), este Outcome hace referencia a las acciones de soporte que garanticen la continuidad del proyecto. Para ello se realizó un plan en el cual se definen roles, responsabilidades y acciones a realizar en caso de incidencias.

En las evidencias y/o entregables encontramos el plan de soporte en el cual se define lo antes mencionado.

Para mayor información revisar los siguientes documentos:

- Plan de soporte.

\section{OUTCOMES WASC}

\section{Comunicación escrita}

Se define como la capacidad para construir mensajes con contenido relevante, argumentos sólidos y claramente conectados adecuados a diversos propósitos y audiencia.

Esta capacidad se evidencia en la redacción de la memoria, entregable e informes de los objetivos realizados. En este proyecto fueron:

- Memoria Final del Proyecto: MMNSDC - Modelo de Migración a la nube de los servidores de un data center.

- Informe de Objetivo 1: Análisis de Buenas Prácticas para la migración.

- Informe de Objetivo 2: Descripción del Modelo de Migración a la nube de los servidores de un data center.

- Entregables del Modelo:

- Principios, Objetivos y Motivadores de Negocio

- Levantamiento de Información 
- Especificación de Requerimientos

- Benchmarking

- Estudio de Viabilidad

- Documento de Implementación

- Paper del Proyecto

\section{Comunicación oral}

Se enfoca en la capacidad para transmitir oralmente mensajes de manera eficaz dirigidos a diversas audiencias, usando diferentes herramientas que faciliten su comprensión y el logro del propósito.

Esta capacidad se puede evidenciar en las reuniones que se ha tenido con los profesores clientes y gerentes. Para la guía, acompañamiento y revisión del proyecto También para el caso de uso, se usó la comunicación oral para transmitirle la idea del proyecto y sus beneficios a la empresa Globokas. Se tuvo reuniones con los encargados de los servidores y con el Gerente de PMO de la empresa.

También en las exposiciones con los profesores, clientes, gerentes y comités. En las presentaciones parciales y finales correspondientes.

\section{Ciudadanía}

Dicha capacidad es para valorar la convivencia humana en sociedades plurales, reflexionando acerca de las dimensiones morales de las propias acciones y decisiones, asumiendo la responsabilidad por las consecuencias en el marco del respeto de los derechos y deberes ciudadanos.

La ciudadanía se evidencia cuando se ha analizado el impacto del proyecto propuesto. Puesto que se ha realizado una viabilidad económica para analizar el impacto financiero y retorno que les puede afectar a la empresa Globokas. Ya que para realizar cualquier proyecto se tiene que verificar si le es rentable o no. También al explicarle los beneficios que ellos tendrían con el proyecto y el cómo los impactaría al llevar sus servidores a la nube. También el respeto que se ha tenido con el profesor cliente, gerente y comité. Como 
último, se evalúan las políticas que tiene la empresa con el proyecto para que estén alineados.

Pensando en la continuidad que pueda tener para la ciudadanía se plantea futuros proyectos que a futuro les pueda servir:

- Modelo de migración a la nube de microservicios (contenedores) de un data center.

- Modelo de integración para PYMES de la transformación digital basada en cloud

- Modelo de calidad de servicios cloud para PYMES peruanas.

\section{Pensamiento innovador}

Esta capacidad sirve para detectar necesidades y oportunidades para generar proyectos o propuestas innovadoras, viables y rentables. Planifica y toma decisiones eficientes orientadas al objetivo del proyecto.

Cuando se planteó el problema, se pensó en una forma en la cual sea sencilla y eficiente la migración a la nube de los servidores; por lo que se creó el método ICMM: Infraestructura Cloud Migration Method. Dentro de este se definieron fases para que la sencillez y eficiencia se logre de forma ordenada. También se definieron roles para el proyecto para que no repitan tareas y se tenga un doble recursos, se propone que se realice la mejora continua en el proyecto, se analizan cada activo de TI a migrar y se evalúa su viabilidad operativa, financiera y técnica. Pensando en el usuario y en su operatividad, se definió que la migración sea en horarios fuera de oficina. 


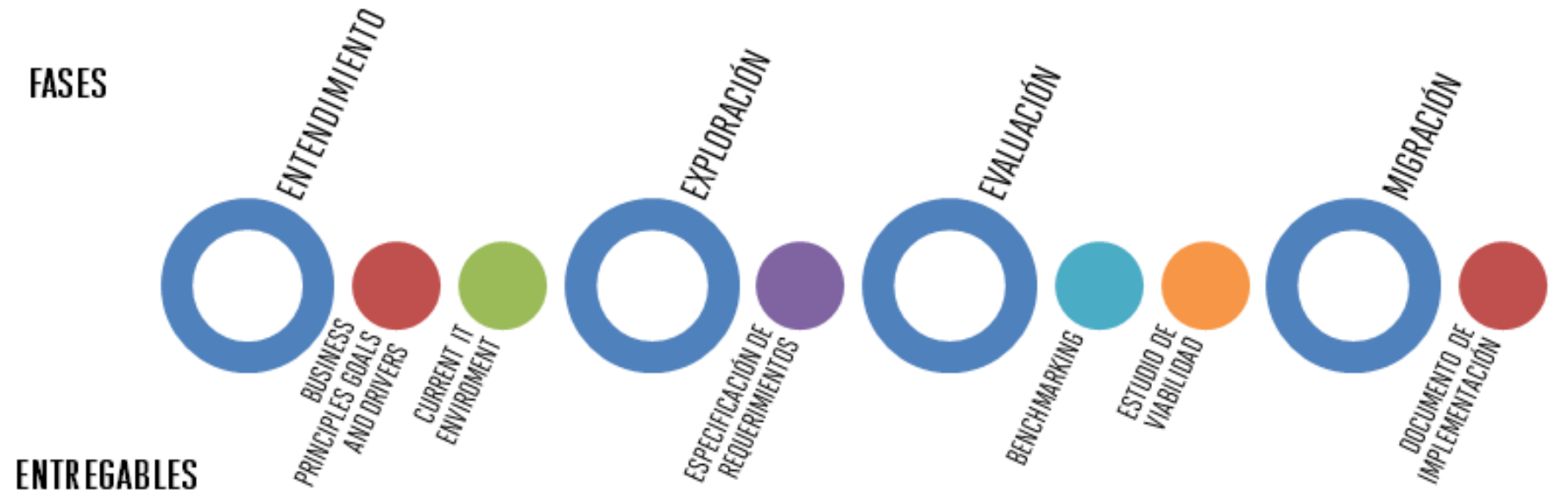

Ilustración 1: Método propuesto - ICCM

Fuente: Elaboración Propia

\section{Pensamiento crítico}

Se define como la capacidad para explorar de manera exhaustiva problemas, ideas o eventos para formular conclusiones u opiniones sólidamente justificadas.

Se evalúo el problema cuando se planteó el proyecto: La falta de escalabilidad del data center en la industria. Por lo que se pensó en una solución en base a lo analizado en las buenas prácticas, previamente investigadas. Ya que se evaluó varios escenarios posibles y se llegó a la conclusión que para el modelo se necesita una viabilidad técnica para ver que sea compatible con la plataforma escogida en el Benchmarking. Teniendo ya todo listo solo para la parte operativa, se tiene una base sólida como sustento del modelo.

\section{Razonamiento cuantitativo}

Es la capacidad para interpretar, representar, comunicar y utilizar información cuantitativa diversa en situaciones de contexto real. Incluye calcular, razonar, emitir juicios y tomar decisiones con base en esta información cuantitativa.

Para realizar el retorno de inversión del proyecto, se realizó la estimación, los beneficios cuantitativos y un flujo de caja para evaluar en cuantos meses la empresa iba a recuperar 
su inversión. Para ello se utilizó finanzas y estadística. Eso se puede evidenciar más a detalle en el capítulo 6 de la memoria.

También cuando se analizó los resultados del proyecto. Se tuvo que realizar la comparación del método con los objetivos de la empresa en el proyecto y presentar porcentajes. Para realizarlo de uso matemáticas en la cuantificación. Para más detalle se puede revisar el capítulo 6 de la memoria.

\section{Manejo de la información}

Se enfoca en la capacidad de identificar la información necesaria, así como de buscarla, seleccionarla, evaluarla y usarla éticamente, con la finalidad de resolver un problema.

Para cumplir con esta capacidad se realizó el Objetivo 1: Analizar las buenas prácticas de la migración a la nube. Con el cual se recopilo información de la literatura (artículos científicos) y proveedores de cloud: IBM Bluemix, RedHat, Amazon Web Services, Microsoft Azure. Esta información se clasificó en 3 partes: Antes de la migración, Durante la migración y Después de la migración.

También se usó como fuente CCRA v4 el cual brinda una metodología Cloud. Con toda esta información recopilada se pudo diseñar el modelo y sus componentes. 


\section{CAPÍTULO 3: MARCO TEÓRICO}

El presente capítulo presenta la definición de todos los términos y conceptos esenciales en el ámbito de migración a la nube para permitir que el lector se familiarice con el tema del proyecto. Dentro de los conceptos a desarrollar, se encuentran los conceptos básicos y complementarios. 


\section{Historia de Cloud Computing}

Cloud Computing es una evolución natural de la adopción generalizada de la virtualización, la arquitectura orientada a servicios y utilidad de cómputo. La idea es que los usuarios finales no requieran conocimientos ni control sobre la infraestructura.

El concepto básico de Cloud Computing se le atribuye a John McCarthy, quien el 1961 fue el primer hombre en proponer que la tecnología de tiempo compartido de las computadoras podría conducir a un futuro donde el poder de cómputo o las aplicaciones podrían venderse como servicio.

Con el tiempo diversas empresas como salesforce y Amazon brindaban ya servicios del tipo, en el año 2006, apareció Google Docs, que realmente trajo el Cloud Computing a la vanguardia de la consciencia del público.

Fue entonces cuando empresa como IBM, Microsoft, Apple, entre otras entraron a brindar estos modelos de servicios. Actualmente se sabe que algunos de estos proveedores, ya sea por fallas en la infraestructura o vulnerabilidades, que no son $100 \%$ confiables. ${ }^{1}$

\section{Modelos de Entrega de Servicio}

- Infrastructure as a Service (IaaS): Es uno de los tres modelos fundamentales en el campo de Cloud Computing. Al igual que todos los servicios cloud, IaaS proporciona acceso a recursos informáticos situados en un entorno virtualizado. Los recursos informáticos ofrecidos consisten en hardware virtualizado. Abarca aspectos como el espacio de servidores virtuales, conexiones de red, ancho de banda, conexiones IP y balanceadores de carga.

Las ventajas de una implementación basada en el modelo de IaaS:

- Escalabilidad:

- No se necesita invertir en hardware

- Independencia de la localización

- Seguridad física en los centros de datos ${ }^{2}$

\footnotetext{
${ }^{1}$ Cfr. Eric Tecayehuatl (2012)

${ }^{2}$ Cfr. Interoute (2014)
} 
- Platform as a Service (PaaS): es un entorno de desarrollo e implementación completo en la nube, con recursos que permiten entregar todo, desde aplicaciones sencillas basadas en la nube hasta aplicaciones empresariales sofisticadas habilitadas para la nube. Uno le compra los recursos que necesita a un proveedor de servicios en la nube, a los que accede a través de una conexión segura a Internet, pero solo paga por el uso que hace de ellos. Al igual que IaaS, PaaS incluye infraestructura (servidores, almacenamiento y redes), pero también incluye middleware, herramientas de desarrollo, servicios de inteligencia empresarial, sistemas de administración de bases de datos, etc. Está diseñado para sustentar el ciclo de vida completo de las aplicaciones web: compilación, pruebas, implementación, administración y actualización. Permite evitar el gasto y la complejidad que suponen la compra y la administración de licencias de software, la infraestructura de aplicaciones y el middleware subyacentes, las herramientas de desarrollo y otros recursos. Usted administra las aplicaciones y los servicios que desarrolla y, normalmente, el proveedor de servicios en la nube administra todo lo demás. ${ }^{3}$

- Software as a Service (SaaS): es un modelo de distribución de software donde el software y los datos se alojan en servidores del proveedor y se accede con un navegador web a través de Internet. El Software Saas tiene una característica que hace que el modelo sea especialmente eficiente: es multitenancy. Esta es la propiedad que permite ofrecer la misma aplicación a muchos usuarios y así distribuir el coste de la infraestructura y del mantenimiento entre todos. Es decir una aplicación que permite con una sola instancia y una sola base de datos dar servicio a todos los clientes. ${ }^{4}$

\footnotetext{
${ }^{3}$ Cfr. Microsoft Azure (2015)

${ }^{4}$ Cfr. Portal Saas (2014)
} 


\section{Modelos de despliegue}

- Nube Pública: La infraestructura de la nube está disponible al público en general (o un subconjunto en función de los criterios de venta del Proveedor). La infraestructura pertenece a la organización que vende sus servicios de Cloud Computing.

- Nube Privada: La infraestructura de la nube pertenece a una única organización, quien la ofrece como servicio a sus propios departamentos. Puede ser gestionada por la organización o por una tercera parte, y puede estar en los locales de la organización o fuera de ellos.

- Nube Comunitaria: La infraestructura de la nube es compartida por varias organizaciones y da soporte a una comunidad específica que comparte las mismas preocupaciones (p.e. misión, seguridad, requisitos, consideraciones de normativa legal, etc.).

- Nube Híbrida: La infraestructura de la nube está compuesta por dos o más tipos de nubes (privada, pública o comunitaria) que mantienen su propia identidad pero que son unidas por una tecnología propietaria o estándar para permitir la portabilidad de datos y aplicaciones. ${ }^{5}$

\section{Virtualización}

La virtualización de un sistema operativo consiste en usar software para permitir que un elemento de hardware gestione múltiples imágenes de sistemas operativos al mismo tiempo. Esta tecnología empezó a utilizarse con las computadoras de gran tamaño (mainframes) hace ya décadas, y permitió a los administradores ahorrar en recursos de procesamiento. ${ }^{6}$

Ventajas:

- Desarrolle la continuidad del negocio con soluciones de recuperación ante desastres mejoradas y proporciona alta disponibilidad en todo el centro de datos.

\footnotetext{
${ }^{5}$ Cfr. Realcloud (2012)

${ }^{6}$ Cfr. Margaret Rouse (2014)
} 
- Virtualizar las aplicaciones empresariales, tales como Oracle, Exchange, SQL Server, Sharepoint o SAP, y proporcione los máximos niveles de servicio y un rendimiento superior.

- Favorecerá la comercialización de sus productos y/o servicios.

- Optimiza las inversiones y simplifica la gestión de TI.

- Los servidores estarán preparados para responder a cambios en las cargas de trabajo.

\section{Migración a la nube}

Este modelo tiene mucho sentido como método para consolidar gastos y recursos de informática, automatizar tareas e introducir nuevas tecnologías como la virtualización. A menudo, este modelo provee un puente entre sistemas heredados y nuevas maneras de pensar sobre la tecnología.

Muchas organizaciones de informática se están mudando a la computación en la nube creyendo que se trata únicamente de virtualizar. En muchos centros de datos, una plataforma de virtualización es básica para la oferta de servicios, pero las nubes no se definen solamente por la tecnología. En vez de eso, involucran a gente, procesos, centralización y control. Mudarse a la nube promete consolidar servicios duplicados dentro de una organización y automatizar tareas rutinarias, que se hacen sin pensar, para que los empleados estén libres para trabajar sobre problemas difíciles.

\section{Hipervisores}

Los hipervisores son la parte principal de una máquina virtual que se encarga de manejar los recursos del sistema principal exportándolos a la máquina virtual.

Los hipervisores pueden clasificarse en dos tipos:

- Hipervisor tipo 1: También denominado nativo o bare metal, es software que se ejecuta directamente sobre el hardware, para ofrecer la funcionalidad descrita. 


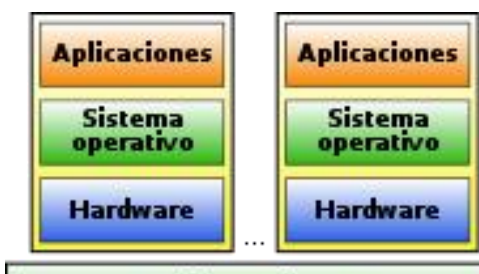

Hipervis or

Hardware real

Ilustración 2: Hipervisor tipo

1

Algunos de los hipervisores tipo 1 más conocidos son los siguientes: VMware ESXi, Xen, Citrix XenServer, Microsoft Hyper-V Server, Oracle VM.

- Hipervisor tipo 2: También denominado hosted, es software que se ejecuta sobre un sistema operativo para ofrecer la funcionalidad descrita.

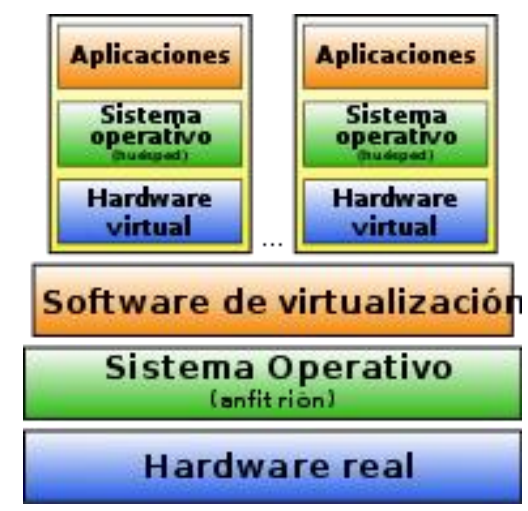

Ilustración 3: Hipervisor

Tipo 2 
Algunos de los hipervisores tipo 2 más utilizados son los siguientes: Oracle: VirtualBox, VirtualBox OSE, VMware: Workstation, Server, Player, QEMU, Microsoft: Virtual PC. 


\section{CAPÍTULO 4: ESTADO DEL ARTE}

Este capítulo expone los trabajos científicos realizados previamente referente a la migración a la nube, tanto como desafíos, modelos o marcos de trabajo. 


\section{Tópicos}

\section{Tópico 1: Algoritmos para la migración a la nube}

De acuerdo a los autores, se establece un algoritmo para un modelo de optimización con un triple objetivo para la migración dinámica de máquinas virtuales. Por ejemplo, solucionar los problemas del consumo de energía, la comunicación entre máquinas virtuales y el costo de la migración ${ }^{7}$. Asimismo, se propone un algoritmo de control dinámico para colocar la forma óptima los contenidos y enviar a las solicitudes en una nube híbrida, basada en técnicas de Lyapunov. Lo que minimiza el costo operacional a lo largo del tiempo ${ }^{8}$. También proponen otro algoritmo para la asignación de máquinas virtuales entre máquinas físicas, que se basa en la máquina de vector de soporte, para minimizar el número de migración de máquinas virtuales y el uso de recursos en las máquinas físicas ${ }^{9}$.

\section{Tópico 2: Desafíos en Cloud para la migración a la nube}

Entre los desafíos de cloud que se presentan en los artículos previamente investigados, se encuentra la evaluación de los factores que afectan la adopción en Cloud Computing en relación al desarrollo de la economía de las PYMEs ${ }^{10}$. Otro de los desafíos con lo que nos podemos encontrar son con los proveedores de la plataforma. Se proponen varias estrategias de cómo mitigar estos riesgos con los terceros. Dichas estrategias se relacionan con los contratos, la selección de proveedores que soportan protocolos estandarizados con respecto a la estructura de datos y APIs. De esta manera se van desarrollando las dependencias entre las soluciones basadas en la nube ${ }^{11}$. Entre otros desafíos se encuentran los que se presentan para migrar los sistemas heredados hacia la plataforma de la nube, los cuales mediante una herramienta se organizan y analizan para que se tenga alternativas de migración ${ }^{12}$.

\footnotetext{
${ }^{7}$ Cfr. Fei Tao, Chen Li, T Warren Liao y YuanjunLaili (2015)

${ }^{8}$ Cfr. Xuanjia Qiu, Hongxing Li, Chuan Wu, Zongpeng Li and Francis C.M. Lau (2017)

${ }^{9}$ Cfr. Fan-Hsun Tseng, Xiaojiao Chen, Li-Der Chou, Han-Chieh Chao, Shiping Chen (2014)

${ }^{10}$ Cfr. Abdullah, I, Hassan, Z. (2015)

${ }^{11}$ Cfr. Justice Opara-Martins, Reza Sahandi and Feng Tian (2016)

12 Cfr. Mahdi Fahmideh Gholami, Farhad Daneshgar, Ghassan Beydoun, Fethi Rabhi (2017)
} 


\section{Tópico 3: Marcos de Trabajo para la migración a la nube}

Los autores consideran que una de las principales razones por las cuales las organizaciones no adoptan cloud, es por la falta de consciencia, por lo tanto, proponen un marco de trabajo confiable para la migración a la nube, que consta de 7 fases, las cuales son: Iniciación, adopción, toma de decisión y selección, migración, adaptación y control, mantenimiento y optimización, se ha realizado validación del marco de trabajo propuesto. Adicionalmente también proveen un modelo de madurez de migración a cloud (CIM3) ${ }^{13}$.

En otro trabajo, se propone CMO (Cloud Migration Orchestator), el cual es un framework que para la automatización y coordinación de a gran escala de la migración a la nube, está basado en BPM (Business Process Management), y es conformado por las siguientes fases: discovery engine, Analytics engine, configuration engine y migration engine. La validación del mismo ha demostrado su efectividad para migrar servidores de diferentes sistemas operativos eficientemente, aumentando la velocidad de migración considerablemente ${ }^{14}$.

Un grupo de investigadores, realizaron una evaluación de marcos de trabajo existentes, en la cual explican las propuestas y muestran las diferencias y estadísticas de cada una, cabe destacar que los documentos evaluados están enfocados en migración a la nube de sistemas legacies ${ }^{15}$.

Un grupo de autores, propuso CloudGenius, que es un método de soporte de decisiones híbrido para automatizar la migración de clústeres de aplicaciones web a nubes públicas, una característica de este framework es el modelo del proceso de migración evolutivo a cloud, el cual integra los enfoques de migración existentes y métodos para soportar decisiones basados en múltiples criterios, para seleccionar imágenes de VM y servicios de cómputo para diferentes componentes ${ }^{16}$.

En otro estudio proponen un novedoso framework conceptual de para migración de live virtual machines, el cual está basado en un enfoque de regresión lineal, el cual es útil cuando se requiere predicciones, otro de sus aspectos claves es "smart pre copy live

\footnotetext{
13 Cfr. Hamid Reza Bazi, Alireza Hasanzadeh, Ali Moeini (2017)

${ }^{14}$ Cfr. J. Hwang, K. Bai, M. Tacci, M. Vukovic, N. Anerousis (2016)

15 Cfr. Mahdi Fahmideh Gholami, Farhad Daneshgar, Graham Low, Ghassan Beydoun (2016)

${ }^{16}$ Cfr. Michael Menzel, Rajiv Ranjan, Lizhe Wang, Samee U. Khan, Jinjun Chen (2014)
} 
migration", el cual asegura la alta disponibilidad, y disminuye el downtime luego de cada iteración usando la regresión lineal ${ }^{17}$.

También, se encontró un trabajo, en el cual realizan una investigación sobre la migración a la nube, sobre la cual concluyen que existe una falta de validación de investigaciones y opinión de los papers. Por otro lado, las investigaciones con enfoque de migración ignoran factores del contexto y situaciones reales ${ }^{18}$.

Además, los autores proponen un framework de optimización para la migración a la nube, lo que buscan es mejorar la confiabilidad en los sistemas y tolerancia a fallos, las estrategias basadas en características de cloud también pueden mejorar la confiabilidad, debido a su tolerancia a fallos, las características deseadas son: recovery block, $\mathrm{N}$-version programming, paralelismo y reinicio de $\mathrm{VM}^{19}$.

\section{Tópico 4: Modelos, arquitectura y taxonomía de la migración a la nube}

Los autores desarrollan un enfoque integrado para abordar la selección de máquinas virtuales a migrar, los problemas de selección de servidores. Con el fin de identificar el momento preciso para la migración. Para ello proponen un modelo de migración basado en SLA, con el cual reduce el número de migraciones significativamente ${ }^{20}$. Otra propuesta realizada por los autores es un modelo conceptual que da lugar a una base de recursos de soluciones existentes para la migración a la nube ${ }^{21}$. Asimismo, se está proponiendo una taxonomía temática detallada que propone la categorización de migración de VM. Los que incluyen aspectos críticos y características relacionadas a los esquemas de migración de VM. Estos son inspeccionados a través de una investigación cualitativa detallada ${ }^{22}$.

\footnotetext{
${ }^{17}$ Cfr. Opeyemi O., Shuo Chen, Zheng Yan, Rongxing Lu, Kim-Kwang Raymond Choo, Mqhele D. (2017)

18 Cfr. Pooyan Jamshidi, Aakash Ahmad, and Claus Pahl (2014)

${ }^{19}$ Cfr. Weiwei Qiu, Zibin Zheng, Xinyu Wang, Xiaohu Yang, Michael R. Lyu (2014)

${ }^{20}$ Cfr. Liu, Shaoping Zheng, Hongfang Yu, Vishal Anand, Du Xu (2015)

${ }^{21}$ Cfr. Rai Rashmi, Sahoo Gadadhar, Mehfuz Shabana (2015)

${ }^{22}$ Cfr. Ahmad Raja, Gani Abdullah, Ab. Hamid Siti, Shiraz Muhammad, Xia Feng, Madani Sajjad (2015)
} 


\section{Tópico 5: Optimización de migración a la nube}

Los autores proponen distintos enfoques de optimización de la migración a la nube, uno de ellos corresponde a uno que aporta las mejores soluciones a los problemas de multioptimización dentro de la migración $(20)^{23}$. Existe también otro enfoque ortogonal que se encarga de resolver los siguientes problemas:

- Los algoritmos de agrupación existentes pueden dividir el sistema heredado en clústeres diferentes.

- La precisión de la partición del cluster debe mejorarse aún más ${ }^{24}$.

Otro aporte de los autores, es la identificación de un catálogo de refactorizaciones arquitectónicas para el diseño de aplicaciones en la nube. Este catálogo de refactorización fue compilado a partir de la revisión de patrones de nubes, así como experiencias de proyectos. El conocimiento de la nube y las plantillas de apoyo se han validado a través de la investigación y la implementación en cooperación con los profesionales en el tema ${ }^{25}$. Uno de los problemas existentes es el consumo de energía, el cual es un punto notable de complicación en el trabajo computacional. Par ello se propone una solución para diagnosticar sobrecarga de host y eliminarla a fin de mejorar el rendimiento de la migración de las máquinas virtuales ${ }^{26}$.

Asimismo, se ha encontrado otro aporte de reducción de tiempo de pre-copia de la migración a la nube. El cual propone un mecanismo de predicción de memoria, el cual puede elegir cargas para migrar en la fase de pre-copia. La propuesta es capaz de decidir el mejor momento para optimizar la migración de memoria y aumentar la velocidad de migración ${ }^{27}$. Entre otros aportes, se tiene un enfoque basado en la Red de Petri, el cual evalúa la actividad y exactitud de los sistemas. Los resultados de la simulación sugieren que la migración de tareas basada en CCPN (Red de Petri) puede combinar la minimización del consumo de energía con un buen rendimiento general ${ }^{28}$.

\footnotetext{
${ }^{23}$ Cfr. ABDELZAHIR A., DAYANG N. A. JAWAWI, IMRAN GHANI, ABUBAKAR ELSAFI (2015)

24 Cfr. Zhao Junfeng, Zhou Jiantao, Yang Hongji, Liu Guoping (2015)

25 Cfr. Olaf Zimmermann (2017)

${ }^{26}$ Cfr. Arjmandi Mona, Balouchzahi Nik, Raahemifar K., Fathy M., Akbari Ahmad (2016)

${ }^{27}$ Cfr. Tin-Yu Wu, Nadra Guizani, Jhih-Siang Huang (2017)

${ }^{28}$ Cfr. Lianzhang Zhu, Shouchao Tan, Weishan Zhang, Yong Wang, and Xiwei Xu (2016)
} 


\section{Tópico 6: Métodos para la migración a la nube}

Entre los métodos existentes, los autores mencionan un método de migraciones paralelas para disminuir el tiempo requerido para la migración de las máquinas virtuales, lo que reducirá el tiempo de estas, las cuales se dan en cadena ${ }^{29}$. Tocando el tema de métodos para optimizar el tiempo, se encuentra el aporte que propone un método para identificar los ciclos de carga de trabajo en una máquina virtual, con la necesidad de optimización de recursos computacionales para realizar las cargas ${ }^{30}$. Otro aporte para el tema de las cargas de trabajo, trata de proponer técnicas distribuidas de resolución de problemas para la gestión de cargas en centros de datos soportados por la migración en vivo de máquinas virtuales y una nueva heurística de equilibrio de carga que migra las máquinas virtuales que causan el mayor desequilibrio ${ }^{31}$.

Otro problema que encontramos es cuando el usuario al usar el centro de datos de manera extrema da lugar a una sobrecarga y falta de eficiencia energética en los centros de datos. En este trabajo los nodos de computación en la nube del Datacenter se reducen por el método de migración. El uso inactivo de la máquina virtual consume electricidad extrema y emite dióxido de carbono que da lugar a un ambiente dañino. Para superar la sobrecarga del host se propone el método Inter-Migración que cambia los nodos activos y los que duermen en los centros de datos ${ }^{32}$.

Uno de los otros aportes propone un ataque DDOS, que puede congestionar la red de comunicación interna de los servidores en cluster y consumir sus recursos. Evaluando posibles amenazas ocultas detrás de la máquina virtual normal. Migrando y analizando las fallas de los sistemas de detección de intrusos existentes / sistema de prevención para la defensa del ataque $\mathrm{CDF}^{33}$. A su vez, proponen otro método para minimizar el retraso de los servicios, el método contiene un enfoque dual en computación y comunicación de los elementos ${ }^{34}$. Por otro lado, otro aporte es la clasificación de las estrategias de migración, luego se revisan y comparan las investigaciones relacionadas con cada estrategia de migración. Además, se encuestan las herramientas de desarrollo

\footnotetext{
${ }^{29}$ Cfr. Kim Changhyeon, Jeon Changho, Lee Wonjoo, Yang Sungil (2015)

${ }^{30}$ Cfr. A. Baruchi, E. T. Midorikawa and L. M. Sato (2015)

${ }^{31}$ Cfr. J. Octavio Gutierrez-Garcia and Adrian Ramirez-Nafarrate (2015)

32 Cfr. TAMILVIZHI.T, PARVATHA VARTHINI.B. (2017)

33 Cfr. Yichuan Wang, Jianfeng Ma, Di Lu, Xiang Lu, Liumei Zhang (2014)

${ }^{34}$ Cfr. Tiago Gama Rodrigues, Katsuya Suto, Hiroki Nishiyama and Nei Kato (2016)
} 
relacionadas. Sobre la base de los logros existentes en la investigación y el estado de la aplicación, se identifican algunos trabajos futuros que incluyen metodología holística, rediseño y adaptación a la aplicación para migración especial, refactorización de la arquitectura, entorno de desarrollo integrado, etc. ${ }^{35}$.

\section{Estudios}

\section{BGM-BLA: A NEW ALGORITHM FOR DYNAMIC MIGRATION OF VIRTUAL MACHINES IN CLOUD COMPUTING}

\section{Motivación}

La motivación de Tao et al. Se basa en las soluciones anteriores de algoritmos de asignación de máquinas virtuales a nodos físicos que carecen de evaluación del factor del consumo de energía. Ellos esperan proponer el primer algoritmo de asignación de máquinas virtuales a nodos físicos que tome en consideración tanto la energía que representa a la cantidad consumida por el nodo físico como a la energía del poder computacional del nodo físico.

\section{Aporte}

Los autores proponen BGM-BLA (Binary Graph Matching-based Bucket-code Learning Algorithm), con la intención de resolver los problemas de migración divididos en 2 partes: Formar grupos de VM's y determinar la mejor manera para ubicar los grupos en nodos físicos. El algoritmo planteado tiene los siguientes 4 pasos principales:

- Generating Bucket Codes

- Consiste en asignar valores a las máquinas virtuales para de esa manera agruparlos en grupos VM's.

- Evaluating the three objectives of every bucket code by the KM algorithm

- El algoritmo Kuhn-Munkres (KM) permite ubicar dichos grupos de VM's en nodos físicos.

- Learning and Mutation

\footnotetext{
${ }^{35}$ Cfr. A. Baruchi, E. T. Midorikawa and L. M. Sato (2015)
} 
- La primera parte consiste en agrupar los códigos con la primera parte similar, evaluando de esta manera los códigos se puede aprender cual se asocia con cual. La segunda parte consiste en modificar la segunda parte de los códigos a un valor apropiado.

- Outputting the pareto set of solutions

- Se procede a modificar el diagrama de pareto correspondiente

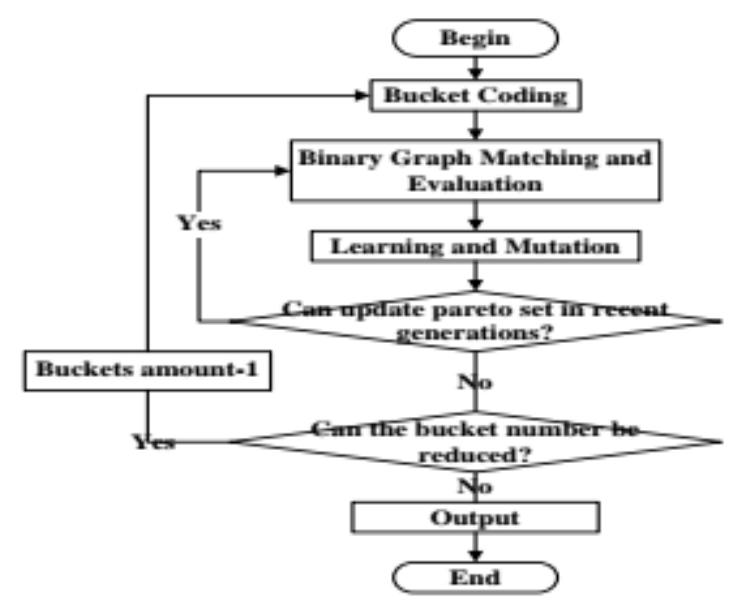

Ilustración 4: Flujo de BGM - BLA

\section{Validación}

Para la validación se realizó una comparación entre el algoritmo BGM-BLA con otros algoritmos como NSGAII, BGM-CA, y se comparó sus soluciones encontradas, lo que demostró que los algoritmos BGM-BLA incrementa el tamaño de los errores, lo que lo hace mejor que los otros 2 algoritmos mencionados anteriormente.

\section{AUTOMATION AND ORCHESTRATION FRAMEWORK FOR LARGE-SCALE ENTERPRISE CLOUD MIGRATION}

\section{Motivación}

La motivación de Hwang et al. Se basa en soluciones que los frameworks de migración existentes se centran en migración de aplicaciones legacy hacia el ambiente de Software as a Service (SaaS). Además, los servidores on-premises corren en diferentes plataformas, 
diferente hardware físico y varios hipervisores que involucran diferentes formatos de imágenes.

\section{Aporte}

Los autores proponen Cloud Migration Orchestrator (CMO), el cual es un framework basado en principios de Business Process Management (BPM), cuyo objetivo es automatizar y orquestar las actividades de migración, entre las que se incluyen descubrimiento, análisis, configuración de la pre-migración, ejecución de la migración, configuración de la post-migración y aseguramiento de la calidad.

Los beneficios de usar BPM son el monitoreo, compartimiento del contexto, y orquestación completa del proceso; esto permite la variabilidad de distintos escenarios de migración que puede soportar CMO.

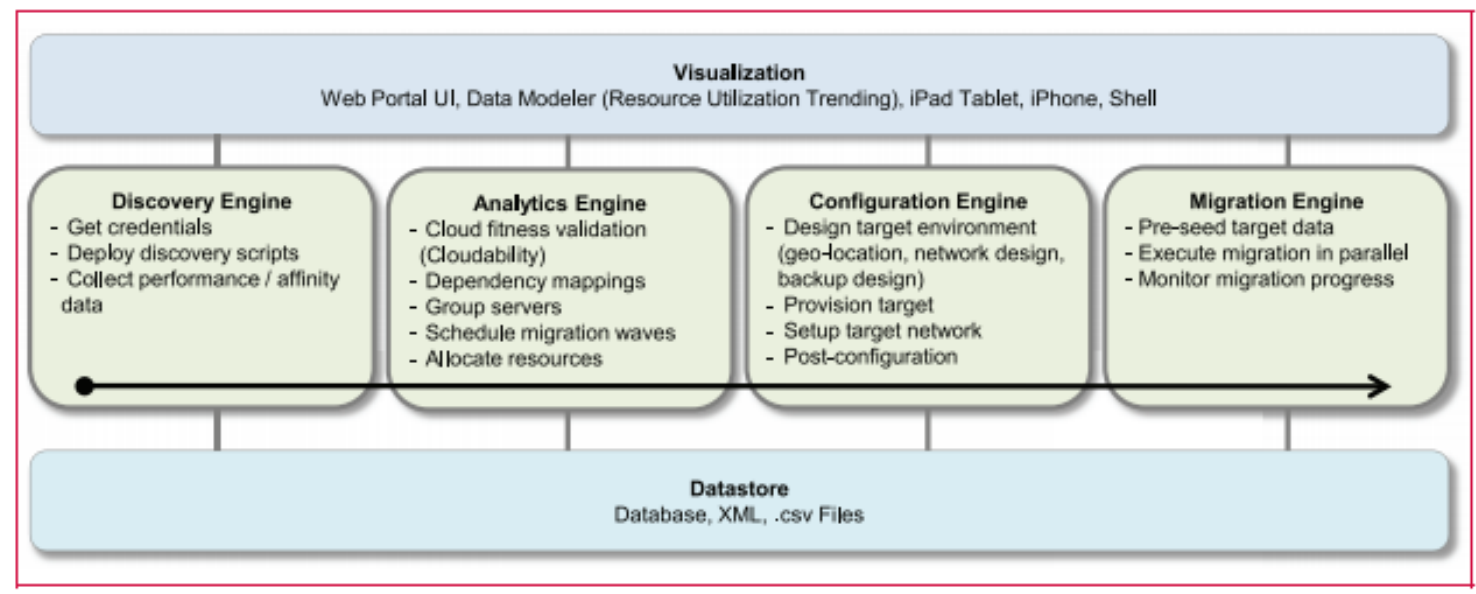

Ilustración 5: CMO Framework

\section{Validación}

El framework propuesto, Cloud Migration Orchestrator (CMO), se puso a prueba en servidores virtuales de alta demanda, primero se probó con 25 servidores con datos ingresados, y otros 25 sin datos; luego de haber realizado los pasos solicitados por el framework, se obtiene el siguiente resultado: 


\begin{tabular}{|c|c|c|c|c|c|}
\hline No. VMs & Total size (GB) & Transfer size (GB) & Average time & $\begin{array}{l}\text { Minimum } \\
\text { time }\end{array}$ & $\begin{array}{l}\text { Maximum } \\
\text { time }\end{array}$ \\
\hline $\begin{array}{l}25 \text { VMs ( } 10 \text { Linux and } 15 \\
\text { Windows, no data filled) }\end{array}$ & 750 & 171.75 & $3: 07: 00$ & $2: 43: 34$ & $3: 27: 33$ \\
\hline $\begin{array}{l}25 \text { VMs ( } 10 \text { Linux and } 15 \\
\text { Windows, same data filled) }\end{array}$ & 750 & 271.3 & $4: 10: 25$ & $3: 47: 32$ & $4: 54: 45$ \\
\hline $1 \mathrm{VM}$ (data filled) & 200 & 168.54 & $3: 01: 22$ & $2: 42: 47$ & $4: 12: 33$ \\
\hline
\end{tabular}

Ilustración 6: Comparación de tiempo de transferencia de datos

Como conclusión, se demostró que CMO provee una instalación automatizada en la cual no es necesaria la intervención humana.

\section{SUPPORT VECTOR MACHINE APPROACH FOR VIRTUAL MACHINE MIGRATION IN CLOUD DATA CENTER}

\section{Motivación}

En el artículo en mención consideran en estabilizar el uso promedio de las máquinas virtuales y máquinas físicas en el centro de datos en la nube. Los recursos que se solicitan y algunos servicios específicos se van actualizando, lo que causa que las máquinas virtuales agoten los recursos de las máquinas físicas en donde se encuentran montadas.

\section{Aporte}

Desde la línea 2 hasta la línea 6, todas las máquinas virtuales de tipo A se colocan en las máquinas físicas. El mismo procedimiento anterior, el tipo B de las máquinas virtuales se asignan a las máquinas físicas en la línea 7 a la línea 11. De la línea 12 a la línea 15, asignamos las máquinas virtuales de tipo $\mathrm{D}$ a las físicas, que se asignaron a máquinas virtuales de tipo A en la línea 2 a la línea 6, hasta que no haya más recurso. 
Para máquinas virtuales de tipo D, de la línea 16 a la línea 18, las máquinas virtuales de tipo $\mathrm{C}$ son asignados a las físicas, que las virtuales de tipo B colocadas anteriormente. Si se utilizan maquinas física para máquinas virtuales de tipo $\mathrm{C}$, las virtuales de tipo $\mathrm{C}$ se asignan a otras físicas en las líneas 19 a 24, hasta que todas las máquinas virtuales de tipo C se hayan asignado. De la línea 25 a la línea 29, si las virtuales de tipo D aún no se asignan, asignamos la que se mantuvo de tipo D virtual a física. Además, las máquinas virtuales no asignadas de tipo D se colocan a otras físicas en la línea 30 a la línea 33. Y de este modo se asegura que todas las máquinas virtuales sean asignadas.

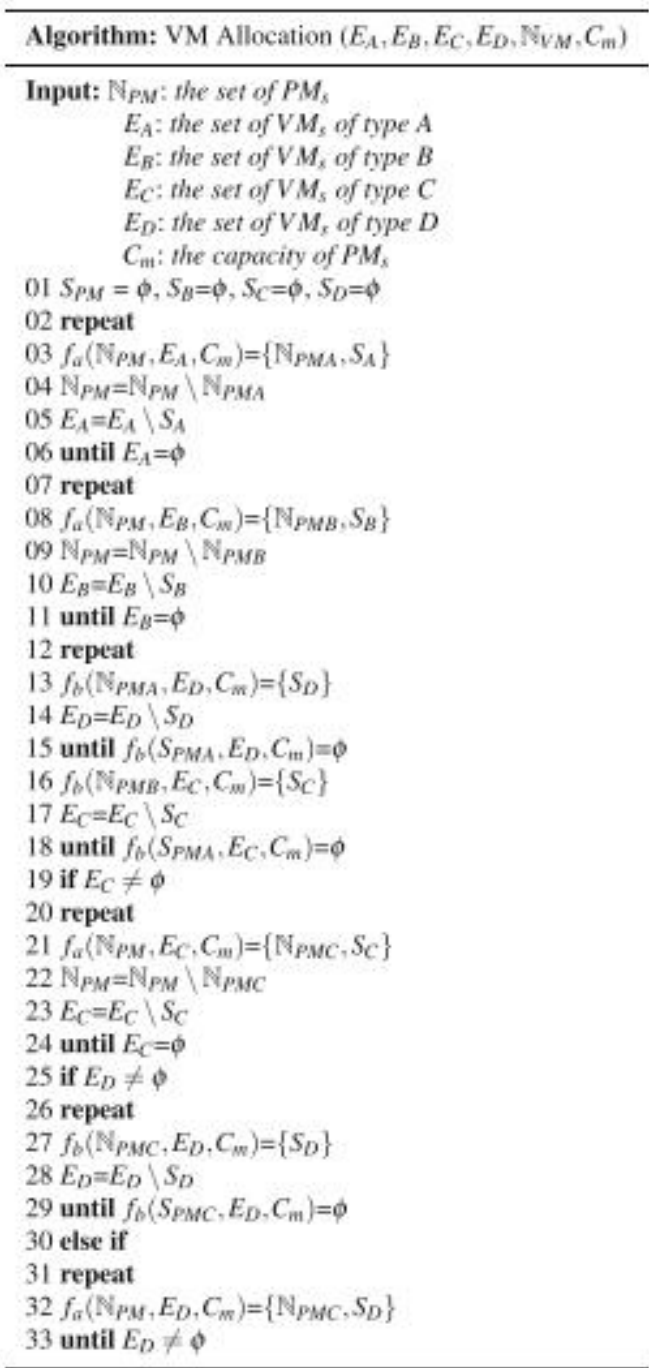

Ilustración 7: Algoritmo de locación de las maquinas virtuales 


\section{Validación}

En el algoritmo propuesto de asignación de máquinas virtuales, asignamos las virtuales de acuerdo a sus tipos, que se juzgan con el requisito máximo durante el tiempo de entrenamiento.

En primer lugar, asignan respectivamente las virtuales de tipo A y las virtuales de tipo B a las Maquina Física 1, Maquina Física 2, Máquina Física 3, Máquina Física 4 y Máquina Física 5. Luego distribuimos las virtuales de tipo D a la física 1 y física 2 . En esta fase, asignamos las virtuales de tipo $\mathrm{C}$ a las virtuales 3 y 4 . Por último, distribuimos las máquinas virtuales restantes (VM10 en este caso) para la máquina física 5, que tiene recursos suficientes para colocar la VM10. La asignación de máquinas virtuales se basa en el requisito máximo durante el tiempo de entrenamiento.

\section{COST-MINIMIZING DYNAMIC MIGRATION OF CONTENT DISTRIBUTION SERVICES INTO HYBRID CLOUDS}

\section{Motivación}

Utilizar la nube privada existente del proveedor de aplicaciones, para atender solicitudes volátiles con tiempo de respuesta del servicio. Lo cual garantizará en todo momento, el tiempo que incurrirá en el coste operativo mínimo.

\section{Aporte}

Diseñar un algoritmo de distribución conjunta de contenido y carga que minimice el costo operacional general a lo largo del tiempo. El cual estará sujeto a restricciones de tiempo de respuesta del servicio. El diseño propuesto está basado en la teoría de optimización de Lyapunov, donde la minimización de costos y la garantía de tiempo de respuesta se logran simultáneamente mediante una programación eficiente de migración de contenido y despacho de solicitud entre el centro de datos. 
La optimización de Lyapunov proporciona un marco para diseñar algoritmos con un rendimiento arbitrariamente cercano al rendimiento óptimo a largo plazo del sistema, sin necesidad de ninguna información futura. Ha sido ampliamente utilizado en enrutamiento y asignación de canales en redes inalámbricas, y sólo recientemente se ha introducido para abordar los problemas de asignación de recursos en algunos otros tipos de redes. Se está adaptando las técnicas de optimización de Lyapunov en la configuración de una nube híbrida, para resolver de forma dinámica y conjunta los problemas óptimos de replicación de contenido y distribución de carga.

\section{Validación}

El proveedor de aplicaciones despliega uno o varios servidores web que proporcionan servicio de portal de la aplicación de distribución de contenido, de forma centralizada o distribuida. El portal agrega las peticiones de los usuarios y envía la información de la solicitud recopilada a un centro de control, que ejecuta nuestro algoritmo periódicamente.

\section{CLOUDGENIUS: A HYBRID DECISION SUPPORT METHOD FOR AUTOMATING THE MIGRATION OF WEB APPLICATION CLUSTERS TO PUBLIC CLOUDS}

\section{Motivación}

La motivación de Menzel et al. Consiste en que las aplicaciones web tradicionales requieren que se les provea hardware para los picos de carga. Sin embargo, cuando la carga no es tan alta, resulta muy caro el mantener ese hardware que está siendo poco usado.

\section{Aporte}


Se propone una arquitectura de servidores en cluster a una infraestructura cloud, la cual es llamada CloudGenius. La migración de aplicaciones debe contemplar muchas repeticiones y reconsideraciones.

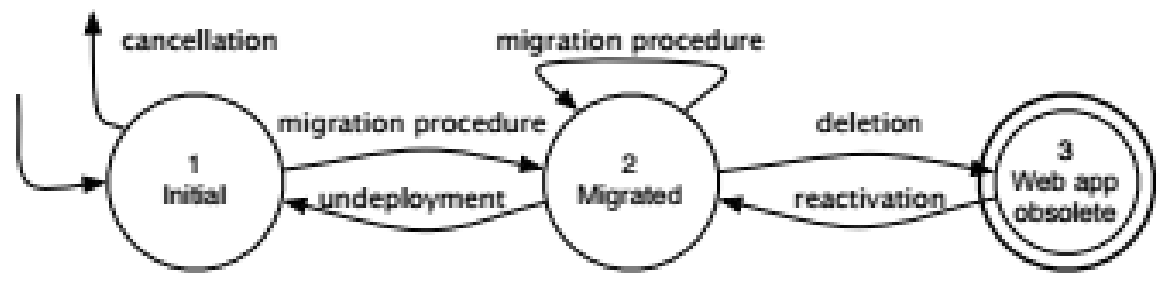

Ilustración 8: Estados en migración a la nube evolutiva

Cluster de aplicaciones web comprenden el balanceador de cargas, servidor de base de datos y componentes web interconectados; el modelo de CloudGenius consiste en lo siguiente:

\begin{tabular}{|l|l|}
\hline Parameter & Description \\
\hline$\tilde{F}$ & cluster to migrate consisting of components $C$ \\
$C=\left\{c_{1}, \ldots, c_{l}\right\}$ & set of $l$ software components \\
$A=\left\{a_{1}, \ldots, a_{m}\right\}$ & set of $m$ cloud VM images \\
$S=\left\{s_{1}, \ldots, s_{n}\right\}$ & set of $n$ cloud infrastructure services \\
$P=\left\{p_{1}, \ldots, p_{o}\right\}$ & set of $o$ cloud providers \\
$D=\left\{d_{1}, \ldots, d_{q}\right\}$ & set of $q$ image-service compatibilities $\left(a_{i}, s_{j}\right)$ \\
$\left.E=\left\{\left(a_{i}, a_{j}\right) \mid a_{i}, a_{j} \in A\right)\right\}$ & set of inter-image compatibilities \\
$\left.F=\left\{\left(s_{i}, s_{j}\right) \mid s_{i}, s_{j} \in S\right)\right\}$ & set of inter-service compatibilities \\
$I=\left\{\left(c_{i}, c_{j}\right) \mid c_{i}, c_{j} \in C\right\}$ & set of relations between components \\
$R_{A}=\left\{r_{A, 1}, \ldots, r_{A, r_{A}}\right\}$ & set of $r_{A}$ cloud image requirements \\
$R_{S}=\left\{r_{S, 1}, \ldots, r_{S, r_{S}}\right\}$ & set of $r_{S}$ cloud service requirements \\
$R_{c_{h}, X},\left\{r_{X, 1}, \ldots, r_{X, r_{X}}\right\}$ & set of $r_{X}$ combination requirements for $c_{h}$ \\
$\tau_{h}$ & cloud image $a_{i}$, service $s_{j}$, provider $p_{k}$, or combination $x_{l}$ as $\tau_{h} \in A \cup S \cup P \cup X$ \\
$\hat{A}_{\tau_{h}}=\left\{\alpha_{\tau_{h}, 1}, \ldots, \alpha_{\tau_{h}, t}\right\}$ & set of $t$ numerical attributes of $a_{i}, s_{j}, p_{k}, x_{l}$ \\
$\hat{B}_{\tau_{h}}=\left\{\beta_{\tau_{h}, 1}, \ldots, \beta_{\tau_{h}, u}\right\}$ & set of $u$ non-numerical attributes of $a_{i}, s_{j}, p_{k}, x_{l}$ \\
$\chi(\alpha)$ & value of numerical attribute $\alpha$ in CloudGenius database \\
$\chi(\beta)$ & value of non-numerical attribute $\beta$ in CloudGenius database \\
$v_{\tau_{h}}$ & value of h-th $\tau$ calculated with $\left(M C^{2}\right)^{2}$ \\
$X_{c_{h}}=\left\{x_{l}=\left(a_{i}, s_{j}\right) \mid a_{i} \in A, s_{j} \in S\right\}$ & set of cloud image and service combinations for $c_{h}$ \\
$N_{o} u t=\left\{n_{o u t},\left(c_{i}, c_{j}\right) \mid c_{i}, c_{j} \in C\right\}$ & set of outgoing traffic between components (in byte) \\
$\left.N_{i} n=\left\{n_{i n,\left(c_{i}, c_{j}\right)} \mid c_{i}, c_{j} \in C\right)\right\}$ & set of incoming traffic between components (in byte) \\
\hline
\end{tabular}

Ilustración 9: Modelo formal de CloudGenius

\section{Validación}

Se utilizó CloudGenius para migrar una aplicación web a cloud, para lo cual se tomó en cuenta a los componentes relacionados a la aplicación, dicha aplicación consistía de los 
siguientes componentes: 2 servidores de aplicación Tomcat, un servidor de base de datos MySQL, y la propia aplicación. Los resultados obtenidos fueron los siguientes:

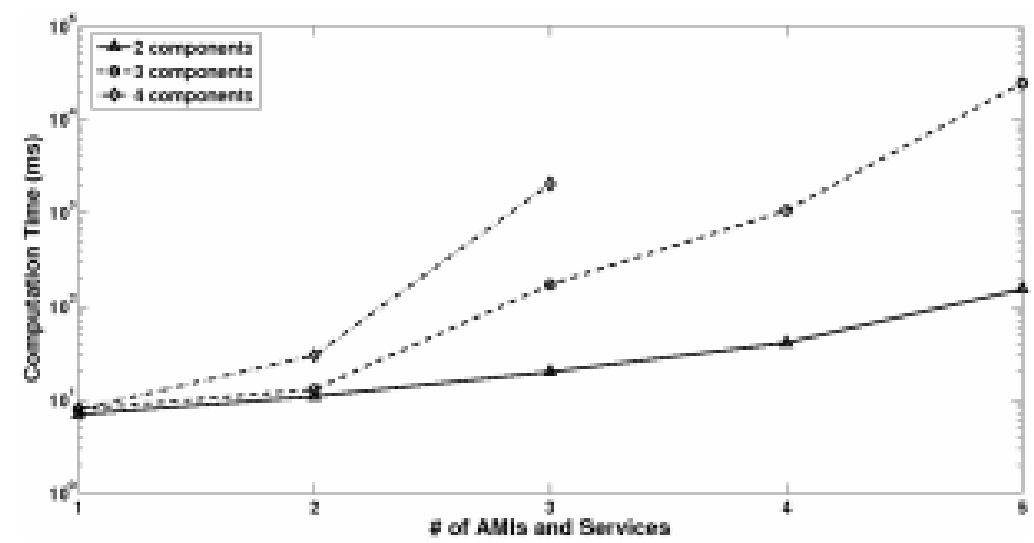

Ilustración 10: Complejidad de tiempo en computación paralela

Se demostró que la aplicación funcionó correctamente en cloud junto a sus componentes.

\section{CORRELATION BASED VIRTUAL MACHINE MIGRATION IN DYNAMIC CLOUD ENVIROMENTS}

\section{Motivación}

La virtualización del hardware permite a los proveedores de la nube proveer una variedad de máquinas virtuales que operan de manera similar a los dispositivos de computación real con un sistema operativo y otro software. Estas máquinas virtuales proporcionan flexibilidad al permitir diferentes entornos de computación en la misma máquina host subyacente y también aumentar la eficiencia (por ejemplo, reducir el costo) compartiendo los recursos del sistema subyacente.

\section{Aporte}


Se propone una estrategia integrada de migración de máquinas virtuales. En primer lugar, se establece un mecanismo de migración suave basado en acuerdos de nivel de servicio para reducir significativamente el número de migraciones de máquinas virtuales. A continuación, se desarrolla dos algoritmos para resolver los problemas de las máquinas y selección de servidores, en los que se utiliza la correlación entre las máquinas virtuales y los servidores para identificar las máquinas virtuales adecuadas a migrar y los servidores de destino para ellas.

- Se utiliza las siguientes métricas para evaluar el rendimiento de nuestros algoritmos propuestos.

- El número de migraciones de máquinas virtuales: es el número total de migraciones en todos los servidores. Esta métrica sirve como una indicación de la frecuencia de migración.

- Consumo de energía: Esta métrica se usa para medir la energía total consumida por los servidores a medida que aumenta el número de VMs.

- El número promedio de migraciones de VM que se producen por intervalo de tiempo: Esta métrica se utiliza para evaluar el número promedio de migraciones de VM que se deben realizar en cada intervalo de tiempo. 


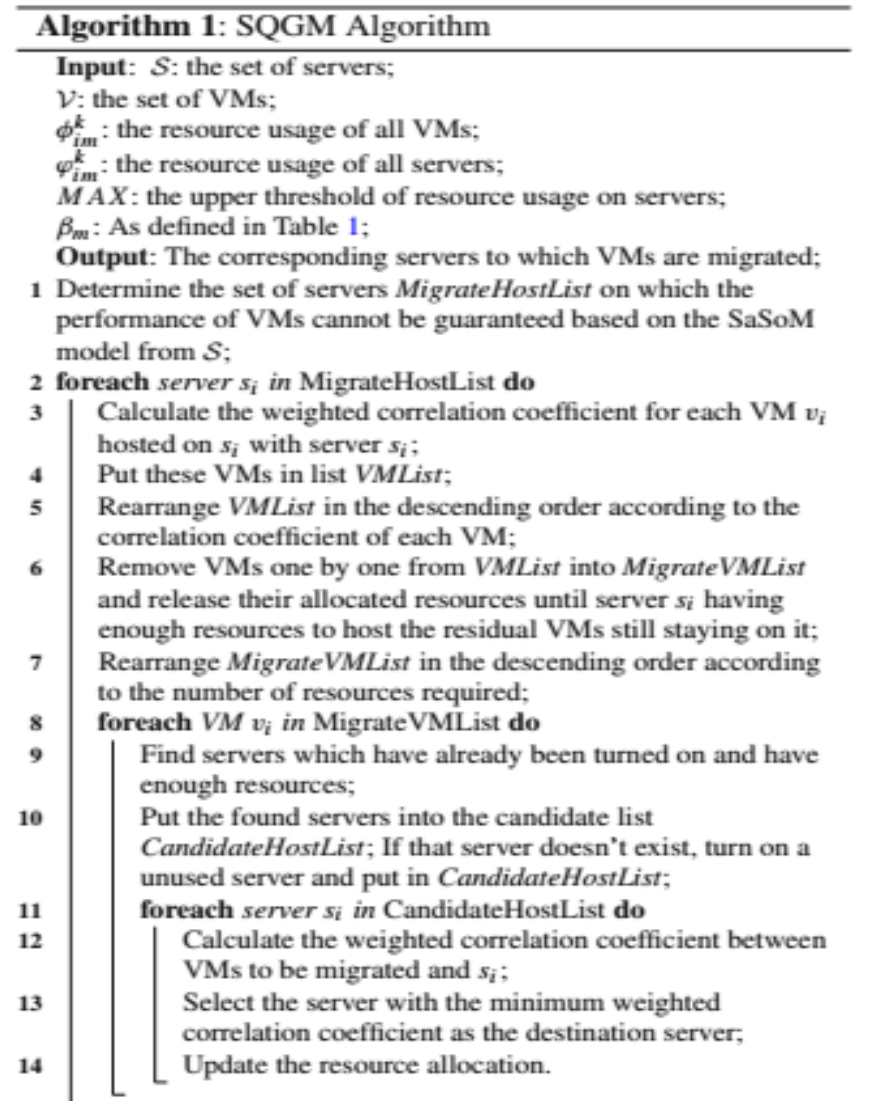

Ilustración 11: Algoritmo SQGM 


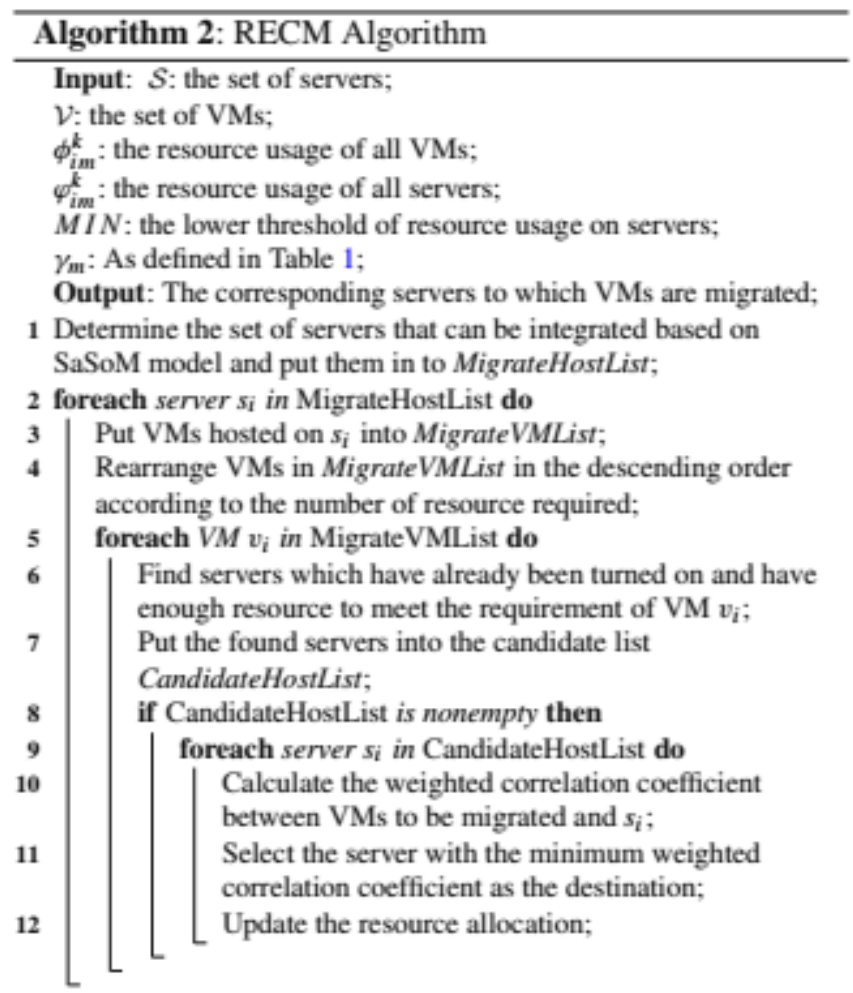

Ilustración 12: Algoritmo RECM

\section{Validación}

Se ha realizado experimentos de simulación exhaustivos para evaluar el rendimiento de los algoritmos propuestos. Los resultados experimentales muestran que nuestros algoritmos de migración VM propuestos superan a otras migraciones tradicionales de máquinas virtuales para reducir el costo de migración de estas y mejorar la utilización de servidores físicos en data center. 


\section{CAPITULO 5: DESARROLLO DEL MODELO DE MIGRACIÓN A LA NUBE DE LOS SERVIDORES DE UN DATA CENTER}

El siguiente capítulo muestra el detalle del trabajo realizado con la finalidad de plantear el Modelo de Migración a la nube de los servidores de un data center. 


\section{Referencias}

\section{Fuentes Primarias}

En la literatura, como primera fuente tenemos el marco de trabajo de Bazi Hamid, el cual presenta un framework de migración a la nube. Extrae conceptos de investigaciones anteriores y los clasifica en categorías. Al realizar su estudio en 657 artículos, los conceptos obtenidos son clasificados en categorías y subcategorías relacionadas. Con ello su modelo se divide en 7 fases (categorías) y 15 subcategorías. Se propone un modelo de madurez para mejorar el proceso migratorio. Ya que al utilizar un marco fiable para la migración aseguran a los administradores la mitigación de los riesgos en la tecnología de cloud computing así como integridad junto con su simplicidad. ${ }^{36}$

Como segunda fuente analizada, tenemos el trabajo de Gholami Mahdi, quien propone como objetivo del proyecto una revisión detallada de las migraciones a cloud existentes, desde la perspectiva como modelo de proceso. Para ello utilizan un marco de evaluación de los enfoques existentes para destacar las características más importantes y resaltantes. Como resultado se tiene un listado de actividades, recomendaciones, buenas prácticas, técnicas y de preocupaciones comunes en el proceso de la migración a la nube. ${ }^{37}$

Como tercera fuente, el trabajo de Jung-Feng Zhao propone un estudio. Que se basa en analizar los logros de la investigación y el estado de la aplicación, dividir los métodos de migración existentes en tres estrategias según los modelos de servicio en la nube integralmente. Diferentes procesos deben ser considerados para diferentes estrategias de migración, y diferentes tareas son involucradas en consecuencia. Estas estrategias que nos brindan son consideradas en las recomendaciones/buenas prácticas que nos deja este artículo. $^{38}$

\footnotetext{
${ }^{36}$ Bazi Hamid

${ }^{37}$ Gholami Mahdi

${ }^{38}$ Jung-Feng Zhao
} 


\section{Fuentes Secundarias}

Para migrar a la nube es necesario tener en cuenta diferentes factores, este segmento del presente documento presenta algunas recomendaciones y buenas prácticas para la migración a la nube, según algunos proveedores.

\section{Según IBM}

Antes de migrar a la nube se deben evaluar diferentes factores que son claves en el transcurso del proyecto. En primer lugar, es importante señalar que no todo tiene o puede estar en la nube, por tal motivo se tiene que identificar qué cosas se deben migrar y cuáles no, evaluando las ventajas y desventajas de los mismos.

Para ello, IBM recomienda que para migrar se debe seguir la siguiente guía:

- Para una cloud pública, las cargas de trabajo de infraestructura suelen ser las más apropiadas, entre estas se puede mencionar:

- Conferencias web, audio y video.

- Servicio de HelpDesk.

- Infraestructura para entrenamiento y demostración.

- Almacenamiento.

- Servidores.

- Para una cloud privada, las bases de datos y aplicaciones de trabajo suelen ser las más importantes, entre estas se puede mencionar:

- Data mining.

- DataWareHouse y DataMart.

- Bases de datos transaccionales.

- Aplicaciones de la industria.

- Aplicaciones ERP.

Además, los siguientes no deberían incluirse en cloud:

- Datos confidenciales

- Software de terceros todavía no virtualizado 
- Procesos y transacciones complejas

- Regulación sensible

Las barreras de salida también deben ser consideradas, debido a que, aunque las barreras de entradas al modelo cloud pública son bajas, las barreras de salida podrían no serlo. Por tal motivo se deben evaluar diferentes proveedores en busca de uno que ofrezca alta portabilidad.

Algunas organizaciones o países presentan temas regulatorios y normativos que no les permiten migrar ciertos datos o aplicaciones a la nube, estos casos deben ser mapeados antes de la migración

Evaluar a qué tipo de cloud se desea migrar es necesario, ya sea público o privado. Las ventajas de ambos modelos de cloud, es principalmente que el público es flexible y económico; mientras el privado permite mayor control, seguridad y privacidad de información crítica.

Las siguientes preguntas ayudan a definir un plan de migración:

- ¿Cuál es la mejor infraestructura para cada carga de trabajo?

- ¿Existen cargas de trabajo que deberían quedar al margen de la migración a la nube?

- ¿Cómo cuantificamos las ventajas de la migración a la nube?

- ¿Cómo priorizamos la migración de cargas de trabajo?

- ¿Cómo realizamos la migración sin interrumpir la actividad empresarial corriente?

- ¿Existe una metodología lógica para desentrañar estas cuestiones?

Y se debe adicionar las siguientes preguntas cuando se trata específicamente de migración de aplicaciones:

- ¿En qué ambiente operan (Windows, Linux, etc.)? ¿Qué requisitos exigen?

- ¿Qué protocolos de comunicación usan las aplicaciones? ¿Qué tipo de arquitectura tienen (Cliente - Servidor, P2P)? ¿Están basadas en la web?

- ¿Qué aspectos normativos hay que evaluar en caso que existan? 
- ¿Cuáles son los requisitos de seguridad?

- ¿Es necesario crear una nueva arquitectura de aplicación?

Los datos y sistemas necesitan una preparación previa a la migración y esto ha de contemplarse en el plan de migración. Este paso se estructura en torno a tres momentos:

- Establecimiento de los requisitos de calidad: aplicables a cada elemento, sus atributos e interrelaciones dentro del sistema fuente. Implica la definición de las reglas de calidad y requiere de la recogida de incidencias para su monitorización y evaluación.

- Perfilado: en base a los formatos existentes y a los modelos de estructura que imperan en destino, se debe preparar los datos de origen para asegurar que, no sólo su calidad es suficiente, sino que están preparados para el cambio. Hay que determinar también el modo de proceder con los datos que no cumplan con estos requisitos.

- Limpieza: puede llevarse a cabo en una o dos etapas, pero siempre se ha de sujetar a verificación, para comprobar que los resultados obtenidos son los esperados.

\section{Según Hewlett Packard}

Las empresas se enfrentan a una amplia diversidad de opciones y decisiones para los modelos de entrega en la nube. Existen varias alternativas de nube: pública, privada e híbrida. Cada una tiene sus méritos, por lo que es importante encontrar la estrategia óptima que proporcione el resultado correcto para la empresa y las eficacias operativas de TI adecuadas. He aquí algunas de las preguntas esenciales para que las TI empresariales exploren:

- ¿Cuál es la mejor infraestructura para cada carga de trabajo?

- ¿Existen cargas de trabajo que deberían quedar al margen de la migración a la nube?

- ¿Cómo cuantificamos las ventajas de la migración a la nube?

- ¿Cómo priorizamos la migración de cargas de trabajo?

- ¿Cómo realizamos la migración sin interrumpir la actividad empresarial corriente?

Hewlett Packard plantea una metodología que se divide 5 fases; Descubrimiento, Idoneidad, Asignación, Migración y Capacitación, los cuales proporcionan análisis detallados de cargas de trabajo de servidor existentes y decisiones relativas a la mezcla correcta de servicios de nube para cualquier empresa. 


\section{Descubrimiento}

La primera fase es la de Descubrimiento, que busca identificar las cargas de trabajo y los servidores en los que se ejecutan (físicos y virtuales), los dispositivos de almacenamiento y bases de datos utilizados, los patrones de consumo de recursos, las redes y componentes, las relaciones y las dependencias entre todas estas entidades.

\section{Idoneidad}

Debido a que no todo tiene que ir a la nube, se debe realizar una evaluación de las cargas de trabajo de las aplicaciones para establecer qué cargas de trabajo deberían moverse a la nube y cuáles no, para ello se debe tomar en cuenta qué beneficio se obtiene al pasarlo a la nube, si es posible realizar la migración o no.

Esto permite determinar uno de los cuatro resultados posibles para cada carga de trabajo de la aplicación:

- Migrar la carga de trabajo a la nube no genera beneficios.

- No es posible migrar la carga de trabajo a la nube por alguna de las siguientes razones: económicos, técnicos o funcionalidad.

- No es prioritario mejorar la carga de trabajo a la nube.

- La carga de trabajo es candidata para la migración.

El resultado final de esta fase es una lista de cargas de trabajo calificadas, capaces de ejecutarse en nubes de destino y listas para una migración física.

\section{Asignación}

En este punto se deben realizar nuevas revisiones de la lista de cargas de trabajo candidatas para seleccionar el mejor modelo de entrega de la migración, teniendo en cuenta que las ventajas de cada modelo explicadas anteriormente.

\section{Migración}

Consiste en mover las cargas de trabajo desde el punto de origen hasta el punto de destino. Esta actividad debe realizarse de a pocos, una a una. En muchas situaciones se deben 
extraer desde el mismo entorno de producción, lo que altera el funcionamiento normal la organización.

\section{Capacitación}

Validar las conexiones, los niveles de servicio y las consideraciones de rendimiento de aplicaciones recién migradas. La fase de capacitación responde a preguntas sobre si todas las cargas de trabajo de destino se migraron con éxito y si se alcanzó el valor esperado.

\section{Según Red Hat}

Consultoría Red Hat ha desarrollado un marco de prestación de soluciones que permite migrar a la nube en 4 fases:

\section{Descubrimiento}

Consiste en una reunión exploratoria para definir el alcance del problema, el enfoque de la solución y las recomendaciones de los próximos pasos, esto ayuda a:

- Alinear las necesidades tecnológicas predominantes, experiencias similares y resultados esperados.

- Detallar el dominio de general del problema, incluido el estado actual, el estado objetivo, y las oportunidades para llevar a cabo proyectos.

\section{Diseño}

Se realiza un taller interactivo y colaborativo con el personal clave para desarrollar un plan integrado, para la migración. Los componentes de este taller se centran en los siguientes elementos:

- Análisis del estado actual de la arquitectura y de las prácticas y de las prácticas organizativas para la migración

- Asignación de estrategia entre el personal, procesos y tecnología para definir un enfoque sólido y hacer evolucionar el enfoque, la implementación y la gestión de servicios de infraestructura.

- Definición de la arquitectura a alto nivel para hacer frente a los requisitos de entorno objetivo y la cartera de carga de trabajo. 


\section{Implementación}

A partir del trabajo realizado en el taller de diseño, se realizan proyectos flexibles para:

- Construir y probar cada infraestructura de migración a la nube adaptable.

- Preparar las cargas de trabajo por grupos de atributos significativos, definir y probar los procesos de automatización de migración adaptados

- Migrar mediante la programación y la migración interactiva de cargas de trabajo para llevar las aplicaciones de usuario final a su nueva infraestructura de forma eficiente, según lo demanden las necesidades empresariales.

- Gestionar la implementación de un catálogo de autoservicios y el aprovisionamiento automático para abordar fácilmente las operaciones en curso

\section{Aplicación}

Se debe seguir contribuyendo al éxito durante y después de la implementación, con las siguientes actividades:

- Tutorizar sus operaciones, equipos de gobernabilidad y desarrollo para adoptar enfoques colaborativos.

- Capacitar y certificar sus equipos en tecnologías actuales y emergentes.

\section{Según Amazon Web Services}

Para Amazon, la planificación temprana, la comunicación y la aceptación son esenciales. Entender el tiempo, costo, disponibilidad, etc. es clave y será diferente para cada organización. Al definir el modelo de migración, las organizaciones deben tener una estrategia clara, mapear un cronograma de proyecto realista, limitar el número de variables y dependencias para la transición de aplicaciones locales a la nube. A lo largo del proyecto, generar impulso con las principales reuniones periódicas y presentación de informes para examinar el progreso y el proyecto para mantener a la gente entusiasmada, al mismo tiempo establecer expectativas realistas sobre el tiempo de disponibilidad.

El camino de cada organización será único, hay patrones comunes, enfoques, y las mejores prácticas que se pueden implementar para agilizar el proceso. 
- Tener una clara comprensión de su actual costo relacionado es un primer paso importante. Esto proporciona la línea de base para definir el modelo de migración que ofrecerá un costo óptimo con la eficiencia requerida.

- Definir su enfoque de cloud computing desde el caso de negocio a la estrategia de gestión del cambio a la tecnología.

- Construir una base sólida para sus cargas de trabajo de la empresa en AWS, evaluando validar su cartera de aplicaciones, e integrar su tecnología con soluciones basadas en los servicios cloud de AWS.

- Diseñar y optimizar las aplicaciones empresariales para que sean conscientes de la ventaja directa de los beneficios de los servicios de AWS.

- Satisfacer sus requisitos de cumplimiento interno y externo desarrollando y la implementación de políticas de seguridad automatizadas y controles basados en probados, diseños validados.

\section{Buenas Prácticas}

De acuerdo a lo anteriormente descrito, se puede concluir las siguientes buenas prácticas para la migración a la nube:

\section{Antes de la Migración}

- Determinar las cargas de trabajo, tanto de los servidores físicos como virtuales, dispositivos de almacenamiento, bases de datos, redes, etc. Para esta actividad se realiza el levantamiento de información del data center.

Recomendación: Se recomienda que los servidores se encuentren virtualizados para facilitar la migración.

Recomendación 2: Se recomienda migrar servidores con sistema operativo Windows o Linux y sus distribuciones (Tecnología Intel). Debido a que son los sistemas operativos más comunes.

- Tener una clara comprensión de su actual costo relacionado es un primer paso importante. Esto proporciona la línea de base para definir el modelo de migración que ofrecerá un costo óptimo con la eficiencia requerida. Para el cálculo se toma en cuenta los gastos siguientes: 
- Mantenimiento: Gastos de mantenimiento del ambiente

- Redes: Ancho de banda necesario

- Capacidad: Costo de sobreaprovisionar.

- Disponibilidad y energía: Existe algún plan de recuperación ante desastres, gastos por energía eléctrica, costos de sistemas de refrigeración y cualquier otro relacionado.

- Servidores: Promedio de utilización del servidor, costo de sobreaprovisionar.

- Espacio: Gasto por espacio utilizado.

- Evaluar las cargas de trabajo a fin de determinar qué cosas migrar y que cosas no. Para esta evaluación se deben tomar en cuenta los beneficios obtenidos al migrar dicha carga de trabajo a la nube y si es posible realizar la migración. Asignar alguno de los siguientes estados para las cargas de migración evaluadas:

- No se obtiene beneficio al migrar la carga de trabajo

- No es posible realizar la migración

- La carga de trabajo es candidata a la migración

- Las cargas candidatas a la migración deben ser analizadas a fin de elegir el modelo de entrega de servicios que mejor se adapte a la carga de trabajo a migrar. Recomendación: Para elegir el modelo de entrega de servicios se aconseja seguir la recomendación de IBM, sin embargo, no es restrictivo.

- Planear las migraciones por partes, a fin de minimizar el riesgo, comenzando por cargas de trabajo que no sean críticas, especialmente si es la primera vez que se migra una carga de trabajo de la organización a cloud.

- Realizar un benchmarking de proveedores de servicios cloud, a fin de evitar problemas como las barreras de salidas, o precios muy elevados. Los puntos para considerar en el benchmarking y su ponderación son los siguientes:

- Funcionalidad

- Soporte

- Fiabilidad

- Usabilidad 
- Mantenimiento

- Portabilidad

- Seguridad

- Satisfacción

- Precios

- Se debe tener preparado respaldos de información para las cargas a migrar en caso suceda algún imprevisto.

\section{Durante la Migración}

- En este punto se mueven las cargas de trabajo desde su origen hasta su destino (cloud), para realizar esta actividad se requiere de un seguimiento constante de las cargas de trabajo, cuyo fin es saber el estado de la migración.

- Se debe construir y probar una infraestructura de migración a la nube adaptable y resistente que resida dentro del centro de la nube híbrida.

- La migración se debe realizar de a pocos, comenzando por cargas de trabajo que no sean críticas para el negocio.

- La migración se debe dar en un horario que no afecte o afecte lo mínimo posible a las operaciones de la organización.

Recomendación: Se recomienda realizar la migración los fines de semana o en las madrugadas.

\section{Después de la Migración}

- Después de la migración, se deben hacer las pruebas de carga y estrés de los recursos, a fin de evaluar su funcionamiento en este nuevo entorno.

- Luego de realizar las pruebas, se debe realizar el pase a producción de las cargas de trabajo migradas.

Monitorizar todo, es decir contar con una estrategia integral de monitorización, garantiza que no se pase por alto ningún detalle a la hora de crear arquitecturas sólidas para las aplicaciones 
Esta sección consiste en definir las fases, pasos, lineamientos y procedimientos que una pyme debe de seguir para realizar la migración de los servidores hacia la nube. Para ello se define la manera en que debe de abordarse la gestión del proyecto de migración y cómo es que debe de ser implementado el modelo.

\section{Modelo de migración a la nube de los servidores de un data center}

A continuación, se presenta el modelo de migración a la nube de los servidores de un data center, el modelo consta de 3 partes: Input, solución y output. Todo el modelo esta soportado bajo la metodología ICMM que se explica en el punto 5.3. En la parte de input, entran los requerimientos y el estado actual de la organización.

- Repositorio cloud: Donde se almacena el archivo de los discos duros de la máquina virtual.

- Script: Comandos que permiten realizar diversas tareas en la plataforma cloud como puede ser crear roles, asignar privilegios, subir archivos, entre otros.

- Importer: Servicio de la plataforma cloud que permite importar el archivo de los discos duros en una imagen que contiene la máquina virtual.

- Instancia: Máquina virtual desplegada a partir de una imagen.

- Administración: Herramientas de monitoreo continuo que es brindada por la misma plataforma cloud y nos permite saber el estado actual de los servidores en la nube e informar si sucede algún evento inesperado.

Finalizando, nos encontramos con la parte de output, donde es el resultado del modelo. En esta parte tenemos los servidores migrados en cloud, y el documento de implementación; el cual se detalla en el punto 5.3.

En caso alguna organización no cumpla con las condiciones indicadas más adelante, no significa necesariamente que no posible migrar a la nube, sino que el presente modelo no abarca la migración de estos, o posiblemente se deba realizar pasos adicionales para poder encajar con las características del presente modelo. 
En primer lugar, los hipervisores de tipo 1 que se contemplan en el modelo son: XenServer, Microsoft Hyper-V y VMware ESXi; debido a que estos son los principales hipervisores utilizados en los data center tier I, según David Davis (vExpert, VCP/VCAP, CCIE) en el siguiente link: http://www.virtualizationsoftware.com/top-5-enterprise-type1-hypervisors/; para el caso de hipervisores de tipo 2, se puede incluir cualquiera de las opciones, siempre y cuando el formato del archivo de disco duro sea compatible con la plataforma cloud o la organización cuente con los medios para convertirlo a un formato compatible de la plataforma cloud seleccionada (ver la documentación de migración del propio proveedor).

El grafo del modelo a utilizar, el cual está basado en la metodología ICCM es el siguiente:

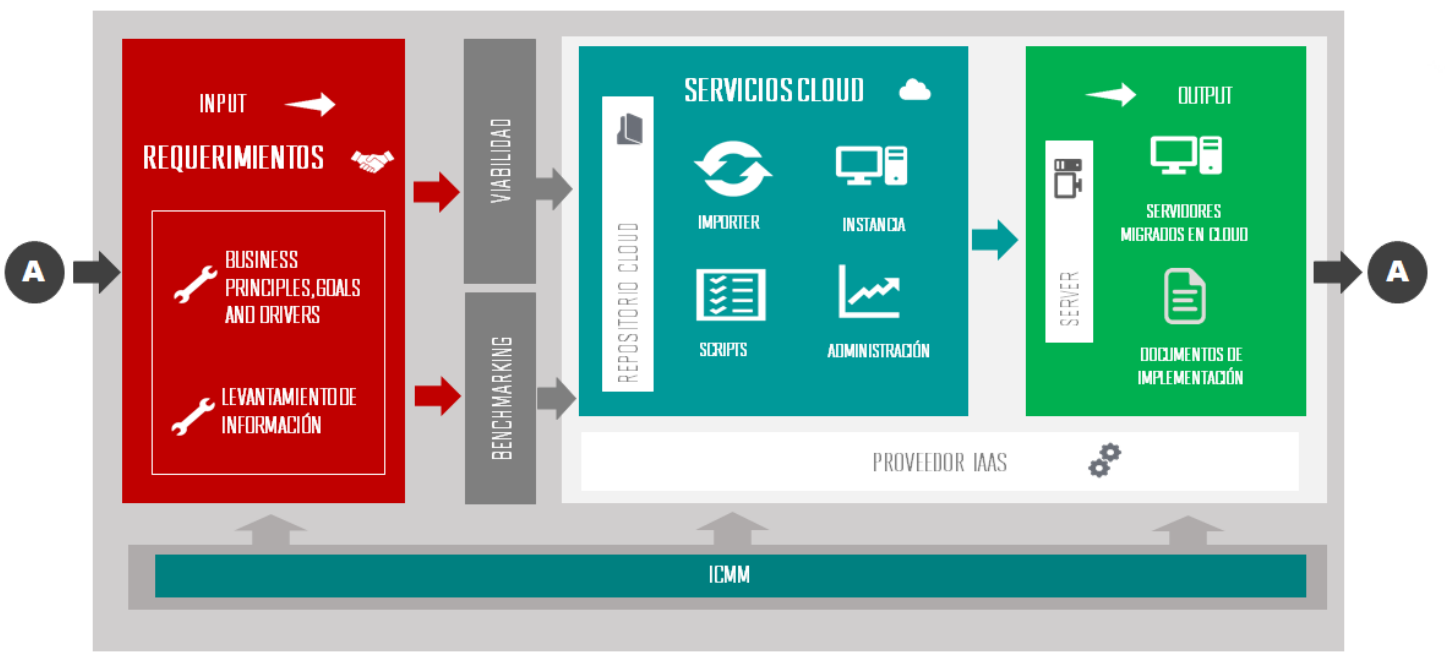

Ilustración 13: Grafo del modelo de migración a la nube de los servidores de un data center

Fuente: Elaboración Propia

Dentro del modelo, se ha de realizar la planificación del proyecto de migración a la nube, el cual nos permita medir el tiempo del proyecto, así como los recursos necesarios y la estimación de costos. Para esto también se debe seguir las buenas prácticas brindadas por el PMBOK, el libro del Project Management Institute (PMI).

1. Definir roles y responsabilidades: Es importante tener identificado todos los roles que participarán en el proyecto de implementación.

2. Definir la problemática: Es importante saber el porqué de la implementación, esta parte de la identificación de un problema en la empresa. 
3. Definir el alcance: En base a la problemática identificada, se define una solución que mitigue dicha falencia en la empresa.

4. Acondicionamiento de instalaciones: Es importante adecuar el espacio físico y tener listas las herramientas que son necesarias para el proyecto.

A continuación, se describe a detalle cada uno de los pasos mencionados anteriormente, así como quienes participan en cada uno de ellos y como contribuyen a una adecuada planificación de proyectos.

\section{Roles y responsabilidades}

Es importante conocer y mapear quienes serán los involucrados en el proyecto de migración a la nube. Saber con quién se debe trabajar y a quienes se necesita marcará el rumbo de todo proyecto. Para la ejecución del modelo propuesto, es necesario de tres roles importantes que contengan conocimiento técnico en específico para que nos apoyen y guíen durante el desarrollo del proyecto. En tal sentido, se ha definido los siguientes roles:

Analista de TI: Encargado de realizar los preparativos para la migración. Sus funciones principales son:

- Realizar el levantamiento e información de los activos de TI del cliente.

- Evaluar y seleccionar las cargas de trabajo a migrar.

- Convertir servidores físicos a virtuales que vayan a ser migrados.

Especialista Cloud: Experto en migración hacia Cloud. Sus principales funciones son:

- Conocer y entender las cargas de trabajo a migrar.

- Identificar recomendaciones y sugerencias para la migración a la nube.

- Realizar la migración de los servidores hacia la plataforma Cloud.

Usuario de Líder: Usuario de la organización con quien se realizan los acuerdos para la migración:

- Proporcionar el conocimiento sobre los activos de TI del negocio.

- Identificar y validar la propuesta del Especialista Cloud.

- Ser el canal de comunicación entre las necesidades de los demás usuarios involucrados con los otros miembros del equipo de trabajo. 


\section{Problemática de la empresa}

Roles involucrados: Analista de TI, Especialista Cloud y Usuario Líder.

Todo proyecto se inicia con el fin resolver un problema o cubrir una necesidad en una organización. En este sentido, es fundamental tener claro cuál es el problema que se desea solucionar en la organización y cuál es su impacto en la misma. Este problema es el crecimiento rápido y constante de una empresa, sea incompatible con la escalabilidad de la infraestructura del data center.

Por lo tantos se propone realizar tantas reuniones como sea necesaria entre el Usuario Líder, Analista de TI y Especialista Cloud.

\section{Alcance del proyecto}

\section{Roles involucrados: Usuario Líder y Especialista Cloud}

Una vez que se conoce la problemática, se debe definir una propuesta de solución que mitigue dicho problema. Se define qué será necesario y qué acciones se aplicarán para brindarle solución al problema.

En tal sentido, como parte del presente modelo, se debe tener una vista clara de la empresa, para lo cual se determina que cargas de trabajo que se desean migrar; sin embargo, la migración de las mismas depende de la posibilidad y beneficio de la migración que se realizará más adelante.

Es importante recordar que, el alcance del proyecto puede variar dependiendo de la problemática de la organización, al tamaño o a la complejidad de la migración de las cargas de trabajo a Cloud. Por ejemplo, si la empresa cliente no cuenta con los suficientes recursos para ejecutar aplicaciones, están siendo limitados de lo que pueden lograr hacer, y esto perjudicaría a su negocio. Otra casuística que se pueda dar es que la empresa cliente quiera en vez de reemplazar y reparar viejos servidores en sus instalaciones, puedan alojar estos en la nube e incrementar la seguridad e integridad de datos.

\section{Metodología}

Luego de analizar las metodologías brindadas por TOGAF y CCRA, y las buenas prácticas anteriormente analizadas en la literatura; elegimos los componentes más 
resaltantes y que permitirán agregar mayor valor al proceso de migración para crear una metodología que se enfoque en la migración de infraestructura de un data center a la nube, la cual llamaremos Infrastructure Cloud Migration Method, al cual se le llamará a partir de este momento como ICCM.

Primero comenzaremos detallando lo que se absorbió de la metodología brindada por TOGAF:

De la fase preliminar, se eligió el siguiente entregable:

- Principios, objetivos y motivadores de negocio: Se deben conocer los objetivos de la organización, de esta manera poder alinearnos estos en el proyecto de migración.

De la fase B (Arquitectura de Negocio), se eligieron los siguientes entregables:

- Architecture Requirements Specification: Nos permite identificar los requerimientos del negocio, al cual va a alinear el proyecto.

De las fases C y D (Arquitectura de sistemas de información y tecnologías), se eligió lo siguientes:

- Levantamiento de las aplicaciones e infraestructura existente: Permite conocer los activos actuales y que activo podrían migrarse a la nube.

En Segundo lugar, se detalla lo absorbido por la metodología brindada por CCRA:

De la fase Understanding se eligió los siguientes entregables:

- Business Drivers: Nos permite entender los objetivos del negocio.

- Levantamiento de Información: Permite conocer los activos actuales de la organización.

De la fase Explore, se eligió los siguientes entregables:

- Non-Functional Requirements: Nos permite determinar los requerimientos no funcionales esperados por la organización.

De las buenas prácticas de la literatura, se definieron los siguientes entregables: 
- Levantamiento de información: Se debe conocer los activos de TI que se poseen, para poder tomar decisiones en torno a estos.

- Levantamiento de requerimientos: Se deben identificar los requerimientos, tanto los funcionales como los no funcionales.

- Benchmarking de Proveedor: Se debe evaluar a los proveedores para poder elegir la mejor opción de acuerdo a los requerimientos.

- Evaluación de viabilidad: Se debe evaluar que tan viable es la migración de acuerdo a los criterios económicos, técnicos y operativos, en base a esto se deciden que cargas se migrarán y cuáles no.

- Documento de implementación: Se debe documentar como se realizó la migración y los datos relevantes de cada servidor migrado.

Después de los datos recopilados, se procedió a conectar lo recopilado y se creó la siguiente metodología, la cual se divide en 4 fases:

Entendimiento: En esta fase se busca entender al cliente, tanto sus objetivos como sus activos de TI, en este sentido se realizan los siguientes entregables:

- Principios, objetivos y motivadores de negocio: Se colocan los objetivos de la organización y que se espera conseguir, lo que permite alinear al proyecto de migración con estos mencionados.

- Levantamiento de información: Se levanta la información de la infraestructura del cliente, esto permite saber que se tiene y que se hará con estos.

Exploración: En esta fase se exploran a los clientes y sus necesidades, para lo cual se realiza el siguiente entregable.

Especificación de requerimientos: Se detallan los requerimientos del cliente, tanto funcionales como no funcionales.

Evaluación: En esta fase se evalúa lo que se migrará y a donde se migrará, para lo cual se utiliza los siguientes entregables: 
- Benchmarking: Se realiza un análisis comparativo entre los proveedores de plataformas cloud, y se elige la mejor opción de acuerdo a la necesidad.

- Estudio de viabilidad: Se analiza la viabilidad de la migración de cada carga y del alcance del proyecto, tanto económica, técnica y operativa.

Migración: Se realiza la migración de los servidores elegidos, para esta fase se realiza el siguiente entregable:

- Documento de implementación: Se explica que pasos se realizó para la migración.

La metodología presentada soporta el modelo de migración a la nube de los servidores de un data center.

Ahora pasa, se detalla cómo proceder en cada fase de la metodología y las plantillas a utilizar:

\section{FASE I: Entendimiento}

En esta etapa se da el entendimiento del cliente, para de esa manera poder plantear la solución, por lo tanto, para esta parte se deben desarrollar los siguientes entregables:

- Principios, objetivos y motivadores de negocio: Son los manejadores del negocio claves para el proyecto, es decir los principales puntos que nos permite migrar a cloud, estos están impulsados por el negocio.

- Levantamiento de información: Es el estado actual de ambiente de TI, es decir un levantamiento de información de los activos de TI.

\section{Principios, objetivos y motivadores de negocio}

Roles involucrados: Analista de TI, Usuario Líder

En este punto se realizan lo Principios, objetivos y motivadores de negocio, para lo cual se hace uso de la plantilla "Principios, objetivos y motivadores de negocio", esta contiene lo siguiente:

1. Propósito del Documento: Se define por qué motivo se realiza el documento.

2. Principios de Negocio: Se describen los principios del negocio

3. Objetivos de Negocio: Se colocan las metas del negocio con el proyecto 
4. Motivadores de Negocio: Se colocan los objetivos de negocio a los cuales alinearemos el proyecto.

Se puede revisar la plantilla del documento en el anexo 1.

\section{Levantamiento de Información}

Roles involucrados: Usuario Líder, Analista de TI

Para el levantamiento de información de activos de TI se utiliza la plantilla "Levantamiento de Información", en la cual se recopila la información de las cargas de trabajo (Hardware, Base de datos, Aplicaciones, Sistemas Operativo) y de los procesos y roles de TI.

El documento está compuesto por los siguientes puntos:

1. Inventario de servidores de la empresa: En este punto se colocan los siguientes atributos:

- Servidor físico: Se indica el nombre del servidor físico.

- Características: Se indican las características técnicas del servidor físico.

- Servicios: Se indican los servicios que se incluyen en el servidor físico.

- Descripción: Se brinda una descripción del servidor físico

- Servidor virtual: Se indican los servidores virtuales del servidor físico, en caso existan.

- Características servidor virtual: Se indican las características técnicas del servidor virtual.

- Involucrados: Se indican los involucrados con el servidor indicado.

2. Diagrama de Infraestructura: Se indican la distribución de los servidores de la organización

- Infraestructura con software base: Detalla infraestructura física con sw base actual de la organización.

- Virtualización: Detallar la virtualización actual del usuario

- Servicios

3. Configuración de Firewall: Detallar configuración del firewall actual

Se puede revisar la plantilla del documento en el anexo 2. 


\section{FASE II: Exploración}

Posteriormente, se debe identificar y definir los requerimientos, con esta base se puede decidir qué es lo que se realizará durante el proyecto.

Para esta etapa se ha de desarrollar el siguiente entregable:

- Requerimientos funcionales y no funcionales: Se definen los requerimientos funcionales y no funcionales del proyecto a realizar.

\section{Especificación de Requerimientos}

Roles involucrados: Usuario Líder y Especialista Cloud

En este paso se busca identificar los requerimientos de la migración, tanto funcionales como no funcionales, para esto se debe utilizar la plantilla de "Especificación de Requerimientos" adjunta al modelo. Los requerimientos deben ser coordinados entre el Usuario Líder y el Especialista Cloud; y por lo tanto debe estar firmado por ambas partes.

El documento consta de los siguientes puntos:

1. Antecedentes: En este punto se debe detallar cual es el estado actual de la organización.

2. Objetivo del requerimiento: Se deben colocar los objetivos que realizarán con el proyecto, es decir, que es lo que se espera conseguir con el proyecto.

3. Descripción del requerimiento: La descripción del requerimiento consta de los siguientes eventos:

- Alcance funcional: En este punto se debe indicar cuál será el alcance del proyecto de migración a realizar.

- Requerimientos funcionales: En este punto se colocan los requerimientos funcionales del proyecto de migración.

- Requerimientos no funcionales: En este punto se colocan los requerimientos no funcionales del proyecto de migración.

- Exclusiones (a ser llenado por el especialista): Este punto debe ser llenado por el Especialista Cloud, aquí se indican cuáles serán las exclusiones del proyecto.

4. Alcance técnico: Se indican los recursos necesarios para realizar el requerimiento.

5. Requerimientos No Funcionales: El usuario Líder indica cuáles serán sus requerimientos no funcionales para la migración. 
6. Consideraciones: Se indican las consideraciones del requerimiento.

Se puede revisar la plantilla del documento en el anexo 3.

\section{FASE III: Evaluación}

En esta fase se evalúan tanto el proveedor que se elegirá como las cargas que se migrarán, en esta fase se realizan los siguientes entregables:

- Benchmarking: comparación entre proveedores de IaaS para tomar la mejor decisión.

- Estudio de viabilidad: Se realiza el estudio de viabilidad de la migración, tanto económica, técnica y operativa.

\section{Benchmarking}

Roles involucrados: Usuario Líder y Especialista Cloud

Para la selección de la Plataforma Cloud, se debe realizar un benchmarking de las distintas plataformas, el cual para establecer el Modelo de Calidad a utilizar dentro de la evaluación se considera la ISO 2500, conocido como Square (System and Software Quality Requirements and Evaluation). Para ello se utiliza plantilla de "Benchmarking" adjunta al modelo. Este documento (Benchmarking) cuenta de los siguientes puntos:

1. Descripción del Activo Preexistente: En este punto se detalla el objetivo por el cual se realiza el benchmarking

2. Alternativas a considerar: En este punto se colocan las alternativas a comparar para el documento.

3. Análisis comparativo: En este punto se indican los criterios que se utilizan para evaluar las alternativas y el porqué, además de indicar las métricas

4. Propósito de evaluación: Se indica el propósito de valuación de benchmarking.

- Modelo de Calidad: Se indica bajo qué criterios se evalúa la herramienta/plataforma

- Métricas: En este punto se colocan los criterios de calificación

- Evaluación técnica: En base al modelo de calidad para la calidad externa e interna se establecieron los atributos a valorar a evaluar, en este caso son los siguientes:

1. Funcionalidad: Cumplimiento de las funciones principales $100 \%$ de administrar servidores, memoria y redes. 
2. Fiabilidad: Capacidad de mantener un nivel especificado de análisis y solución, en caso de errores.

3. Usabilidad: Facilidad y rapidez de comprensión a nivel usuario y técnico.

4. Eficiencia: Alto rendimiento y consumo mínimo de recursos

5. Mantenimiento: Facilidad de actualización y soporte técnico local.

6. Portabilidad: Adaptabilidad a distintos entornos de software y facilidad de instalación.

7. Eficacia: Capacidad de alcanzar metas operativas al $100 \%$ al momento de uso de las funciones de administración de servidores, memoria y redes.

8. Seguridad: Capacidad de ser confiable y seguro para la información que se administre en dicha herramienta.

9. Satisfacción: Capacidad de cubrir los requerimientos con total conformidad de usuario.

10. Productividad: No debe consumir muchos recursos de memoria y procesador en los equipos donde se haya instalado.

5. Conclusiones: En este punto se coloca el resultado de la comparación y algún comentario que pudiese ser interesante al momento de tomar una decisión de la plataforma.

La organización que implemente el modelo es libre de elegir la plataforma Cloud que considere apropiada y se ajuste de mejor manera a sus necesidades y expectativas, sin embargo, se recomienda el uso de Amazon Web Services (AWS) basándose en el resultado de nuestro análisis:

Tabla 3: Resultados del Benchmarking

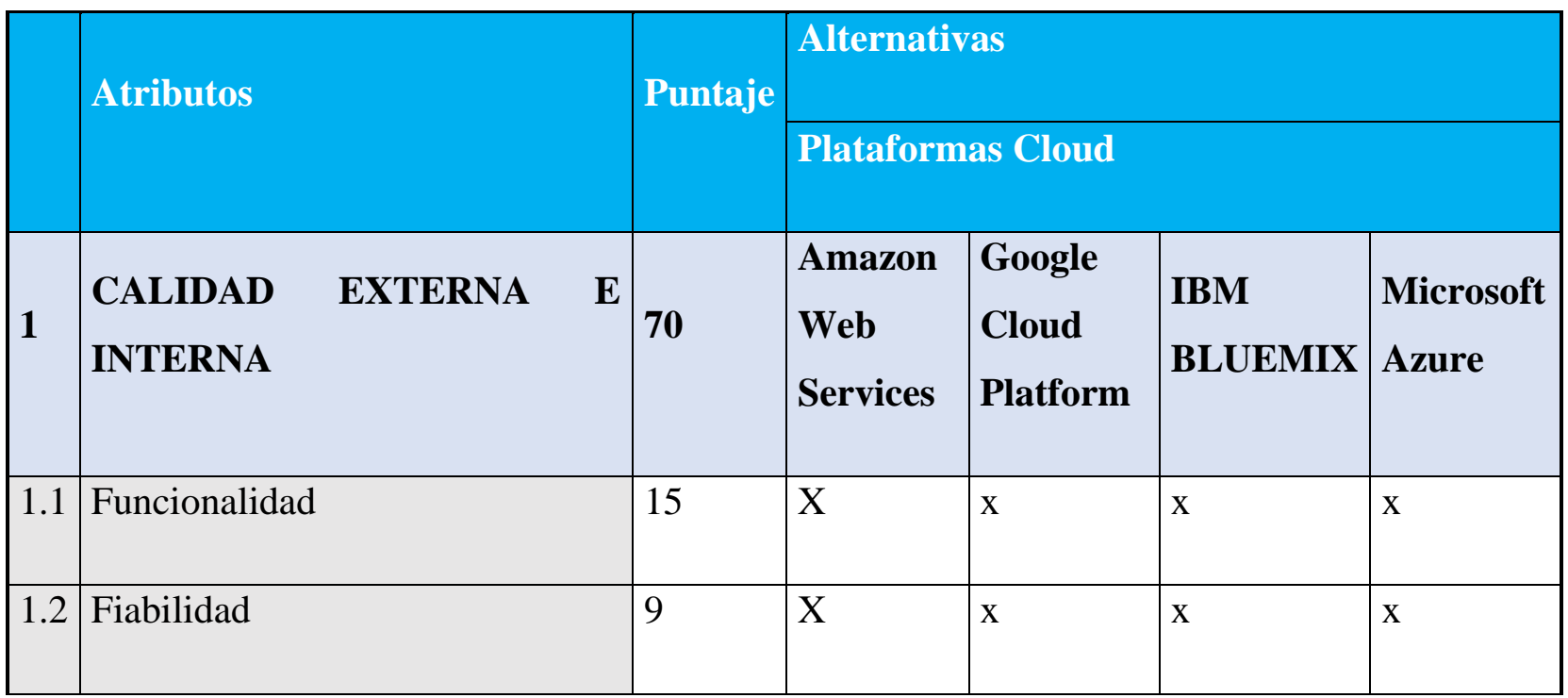




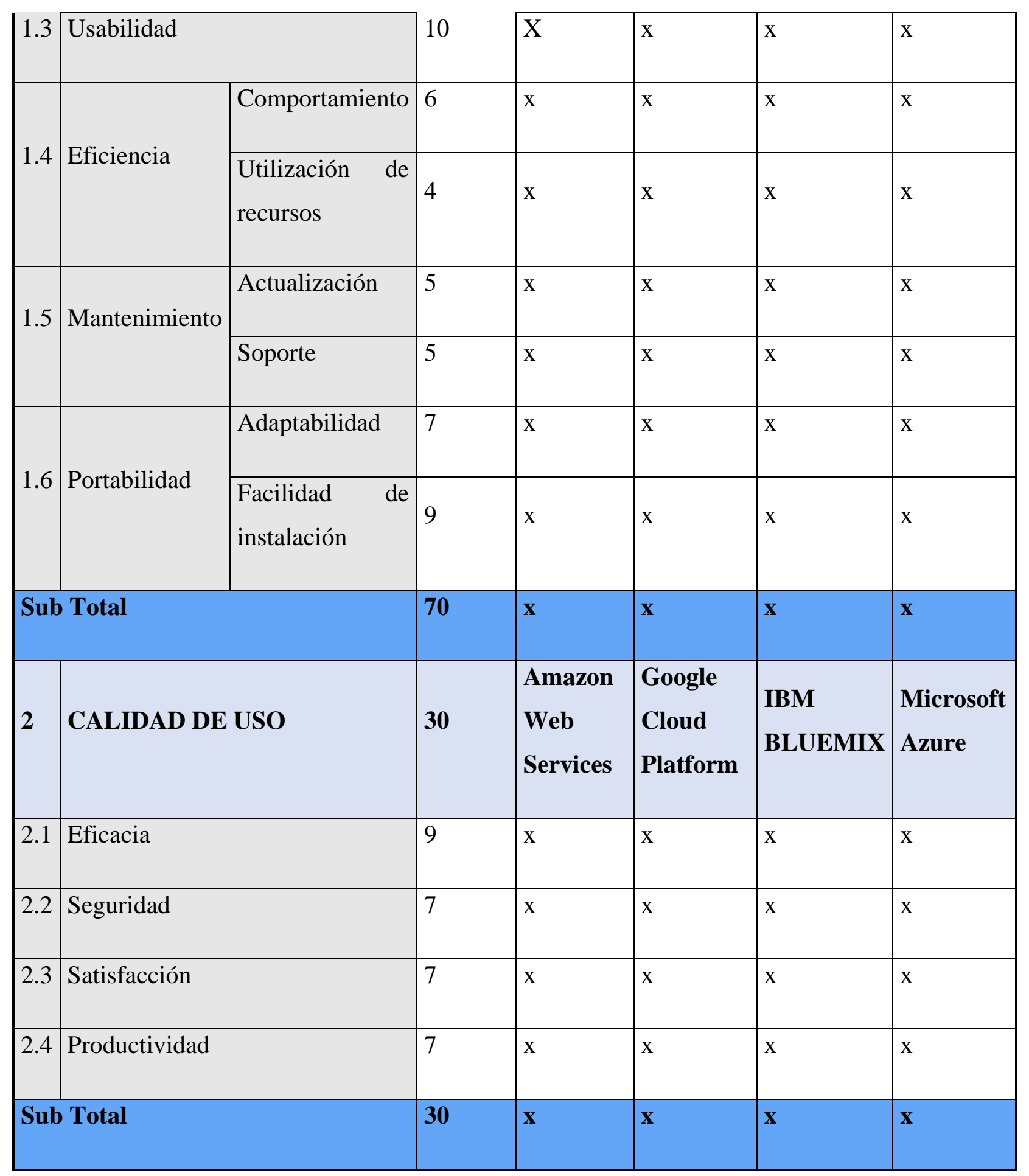

Fuente: Elaboración Propia

Se puede revisar la plantilla del documento en el anexo 4 .

\section{Estudio de viabilidad}

Roles involucrados: Analista de TI, Especialista Cloud 
Se requiere evaluar los 3 puntos antes mencionados: económica, técnica y operativa, para ello se usa la plantilla "Estudio de viabilidad". Esta plantilla contiene los siguientes puntos:

1. Estudio de viabilidad técnica: En la literatura, se evalúa y selecciona de las cargas de trabajo. Un ejemplo de ello es el trabajo de J. Octavio Gutiérrez García, quien en su trabajo evalúa estas cargas para agruparlas. ${ }^{39}$ para esta actividad se puede hacer uso de la herramienta "Viabilidad_Técnica.xlsx", en la cual se evalúan las cargas de trabajo, la intención es etiquetar cada carga de trabajo con alguno de los siguientes estados:

- Migración no factible: Para este caso se debe tener en cuenta la posibilidad de migrar a la nube bajo este modelo, esto puede deberse a los siguientes motivos:

1. El servidor posee un sistema operativo que no corresponde a la tecnología Intel (que es la que se contempla en este modelo).

2. Componentes no disponibles en Cloud.

3. Procesos y transacciones demasiado complejas.

4. Cargas de trabajo relacionados a alguna regulación sensible.

5. Cargas de trabajo altamente personalizados.

6. Software de terceros no virtualizado.

7. Hipervisores no contemplados por el modelo.

- Migración muy Compleja: En este caso se debe tener en cuenta que la migración es posible, sin embargo, debido a los pasos adicionales que se deben realizar, es necesario evaluar bien si se desea migrar, pues es posible que el beneficio no sea el esperado o sea negativo por los pasos adicionales realizar.

- Migración compleja pero factible: Es decir que las cargas de trabajo son posibles de migrar, pero requieren algunos pasos adicionales, a diferencia de la etiqueta anterior, esta es más probable de brindar un beneficio positivo.

- Migración factible: Indica que a migración no es complicada de realizar y por lo tanto es candidata para migrar.

8. Estudio de viabilidad económica: Se debe evaluar que económicamente el proyecto trae beneficios a la organización, para esto se puede hacer uso de la herramienta "Viabilidad_Ecnónomica.xlsx", en la que se colocan costos del proyecto y los beneficios que obtendrán.

${ }^{39}$ J. Octavio Gutierrez Garcia 
9. Estudio de viabilidad operativa: Se evalúa si las operaciones luego de realizar el proyecto podrán realizarse correctamente y sin impactar al negocio de forma negativa. 10.

Este entregable puede brindar como resultado que no se realizará ninguna migración debido a que no genera beneficios o que no es posible debido a los puntos antes señalados, lo que significa que el proyecto no se llegará a concretar.

Se puede revisar la plantilla del documento y herramientas en el anexo 5

\section{FASE IV: Migración}

Esta fase consta de la realización de la migración de los servidores, es decir desde la virtualización de servidores físicos en caso se requiera, hasta la puesta en marcha, monitoreo y administración de la Plataforma Cloud.

\section{Documento de Implementación}

Roles involucrados: Especialista Cloud

En esto documento se debe narrar como se realizó la implementación de la migración, de esta manera se deja documentado como se realizó el proyecto que podría ser requerido para algún cambio necesario en el futuro. Para esto se debe hacer uso de la plantilla "Documento de implementación”, la cual contiene los siguientes puntos:

1. Propósito del documento: Se debe detallar el motivo por el cual se desarrolla el presente entregable.

2. Proceso de migración: Se detalla paso a paso el proceso de la migración.

3. Conclusiones: Se explican los resultados finales de la implementación.

A continuación, se muestra como migrar haciendo uso de la plataforma Amazon Web Services:

En primer lugar, en caso de contar con servidores físicos a migrar, se debe utilizar un conversor de máquina física a virtual, para esto se pueden utilizar diferentes herramientas, a continuación, se colocan algunas opciones, sin embargo, cada quien es libre de elegir la herramienta de su preferencia:

\section{- Disk2VHD}


- Microsoft Virtual Machine Converter

- VMWare vCenter Converter

- Paragon Hard Disk Manager

Antes de realizar la migración se debe conseguir el espacio solicitado en la plataforma cloud, una vez cuente con el espacio requerido, se procede moviendo las cargas de trabajo del punto de origen (Data Center on-premise) hacia el punto de destino (Plataforma Cloud). Para efectos del caso, se debe tener en cuenta que este proceso depende de la red (velocidad, puertos bloqueados, conexiones vpn, etc.).

Se recomienda que la primera migración no sea una carga de trabajo crítica para el negocio. También se recomienda que la migración se de en un horario en el que no genere impacto o el mínimo impacto posible a la organización, el cual generalmente es en fines de semana o madrugadas.

Se puede revisar la plantilla del documento en el anexo 6.

La migración de las máquinas virtuales varía dependiendo de la Plataforma Cloud, para este caso se utilizó AWS y se listan los pasos a continuación:

\section{Configurar EC2 API Tools Client}

Colocar la carpeta EC2-api-tools-1.7.5.1 en el disco local C

$\leftarrow \rightarrow \sim \uparrow:$, Este equipo > Windows (C:)

\begin{tabular}{|c|c|c|c|c|c|}
\hline \multirow{2}{*}{\multicolumn{2}{|c|}{ Acceso rápido }} & Nombre & Fecha de modifica... & Tipo & Tamaño \\
\hline & & AMD & 27/05/2017 11:19 a... & Carpeta de archivos & \\
\hline Escritorio & $\star$ & Archivos de programa $(x 86)$ & 2/08/2017 8:51 p. m. & Carpeta de archivos & \\
\hline$\checkmark$ Descargas & * & inetpub & $27 / 05 / 201712: 03 \ldots$ & Carpeta de archivos & \\
\hline 图 Documentos & * & Intel & $23 / 11 / 201612: 51 \ldots$ & Carpeta de archivos & \\
\hline$\triangle$ Imágenes & $\star$ & PerfLogs & 18/03/2017 4:03 p.... & Carpeta de archivos & \\
\hline$\checkmark$ 2017-01 & $\star$ & Program Files & 2/09/2017 11:53 a. ... & Carpeta de archivos & \\
\hline Actas & & sWSetup & 3/08/2017 9:57 p. m. & Carpeta de archivos & \\
\hline Modelo Proyecto & & Usuarios & 27/05/2017 11:25 а.... & Carpeta de archivos & \\
\hline & & Windows & 26/08/2017 11:53 а... & Carpeta de archivos & \\
\hline Paper & & $\square$ listener.ora & 25/06/2017 1:26 p.... & Archivo ORA & $3 \mathrm{~KB}$ \\
\hline$\square$ IP2 & & 望 windows-version.txt & 4/07/2017 8:49 p. m. & Documento de tex... & $1 \mathrm{~KB}$ \\
\hline OneDrive & & ec2-api-tools-1.7.5.1 & 7/09/2015 7:56 a. m. & Carpeta de archivos & \\
\hline
\end{tabular}

Descargar e instalar java desde la siguiente dirección en caso no lo tenga: https://java.com/es/download/win10.jsp 


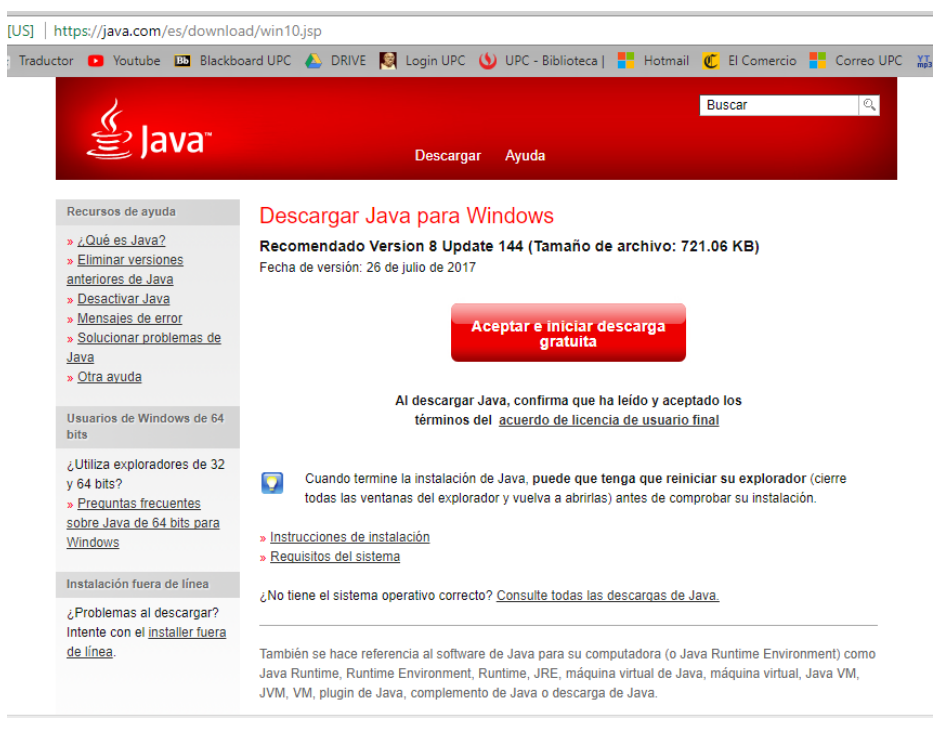

Ingresar al explorador de windows

Realizar clic derecho en Equipo y entrar a las propuedades del equipo.

\section{Acceder Configuración avanza da del sistema -> Variables de Entorno}

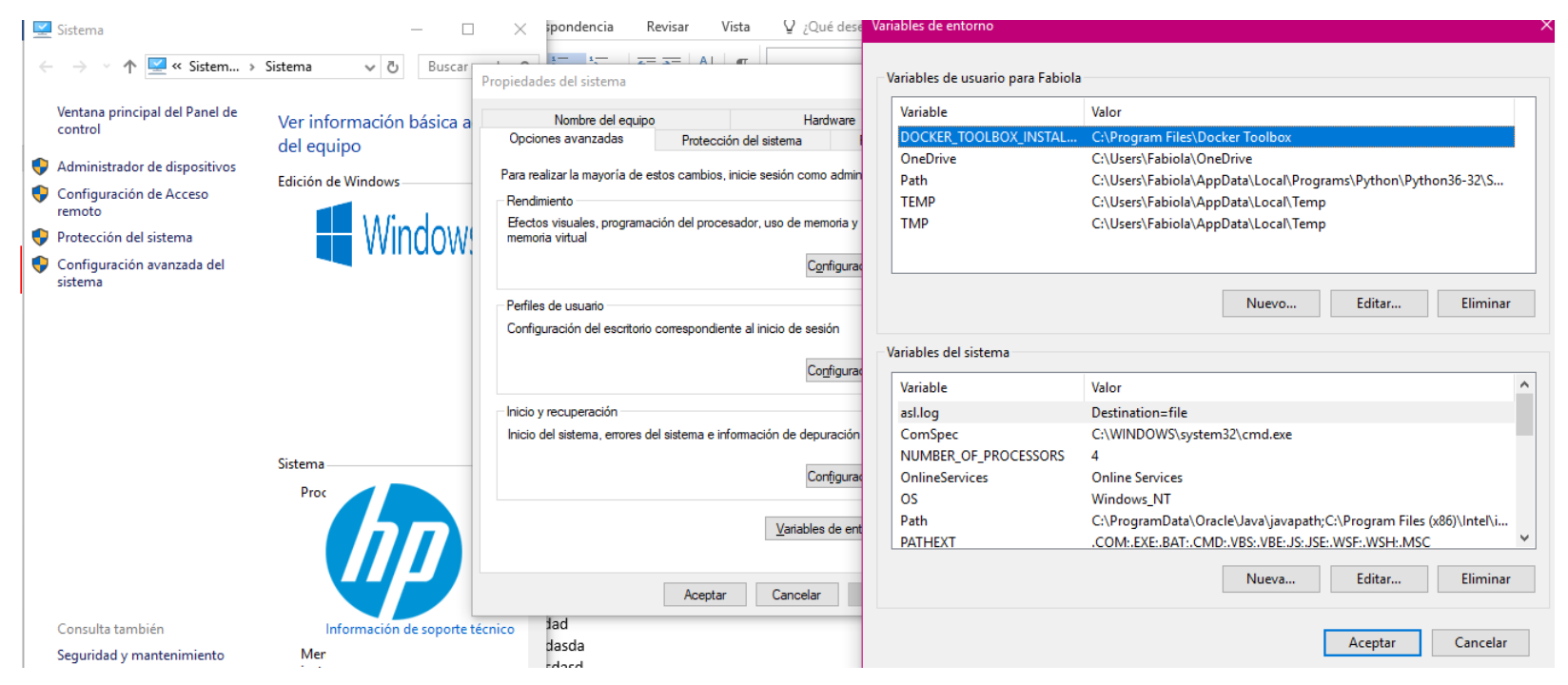

Ingresar uan nueva variable de entorno para el usuario, llamarlo JAVA_HOME, y colocar como valor la ruta de instalación de java hasta la carpeta llamada jre[versión java] y aceptar. 


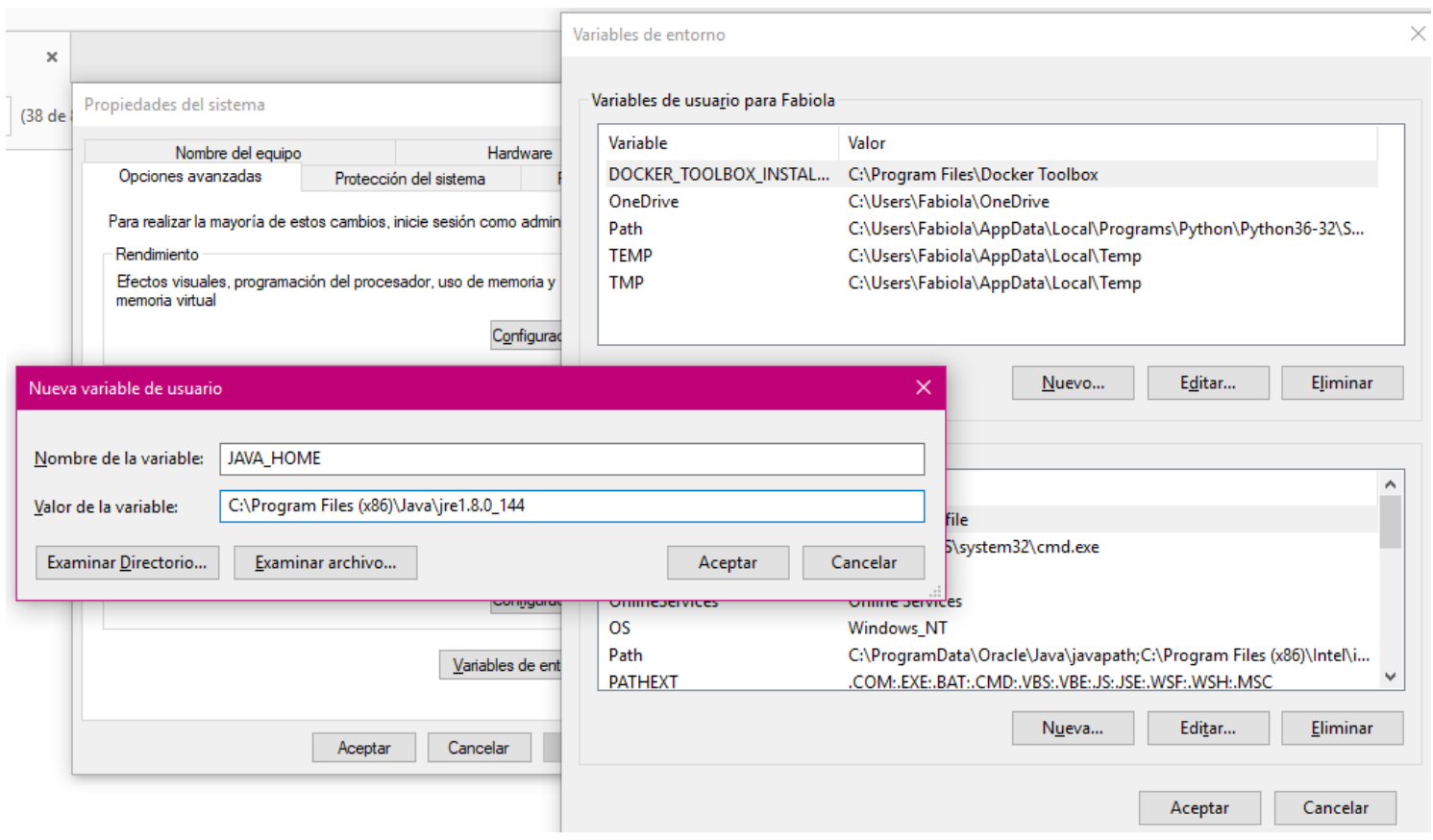

Agregar a la variable de entorono para usuario Path el valor que se muestra en la imagen:

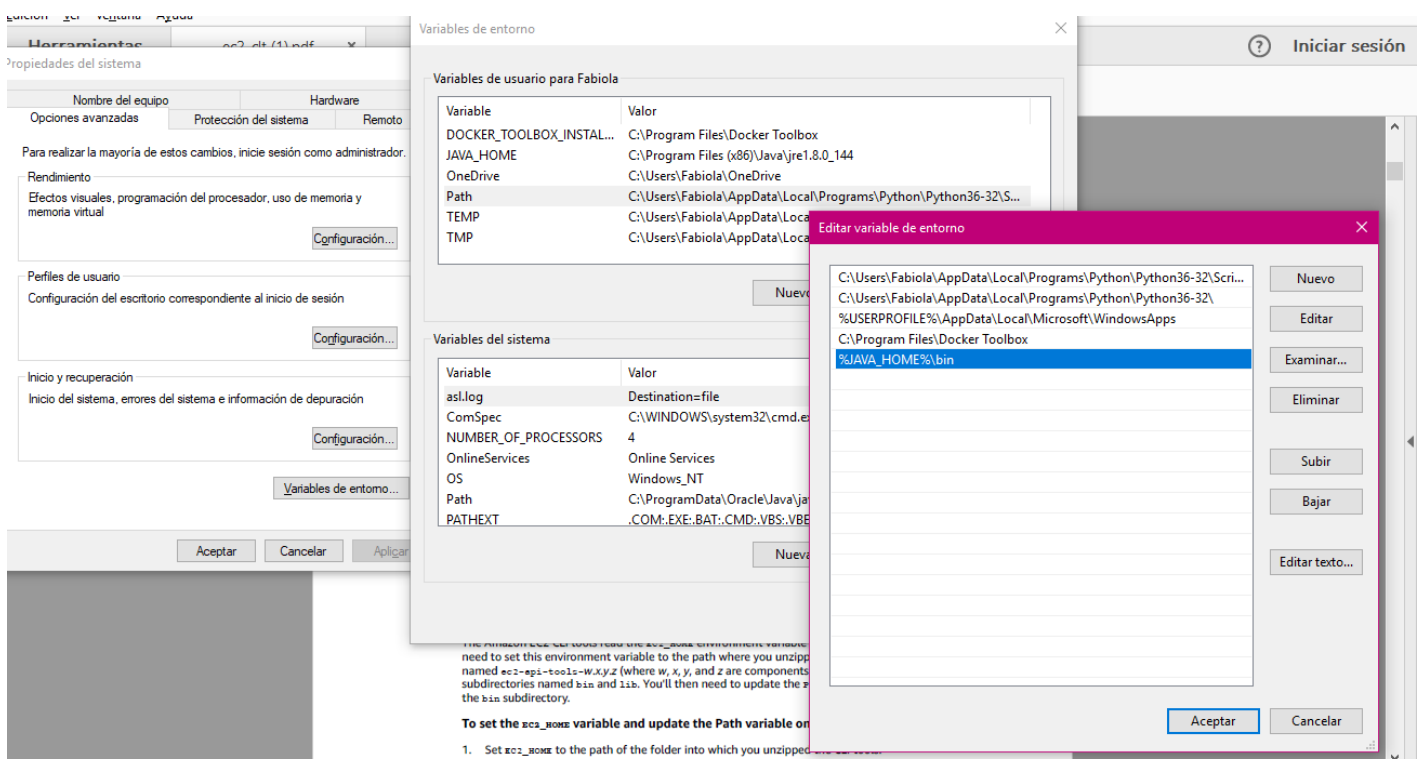

Agregar la variable de entorno para usuario EC2_HOME colocando como valor la ruta de la carpeta EC2-api-tools-1.7.5.1. 


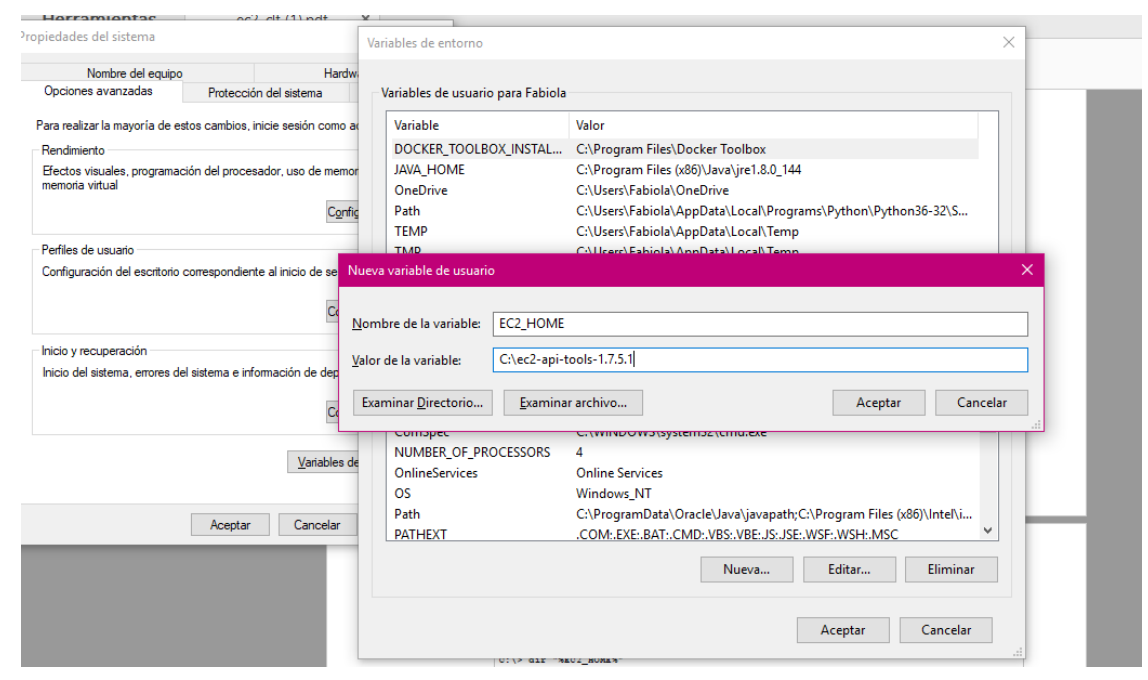

Actualizar la variable Path agregando el valor \%EC2_HOME\%lbin

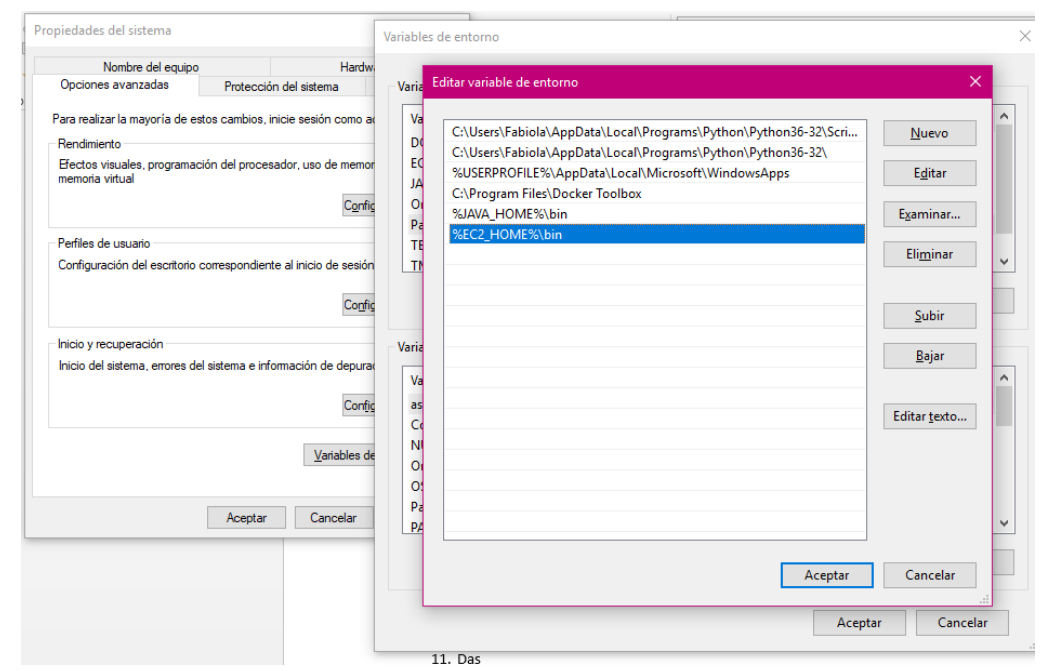

\section{Configurar credenciales de acceso}

Abrir cmd y escribir el comando aws configure

Indicar sus credenciales, region por defecto y formato de salida. 


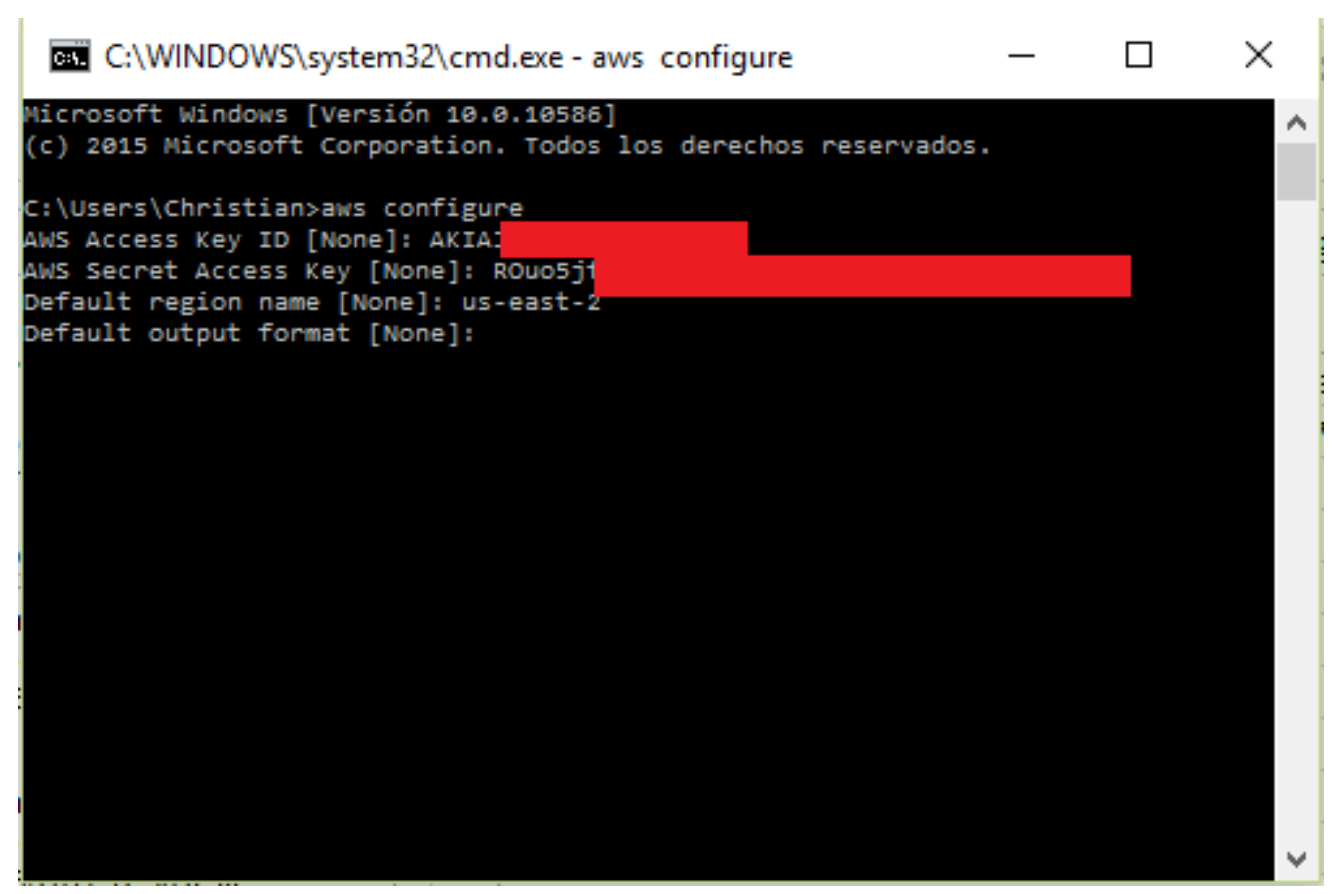

\section{Migrar Maquina Virtual a AWS}

Crear un bucket para subir la máquina virtual en caso no exista, hacer clic en crear nuevo y colocarle un nombre (no se puede repetir etre todos los bbuckets globales).

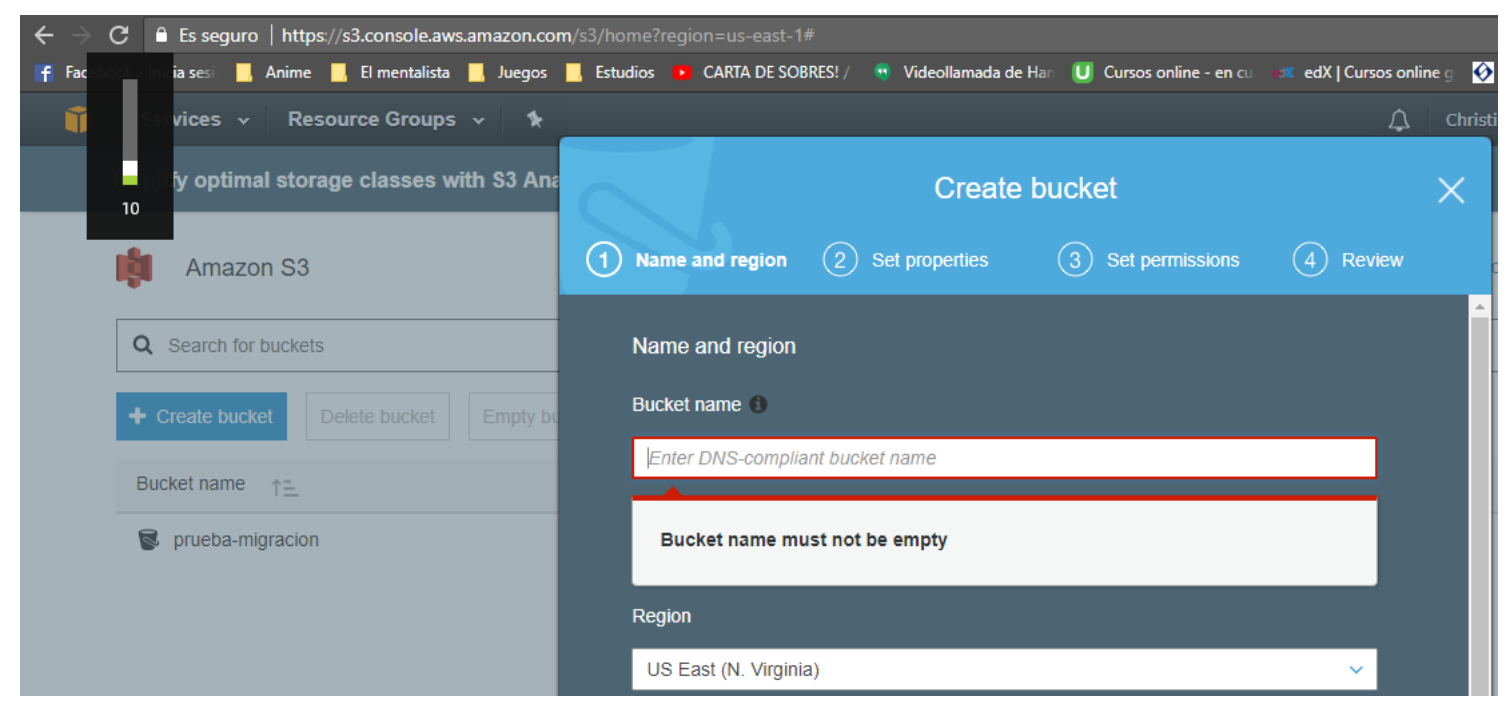

Crear el archivo trust-policy.json con la siguiente estructura:

"Version": "2012-10-17", 


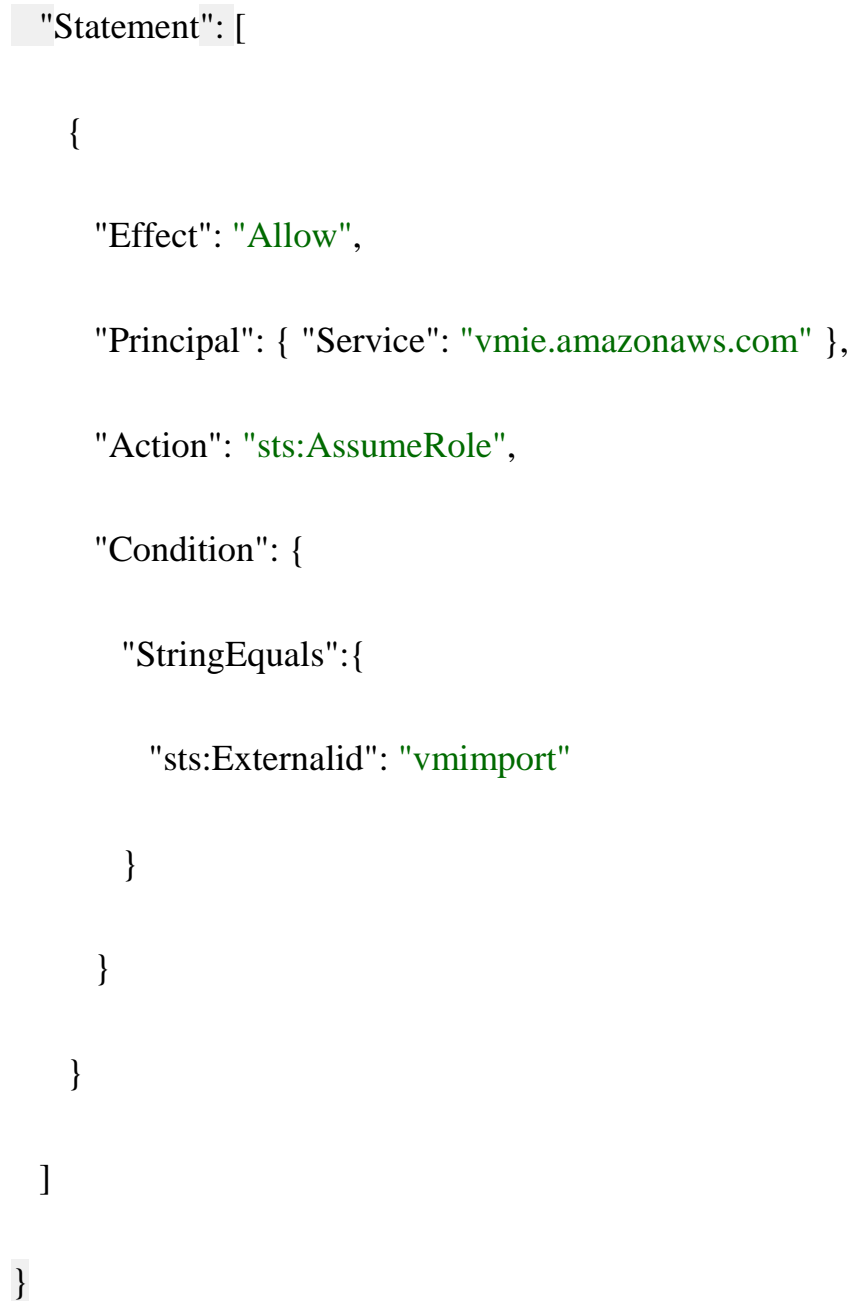

Invocar el siguiente comando en el CLI para crera el rol vmimport: aws iam createrole --role-name vmimport --assume-role-policy-document [file://trust-policy.json], el cual devolverá el siguiente mensaje de creación de rol. 


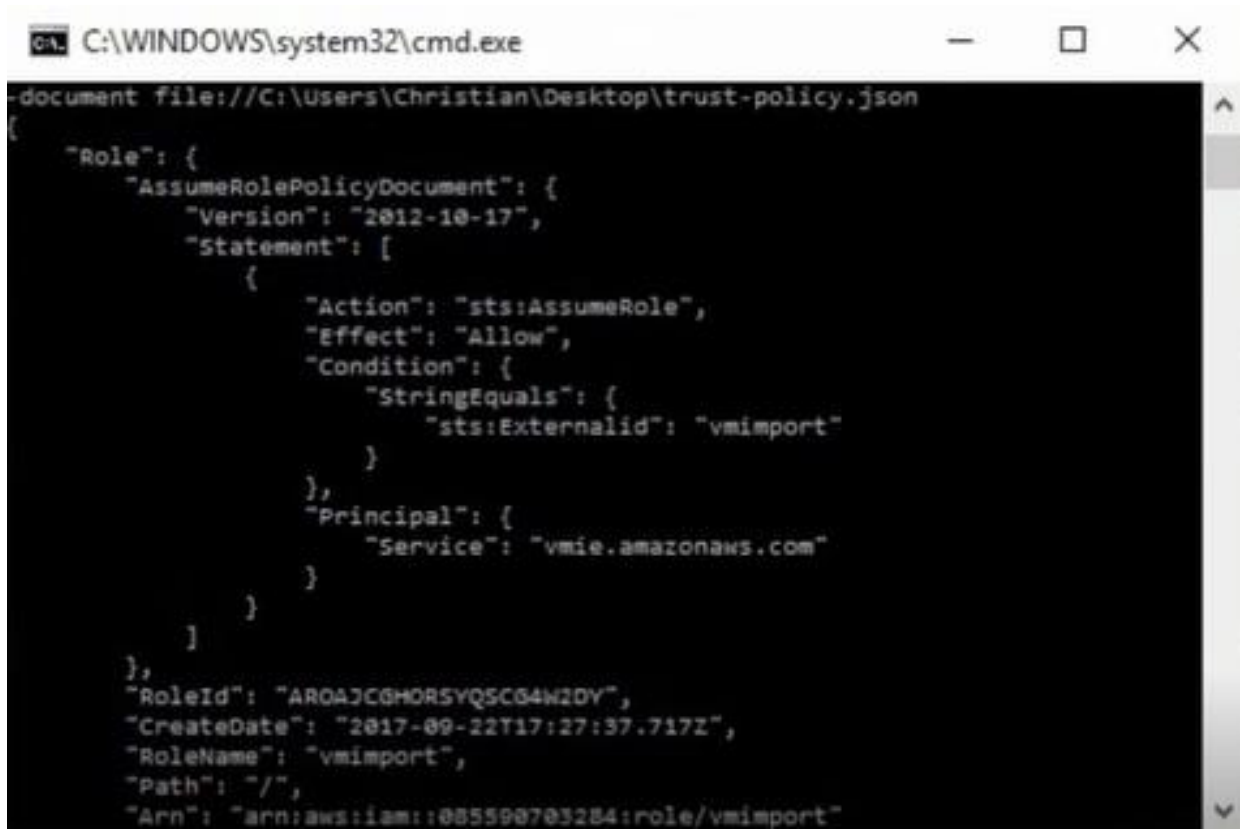

Crear el archivo role-policy.json con la siguiente estrictura, donde disk-image-file-bucket representa el bucket donde se subirá el archivo de la vm:

\{

"Version": "2012-10-17",

"Statement": [

\{

"Effect": "Allow",

"Action": [

"s3:ListBucket",

"s3:GetBucketLocation"

],

"Resource": [

"arn:aws:s3:::disk-image-file-bucket"

] 
\},

\{

"Effect": "Allow",

"Action": [

"s3:GetObject"

],

"Resource": [

"arn:aws:s3:::disk-image-file-bucket/*"

]

\},

\{

"Effect": "Allow",

"Action":[

"ec2:ModifySnapshotAttribute",

"ec2:CopySnapshot",

"ec2:RegisterImage",

"ec2:Describe*"

],

"Resource": "*"

\}

]

\} 
Invocar el siguiente comando en el CLI: aws iam put-role-policy --role-name vmimport --policy-name vmimport --policy-document [file://role-policy.json], el cual asignara las politicas de migración al rol antes creado.

Subir el archivo .VHD, .VMDK o .VHI a Amazon S3 (Storage) utilzando el siguiente

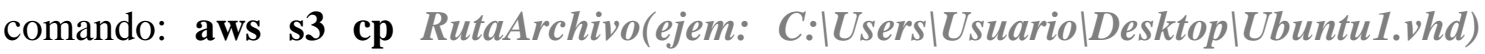
s3://Bucket y observará el progreso de la acción:

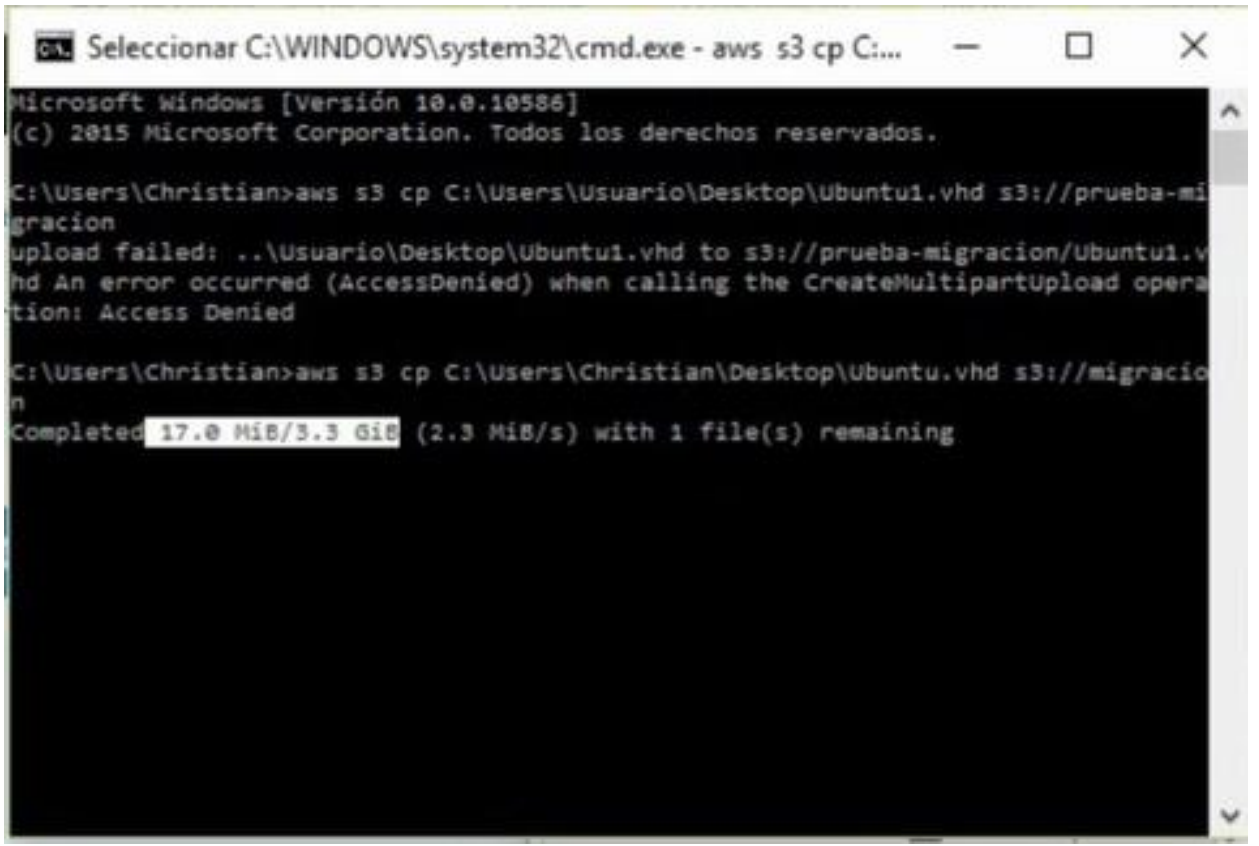

Crear el arhivo containers.json con la siguiente estructura.

"Description": "[Colocar descripción]",

"Format": "[VHD, VMDK, VHI]",

"UserBucket": \{ 


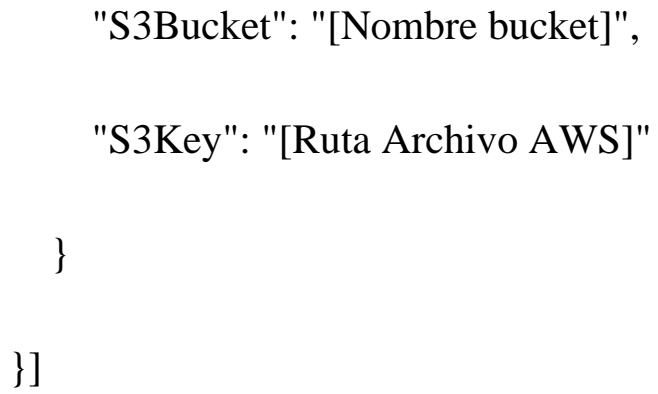

Ejecutar el comando de importación de maquina virtual: aws ec2 import-image -description "[descripción]" --license-type [Tipo de licencia]--disk-containers file://[RutaArchivoContainers], se pocederá a importar la vm.

En caso desee revisar el estado ejecure el siguiente comando: aws ec2 describeimport-image-tasks --import-task-ids import-ami-[id]

Cuado se complete, se generará como una AMI (Amazon Machine Image) propia.

\section{Lanzamiento de la VM}

Ingresar a Amazon EC2 e ir a la seción Launch Instance.

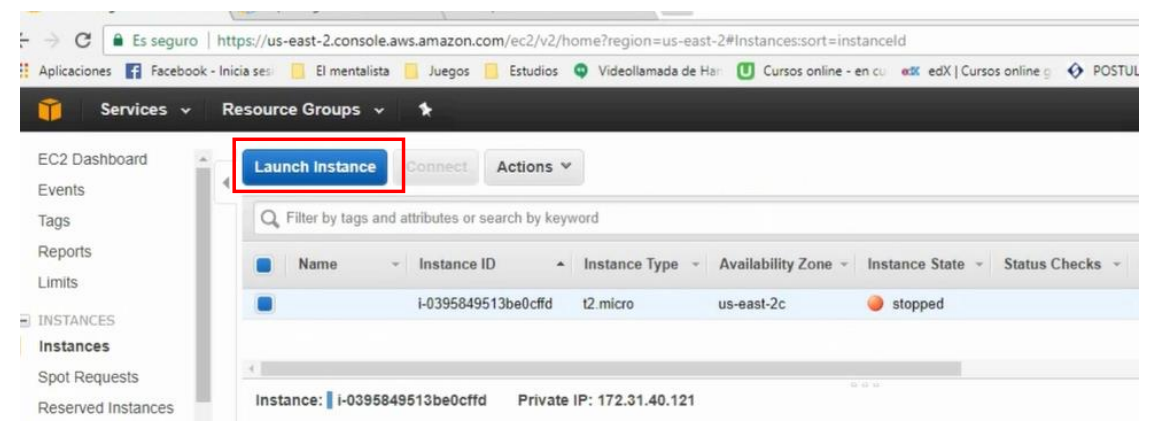

Seleccionar opción My AMIs y busca la plantilla importada (reconocile por el ID de la tarea de importación). 


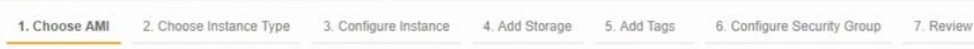

Step 1: Choose an Amazon Machine Image (AMI) Cancel and Exit

An AMl is a template that contains the soffware configuration (operating system, application server, and applications) required to launch your instance. You can select an AMI provided by AWS, our user

community, or the AWS Marketplace; or you can select one of your own AMIs.

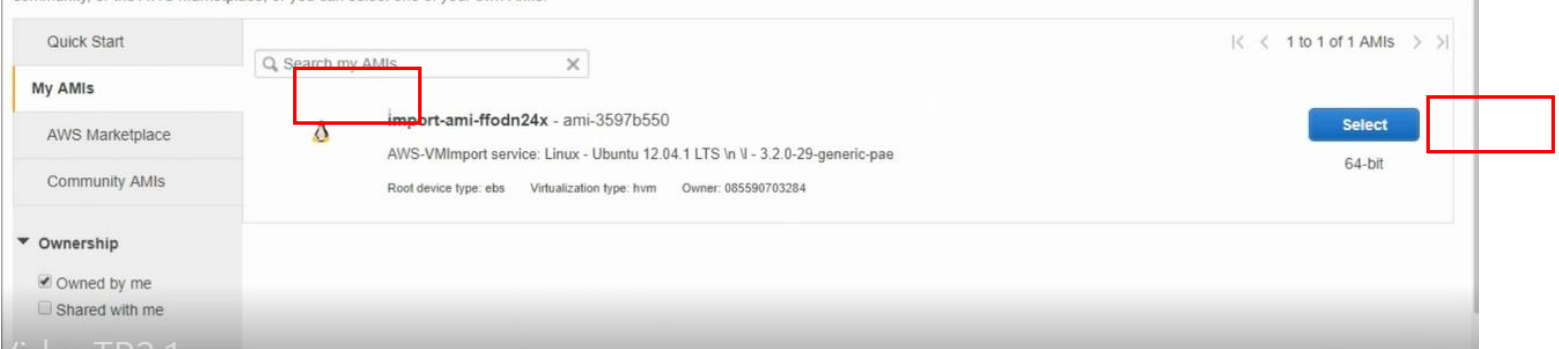

Selecciona las caracteristicas de la instancia a levantar, se debe indicar capacidad de storage, procesamiento, configuración de firewall, entre otros.

Step 6: Configure Security Group

security group is a set of firewall rules that control the traffic for your instance. On this page, you can add rules to allow specific traffic to reach your instance. For example, if you want to set up a web server and Illow Internet traffic to reach your instance, add rules that allow unrestricted access to the HTTP and HTTPS ports. You can create a new security group or select from an existing one below. Learn more about amazon EC2 security groups.

Assign a security group: • Create a new security group

OSelect an existing security group

Security group name: $\quad$ launch-wizard-2

Description: $\quad$ launch-wizard-2 created 2017-09-22T17:34.46.358-05:00

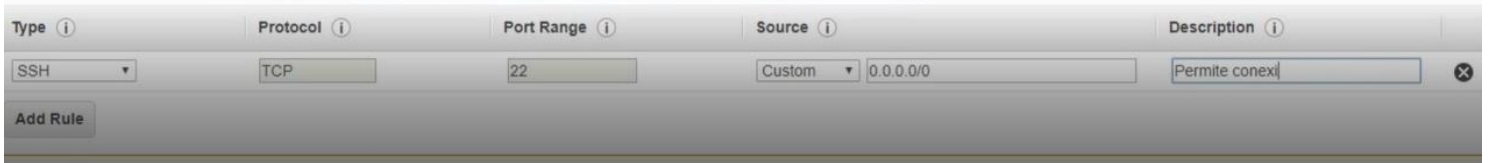

Generar Key Pairs para acceso remoto y lanzar la vm.

\section{Select an existing key pair or create a new key pair}

A key pair consists of a public key that AWS stores, and a private key file that you store. Together, the allow you to connect to your instance securely. For Windows AMIs, the private key file is required to obtain the password used to log into your instance. For Linux AMIs, the private key file allows you to securely $\mathrm{SSH}$ into your instance.

Note: The selected key pair will be added to the set of keys authorized for this instance. Learn more about removing existing key pairs from a public AMI.

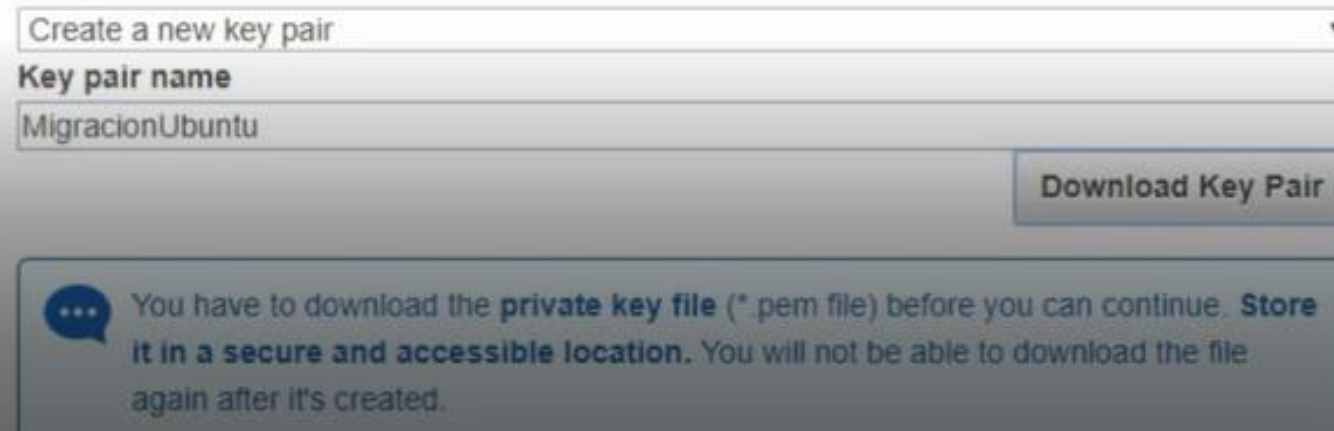

\section{Conexión remota (SSH) usando PuTTy (en caso de Windows RDP)}


Abrir PuTTy e ingresar la IP del servidor al que se conectará

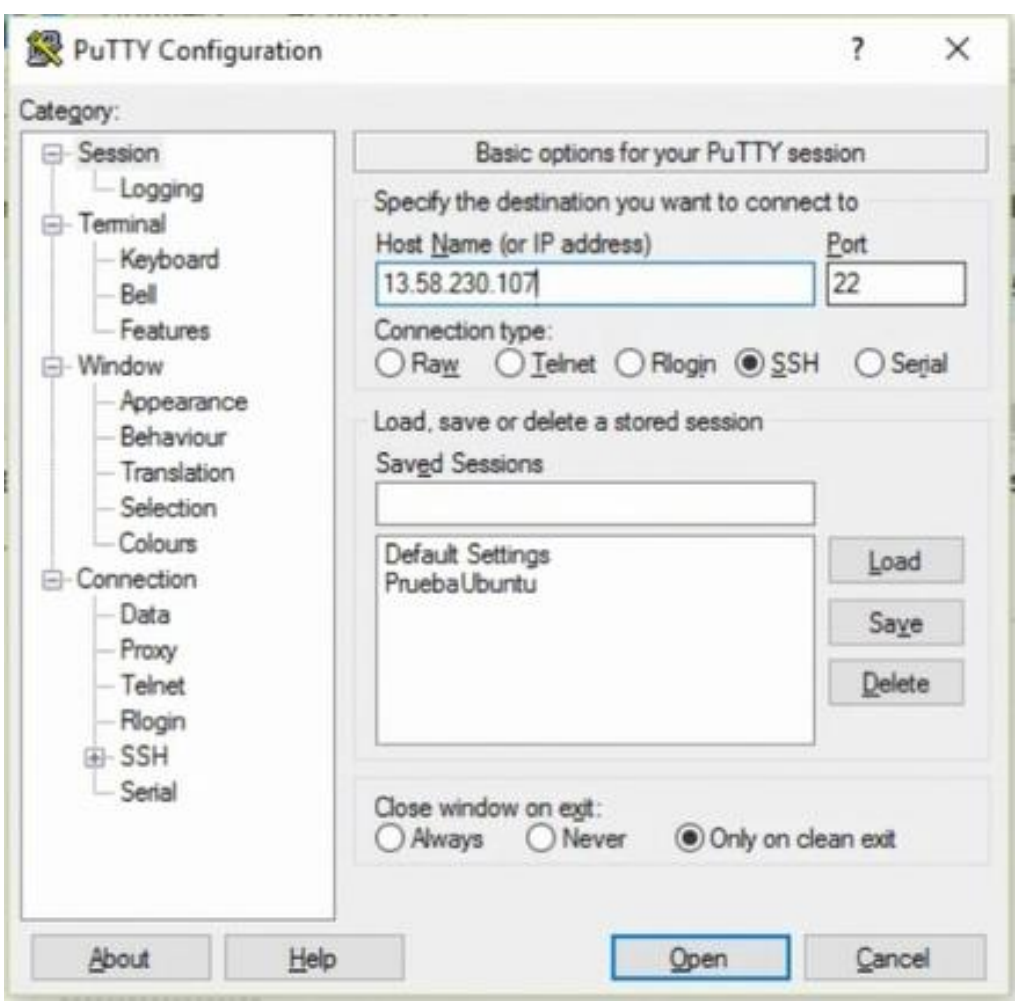

Se le solicitara usuario y password

christian@christian:

login as: christian

christian@13.58,230.107's password:

Welcome to Ubuntu 12.04 .1 LTS (GVO/Linux 3.2.0-29-generic-pae 1686)

* Documentation: https://help.ubuntu.com/

New release ' 14.04 .5 ITS' available.

Run 'do-release-upgrade' to upgrade to it.

Last login: Fri Sep 22 17:38:56 2017 from 190.113.209.223

christianlehristian: $\sim$ mysq 


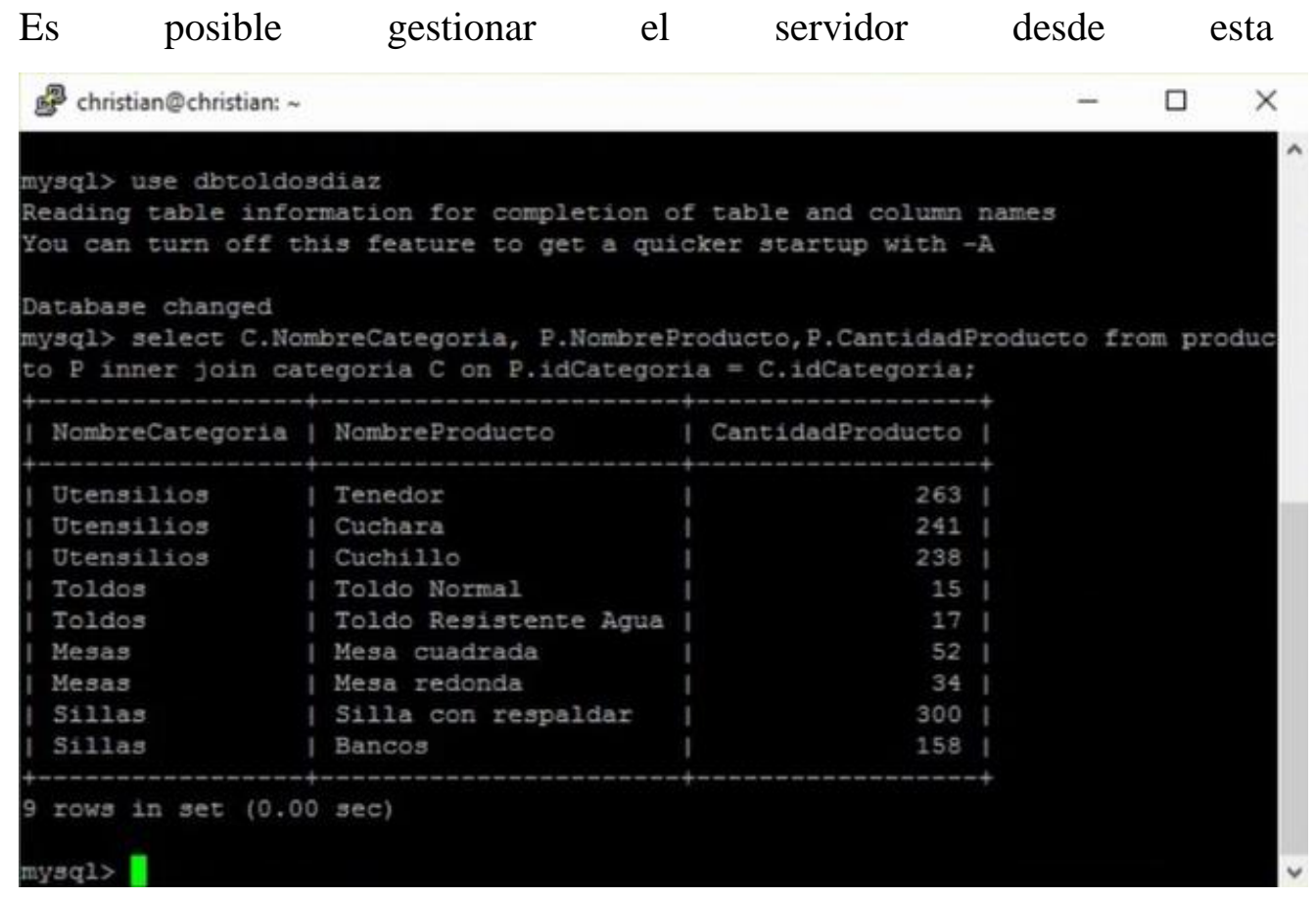

\section{Implementación del Modelo}

Para la implementación del modelo, se uso como caso de uso la empresa Globokas Perú.

A continuación se muestran los entregales completados:

\section{Principios, objetivos y motivadores de negocio}

Ver el anexo 7.

\section{Levantamiento de Información}

Ver el anexo 8.

\section{Especificación de Requerimientos}

Ver el anexo 9.

\section{Benchmarking}

Ver el anexo 10.

\section{Estudio de Viabilidad}

Ver el anexo 11. 
Documento de implementación

Ver el anexo 12. 


\section{CAPITULO 6: RESULTADOS}

En este capítulo, se realiza el análisis de la validación del modelo de migración a la nube de los servidores de una data center, con la literatura y la implementación de esta en un caso de uso. 


\section{Validación del modelo}

\section{Comparación con la literatura}

Al realizarse la investigación, se encontraron otras metodologías para la migración hacia la nube de la infraestructura de una empresa. Estos a su vez se abarcaban en requerimientos, proveedores y hasta en fases. Pero cada uno por su lado, y no los juntaban. Con esa característica, se quiere validar el modelo, puesto que no están abarcando todo en uno solo, y no consideran la evaluación de las cargas. Los modelos encontrados fueron los siguientes:

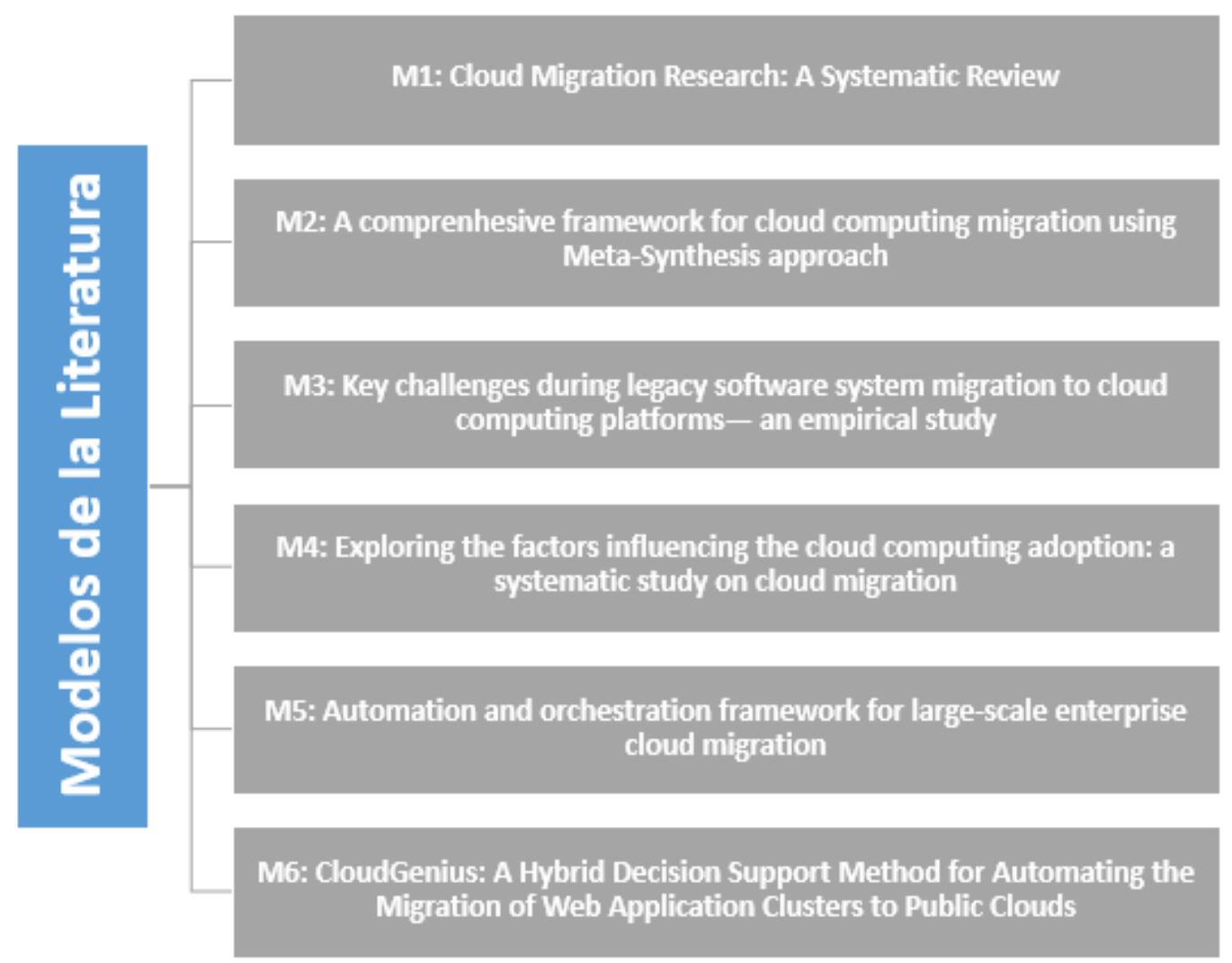

Ilustración 14: Modelos recopilados de la literatura

Fuente: Elaboración Propia 
Cloud Migration Reseach: Como primer modelo, se encontró el de Pooyan Jamshidi, en el cual el resultado de su investigación lo ha colocado en 3 fases: Planeamiento, Ejecución y Evaluación. En la fase de Planeamiento están considerando los requerimientos, los proveedores, los servicios cloud que puedan tener los proveedores y las estrategias que se pueden desarrollar. En la fase de Ejecución, se centran en la extracción de la data; para ello han analizado algoritmos. En la parte de Evaluación, solo están considerando que la migración haya sido exitosa y el testeo correspondiente. Esta recopilación como ya se mencionó en los anteriores, no considera la compatibilidad de las cargas con la plataforma ni la prioridad de estas. Por lo que al no hacer esto está aceptando un riesgo de que pueda ser que haya un retraso en la migración y eso conlleva a un costo que se pueda tener.

A comprenhesive framework for cloud computing migration using Meta-Synthesis approach: Se ha encontrado la investigación de Hamid reza Bazi, en el cual nos muestra un método que contempla: Iniciación, Adopción, Decisión, Migración, Adopción y Soporte. Aquí se detalla la identificación de oportunidades que se pueden dar, desarrollan su estrategia de migración, migran y le dan un control. Pero dentro de estas fases, no están contemplando las cargas de trabajo, si estas son compatibles con la plataforma que se están usando. Aparte en esta investigación se enfocan más en cómo se encuentran en estado de madurez, así que no están abarcando una evaluación de proveedores. También no se centran en un caso de uso, por ende, no contemplan las necesidades ni requerimientos que pueda tener la organización en la cual usarían este método.

Key challenges during legacy software system migration to cloud computing platformsan empirical study: Siguiendo con los métodos, en la investigación de Mahdi Fahmideh Gholami, tienen la definición del AS IS de una organización; quiere decir que contemplan el estado actual de la infraestructura que puedan tener. Abarcan los requerimientos que puedan salir y también el análisis de los proveedores cloud. Ya que es una investigación que abarca más a la evaluación de proveedores y opciones de nube; sigue sin haber una que se encargue de la evaluación y compatibilidad de las cargas con las plataformas. 
Adicional, no lo están colocando en fases por lo que no se mantiene un orden en la migración.

Exploring the factors influencing the cloud computing adoption: a systematic study on cloud migration: Como cuarto modelo se encontró el de Rai Rashmi, el cual propone un modelo conceptual para la migración a la nube, el cual consiste en fases (Análisis de Viabilidad, Análisis de Requerimientos y Plan de Migración, la ejecución de la migración, la validación y el testeo y el monitoreo y mantenimiento), procesos y actividades.

Automation and orchestration framework for large-scale enterprise cloud migration: Los autores proponen Cloud Migration Orchestrator (CMO), el cual es un framework basado en principios de Business Process Management (BPM), cuyo objetivo es automatizar y orquestar las actividades de migración, entre las que se incluyen descubrimiento, análisis, configuración de la pre-migración, ejecución de la migración, configuración de la postmigración y aseguramiento de la calidad.

CloudGenius: A Hybrid Decision Support Method for Automating the Migration of Web Application Clusters to Public Clouds: CloudGenius, que es un método de soporte de decisiones híbrido para automatizar la migración de aplicaciones web a nubes públicas, una característica de este framework es el modelo del proceso de migración evolutivo a cloud, el cual integra los enfoques de migración existentes y métodos para soportar decisiones basados en múltiples criterios, para seleccionar imágenes de VM y servicios de cómputo para diferentes componentes.

Para evaluar y comparar los modelos ya existentes con el propuesto, hemos realizado una comparativa entre ellos según lo siguientes factores obtenidos de la literatura: 


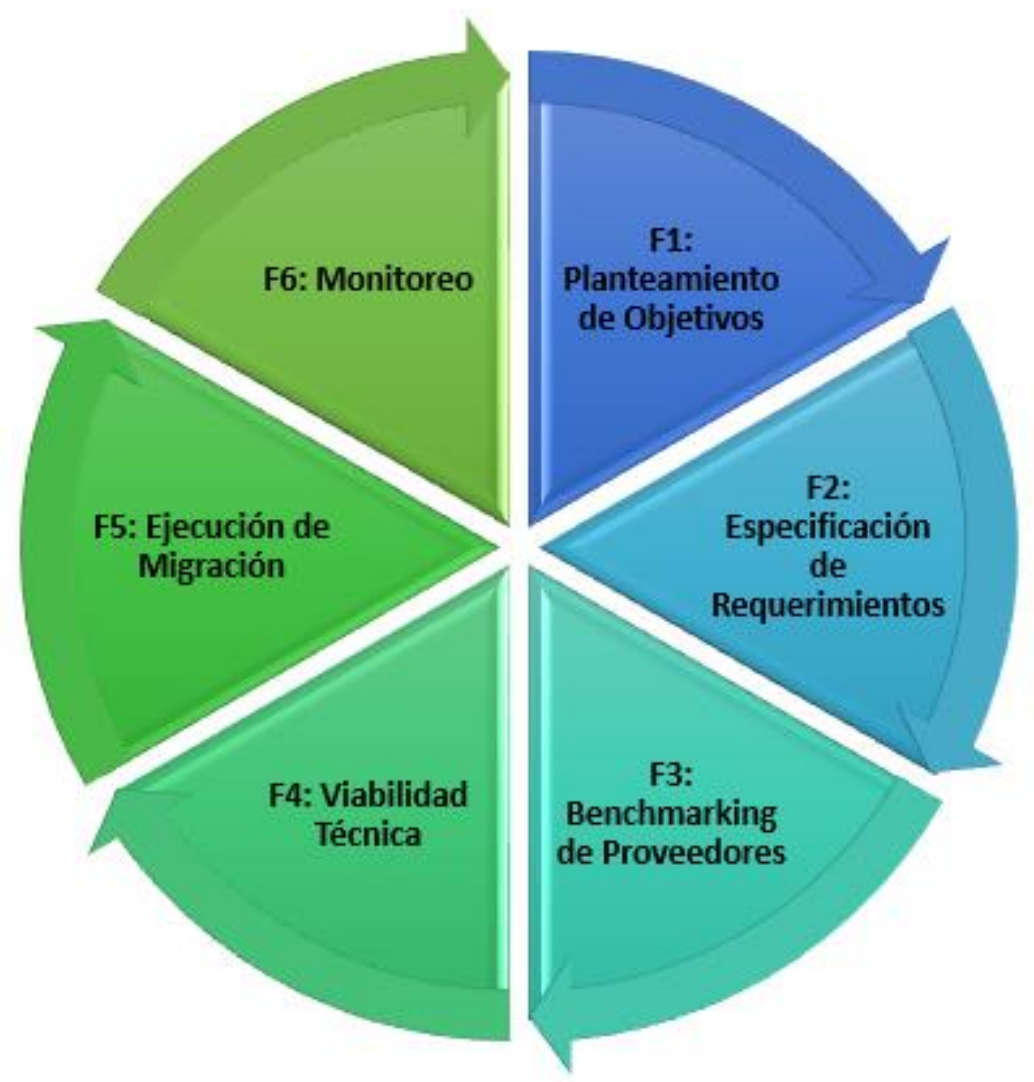

Ilustración 15: Factores de comparación

Fuente: Elaboración Propia

A continuación, se detalla cada factor:

- Planteamiento de Objetivos: Se plantean los objetivos de los stakeholders para el proyecto y el impacto que estos deben tener para el proyecto.

- Especificación de Requerimientos: Se recoge los requerimientos específicos, con el detalle de cada activo de TI para la migración. Aparte se formaliza para su gestión.

- Benchmarking de Proveedores: Se realiza una evaluación para escoger al proveedor y plataforma cloud idónea para la migración. Con las características deseadas por el cliente o usuario.

- Viabilidad Técnica: Se refiere a la compatibilidad entre los componentes de los activos de TI y la de la misma plataforma. 
- Ejecución de Migración: Se realiza la migración, dejando evidencia de ello (Parte técnica)

- Monitoreo: Se realiza el monitoreo y testeo de la migración.

En esta tabla, se muestra la comparación de cada modelo con los factores. Pudiéndose ver que no todos los modelos cumplen con todos los factores, ya que como se menciona anteriormente, se enfocan más en ciertas partes y dejan de lados algunos.

Tabla 4: Comparación de factores con la literatura

\begin{tabular}{|c|c|c|c|c|c|c|c|}
\hline & F1 & F2 & F3 & F4 & F5 & F6 & $\%$ \\
\hline M1 & & $x$ & $x$ & & $x$ & & $50 \%$ \\
\hline M2 & & $x$ & $x$ & & $x$ & $x$ & $66.67 \%$ \\
\hline M3 & $x$ & & $x$ & $x$ & & & $50 \%$ \\
\hline M4 & & $x$ & & & $x$ & $x$ & $50 \%$ \\
\hline M5 & & & $x$ & & $x$ & $x$ & $50 \%$ \\
\hline M6 & $x$ & $x$ & & & $x$ & & $50 \%$ \\
\hline M7 & $x$ & $x$ & $x$ & $x$ & $x$ & $x$ & $100 \%$ \\
\hline
\end{tabular}

Fuente: Elaboración Propia

Leyenda:

M1 Cloud Migration Research: A Systematic Review

F1 Planeamiento de Objetivos

M2

A comprenhesive framework for cloud computing migration using Meta-Synthesis approach

F2 Especificación de requerimientos 
Key challenges during legacy software system migration to cloud computing platforms - an empirical study

Exploring the factors influencing the cloud computing adoption: a systematic study on cloud migration

Automation and orchestration framework for large-scale M5 enterprise cloud migration

CloudGenius: A Hybrid Decision Support Method for

Public Clouds

MMNSDC

\section{Juicio de Expertos}

Adicionalmente a la validación realizada con la comparación de la literatura, se realizó una validación por juicio de expertos, para lo cual se contactó al experto en Cloud, Juan Manuel Cuya Cabanillas, quien ocupa el puesto de IBM Hybrid Cloud Consultant Lead for Spanish South America, en la prestigiosa organización IBM, para la validación utilizó NPS (Net Promoter Score), dicho método establece que la calificación para cada uno de sus criterios debe ser mayor o igual a 9 para considerarla como promotor, entre 7 y 8 para considerarla neutro y menor a 7 para considerarla detractor. Según la evaluación realizada, la puntuación total del modelo fue de 94\%, considerándose aprobada. Para ver el juicio realizado por el experto ver el anexo 13.

\section{Caso de Uso}

A continuación, se presenta el caso de uso para el presente proyecto.

\section{Presentación de Empresa}

La empresa usada para el presente caso de uso es Globokas Perú, una subsidiaria peruana de GTV GlobokasNet LLC cuya sede principal se encuentra en los Estados Unidos de América, actualmente cuenta con 10 años de operación en el país peruano. Globokas cuenta con cobertura nacional y cuenta con la capacidad de desplegar agentes "Kasnet" 
en cualquier zona, ya sea esta urbana o rural en el Perú; actualmente llegan a amas de 6000 localidades del Perú.

Globokas es una PYME perteneciente al sector financiero, cuya principal actividad es gestionar la red más grande de agentes corresponsales del país, operan con diferentes entidades del sector financiero peruano como BBVA, Scotiabank, Banbif, Mi Banco, Banco Financiero, entre otros.

Entre los servicios que brindan a sus clientes se encuentran los servicios de:

- Optimización de canales de distribución

- Descongestión de red de agencias

- Bancarizar

\section{Implementación}

\section{Fase de Entendimiento}

En primer lugar, se procedió a ejecutar la fase de Entendimiento del método propuesto, el cual está compuesto por los siguientes documentos: Principios, objetivos y motivadores de negocio y Levantamiento de Información. Para el documento de Principios, objetivos y motivadores de negocio se completaron los respectivos datos, como por ejemplo los siguientes Business Drivers: reducir costos de TI, optimizar el centro de datos, optimizar disponibilidad de los servicios críticos, entre otros.

En el documento Levantamiento de Información se levantan los activos de TI de la organización, se realiza el diagrama de la arquitectura física, y la configuración de firewall que posee. En el caso de estudio utilizado se tienen 18 servidores físicos, en el cual se detallan sus características como sistema operativo, RAM, disco duro, servicios, etc.

\section{Fase de Exploración}

Una vez que se realizó ambos documentos, se pasó a la fase de Exploración, en la cual se identifican y formalizan los requerimientos, para esto se hace uso de la plantilla: Especificación de Requerimientos, en la cual se explican los antecedentes, el objetivo del requerimiento, el alcance funcional, requerimientos funcionales, no funcionales, alcance 
técnico y exclusiones, en el caso de la empresa que se usó para la validación, el objetivo del requerimiento busca migrar a una nube pública sus servidores.

\section{Fase de Evaluación}

A continuación, se pasó a la fase de Evaluación, donde se procedió a realizar el benchmarking de las plataformas cloud a fin de determinar la mejor opción para la organización, para esto se cuenta con una plantilla de benchmarking, la cual se basa en la ISO 25000 SQuaRE (System and Software Quality Requirements and Evaluation) y adicionalmente en el precio, de esta evaluación la plataforma ganadora fue AWS (Amazon Web Services).

Una vez decidida la plataforma se debe completar el estudio de viabilidad, en el cual se evalúan la viabilidad técnica, financiera y operativo, para este punto se puede utilizar las herramientas que brinda el método propuesto, en la viabilidad técnica se evalúan los siguientes temas: si es un servidor virtualizado, el sistema operativo, el formato de exportación del servidor, el disco duro del servidor, estos datos nos permiten calcular que tan factible es su migración y permite seleccionar las cargas de trabajo a migrar. En la viabilidad financiera se calculan los costes actuales del proyecto, y los beneficios que este brinda, así se crea un ROI (Return On Investment) que permite determinar en cuanto tiempo se recupera la inversión. Por otro lado, la viabilidad operativa consiste en determinar si las operaciones una vez se implemente el proyecto se podrán realizar correctamente.

\section{Fase de Migración}

Cuando ya se haya determinado la plataforma y cargas de trabajo a migrar, se pasó a la fase de Migración, en la cual se procedió a migrar a la plataforma AWS, para esto se necesitó utilizar EC2-API-TOOLS que es un API de AWS EC2, una vez instalado esto, por medio de scripts desde el CMD es posible crear roles y políticas en la cuenta de AWS para poder importar el archivo del servidor, luego de tener el rol con las políticas correctas, se procedió a importar el archivo del disco duro del servidor usando un script copy, y con un script esta se importó en una imagen que luego se puede desplegar asignando los recursos que se deseen. Todo el proceso de la migración se documentó haciendo uso de la plantilla: Documento de implementación, el cual cuenta con una sección de conclusiones, en la cual se especificó que la migración fue satisfactoria. 


\section{Resultados}

\section{Riesgos}

A continuación, se detallan los resultados obtenidos del proyecto, para esto se utilizó como base la información brindada en la literatura, y esta fue comparada con los datos sacados del caso de uso, como resultado se puede apreciar la reducción de probabilidad de los principales riesgos durante la migración a la nube.

Tabla 5: Riesgos

Fuente: Elaboración Propia

Con

el

\begin{tabular}{|l|l|l|}
\hline \multirow{2}{*}{ RIESGOS } & \multicolumn{2}{l|}{ PROBABILIDAD } \\
\cline { 2 - 4 } & LITERATURA & MMNSDC \\
\hline Mala recopilación de los requerimientos & $50 \%$ & $15 \%$ \\
\hline Mala asignación de recursos & $40 \%$ & $5 \%$ \\
\hline Stand By por incompatibilidad de plataforma con las & & $10 \%$ \\
\hline cargas de trabajo & $30 \%$ & $5 \%$ \\
\hline Falta de seguridad en la plataforma cloud & $20 \%$ & $10 \%$ \\
\hline Poco entendimiento del método & & $5 \%$ \\
\hline No cumpla con los objetivos de la organización & $60 \%$ & $15 \%$ \\
\hline
\end{tabular}

análisis realizado, se puede concluir que con el modelo se ha reducido hasta en un $45 \%$ los riesgos existentes durante la migración.

\section{Beneficio}

Cuando se empezó la implementación del método, se utilizó las plantillas que estas contemplan. Y en dicha validación en el caso de uso, se determinó que los beneficios para el caso de uso fueron los siguientes: 
Costo Beneficio:

Al realizar el presupuesto del proyecto en el caso para el caso de uso. El costo total de este proyecto fue de S/. 57,871.97. En el cual estamos incluyendo tanto la adquisición de infraestructura, y los costos de los roles. Al tener los roles definidos se reducen costos ya que no repiten actividades ni doble esfuerzo por el cual el proyecto se atrasaría. También se planifica el proyecto para que tenga una duración de 2 meses.

\section{Presupuesto del Proyecto}

\section{COSTOS DE SOFTWARE HARDWARE Y PERSONAL}

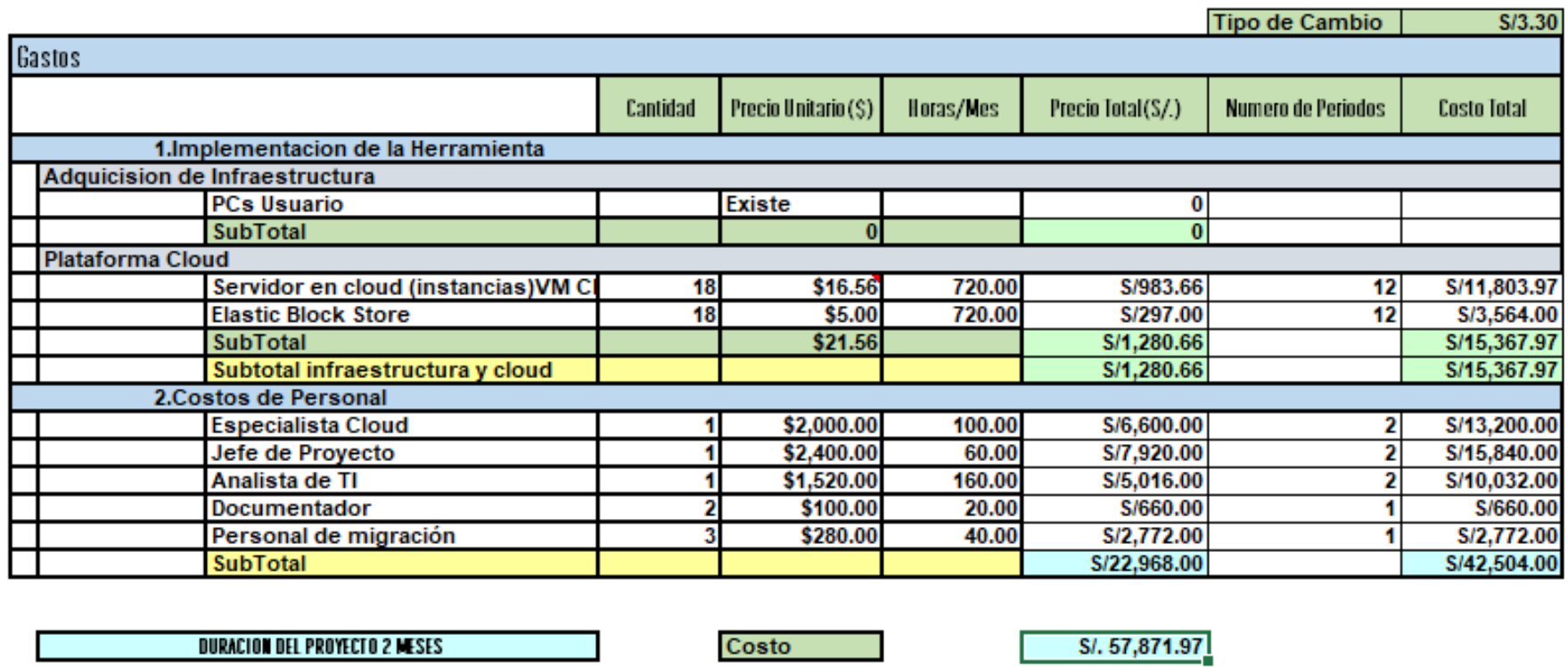

Fuente: Elaboración Propia

El beneficio que se tiene es de S/. 137,316 anual, el cual incluye los ahorros de electricidad, tanto para el sistema de refrigeración como para los servidores, este monto anual corresponde a S/. 26,916. Por otro lado, el mantenimiento de los servidores anualmente es de S/. 108,000, los cuales incluye el personal que revisa los servidores y el mantenimiento de estos; adicionalmente, la reposición de infraestructura que se tiene que realizar para el hardware dañado o en caso crezca la red corresponde a S/. 2400. 


\section{Beneficios}

CUADRODE BENEFICIOS PARA UN CLIENTE QUE MIGRA SUS SERVIDORES A CLOUD

\begin{tabular}{|c|c|c|c|c|}
\hline GASTOS DE MANIENIMIENTO & MEIRICA & COSTO UNITARIO & COSTO MENSUAL & COSTO ANUAL \\
\hline Electricidad de sistema de refrigeración & $15 \mathrm{Kwh}$ & $2,000.00$ & $2,000.00$ & S/. $\quad 24,000.00$ \\
\hline Electricidad de los servidores & 18 servidores & 13.50 & 243.00 & S/. $\quad 2,916.00$ \\
\hline Mantenimiento de servidores & 18 servidores & 500.00 & $9,000.00$ & S/. $108,000.00$ \\
\hline Reposicion de infraestructura & 1Rep & 200.00 & 200.00 & S/. $\quad 2,400.00$ \\
\hline & & Total & S/. $\quad 11,443.00$ & S/. $137,316.00$ \\
\hline
\end{tabular}

\begin{tabular}{|l|r|r|r|}
\cline { 3 - 4 } & & \multicolumn{1}{c}{ ROI Mensual } & \multicolumn{1}{c}{ ROI Anual } \\
\hline Inversión & $\mathrm{S} / 57,871.97$ & $19.77 \%$ & $237.28 \%$ \\
\hline
\end{tabular}

Ilustración 16: Beneficios del Proyecto

Fuente: Elaboración Propia

En el análisis elaborado se tiene que el ROI mensual es de $19.7 \%$, lo que quiere decir que es positivo y está generando rentabilidad a la empresa. Y la recuperación de esta inversión se origina a partir del mes 6 luego de la finalización del proyecto o de mes 8 desde el inicio del proyecto.

\section{Retorno de Inversión}

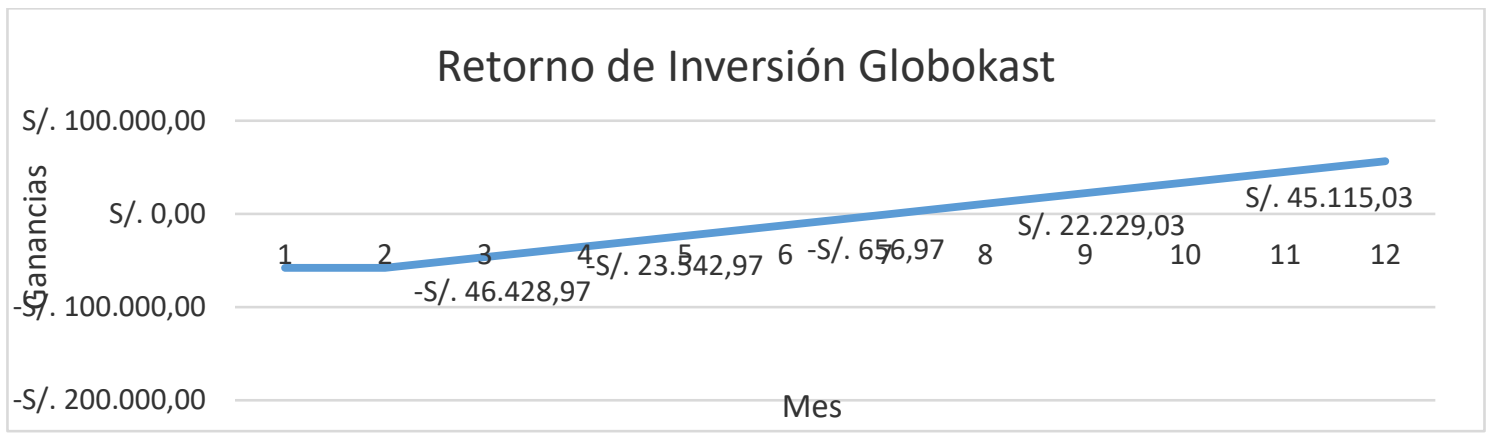

Ilustración 17: Retorno de Inversión

Fuente: Elaboración Propia 


\section{Alineamiento Estratégico}

El modelo al tener el documento: Principios, objetivos y motivadores de negocio busca realizar un alineamiento estratégico, para ello se le pide al cliente sus objetivos para este proyecto. Los cuales vienen de las necesidades que pueda tener la empresa con respecto al proyecto. Estos han sido comparados con los objetivos que tiene el modelo de migración propuesto: Reducción de costos de TI, Disminución de Riesgos, Alineamiento Estratégico y Mejora continua.

\section{Alineamiento de Objetivos del Modelo}

\begin{tabular}{|c|c|c|c|c|c|}
\hline \multicolumn{2}{|r|}{ OBJETIVOS DEL MODELO } & \multirow{3}{*}{$\begin{array}{c}\begin{array}{c}\text { Reducción de } \\
\text { costos de TI }\end{array} \\
\mathrm{X} \\
\end{array}$} & \multirow{3}{*}{$\begin{array}{c}\text { Disminución de } \\
\text { Riesgos }\end{array}$} & \multirow{3}{*}{$\begin{array}{l}\text { Alineamiento } \\
\text { Estrategico }\end{array}$} & \multirow{2}{*}{$\begin{array}{c}\text { Tener una } \\
\text { mejora continua }\end{array}$} \\
\hline \multicolumn{2}{|r|}{ OBJETIVOS DEL PROYECTO } & & & & \\
\hline OGK1 & Reducir costos de TI & & & & \\
\hline OGK2 & Optimizar el centro de datos & $\mathrm{x}$ & $\mathrm{x}$ & & $\mathrm{x}$ \\
\hline OGK3 & Disponibilidad optimizada de los servicios criticos & & $\mathrm{x}$ & & $\mathrm{x}$ \\
\hline OGK4 & $\begin{array}{l}\text { Creación de una organización más agil basada en la } \\
\text { automatización de su DC }\end{array}$ & & & $\mathrm{x}$ & $\mathrm{x}$ \\
\hline OGK5 & Ventaja Competitiva & $\mathrm{x}$ & $\mathrm{x}$ & $\mathrm{x}$ & $\mathrm{x}$ \\
\hline OGK6 & \begin{tabular}{|l|} 
Integración de sus servicios de TI (Almacenamiento \\
servidores, redes, energia, interoperabilidad)
\end{tabular} & & $\mathrm{x}$ & $x$ & $\mathrm{x}$ \\
\hline
\end{tabular}

Ilustración 18: Alineamiento de Objetivos del Modelo

Fuente: Elaboración Propia

Al realizar el análisis en la comparativa de los objetivos del modelo como los de la empresa para el proyecto. Se observa que todos los objetivos del modelo se relacionan con por lo menos uno de los objetivos del negocio para el proyecto, por lo tanto, cumple con un $100 \%$ en general. Para poner a prueba el alineamiento, se habló con el Key User de la empresa: Jimmy Novoa, para que lo completara, según su experiencia con la implementación.

También se comparó los objetivos del proyecto con los objetivos generales de la organización. Del cual se tuvo que cumple con el 100\%. Se observa que cada uno de los objetivos del proyecto se relaciona con al menos un objetivo general de la empresa. Al igual que en el anterior, se pidió al Key User que nos dijera los objetivos que tienen como organización y se le pidió que completara el siguiente cuadro: 


Alineamiento loS Objetivos
\begin{tabular}{|c|l|c|c|c|c|c|}
\hline \multicolumn{1}{|c|}{ OBJETIVOS DEL PROYECTO } & $\begin{array}{c}\text { Superar a la } \\
\text { competencia en } \\
\text { visibilidad } \mathrm{y} \\
\text { ventas dentro } \\
\text { del mercado }\end{array}$ & $\begin{array}{c}\text { Incursionar en } \\
\text { las ventas on- } \\
\text { line con un } \\
\text { margen de éxito } \\
\text { sostenible }\end{array}$ & $\begin{array}{c}\text { Reducir al } \\
\text { mínimo los } \\
\text { gastos y recortar } \\
\text { el déficit en al } \\
\text { menos un 40\% }\end{array}$ & $\begin{array}{c}\text { Ser una marca } \\
\text { que los } \\
\text { demandantes } \\
\text { reconozcan por } \\
\text { su innovación }\end{array}$ & $\begin{array}{c}\text { Mejorar la } \\
\text { rentabilidad del } \\
\text { área de TI, en un } \\
3 \% \text { en el año } \\
2018\end{array}$ \\
\hline OGK1 & Reducir costos de TI & & $\mathrm{X}$ & $\mathrm{X}$ & & $\mathrm{X}$ \\
\hline OGK2 & Optimizar el centro de datos & & $\mathrm{X}$ & $\mathrm{X}$ & $\mathrm{X}$ & $\mathrm{X}$ \\
\hline OGK3 & Disponibilidad optimizada de los servicios criticos & & & $\mathrm{X}$ & $\mathrm{X}$ & $\mathrm{X}$ \\
\hline OGK4 & $\begin{array}{l}\text { Creación de una organización más agil basada en la } \\
\text { automatización de su DC }\end{array}$ & $\mathrm{X}$ & $\mathrm{X}$ & & $\mathrm{X}$ & \\
\hline OGK5 & Ventaja Competitiva & $\mathrm{X}$ & $\mathrm{X}$ & $\mathrm{X}$ & $\mathrm{X}$ & \\
\hline OGK6 & $\begin{array}{l}\text { Integración de sus servicios de TI (Almacenamiento } \\
\text { servidores, redes, energia, interoperabilidad) }\end{array}$ & $\mathrm{X}$ & & $\mathrm{X}$ & $\mathrm{X}$ & $\mathrm{X}$ \\
\hline
\end{tabular}

Ilustración 19: Alineamiento con los Objetivos Generales de la Empresa

Fuente: Elaboración Propia 


\section{CAPÍTULO 7: GESTIÓN DEL PROYECTO}

En este capítulo se explica a detalle la gestión del desarrollo del proyecto bajo los lineamientos de la metodología PMBOK. Se describen los planes de gestión, matrices, entre otros documentos necesarios para el desarrollo de este proyecto. 


\section{REGISTRO DE INTERESADOS}

En este documento se presenta la información de todos los interesados, así como su influencia e impacto en el desarrollo del proyecto. A continuación, se presenta el registro de interesados.

Tabla 6: Registro de Interesados

\begin{tabular}{|c|c|c|c|c|c|c|c|c|c|c|c|c|}
\hline \multirow{2}{*}{ Nombres y Apellidos } & \multirow{2}{*}{ Organización } & \multirow{2}{*}{ Cargo } & \multirow{2}{*}{ Información del contacto } & \multirow{2}{*}{ Requerimientos sobre el Producto } & \multicolumn{5}{|c|}{ Influencia } & \multirow{2}{*}{\begin{tabular}{|l|} 
Influencia \\
sobre el \\
proyecto
\end{tabular}} & \multirow{2}{*}{\begin{tabular}{|l} 
Impacto \\
el \\
proyecto
\end{tabular}} & \multirow{2}{*}{$\begin{array}{l}\text { Tipo de } \\
\text { interés }\end{array}$} \\
\hline & & & & & I & $\mathbf{P}$ & $\mathbf{E}$ & $\mathbf{S}$ & $\mathbf{C}$ & & & \\
\hline Fabiola Loo Cuya & Data Center & Jefe de Proyecto & u201310485@upc.edu.pe & $\begin{array}{l}\text { Analizar, ejecutar y supervisar el desarrollo del proyecto con el } \\
\text { fin de cumplir los objetivos en las fechas indicadas. }\end{array}$ & 凶 & 凶 & 凶 & 凶 & 凶 & Favorable & Alto & $\begin{array}{l}\text { Conseguir } \\
\text { toda la } \\
\text { información } \\
\text { requerida para } \\
\text { el desarrollo } \\
\text { exitoso del } \\
\text { proyecto. }\end{array}$ \\
\hline Christian Rojas Solórzano & Data Center & Jefe de proyecto & u201311715@upc.edu.pe & $\begin{array}{l}\text { Analizar, ejecutar y supervisar el desarrollo del proyecto con el } \\
\text { fin de cumplir los objetivos en las fechas indicadas. }\end{array}$ & 凶 & 凶 & 凶 & 凶 & 凶 & Favorable & Alto & $\begin{array}{l}\text { Conseguir } \\
\text { toda la } \\
\text { información } \\
\text { requerida para } \\
\text { el desarrollo } \\
\text { exitoso del } \\
\text { proyecto. }\end{array}$ \\
\hline
\end{tabular}




\begin{tabular}{|c|c|c|c|c|c|c|c|c|c|c|c|c|}
\hline $\begin{array}{l}\text { Rosario Villalta } \\
\text { Jimmy Armas } \\
\text { Pedro ShIguihara }\end{array}$ & UPC & Comité & $\begin{array}{l}\text { rosario.villalta@upc.edu.pe } \\
\text { jimmy.armas@upc.edu.pe } \\
\text { pedro.shiguihara@upc.edu.pe }\end{array}$ & $\begin{array}{l}\text { Evaluar el proyecto bajo los estándares establecidos de la EISC } \\
\text { de la UPC }\end{array}$ & 凶 & $\square$ & $\square$ & 凶 & $\square$ & Favorable & Alto & $\begin{array}{l}\text { Formar } \\
\text { profesionales } \\
\text { que cumplan } \\
\text { los Students } \\
\text { Outcomes por } \\
\text { el cual se rige } \\
\text { la EISC }\end{array}$ \\
\hline Paul Rivas & Data Center & Profesor Gerente & pcsipriv@upc.edu.pe & Brindar apoyo y asesoría durante el desarrollo del proyecto & 凶 & $\square$ & 凶 & 凶 & 凶 & Favorable & Regular & $\begin{array}{l}\text { Culminación } \\
\text { de todos los } \\
\text { proyectos } \\
\text { pertenecientes } \\
\text { a la empresa } \\
\text { virtual DATA } \\
\text { CENTER }\end{array}$ \\
\hline Edgar Díaz & UPC & Profesor Cliente & pcsiedia@upc.edu.pe & Brindar apoyo y asesoría durante el desarrollo del proyecto & 凶 & $\square$ & $\square$ & 凶 & 凶 & Favorable & Alto & $\begin{array}{lr}\text { Solución } & \text { de } \\
\text { dudas } & \text { y } \\
\text { asesoramiento } & \\
\text { durante } & \text { el } \\
\text { desarrollo } & \text { del } \\
\text { proyecto }\end{array}$ \\
\hline Emilio Herrera & $\begin{array}{l}\text { Empresas } \\
\text { Virtuales }\end{array}$ & Gerente general & pcsieher@upc.edu.pe & $\begin{array}{l}\text { Evaluar el proyecto, bajo los estándares establecidos de la EISC } \\
\text { de la UPC }\end{array}$ & 凶 & $\square$ & $\square$ & 凶 & 凶 & Favorable & Regular & $\begin{array}{l}\text { Gestión de los } \\
\text { proyectos de } \\
\text { las empresas } \\
\text { virtuales }\end{array}$ \\
\hline
\end{tabular}


Fuente: Elaboración Propia

\section{MATRIZ DE TRAZABILIDAD DE REQUERIMIENTOS}

A continuación, se muestra un análisis de la matriz de trazabilidad de requerimientos, esta matriz vincula los requerimientos del proyecto con los objetivos y entregables a desarrollar.

Tabla 7: Matriz de trazabilidad de requerimientos

\begin{tabular}{|c|c|c|c|c|c|c|c|c|c|}
\hline ID & Requerimiento & Justificación & $\begin{array}{l}\text { Prioridad/ } \\
\text { Categoría }\end{array}$ & $\begin{array}{l}\text { Propósitos u Objetivos } \\
\text { del proyecto }\end{array}$ & Código EDT & $\begin{array}{l}\text { Método de } \\
\text { Revisión }\end{array}$ & $\begin{array}{l}\text { Verificación de Alcance } \\
\text { / Validación }\end{array}$ & Estado Actual & Fecha \\
\hline RQ01 & $\begin{array}{l}\text { Se requiere analizar } \\
\text { la planes de } \\
\text { migración a la nube } \\
\text { existentes y el } \\
\text { funcionamiento de } \\
\text { una cloud hibrida }\end{array}$ & $\begin{array}{l}\text { Es necesario entender como } 1 \\
\text { funciona una cloud hibrida y } \\
\text { los planes de migración } \\
\text { existentes para poder plantear } \\
\text { una solución. }\end{array}$ & Alta & $\begin{array}{l}\text { OE1: Analizar las buenas } \\
\text { prácticas de migración a } \\
\text { la nube }\end{array}$ & 1.3.1 & Presencial & Cumplido & Activo & $13 / 04 / 2017$ \\
\hline RQ02 & $\begin{array}{l}\text { Se requiere diseñar } \\
\text { un modelo de }\end{array}$ & $\begin{array}{l}\text { El modelo permitirá un } \\
\text { conjunto de pasos y buenas }\end{array}$ & Alta & $\begin{array}{l}\text { OE2: Diseñar un modelo } \\
\text { de migración hacia cloud }\end{array}$ & 1.3.2 & Presencial & Cumplido & Activo & 13/04/2017 \\
\hline
\end{tabular}




\begin{tabular}{|c|c|c|c|c|c|c|c|c|c|}
\hline & $\begin{array}{l}\text { migración a la nube } \\
\text { de servidores de un } \\
\text { data center }\end{array}$ & $\begin{array}{l}\text { prácticas para que cualquier } \\
\text { organización pueda migar } \\
\text { sus servidores a la nube }\end{array}$ & & $\begin{array}{l}\text { de los servidores de un } \\
\text { data center. }\end{array}$ & & & & & \\
\hline RQ03 & $\begin{array}{l}\text { Se requiere } \\
\text { implementar el } \\
\text { diseño para luego } \\
\text { poder validarlo }\end{array}$ & $\begin{array}{l}\text { El modelo debe ser validado } \\
\text { para garantizar que funciona } \\
\text { correctamente }\end{array}$ & Alta & $\begin{array}{l}\text { OE3: Validar el modelo } \\
\text { de migración a la nube }\end{array}$ & 1.3.7 & Presencial & Cumplido & Activo & $13 / 04 / 2017$ \\
\hline RQ04 & $\begin{array}{l}\text { Se requiere } \\
\text { proponer un plan de } \\
\text { continuidad para el } \\
\text { proyecto }\end{array}$ & $\begin{array}{l}\text { El plan de continuidad } \\
\text { garantizara el funcionamiento } \\
\text { del modelo a través del } \\
\text { tiempo }\end{array}$ & Alta & $\begin{array}{l}\text { OE4: Proponer un plan de } \\
\text { continuidad que garantice } \\
\text { la funcionalidad del } \\
\text { modelo }\end{array}$ & 1.4 .2 & Presencial & Cumplido & Activo & $13 / 04 / 2017$ \\
\hline
\end{tabular}

Fuente: Elaboración Propia

\section{MATRIZ DE ASIGNACIÓN DE RESPONSABILIDADES}

A continuación, se presenta la matriz de asignación de responsabilidades de todos los involucrados del proyecto, estas responsabilidades son designadas en función al entregable respectivo.

Función que realiza el rol en el entregable: 
$\boldsymbol{R}$ (Es el responsable del entregable), $\boldsymbol{C}$ (Coordina actividades del entregable), $\boldsymbol{P}$ (Participa en la construcción/elaboración del entregable), $\boldsymbol{A}$ (Aprueba entregable), $\boldsymbol{V}$ (Participa en el control de calidad del entregable), $\boldsymbol{F}$ (El entregable requiere su firma).

Tabla 8: Matriz de asignación de responsabilidades

\begin{tabular}{|c|c|c|c|c|c|c|}
\hline Código EDT & Entregable & Jefe de Proyecto & Comité de Proyectos & $\begin{array}{l}\text { Profesor Gerente } \\
\text { DATACENTER }\end{array}$ & $\begin{array}{l}\text { Profesor } \\
\text { Cliente }\end{array}$ & $\begin{array}{lll}\text { Recursos } & \text { de } & \text { IT } \\
\text { Service } & & \end{array}$ \\
\hline 1.1 & Inicio & & & & & \\
\hline 1.1.1 & Project Charter & $\mathrm{R}$ & A & $\mathrm{A}, \mathrm{C}, \mathrm{F}, \mathrm{V}$ & $\mathrm{A}, \mathrm{C}, \mathrm{F}, \mathrm{V}$ & V \\
\hline 1.2 & Planificación & & & & & \\
\hline 1.2 .1 & Cronograma & $\mathrm{R}$ & A & $\mathrm{A}, \mathrm{C}, \mathrm{F}$ & V & V \\
\hline 1.2 .2 & Diccionario EDT & $\mathrm{R}$ & A & $\mathrm{A}, \mathrm{C}, \mathrm{F}$ & $\mathrm{V}$ & $\mathrm{V}$ \\
\hline 1.2 .3 & $\begin{array}{l}\text { Plan de gestión del } \\
\text { alcance }\end{array}$ & $R$ & A & $\mathrm{A}, \mathrm{C}, \mathrm{F}$ & V & V \\
\hline 1.2 .4 & $\begin{array}{l}\text { Plan de gestión de } \\
\text { RRHH }\end{array}$ & $\mathrm{R}$ & A & $\mathrm{A}, \mathrm{C}, \mathrm{F}$ & V & V \\
\hline 1.2 .5 & $\begin{array}{l}\text { Plan de gestión de } \\
\text { comunicaciones }\end{array}$ & $\mathrm{R}$ & A & $\mathrm{A}, \mathrm{C}, \mathrm{F}$ & V & V \\
\hline
\end{tabular}




\begin{tabular}{|c|c|c|c|c|c|c|}
\hline 1.2 .6 & $\begin{array}{ll}\text { Registro } & \text { de } \\
\text { interesados }\end{array}$ & $\mathrm{R}$ & A & A,C,F & V & V \\
\hline 1.2 .7 & $\begin{array}{l}\text { Plan de gestión de } \\
\text { riesgo }\end{array}$ & $\mathrm{R}$ & A & A,C,F & V & V \\
\hline 1.2 .8 & $\begin{array}{l}\text { Descripción de roles y } \\
\text { responsabilidades }\end{array}$ & $\mathrm{R}$ & A & A,C,F & $\mathrm{V}$ & $\mathrm{V}$ \\
\hline 1.2 .9 & $\begin{array}{l}\text { Plan de gestión del } \\
\text { cronograma }\end{array}$ & $\mathrm{R}$ & A & A,C,F & V & V \\
\hline 1.2 .10 & $\begin{array}{l}\text { Plan de gestión de } \\
\text { calidad }\end{array}$ & $\mathrm{R}$ & A & A,C,F & V & V \\
\hline 1.2 .11 & Matriz RAM & $\mathrm{R}$ & A & A,C,F & V & V \\
\hline 1.2 .12 & $\begin{array}{l}\text { Matriz de trazabilidad } \\
\text { de requerimientos }\end{array}$ & $\mathrm{R}$ & A & A,C,F & V & V \\
\hline 1.2 .13 & Matriz de riesgos & $\mathrm{R}$ & A & A,C,F & V & V \\
\hline 1.2 .14 & $\begin{array}{ll}\text { Matriz de } \\
\text { comunicaciones }\end{array}$ & $\mathrm{R}$ & A & A,C,F & V & V \\
\hline 1.3 & Ejecución & & & & & \\
\hline 1.3 .1 & $\begin{array}{l}\text { Documento de buenas } \\
\text { prácticas de migración } \\
\text { a la nube }\end{array}$ & $\mathrm{R}$ & A & $\mathrm{A}, \mathrm{C}, \mathrm{F}$ & V & V \\
\hline
\end{tabular}




\begin{tabular}{|c|c|c|c|c|c|c|}
\hline 1.3 .2 & $\begin{array}{l}\text { Modelo de Migración } \\
\text { a la Nube de los } \\
\text { Servidores de un Data } \\
\text { Center }\end{array}$ & $\mathrm{R}$ & A & $\mathrm{A}, \mathrm{C}, \mathrm{F}$ & V & V \\
\hline 1.3 .3 & $\begin{array}{l}\text { Levantamiento de } \\
\text { información }\end{array}$ & $\mathrm{R}$ & A & $\mathrm{A}, \mathrm{C}, \mathrm{F}$ & V & V \\
\hline 1.3 .4 & $\begin{array}{lr}\text { Documento } & \text { de } \\
\text { requerimientos } & \text { del } \\
\text { cliente } & \end{array}$ & $\mathrm{R}$ & A & $\mathrm{A}, \mathrm{C}, \mathrm{F}$ & $\mathrm{V}$ & V \\
\hline 1.3 .5 & $\begin{array}{l}\text { Benchmarking de } \\
\text { proveedores }\end{array}$ & $\mathrm{R}$ & A & A,C,F & $\mathrm{V}$ & V \\
\hline 1.3 .6 & Estudio de viabilidad & $\mathrm{R}$ & $\mathrm{A}$ & $\mathrm{A}, \mathrm{C}, \mathrm{F}$ & $\mathrm{V}$ & V \\
\hline 1.3 .7 & $\begin{array}{l}\text { Acta de validación del } \\
\text { modelo de migración }\end{array}$ & $\mathrm{R}$ & A & $\mathrm{A}, \mathrm{C}, \mathrm{F}$ & $\mathrm{V}$ & V \\
\hline 1.4 & Cierre & & & & & \\
\hline 1.4 .1 & Memoria del proyecto & $\mathrm{R}$ & A & $\mathrm{A}, \mathrm{C}, \mathrm{F}$ & $\mathrm{V}$ & V \\
\hline 1.4 .2 & $\begin{array}{l}\text { Cartera de proyectos } \\
\text { que asegure la } \\
\text { operatividad de la } \\
\text { plataforma migrada }\end{array}$ & $\mathrm{R}$ & A & $\mathrm{A}, \mathrm{C}, \mathrm{F}$ & $\mathrm{V}$ & V \\
\hline
\end{tabular}


Fuente: Elaboración Propia 


\section{PLAN DE GESTIÓN DE CALIDAD}

Se estableció un Plan de Gestión de Calidad para asegurar la calidad del proyecto durante todas sus fases, a continuación, se detallan algunos puntos descritos en el plan.

\section{Roles y Responsabilidades}

- Comité de Proyectos

- Aprobar proyectos profesionales

- Evaluar el avance del proyecto

- Decidir continuidad de proyectos

- Cliente

- Evaluar y dar seguimiento a los avances del proyecto

- Aprobar los resultados del proyecto

- Asesorar a los jefes de proyecto

- Gerente Profesor DATACENTER

- Dar seguimiento a los proyectos de la empresa

- Facilitar comunicación entre las empresas virtuales involucradas

- Aprobar los documentos de gestión

- Jefe de Proyecto

- Gestionar el proyecto

- Gestionar los recursos

- Definir el alcance del proyecto

- Coordinar con las autoridades involucradas en el proyecto

\section{Procedimientos}

- 1. Procedimiento para la Planificación de la Calidad 
- Definir aquellos entregables que deben pasar por el control de calidad, los responsables de este control y como se realizara dicho proceso.

- Definir aquellos certificados con los que debe contar el proyecto para validar que ha pasado por un proceso de control de calidad.

- Definir los recursos necesarios para las pruebas de control de calidad.

- Definir las fechas en el cronograma de cuando se realizarán estas pruebas de calidad.

- 2. Procedimiento para Aseguramiento de la Calidad

- Se realiza un monitoreo semanal de las fechas establecidas en el cronograma para asegurar el cumplimiento de las actividades.

- Asegurar la asignación de recursos de la empresa virtual QS para el aseguramiento de la calidad de los entregables.

- Evaluar el impacto de algún cambio dentro del proyecto (alcance, objetivos, tiempos, etc.) con respecto a la calidad.

\section{Procedimiento para el Control de la Calidad}

- El control de calidad se inicia revisando los entregables y la matriz de incidencias encontradas por el Analista de QS.

- Se analizan las mejoras y acciones correctivas para los entregables del proyecto. 


\section{PLAN DE GESTIÓN DE RIESGOS}

A continuación, se presenta un cuadro resumen de los riesgos establecidos para el proyecto y su análisis en relación a la probabilidad e impacto en el desarrollo del proyecto

Tabla 9: Gestión de Riesgos

\begin{tabular}{|c|c|c|c|c|c|c|c|c|c|}
\hline \multicolumn{5}{|c|}{ Identificación } & \multicolumn{5}{|l|}{ Análisis } \\
\hline $\begin{array}{l}\text { Cód. } \\
\text { Riesgo }\end{array}$ & $\begin{array}{l}\text { Fecha de } \\
\text { registro }\end{array}$ & $\begin{array}{l}\text { Categoría } \\
\text { - Origen }\end{array}$ & Descripción del Riesgo & Disparador & Probabilidad & Impacto (HH) & Impacto & $\begin{array}{l}\text { Exp al } \\
\text { Riesgo } \\
\text { (Horas) }\end{array}$ & $\begin{array}{l}\text { Exp al } \\
\text { Riesgo } \\
\text { (Costo } \\
\text { S/.) }\end{array}$ \\
\hline $\begin{array}{l}\text { MMNSDC- } \\
\text { R001 }\end{array}$ & $14 / 04 / 2017$ & $\begin{array}{l}\text { Ext - } \\
\text { Proveedor }\end{array}$ & $\begin{array}{l}\text { No contar con una señal de internet necesaria } \\
\text { para la implementación y validación y } \\
\text { validación del proyecto }\end{array}$ & $\begin{array}{l}\text { Incapacidad de migrar a la nube por } \\
\text { la velocidad de internet }\end{array}$ & $70 \%$-Alta & 20 & 4 Alta & 14.00 & 14 \\
\hline $\begin{array}{l}\text { MMNSDC- } \\
\text { R002 }\end{array}$ & $15 / 04 / 2017$ & $\begin{array}{l}\text { Ext } \\
\text { Usuarios }\end{array}$ & No contar con espacio en la nube a tiempo & $\begin{array}{l}\text { Incapacidad de migrar a la nube por } \\
\text { no contar con espacio en la nube }\end{array}$ & $70 \%$-Alta & 20 & 4 Alta & 14.00 & 14 \\
\hline
\end{tabular}




\begin{tabular}{|c|c|c|c|c|c|c|c|c|c|}
\hline $\begin{array}{l}\text { MMNSDC- } \\
\text { R003 }\end{array}$ & $16 / 04 / 2017$ & $\begin{array}{l}\text { Int }-\mathrm{El} \\
\text { proyecto }\end{array}$ & $\begin{array}{l}\text { No contar con la aprobación del cliente en } \\
\text { algún entregable }\end{array}$ & $\begin{array}{l}\text { Rechazo de algún entregable por } \\
\text { parte del cliente }\end{array}$ & $30 \%$ Baja & 20 & 4 Alta & 6.00 & 6 \\
\hline $\begin{array}{l}\text { MMNSDC- } \\
\text { R004 }\end{array}$ & $17 / 04 / 2017$ & $\begin{array}{l}\text { Int } \\
\text { Equipo de } \\
\text { trabajo }\end{array}$ & Estimación errónea de tiempo & Retraso en alguna actividad & $50 \%$ Media & 20 & 4 Alta & 10.00 & 10 \\
\hline $\begin{array}{l}\text { MMNSDC- } \\
\text { R005 }\end{array}$ & $18 / 04 / 2017$ & $\begin{array}{l}\text { Ext }- \\
\text { Usuarios }\end{array}$ & $\begin{array}{l}\text { Cambios en el calendario del Comité de } \\
\text { Proyectos. }\end{array}$ & $\begin{array}{l}\text { Informe de cambio de fechas por } \\
\text { parte del comité de proyectos }\end{array}$ & $10 \%$ Muy baja & 20 & 4 Alta & 2.00 & 2 \\
\hline $\begin{array}{l}\text { MMNSDC- } \\
\text { R006 }\end{array}$ & $19 / 04 / 2017$ & $\begin{array}{l}\text { Ext }- \\
\text { Usuarios }\end{array}$ & $\begin{array}{l}\text { Modificación del alcance por el Comité de } \\
\text { Proyectos. }\end{array}$ & Cambio de alcance por el comité & $30 \%$ Baja & 20 & 4 Alta & 6.00 & 6 \\
\hline $\begin{array}{l}\text { MMNSDC- } \\
\text { R007 }\end{array}$ & $20 / 04 / 2017$ & $\begin{array}{l}\text { Ext }- \\
\text { Usuarios }\end{array}$ & $\begin{array}{l}\text { No conseguir financiación por parte de } \\
\text { DATACENTER para la validación del } \\
\text { modelo. }\end{array}$ & No obtener financiación de la UPC & $70 \%$-Alta & 20 & 4 Alta & 14.00 & 14 \\
\hline
\end{tabular}

\section{Fuente: Elaboración Propia}

El siguiente cuadro representa las estrategias de respuesta y monitoreo de los riesgos antes presentados.

Tabla 10: Estrategias de Respuesta 


\begin{tabular}{|c|c|c|c|c|c|c|c|c|c|}
\hline \multirow{2}{*}{$\begin{array}{l}\text { Identificación } \\
\text { Cód. Riesgo }\end{array}$} & \multicolumn{7}{|c|}{ Estrategia de respuesta } & \multicolumn{2}{|l|}{ Monitoreo } \\
\hline & $\begin{array}{l}\text { Tipo de } \\
\text { Estrategia }\end{array}$ & $\begin{array}{l}\text { Descripción de la } \\
\text { estrategia planteada }\end{array}$ & $\begin{array}{l}\text { Rol } \\
\text { responsable }\end{array}$ & $\begin{array}{l}\text { Nombre } \\
\text { Responsable }\end{array}$ & $\begin{array}{l}\text { Acciones } \\
\text { realizadas }\end{array}$ & $\begin{array}{l}\text { Situación de la } \\
\text { estrategia }\end{array}$ & $\begin{array}{l}\text { Plan de } \\
\text { Contingencia }\end{array}$ & Estado & $\begin{array}{ll}\text { Fecha de } & \text { de } \\
\text { cambio } & \text { de } \\
\text { estado } & \end{array}$ \\
\hline MMNSDC-R001 & Mitigar & $\begin{array}{l}\text { Solicitar } \\
\text { anticipadamente a los } \\
\text { encargados } \\
\text { DATACENTER de } \\
\text { disponer de una buena } \\
\text { conexión a internet }\end{array}$ & Jefe & $\begin{array}{l}\text { Fabiola Loo / } \\
\text { Christian Rojas }\end{array}$ & $\begin{array}{l}\text { Se informará que el } \\
\text { modelo no se } \\
\text { llegará a validar }\end{array}$ & No iniciado & 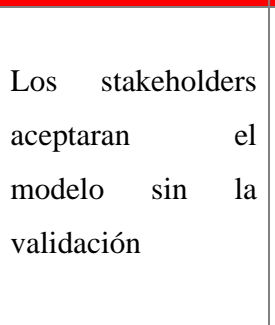 & No Incurrido & N/A \\
\hline MMNSDC-R002 & Mitigar & $\begin{array}{l}\text { Solicitar al profesor } \\
\text { gerente anticipadamente } \\
\text { que se nos brinde un } \\
\text { espacio en la nube para la } \\
\text { implementación y } \\
\text { validación. }\end{array}$ & Jefe & $\begin{array}{l}\text { Fabiola Loo / } \\
\text { Christian Rojas }\end{array}$ & $\begin{array}{l}\text { Crear una cuenta } \\
\text { gratuito de prueba }\end{array}$ & No iniciado & $\begin{array}{l}\text { Se hará uso de una } \\
\text { cuenta de prueba de } \\
\text { estudiante para la } \\
\text { validación, y solo } \\
\text { se usará hasta el } \\
\text { límite de la misma } \\
\text { (sin incurrir en } \\
\text { gastos adicionales). }\end{array}$ & No Incurrido & N/A \\
\hline MMNSDC-R003 & Mitigar & $\begin{array}{l}\text { Coordinar reuniones } \\
\text { periódicas con el cliente } \\
\text { a fin de evaluar el } \\
\text { avance. }\end{array}$ & Jefe & $\begin{array}{l}\text { Fabiola Loo / } \\
\text { Christian Rojas }\end{array}$ & $\begin{array}{l}\text { Proponer nueva } \\
\text { fecha de entrega }\end{array}$ & No iniciado & $\begin{array}{lr}\text { El } & \text { equipo } \\
\text { propondrá } & \text { una } \\
\text { nueva fecha } & \text { de } \\
\text { entrega para } & \text { la } \\
\text { corrección. } & \text { Serán }\end{array}$ & No Incurrido & N/A \\
\hline
\end{tabular}




\begin{tabular}{|c|c|c|c|c|c|c|c|c|c|}
\hline & & & & & & & \begin{tabular}{|ll} 
horas de trabajo \\
adicionales que no \\
comprometan el \\
desarrollo & \\
continuo. &
\end{tabular} & & \\
\hline MMNSDC-R004 & Mitigar & $\begin{array}{l}\text { Realizar seguimiento } \\
\text { constante al cronograma } \\
\text { a fin de realizar cambios } \\
\text { necesarios. }\end{array}$ & Jefe & $\begin{array}{l}\text { Fabiola Loo / } \\
\text { Christian Rojas }\end{array}$ & $\begin{array}{l}\text { Informar el retraso } \\
\text { al cliente }\end{array}$ & No iniciado & $\begin{array}{llr}\text { El } & \text { equipo } \\
\text { informará } & \text { sobre } & \text { el } \\
\text { retraso } & \text { de } & \text { un } \\
\text { entregable } & \text { en } & \text { las } \\
\text { reuniones } & \text { para } \\
\text { reorganizar } & \text { los } \\
\text { siguientes } & \text { y } & \text { no } \\
\text { cometer } & \text { el } & \text { mismo } \\
\text { error. } & & \end{array}$ & No Incurrido & N/A \\
\hline MMNSDC-R005 & Mitigar & $\begin{array}{l}\text { Coordinar con el gerente } \\
\text { profesor para tomar las } \\
\text { medidas necesarias si en } \\
\text { caso perjudique de } \\
\text { manera directa a nuestro } \\
\text { proyecto. }\end{array}$ & Jefe & $\begin{array}{l}\text { Fabiola Loo / } \\
\text { Christian Rojas }\end{array}$ & $\begin{array}{l}\text { Coordinar con el } \\
\text { gerente profesor }\end{array}$ & No iniciado & $\begin{array}{l}\text { Coordinar con el } \\
\text { gerente profesor } \\
\text { para tomar las } \\
\text { medidas necesarias } \\
\text { si en caso } \\
\text { perjudique de } \\
\text { manera directa a } \\
\text { nuestro proyecto. }\end{array}$ & No Incurrido & N/A \\
\hline
\end{tabular}




\begin{tabular}{|c|c|c|c|c|c|c|c|c|c|}
\hline MMNSDC-R006 & Mitigar & $\begin{array}{l}\text { Modificar todos los } \\
\text { documentos de gestión y } \\
\text { la aprobación del gerente } \\
\text { profesor. }\end{array}$ & Jefe & $\begin{array}{l}\text { Fabiola Loo / } \\
\text { Christian Rojas }\end{array}$ & $\begin{array}{l}\text { Actualizar los } \\
\text { documentos } \\
\text { correspondientes }\end{array}$ & No iniciado & \begin{tabular}{|lr} 
Modificar todos los \\
documentos & de \\
gestión y & la \\
aprobación & del \\
gerente profesor.
\end{tabular} & No Incurrido & N/A \\
\hline MMNSDC-R007 & Mitigar & $\begin{array}{l}\text { Solicitar la financiación } \\
\text { con anticipación a la } \\
\text { DATACENTER. }\end{array}$ & Jefe & $\begin{array}{l}\text { Fabiola Loo / } \\
\text { Christian Rojas }\end{array}$ & $\begin{array}{l}\text { Crear una cuenta } \\
\text { gratuito de prueba }\end{array}$ & No iniciado & $\begin{array}{l}\text { Se hará uso de una } \\
\text { cuenta de prueba de } \\
\text { estudiante para la } \\
\text { validación, y solo } \\
\text { se usará hasta el } \\
\text { límite de la misma } \\
\text { (sin incurrir en } \\
\text { gastos adicionales). }\end{array}$ & No Incurrido & N/A \\
\hline
\end{tabular}

Fuente: Elaboración Propia 


\section{CONCLUSIONES}

- Se realizó la investigación, en la cual se encontró otras metodologías para la migración hacia la nube de la infraestructura, estos abarcaron requerimientos, proveedores y hasta fases, pero eran independientes entre ellos. Se hizo una comparativa, según factores obtenidos de la literatura. Como resultado se obtuvo que no todos los modelos cumplen con todos los factores.

- Se realizó el juicio experto por parte de un especialista en cloud, por el método de NPS (Net Promoter Score). El cual tuvo una puntuación del 94\%, considerándose aprobada.

- Se realizó la validación del modelo mediante un caso de uso, en una empresa del sector de servicios financieros, de dicha validación se obtuvieron los siguientes resultados:

- Se pudo concluir por medio de un análisis que se ha reducido hasta en un $45 \%$ los riesgos existentes durante la migración.

- Luego de haber analizado los costos del proyecto de migración y los beneficios, se calcula un ROI mensual de $19,77 \%$, siendo total de meses para recuperar la inversión de 5.06 meses.

- Se analizó el alineamiento entre los objetivos del proyecto y los objetivos de la empresa, dando como resultado que todos los objetivos del proyecto están relacionados con al menos un objetivo del negocio.

- Se desarrollo un plan de continuidad, el cual está basado en: Capacitaciones, SLAs, Back ups - DRP Cloud y Soporte.

- Se realizó un plan de soporte, el cual tiene tareas que permiten a la empresa continuar con sus actividades en caso haya un incidente. Estos fueron basados en estándares de aceptación mundial (ITIL). 


\section{RECOMENDACIONES}

- Se recomienda investigar sobre la facturación de los servicios cloud que se utilizaran, esto debido que, a pesar de contar con capas o servicios de prueba para experimentos, estas pueden exceder los límites, lo que ocasionaría pagos por la diferencia del total usado menos lo cubierto por la plataforma.

- Se recomienda contar con herramientas alternativas en caso sea necesario, debido a que se puede dar el caso que algunas políticas de estas cambien e impacten negativamente al proyecto.

- Se recomienda que la etapa de investigación incluya sobre las plataformas que se podrían utilizar, esto para evitar temas de incompatibilidades técnicas en el futuro.

- Se recomienda explicar los beneficios a la organización (y traducirlo en términos monetarios) y dejarlo en claro desde el principio para evitar confusiones en el futuro.

- Se recomienda realizar la migración de los servidores virtuales en un horario que no afecte las operaciones del negocio, en caso no sea posible se recomienda que sea en el que menor impacto ocasione.

- Se recomienda contar con un ancho de banda considerable, pues el migrar a la nube puede conllevar a subir varios gigabytes de información a cloud.

- Se recomienda segmentar las cargas de trabajo a fin de evitar saturar el enlace de conexión. 


\section{GLOSARIO}

- Alcance: Indicador de hasta donde abarca el proyecto.

- Arquitectura: Organización fundamental de un sistema, representada por sus componentes, sus relaciones entre ellos y con su entorno, y los principios que gobiernan su diseño y evolución.

- Benchmarking: Evaluación comparativa contra los competidores más fuertes de un rubro.

- CCRA: Cloud Computing Reference Architecture.

- Cloud Computing: Modelo de entrega de servicios a través de internet.

- Data Center: Centro de procesamiento de datos, conformada por un conjunto de componentes asociados como servidores, switches, routers, entre otros.

- EDT: Estructura de descomposición de trabajo.

- Hipervisor: Plataforma que permite aplicar diversas técnicas de control de virtualización.

- IaaS: Infrastructure as a Service.

- Imagen: Punto de referencia de una máquina virtual, el cual puede ser replicado en diversas instancias.

- Instancia: Particularización o representación especifica de una determinada imagen.

- Migración: Acción de mover desde un punto de origen a un punto de destino.

- PaaS: Plataform as a Service.

- PMBOK: Project Management Body of Knowledge.

- PYME: Pequeña y Mediana Empresa.

- ROI: Return on Invesment.

- Saas: Software as a Service.

- Servidor On-Demand: Servidor que se encuentra en una plataforma cloud y es usado por el cliente 
- Servidor On-Premise; Servidor que se encuentra implementados físicamente en el lado del usuario..

- Solución: Respuesta frente a un problema identificado.

- Stakeholders: Personas u organizaciones interesadas en el proyecto.

- Bucket: Storage o repositorio de almacenamiento de información en AWS.

- Student Outcomes: Puntos que debe cumplir el perfil del egresado de la carrera de Ingeniería de Sistemas de Información de la UPC.

- TI: Tecnologías de Información.

- TOGAF: The Open Group Architecture Framework.

\section{BIBLIOGRAFÍA}

A. Baruchi, E. T. (2015). Reducing Virtual Machine Live Migration Overhead via Workload Analysis. IEEE Latin America Transactions.

Alibaba. (s.f.). Alibaba Cloud. Recuperado el 28 de Setiembre de 2017, de Alibaba Cloud: https://www.alibabacloud.com/solutions/hybrid?spm=a3c0i.7911826.675768.dnavsoluti ons7.d68eb3fai3e4y

Amazon Web Services. (Diciembre de 2015). A Practical Guide to Cloud Migration. Obtenido de Amazon Web Services: https://d0.awsstatic.com/whitepapers/the-path-tothe-cloud-dec2015.pdf

Amazon. (s.f.). Amazon Web Services. Recuperado el 15 de Junio de 2017, de Amazon Web Services: https://aws.amazon.com/es/what-is-aws/

Artaza, S. d. (31 de Octubre de 2016). MuyCloud. Obtenido de Trece buenas prácticas para una migración de éxito a la nube: http://muycloud.com/2016/10/31/buenaspracticas-migracion-nube/

Erber, M. (30 de Enero de 2014). What is hybrid cloud? Obtenido de https://www.ibm.com/blogs/cloud-computing/2014/01/what-is-hybrid-cloud/

Fourcade, G. (7 de Julio de 2016). IBM. Obtenido de Seis variable para analizar antes de subir a la nube: https://www.ibm.com/ar/services/pdf/final_seis_variables_para_analizar_antes_de_salta r_a_la_nube.pdf 
Gartner. (2 de Julio de 2017). Gartner. Obtenido de Cloud Computing: http://www.gartner.com/it-glossary/Cloud-computing

Google. (s.f.). Google Cloud Platform. Recuperado el 14 de Junio de 2017, de Google Cloud Platform: https://cloud.google.com/?hl=es

Hamid Reza Bazi, A. H. (2017). A comprenhesive framework for cloud computing migration using Meta-Synthesis approach. Journal of Systems and Software.

Hewlett Packard Enterprise. (2016). HP. Obtenido de Migración de aplicaciones a la nube local: https://h20195.www2.hpe.com/V2/getpdf.aspx/4AA6-3932ESE.pdf?ver=1.0

IBM. (2014). Seis variables para analizar antes de saltar a la nube. IBM.

IBM. (s.f.). Cloud Computing: Bluemix. Obtenido de Bluemix: https://www.ibm.com/cloud-computing/bluemix/es

J. Hwang, K. B. (2016). Automation and orchestration framework for large-scale enterprise cloud migration. IBM Journal of Research and Development.

Mahdi Fahmideh Gholami, F. D. (2016). Cloud migration process-A survey, evaluation framework, and open challenges. Journal of Systems and Software.

Michael Menzel, R. R. (2014). CloudGenius: A Hybrid Decision Support Method for Automating the Migration of Web Application Clusters to Public Clouds. IEEE Transactions on Computers.

Microsoft. (2 de Julio de 2017). Azure. Obtenido de ¿Qué es IaaS?: https://azure.microsoft.com/es-es/overview/what-is-iaas/

Microsoft. (2 de Julio de 2017). Azure. Obtenido de ¿Qué es PaaS?: https://azure.microsoft.com/es-es/overview/what-is-paas/

Microsoft. (02 de Julio de 2017). Azure. Obtenido de ¿Qué es SaaS?: https://azure.microsoft.com/es-es/overview/what-is-saas/

Microsoft. (s.f.). Microsoft Azure. Recuperado el 14 de Junio de 2017, de Microsoft Azure: https://azure.microsoft.com/es-es/

Oracle. (s.f.). Oracle Cloud. Recuperado el 28 de Setiembre de 2017, de Oracle Cloud: https://cloud.oracle.com/en_US/iaas

Panetta, K. (21 de Abril de 2017). Gartner. Obtenido de 3 Journeys for Migrating a Data Center to Cloud IaaS : https://www.gartner.com/smarterwithgartner/3-journeys-formigrating-a-data-center-to-cloud-iaas/

Pooyan Jamshidi, A. A. (2014). Cloud Migration Research: A Systematic Review. IEEE Transactions on Cloud Computing. 
PowerData. (10 de Junio de 2015). PowerData. Obtenido de Plan de migración de datos: elementos imprescindibles: http://blog.powerdata.es/el-valor-de-la-gestion-dedatos/bid/397552/plan-de-migraci-n-de-datos-elementos-imprescindibles

Ramirez-Nafarrate, J. O.-G. (2015). Collaborative Agents for Distributed Load Management in Cloud Data Centers using Live Migration of Virtual Machines. IEEE Transactions on Services Computing.

Red Hat. (2016). Red Hat Consulting. Obtenido de Migraciones en la nube: https://www.redhat.com/cms/managed-files/co-cloudmigrations-datasheetinc03702101w-201603-a4-es.pdf

Rouse, M. (Agosto de 2017). Search Cloud Computing. Obtenido de GCP: http://searchcloudcomputing.techtarget.com/definition/Google-Cloud-Platform

Sosa, L. V. (7 de Agosto de 2012). Almacenamiento en las nubes Híbridas. Obtenido de http://ingenierosoym.blogspot.pe/

Talent Fifty Plus. (s.f.). Talent Fifty Plus. Obtenido de Transicion a la nube para PYMES: http://www.talentfiftyplus.es/descargas/Trans_Cloud.pdf

TIC Portal. (2017). TIC Portal. Obtenido de Amazon Web Services: https://www.ticportal.es/temas/cloud-computing/amazon-web-services

Weiwei Qiu, Z. Z. (2014). Reliability-Based Design Optimization for Cloud Migration. IEEE Transactions on Services Computing.

\section{ANEXOS}




\section{ANEXO 1: PLANTILLA PRINCIPIOS, OBJETIVOS Y MOTIVADORES DEL NEGOCIO}

1. Propósito del documento

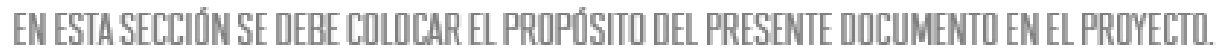

2. Principios del negocio

EN ESTA SECCIÓN DE COLDCARAN LOS PRINCIPIOS DEL NEGOCIO

3. Objetivos del negocio

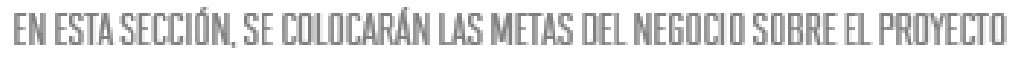

4. Motivadores del negocio

EN ESTA SECCIÓN, SE COLOCARAN LOS BUSINESS DRIVER, KPI'S o FACTORES CRITIICOS QUE TIENE El NEGOCIO SOBREE PROYECTO. 


\section{ANEXO 2: PLANTILLA LEVANTAMIENTO DE}

1. Inventario de Servidores:

\begin{tabular}{|c|c|c|c|c|c|c|}
\hline Servidor Flsico & Caracteristicas & Servicios & Descripción & $\begin{array}{l}\text { Servidor } \\
\text { Virtual }\end{array}$ & $\begin{array}{l}\text { Caracteristicas } \\
\text { Servidor Virtual }\end{array}$ & Involucrados \\
\hline $\begin{array}{c}\text { [Se dehe } \\
\text { detallar segón } \\
\text { el usuario }\end{array}$ & $\begin{array}{l}\text { [Se dehe detallar } \\
\text { según el usuario] }\end{array}$ & $\begin{array}{l}\text { [Se debe } \\
\text { detallar según } \\
\text { el usuario] }\end{array}$ & $\begin{array}{l}\text { [Se dehe detallar según el } \\
\text { usuario] }\end{array}$ & $\begin{array}{l}\text { [Se debe } \\
\text { detallar } \\
\text { según el } \\
\text { usuario] }\end{array}$ & $\begin{array}{c}\text { [Se debe } \\
\text { detallar según el } \\
\text { usuario] }\end{array}$ & $\begin{array}{l}\text { [Se debe } \\
\text { detallar según } \\
\text { el usuario] }\end{array}$ \\
\hline
\end{tabular}

2. Diagrama de Infraestructura:

2.1. Infraestructura fisica:

[Se debe detallar la infraestructura fisica actual del usuario]

2.2. Infraestructura con Software Base

[Se debe detallar la infraestructura con sw base actual del usuario]

2.3. Virtualización

[Se debe detallar la virtualización actual del usuario]

2.4. Servicios

2.4.1. Vista general:

[Se debe detallar la vista general de los servicios actuales]

3. Configuración de Firewall

[Se debe detallar la configuración del firewall actual]

\section{INFORMACIÓN}




\section{ANEXO 3: PLANTILLA ESPECIFICACIÓN DE}

\section{Datos fenerales del Proyecto}

Nombre del Proyecto:

[Se detalla el nombre del prayecto]

\begin{tabular}{|c|c|}
\hline Liter Ustario & Especialista Cloud \\
\hline [Nombre de Usuario] & [Nombre del Especialista] \\
\hline Fecha de Inicio & Fechade dorrobación \\
\hline [FECHA DE INICIO] & [FECHA DEAPROBACION] \\
\hline
\end{tabular}

1. Antecerlentes

[Se detalla las antecedentes de la empresa]

2. Objetivo dal Requriniento

[Se detalla los objetivos del proyecto]

3. Descripaión del Requerimiento

3.1. Alcance Funcional

[Se detalla cual será el alcance funcional del proyecto]

3.2. Requerimientos Funcianales

[Se detalla cual será el Requerimientas Funcionales]

3.3. Requerimientos Na Funcionales

[Se detalla cual será el Requerimientus No Funcionales]

3.4. Exclusiones (a ser llenado por el Especialista)

[Se detalla cual será las exclusiones]]

4. Aleance Técanico

[Se detalla el alcance Técnico]

5. Consideraciones

- Cualquier cambio en el alcance descrito durante el desarrollo será evaluado como un control de cambio o una mejara adicional que deberá aprobarse por el usuario previamente para ser planificada.

\section{REQUERIMIENTOS}




\section{ANEXO 4: PLANTILLA BENCHMARKING}

1. Descripción del Activo Preexistente

[Se detalla el inventario preexistente de lo que se va a evaluar (Hardware, Plataforma, Software, etc.)]

2. Alternativas a considerar

[Se detallar las ufertas del mercado a considerar en la evaluación]

3. Análisis Comparativo

a) Propósito de evaluación

[Se detalla el propísito de la evaluación Benchmarking]

b) Modelo de calidad

a. Para establecer el Modelo de Calidad a utilizar dentro de la evaluación se consideró la ISO/IEC 25000, conocida como SQuaRE (System and Software Quality Requirements and Evaluation). que tiene por abjetiva la creación de un marco de trabajo camún para evaluar la calidad del producto software.

b. En base al modelo de calidad para la calidad externa e interna se establecieron los atributos a valurar para el análisis de la herramienta a escoger:

- Funcionalidad

- Fiabilidad

- Usabilidad

- Eficiencia

- Mantenimiento

- Portabilidad

- Eficacia

- Productividad

- Satisfacción

- Seguridad

c) Métricas

\begin{tabular}{|l|l|l|}
\hline \multicolumn{1}{|c|}{ Rriterios de calficicación } & \multicolumn{1}{c|}{ Escala de puntuación } & \multicolumn{1}{c|}{ Puntuación } \\
\hline Cumple totalmente & Avanzado & 80 a 100 \\
\hline Cumple parcialmente & Intermedio & 40 a BD \\
\hline Cumple mInimo & Básico & 20 a 40 \\
\hline
\end{tabular}

Puntaje mlnimo de aceptación

[Se dehe definir con el usuario Ider, cuál serla el puntaje mlnimo] 
d) Evaluación técnica

Cuadro I: Evaluación técnica

\begin{tabular}{|c|c|c|c|c|}
\hline & ilbutos & \multicolumn{2}{|c|}{ Descripción } & Puntaje \\
\hline 1 & \multicolumn{3}{|c|}{ Calidad intema y Externa } & \\
\hline 1.1 & Funcionalidad & \multicolumn{2}{|c|}{$\begin{array}{l}\text { Cumplimiento de las funcianes principales al 100\% de } \\
\text { administrar servidores, memoriay yedes, y compatibilidad con } \\
\text { sistemas operativos, etc. }\end{array}$} & $\begin{array}{c}\text { [Puntaje a definir par el } \\
\text { usuario] }\end{array}$ \\
\hline 1.2 & Fiabilidad & \multicolumn{2}{|c|}{$\begin{array}{l}\text { Capacidad para mantener un nivel especificado de análisis y } \\
\text { solución, en caso de errores. }\end{array}$} & $\begin{array}{c}\text { [Puntaje a definir por el } \\
\text { usuario] } \\
\end{array}$ \\
\hline 1.3 & Usabilidad & \multicolumn{2}{|c|}{ Facilidad, rapidez de camprensián a nivel usuario y técnico. } & $\begin{array}{c}\text { [Puntaje a definir por el } \\
\text { usuario] } \\
\end{array}$ \\
\hline \multirow[t]{2}{*}{1.4} & \multirow[t]{2}{*}{ Eficiencia } & Comportamiento & $\begin{array}{l}\text { Alto rendimiento para análisis y } \\
\text { procesos de datos. }\end{array}$ & $\begin{array}{c}\text { PPuntaje a definir por el } \\
\text { usuario] }\end{array}$ \\
\hline & & Utilización de recursos & $\begin{array}{l}\text { El software debe consumir } \\
\text { recursos mlnimos de la PC, sin } \\
\text { afectar capacidad multitarea del } \\
\text { procesaddor vs memoria. }\end{array}$ & $\begin{array}{c}\text { [Puntaje a definir par el } \\
\text { usuario] }\end{array}$ \\
\hline \multirow[t]{2}{*}{1.5} & \multirow[t]{2}{*}{ Mantenimiento } & Actualización & $\begin{array}{l}\text { Facilidad para actualizar de } \\
\text { manera manual o automática. }\end{array}$ & $\begin{array}{c}\text { [Puntaje a definir par el } \\
\text { usuario] }\end{array}$ \\
\hline & & Soparte & Servicio técnico local & $\begin{array}{c}\text { [Puntaje a definir par el } \\
\text { usuario] }\end{array}$ \\
\hline \multirow[t]{2}{*}{1.6} & \multirow[t]{2}{*}{ Portabilidad } & Adaptabilidad & $\begin{array}{l}\text { Adaptable a distintos entornos de } \\
\text { software. }\end{array}$ & $\begin{array}{c}\text { Pruntaje a definir por el } \\
\text { usuario] }\end{array}$ \\
\hline & & Facilidad de instalación & $\begin{array}{l}\text { Contar can asistente de } \\
\text { instalación. }\end{array}$ & $\begin{array}{l}\text { PPuntaje a definir por el } \\
\text { usuario] }\end{array}$ \\
\hline 2 & \multicolumn{3}{|c|}{ Calidad de Uso } & \\
\hline 2.1 & Eficacia & \multicolumn{2}{|c|}{$\begin{array}{l}\text { Capacidad de alcanzar las metas operativas al } 100 \% \text { al } \\
\text { momento del uso de las funciones de administración de } \\
\text { servidores, memoria y redes por el usuario. }\end{array}$} & $\begin{array}{c}\text { [Puntaje a definir par el } \\
\text { usuario] }\end{array}$ \\
\hline 2.2 & Seguridad & \multicolumn{2}{|c|}{$\begin{array}{l}\text { Capacidad de ser confiabley seguro para la información que se } \\
\text { administre con dicha herramienta. }\end{array}$} & $\begin{array}{l}\text { PPuntaje a definir por el } \\
\text { usuario] }\end{array}$ \\
\hline 2.3 & Satisfacción & \multicolumn{2}{|c|}{$\begin{array}{l}\text { Capacidad para cubrir sus requerimientos con total } \\
\text { conformidad del usuaria. }\end{array}$} & $\begin{array}{l}\text { Pruntaje a definir por el } \\
\text { usuario] }\end{array}$ \\
\hline 2.4 & Productividad & \multicolumn{2}{|c|}{$\begin{array}{l}\text { No deberá de cansumir muchos recursos de memoria y } \\
\text { procesadar en los equipos donde se hayan instalada. }\end{array}$} & $\begin{array}{c}\text { Pruntaje a definir por el } \\
\text { usuario] }\end{array}$ \\
\hline
\end{tabular}

4. Conclusiones

[Se debe definir las conclusiones] 
ANEXO 5: PLANTILLA DE ESTUDIO DE

\section{ESTUDIO DE VIABILIDAD TECNICA}

a. DESARROLLO

Para realizar la viabilidad técnica, se debe completar el archivo Viabilidad_ Tecnicaxls

b. CONCLUSION

Se coloca la conclusión del desarrollo de la viabilidad técnica

\section{ESTUDIO DE VIABILIDAD ECONOMICA}

a. DESARROLLO

Para realizar la viabilidad económica, se debe completar el archivo Viabilidad_Economicaxls

b. BENEFICIOS

Se debe colocar los beneficios encontrados en la Viabilidad_Economicaxls

c. CONCLUSION

Se coloca la conclusión del desarrollo de la viabilidad técnica

\section{$\triangle$ 3. ESTUDIO DE VIABILIDAD OPERATIVA}

a. CONCLUSION

Se coloca la conclusión del desarrollo de la viabilidad.

4. CONCLUSION DE LA VIABILIDAD

\section{VIABILIDAD Y HERRAMIENTA}


HeRramienta: ViabiliddAD TECNICA

\begin{tabular}{|c|c|c|c|c|c|c|}
\hline Numero & Servidor & Caracteristicas & Valor & Dificultad & $\begin{array}{l}\text { Dificultad } \\
\text { Acumulada }\end{array}$ & Resultado \\
\hline \multirow{5}{*}{1} & \multirow{5}{*}{ BD } & Sistema Operativo & & $\# N / A$ & \multirow{5}{*}{$\# N / A$} & \\
\hline & & Disco Duro Ocupado(GB) & & 0 & & \\
\hline & & Formato Disco Duro & & $\# N / A$ & & \\
\hline & & Servidor Virtualizado & & $\# N / A$ & & \\
\hline & & $\begin{array}{l}\text { Contiene cargas de trabajo relacionado } \\
\text { a regulación sensible }\end{array}$ & & $\# N / A$ & & \\
\hline
\end{tabular}

\begin{tabular}{|l|l|}
\hline Rango & Resultado \\
\hline$[1 ; 5>$ & Migración Sencilla \\
\hline$[5 ; 9>$ & Migración Compleja Factible \\
\hline & \\
{$[9 ; 14>$} & Migración Muy Compleja \\
\hline$[14 ;+\infty>$ & Migración no Factible \\
\hline
\end{tabular}




\section{HERRAMIENTA: VIABILIDAD ECONOMICA}

COSTOS DE SOFIWARE HARDWARE Y PERSONAL

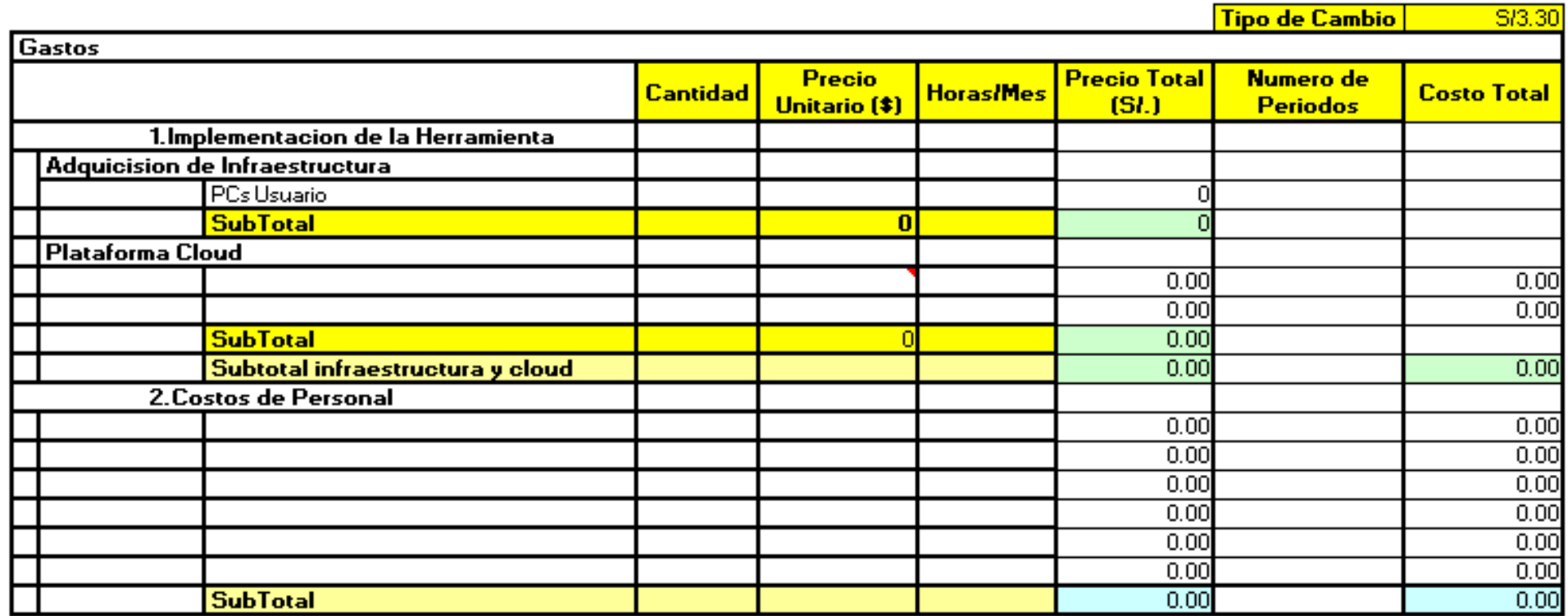

\begin{tabular}{|c|c|c|c|c|c|}
\hline GASTOS DE MANIENIMIENTO & MEIRICA & COSTO UNIIARIO & COSIO MENSUALL & COSIO ANUALL & \\
\hline & & & S/. & $\mathrm{S} /$. & $\cdot$ \\
\hline & & & S/. & $\mathrm{s} /$. & $\cdot$ \\
\hline & & & $\$ /$. & $\$ /$. & - \\
\hline \multirow[t]{2}{*}{ COSTOS DE PERSONAL } & MEIRICA & COSIO UNIIARIO & COSTO MENSUAL L & COSIO ANUAL & \\
\hline & & & S/. & S/. & - \\
\hline & & TOTAL & $S /$. & $S /$. & - \\
\hline
\end{tabular}

\begin{tabular}{|l|r|c|c|}
\cline { 3 - 4 } \multicolumn{2}{c|}{} & ROI MENSUAL & ROI ANUAL \\
\hline INYERSIŌN & S/COO & \#IDIV/0! & \#IDIV/0! \\
\hline
\end{tabular}




\begin{tabular}{|c|c|c|}
\hline \multicolumn{2}{|c|}{ Mes } & Ganancias \\
\hline \multirow{3}{*}{ 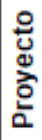 } & 1 & S/. 0.00 \\
\hline & 2 & SI. 0.00 \\
\hline & 3 & SI. 0.00 \\
\hline \multirow{15}{*}{ 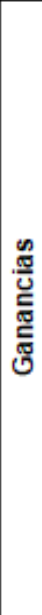 } & 4 & \#; DIVIO! \\
\hline & 5 & \#[D|V/O! \\
\hline & 6 & \#[DIVIO! \\
\hline & 7 & \#[DIV/0! \\
\hline & 8 & \#:D|VIO! \\
\hline & 9 & \#[DIVIO! \\
\hline & 10 & \#[DIVI0! \\
\hline & 11 & \#; [D|V/O! ! \\
\hline & 12 & \#;DIVIO! \\
\hline & 13 & \#[DIVIO! \\
\hline & 14 & \#:[D|vio! \\
\hline & 15 & \#;[D|vio! \\
\hline & 16 & \#;[D|vio! \\
\hline & 17 & \#;[IVIO! \\
\hline & 18 & $\#_{;} \mathrm{DIV} / 0$ ! \\
\hline
\end{tabular}

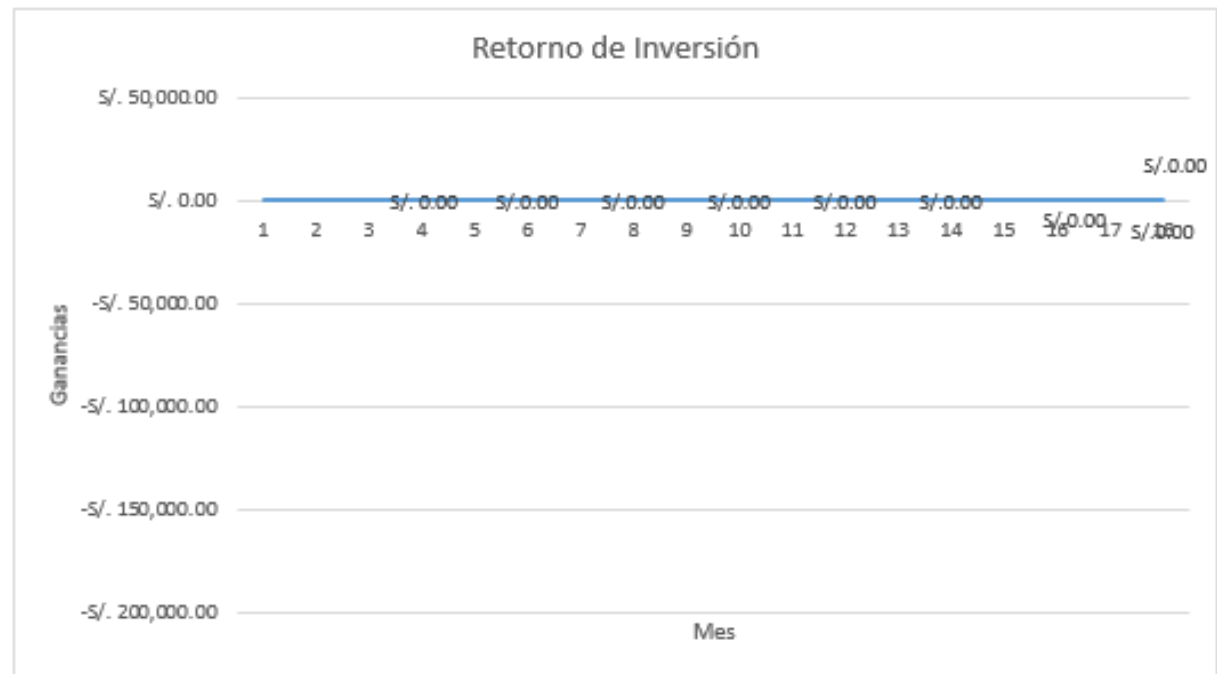

\section{ANEXO 6: PLANTILLA DE DOCUMENTO DE IMPLEMENTACIÓN}

l. PROPÓSITO DEL DOCUMENTO

[Se explica el propósito del presente documento]

2. PROCESO DE MIGRACIÓN

[Se detalla el proceso de la migración paso a paso]

\section{CONCLUSIONES}

[Se explican las conclusiones del proyecto]

\section{ANEXO 7: PRINCIPIOS, OBJETIVOS Y MOTIVADORES DE NEGOCIO}


1. Propósito del documento

- En el presente documento se definirán las principios, metas/objetivos y factores del proyecto en el negocio de Globokas Perú.

2. Principios del neǵocio

- Continuidad de negocio

- Alcanzar los objetivos

- Maximizar los beneficios de la arganización

- Los datos san un activo

3. Objetivos del neǵocio

- Superar a la competencia en visibilidad y venta dentro del mercado.

- Incursionar las ventas an-line con un margen de éxito sostenible.

- Reducir al minima los gastos y recordar el déficit en al menos $40 \%$

- Ser una marca que los demandantes reconozcan par su innovación.

- Mejorar la rentabilidad de la arģanización en un 3\% en el año 2018.

4. Motivadores del neǵocio

- Reducir costos de Ti

- Optimizar el centro de datos

- Disponibilidad optimizada de los servicios criticos

- Creación de una organización más ágil basada en la automatización de su DC.

- Ventaja Competitiva

- Integración de sus servicios de TI(Almacenamiento, servidares, redes, energla, interoperabilidad) 


\section{ANEXO 8: LEVANTAMIENTO DE INFORMACIÓN}

\begin{tabular}{|c|c|c|c|c|c|c|}
\hline $\begin{array}{l}\text { Servidor } \\
\text { Flsico }\end{array}$ & Caracteristicas & Servicios & Descripción & $\begin{array}{c}\text { Servidor } \\
\text { Virtual }\end{array}$ & $\begin{array}{l}\text { Caracteristicas } \\
\text { Servidor Virtual }\end{array}$ & Involucrados \\
\hline GKDCOI & $\begin{array}{l}\text { Procesador: Intel Core } \\
\text { í } 2.06 \mathrm{~Hz} \\
\text { Disco Duro: } 320 \text { 6B } \\
\text { Memoria RaM: } 368 \\
\text { Tarjeta de Red: Gigahit } \\
\text { Ethernet } \\
\text { Sistema Operativo: } \\
\text { linux }\end{array}$ & $\begin{array}{l}\text { Servidor } \\
\text { Domain }\end{array}$ & $\begin{array}{l}\text { Contiene el } \\
\text { Domain Controller }\end{array}$ & No aplica & No aplica & TI \\
\hline \multirow[t]{2}{*}{ GKSVor } & $\begin{array}{l}\text { Procesador: Intel Core } \\
\text { i5 } 2.0 \text { 6Hz } \\
\text { Disco Duro: } 360 \text { 6B } \\
\text { Memoria RaM:4 6B } \\
\text { Tarjeta de Red: Gigahit } \\
\text { Ethemet } \\
\text { Sistema Operativo: } \\
\text { Windows Server } 2012\end{array}$ & $\begin{array}{l}\text { Servidor } \\
\text { Domain }\end{array}$ & No Aplica & 6КОСа2 & $\begin{array}{l}\text { Disco Duro: } 80 \text { 6B } \\
\text { Memoria RAM: } 1 \text { 6B } \\
\text { Sistema Operativo: } \\
\text { Windows Server }\end{array}$ & \\
\hline & & $\begin{array}{c}\text { Servidar de } \\
\text { Base de } \\
\text { Datos }\end{array}$ & No aplica & GKBDal & \begin{tabular}{|l|} 
Disco Duro: 28 6B \\
Memoria RAM: 2 6B \\
Sistema Operativo: \\
Windows Server
\end{tabular} & \\
\hline \multirow[t]{2}{*}{ GXSVor } & $\begin{array}{l}\text { Procesador: Intel Core } \\
\text { is } 2.0 \text { 6Hz } \\
\text { Disco Duro: } 360 \text { 6B } \\
\text { Memoria RAM: } 6 \text { 6B } \\
\text { Tarjeta de Red: Gigahit } \\
\text { Ethernet } \\
\text { Sistema Operativo: } \\
\text { Windows Server } 2012\end{array}$ & $\begin{array}{c}\text { Servidor de } \\
\text { Base de } \\
\text { Datos }\end{array}$ & No Aplica & GKBDo2 & $\begin{array}{l}\text { Disco Duro: } 23 \text { 6B } \\
\text { Memoria RAM: } 1 \text { 6B } \\
\text { Sistema Operativo: } \\
\text { LINuX }\end{array}$ & \\
\hline & & $\begin{array}{l}\text { Servidor de } \\
\text { Base de } \\
\text { Datos }\end{array}$ & No aplica & GKBD03 & \begin{tabular}{|l|} 
Disco Duro: 29 68 \\
Memoria RAM: 2 6B \\
Sistema Operativo: \\
Windows Server
\end{tabular} & \\
\hline
\end{tabular}




\begin{tabular}{|c|c|c|c|c|c|c|}
\hline & & $\begin{array}{c}\text { Servidor de } \\
\text { Base de } \\
\text { Datos }\end{array}$ & No aplica & 6KBDD4 & $\begin{array}{l}\text { Disco Duro: } 30 \text { 6B } \\
\text { Memoria RAM: } 2 \text { 6B } \\
\text { Sistema Operativo: } \\
\text { Windows Server }\end{array}$ & \\
\hline \multirow[t]{4}{*}{ 6xSWo3 } & $\begin{array}{l}\text { Procesador: Intel Core } \\
\text { is } 2.0 \text { GHz } \\
\text { Disco Duro: } 380 \text { 6B } \\
\text { Memoria RAM: } 6 \text { 6B } \\
\text { Tarjeta de Red: Gigabit } \\
\text { Ethemet } \\
\text { Sistema Operativo: } \\
\text { Windows Server } 2012\end{array}$ & Servidor web & No aplica & GKWEBOI & $\begin{array}{l}\text { Disco Duro: } 3668 \\
\text { Memoria RAM:1 68 } \\
\text { Sistema Operativo: } \\
\text { Windows Server }\end{array}$ & \\
\hline & & Servidor web & No aplica & GKWEE02 & $\begin{array}{l}\text { Disco Duro: } 80 \text { 6B } \\
\text { Memoria RAM: } 2 \text { 6B } \\
\text { Sistema Operativo: } \\
\text { Windows Server }\end{array}$ & \\
\hline & & Servidor web & No aplica & GKWEE03 & $\begin{array}{l}\text { Disco Duro: } 41 \text { GB } \\
\text { Memoria RAM: } 2 \text { GB } \\
\text { Sistema Operativo: } \\
\text { Windows Server }\end{array}$ & \\
\hline & & Servidor web & No aplica & GKWEED4 & $\begin{array}{l}\text { Disco Duro: } 41 \text { 6B } \\
\text { Memoria RAM: } 2 \text { 6B } \\
\text { Sistema Operativo: } \\
\text { Windows Server }\end{array}$ & \\
\hline GKWEBD5 & $\begin{array}{l}\text { Procesador: Intel Core } \\
\text { is } 2.06 \mathrm{GHz} \\
\text { Disco Duro: } 300 \text { 6B } \\
\text { Memoria RAM: } 2 \text { 6B } \\
\text { Tarjeta de Red: Gigahit } \\
\text { Ethernet } \\
\text { Sistema Operativo: } \\
\text { Windows }\end{array}$ & Servidor Web & $\begin{array}{l}\text { Contiene ol } \\
\text { servidor web }\end{array}$ & No aplica & No aplica & TI \\
\hline БКWEВ06 & $\begin{array}{l}\text { Procesador: Intel Core } \\
\text { is } 2.0 \mathrm{GHz} \\
\text { Disco Duro: } 300 \text { 6B } \\
\text { Memoria RAM:2 6B } \\
\text { Tarjeta de Red: Gigahit } \\
\text { Ethernet } \\
\text { Sistema Operativo: } \\
\text { Windows }\end{array}$ & Servidor Web & $\begin{array}{l}\text { Contiene ol } \\
\text { servidor web }\end{array}$ & No aplica & No aplica & Tा \\
\hline
\end{tabular}




\begin{tabular}{|c|c|c|c|c|c|c|}
\hline GKBIOI & \begin{tabular}{|l} 
Procesador: Intel Core \\
is 2.0 GHz \\
Disco Duro: 300 6B \\
Memoria RAM:2 6B \\
Tarjeta de Red: Gigabit \\
Ethernet \\
Sistema Operativo: \\
Windows
\end{tabular} & SQL BackLlps & No aplica & No aplica & No aplica & TI \\
\hline 6KBlo2 & $\begin{array}{l}\text { Procesador: Intel Core } \\
\text { is } 2.0 \text { GHz } \\
\text { Disco Duro: } 300 \text { 6B } \\
\text { Memoria RAM:2 6B } \\
\text { Tarjeta de Red: Gigahit } \\
\text { Ethernet } \\
\text { Sistema Operativo: } \\
\text { LINUX }\end{array}$ & $\begin{array}{c}\text { SQL } \\
\text { Prraducción }\end{array}$ & & No aplica & No aplica & ता \\
\hline 6KBlo3 & $\begin{array}{l}\text { Procesador: Intel Core } \\
\text { is } 2.0 \text { 6Hz } \\
\text { Disco Duro: } 300 \text { 6B } \\
\text { Memoria RAM: } 2 \text { 6B } \\
\text { Tarjeta de Red: Gigahit } \\
\text { Ethemet } \\
\text { Sistema Operativo: } \\
\text { LNuX }\end{array}$ & Servidor Web & & No aplica & No aplica & TI \\
\hline \multirow[t]{3}{*}{ GKSVO4 } & \begin{tabular}{|l} 
Procesador: Intel Core \\
is 2.0 GHz \\
Disco Duro: 380 6B \\
Memoria RAM:6 6B \\
Tarjeta de Red: Gigabit \\
Ethernet \\
Sistema Operativo: \\
Windows Server 2012 \\
\end{tabular} & Servidor web & No aplica & GKBID4 & $\begin{array}{l}\text { Disco Duro: } 30 \text { 68 } \\
\text { Memoria RAM: } 168 \\
\text { Sistema Operativo: } \\
\text { Windows Server }\end{array}$ & त \\
\hline & & Servidor Web & No aplica & GKBII05 & $\begin{array}{l}\text { Disco Duro:41 6B } \\
\text { Memoria RAM: } 2 \text { 6B } \\
\text { Sistema Operativo: } \\
\text { Windows Server }\end{array}$ & Tा \\
\hline & & SQL BackLlps & No aplica & GKBla6 & $\begin{array}{l}\text { Disco Duro: 20 6B } \\
\text { Memoria RaM:1 } 68 \\
\text { Sistema Operativo: } \\
\text { UINux }\end{array}$ & TI \\
\hline
\end{tabular}


2. Diagrama de Infraestructura:

21. Infraestructura fisica:-

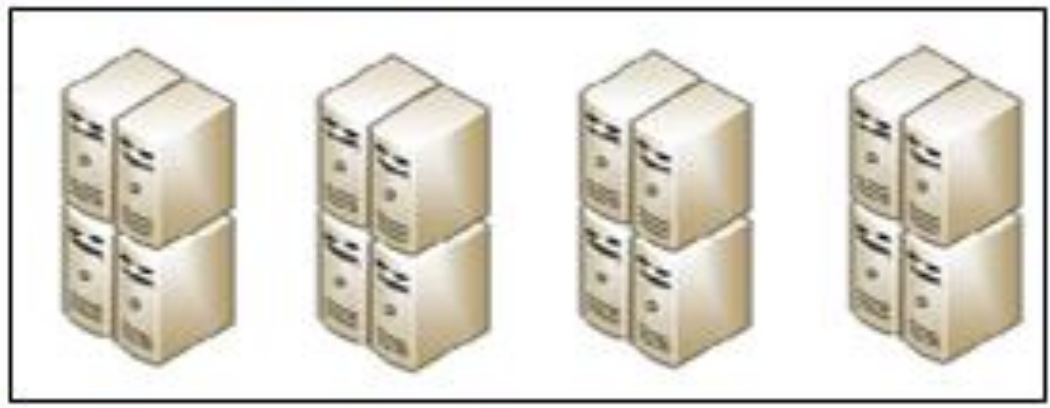

22. Infiestructura con Software Base
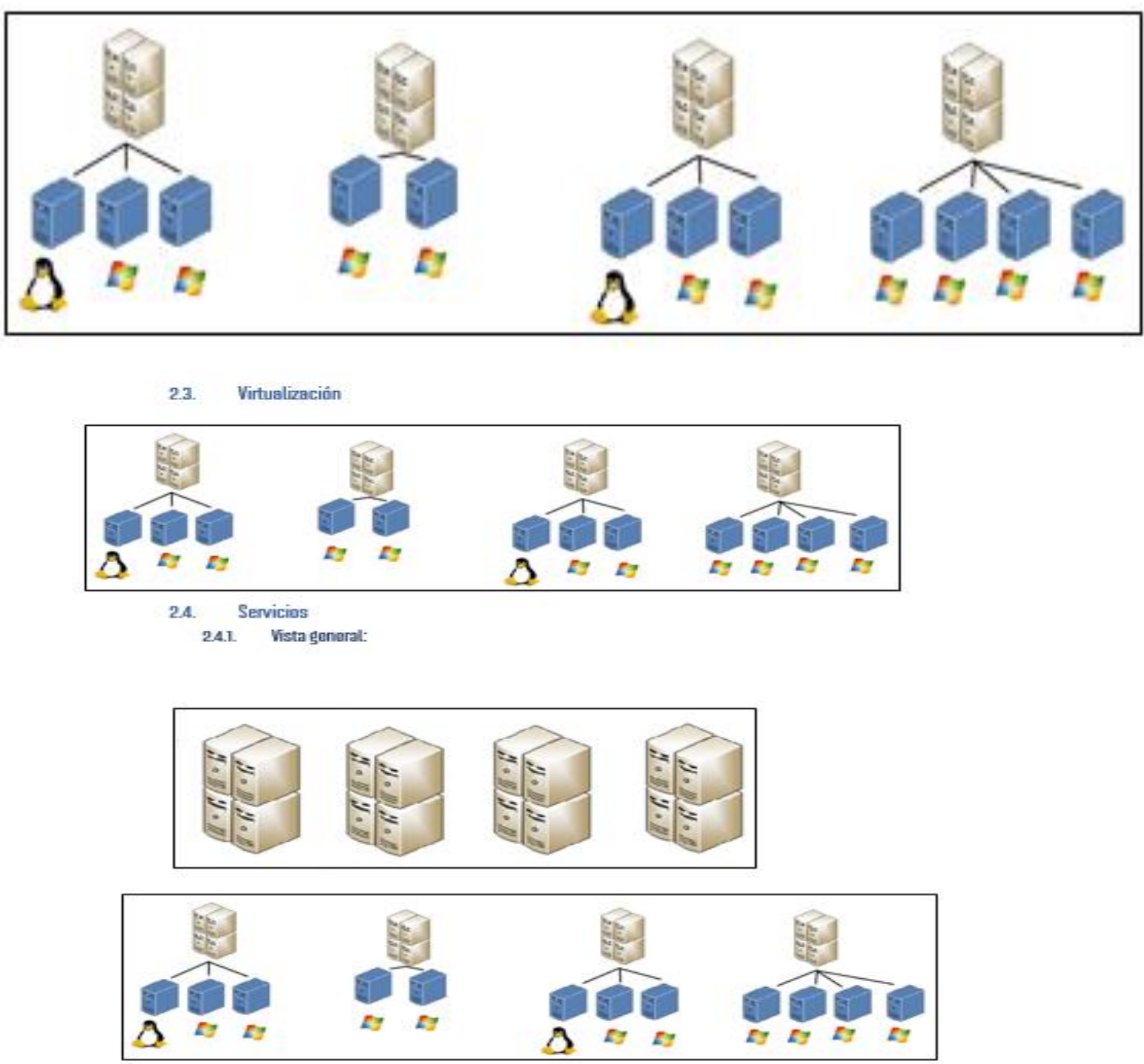


\section{ANEXO 9: ESPECIFICACIÓN DE REQUERIMIENTOS}

\section{Datos Generales del Proyecto}

Nombre del Proyecto:

Modelo de Miǵración a la nube de los servidores de un Data Center

\begin{tabular}{|c|c|}
\hline Lider lsuario & Especialista Cloud \\
\hline Jimmy Novoa & Christian Rojas \\
\hline Fecha de hnicio & Fecha de Aprobacioin \\
\hline $23 / 08 / 2017$ & $25 / 08 / 2017$ \\
\hline
\end{tabular}

\section{Antecedentes}

Globokas Perú es subsidiaria de GTV GlobokasNet LLC. curya sede está en Maryland EE.UU. Fue fundada en el Perú en el año २००? para extender la presencia y cobertura de las entidades financieras y comerciales a nivel nacional.

\section{Ohjetivo del Requerimiento}

- Capacidad de escalabilidad de acuerdo al crecimiento la organización.

- Reducir gastos operativos (Mantenimiento de servidores, servicio de luz, sistemas de refrigeraciòn, entre otros.)

3. Descripciín del Requerimients

3.l. Alcance Funcional

Migrar a una nube pública sus servidores.

3.2. Requerimientos Funcionales

Se requiere poder realizar solcitudes a los servidores en cloud desde algún cliente y que estos las atiendan correctamente.

3.3. Requerimientos No Funcionales

Se requiere que la plataforma en la que se vaya a utilizar, sea seǵura, eficiente y usable

\subsection{Exclusiones (a ser llenado por el Especialista)}

Se miǵrarán los servidores de acuerdo al resultado de una evalución de carǵas de trabajo y la disponibildiad de crédito de la plataforma Coud.

4. Alcance Técnico

-Las miǵraciones se realizarán por medio de una Command line Interface (CL), haciendo uso del API correspondiente de la plataforma a utilizar.

\section{Consideraciones}

- Cualquier cambio en el alcance descrito durante el desarrollo será evaluado como un control de cambio o una mejora adicional que deberá aprobarse por el usuario previamente para ser planificada. 


\section{ANEXO 10: BENCHMARKING}

\section{Alcance}

En el presente documento se presentará una comparación de las dfferentes plataformas de la tecnoloǵia de Cloud Computing en el mercado ǵlobat lo cual nos ayudará a realizar la elección más viable a través de dfferentes criterios de calidad, obteniendo camo resultado final la utilización de la mejor plataforma dentro del proyecto.

\section{Descripción del Activo Preexistente}

Uno de las abjetivos del proyecto se basa en la miǵración a la nube de los servidores de la empresa Data Center. Para ello se ha considerado el inventario de hardware que actualmente función en la emprese:

\section{Alternativas a considerar}

Para la elección de las alternativas a tener en consideración dentro del presente documento, se ha tenida como foco principal el posicionamiento de la herramienta dentro del mercado como casos de éxito, soluciones tap y herramientas que cubran la mayoria de necesidades de las empresas de hoy en día como la implementación de alşuna Cloud pública.

Para ello se ha escoǵido las plataformas más conocidas en elmercado: Amazon Web Services (AWS), Gooģle Cloud Plafforco IBM Bluemixy Microsoft Azure.

Para el caso de Amazon Web Services, quien se considera pionero en este campo. Alquila sevvidores para alojar web sites, puede acabar rebajando sus precios. Cluster GPU, webservices" por ejemplo, permite realizar operaciones que muestren los complejos śráficos y altas velocidades.

Por atro lado, Gooǵle Cloud Platforon es un conjunto de servicios de cloud camputiné públicos ofrecidos por Booǵle. la plataforma incluye una gaama de servicios alojados para el cálculo, almacenamiento y desarrollo de aplicaciones que se ejecutan en el hardware de Google. Los desarrolladores de software, los administradores de la nubey otros profesionales de II de la empresa pueden acceder a los sevvicios de Gongle Cloud Platforma Google Cloud Platform través de la Internet pública a través de una conexión de red dedicada.

Hablenda de empresas de ǵran trayectoria, IBM posee Bluemix, el cual es una plataforma de nube como servicio (Bag,5) desarrollado por IBM. Saporta varios lenǵgujes de prógramación y servicios, asi como Dexllos inteśrado para construir, ejecutar, implementar y administrar aplicaciones en la nube. Bluemix se basa en la tecnoloşia abierta Cloud Foundry y se ejecuta en la infreestructura Softlazyec, Bluemix soporta varios lenéuajes de próśramación incluyendo Java, Node.js, Go, PHP, Swift, Python, Ruby Sinatra, Ruby an Rails y puede extenderse para soportar otros lenguajes como \$calla mediante el uso de buldpacks.

Finalizando con las alternativas, se tiene Azure, este es una plataforma de nube abierta y flexible que permite compilar, implementar y administrar aplicaciones rápidamente en una red ǵlabal de centros de datos administrados por Microsoft. Puede compilar aplicaciones en cualquier lenģuaje, herramienta o marco, permitiendo además intȩ́rar sus aplicaciones de nube públicas con el entorno de TI
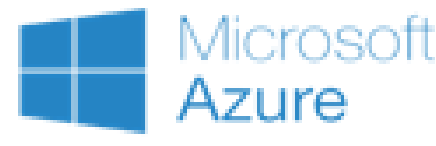
existente. 


\section{Análisis Comparativo}

a) Propósito de evaluación

Comparar les caracteristicas de las diferentes plataformas identificados como disponibles en el mercado ślobal, seleccionando aquel que cumpla con las necesidades del proyecto satisfactoriamente.

b) Madela de calidad

a. Para establecer el Madelo de Calidad a utilizar dentro de la evaluación se consideró la ISL/IEC 25000, conocida como SQuaRE(System and Software Quality Requirements and Evaluation), que tiene por abjetivo la creación de un marco de trabajo común para evaluar la calidad del producto software.

b. En base al modelo de calidad para la calidad externa e interna se establecieron los atributos a valurar para el análisis de la herramienta a escoǵer.

- Funcionalidad

- Fiabilidad

- Usabilidad

- Eficiencia

- Mantenimiento

- Portabilidad

- Eficacia

- Productividad

- Satisfacción

- Sesuridad

c) Métricas

\begin{tabular}{|c|c|c|}
\hline Griterios de calficación & Escala de puntuaciain & Puntaraciin \\
\hline Cumple totalmente & Avanzado & 80 a 100 \\
\hline Lumple parcialmente & Intermedio & $40 \div 80$ \\
\hline Cumple minimo & Básico & $20 \div 40$ \\
\hline
\end{tabular}

Puntaje minimo de aceptación

El puntaje minimo de aceptación de una alternativa será el de mayor a 80 puntos.

d) Evaluación técnica

Cuadro l: Evaluación técnica

\begin{tabular}{|c|c|c|c|c|}
\hline & Atributos & \multicolumn{2}{|c|}{ Descripción } & Puntaje \\
\hline 1 & \multicolumn{3}{|c|}{ Calidad interna y Externa } & \\
\hline 1.1 & Funcionalidad & \multicolumn{2}{|c|}{$\begin{array}{l}\text { Lumplimiento de las funciones principales al 100\% de } \\
\text { administrar servidores, memoria y redes, y } \\
\text { compatibilidad con sistemas operativos, etc. }\end{array}$} & 15 \\
\hline 1.2 & Fiabilidad & \multicolumn{2}{|c|}{$\begin{array}{l}\text { Capacidad para mantener un nivel especificado de } \\
\text { análisis y solución, en caso de errores. }\end{array}$} & 9 \\
\hline 1.3 & Usabilidad & \multicolumn{2}{|c|}{$\begin{array}{l}\text { Facilidad, rapidez de comprensión a nivel usuario y } \\
\text { técnico. }\end{array}$} & 10 \\
\hline \multirow[t]{2}{*}{1.4} & \multirow[t]{2}{*}{ Eficiencia } & Comportamiento & $\begin{array}{l}\text { Alto rendimiento para } \\
\text { análisis y procesos de } \\
\text { datos. }\end{array}$ & 6 \\
\hline & & Utilización de recursos & $\begin{array}{l}\text { El software debe } \\
\text { consumir recursus }\end{array}$ & 4 \\
\hline
\end{tabular}




\begin{tabular}{|c|c|c|c|c|}
\hline & & & $\begin{array}{l}\text { minimos de la PC, sin } \\
\text { afectar } \\
\text { multitarea } \\
\text { procesadoridad vs memoria. }\end{array}$ & \\
\hline \multirow[t]{2}{*}{1.5} & \multirow[t]{2}{*}{ Mantenimiento } & Actualización & $\begin{array}{l}\text { Facilidad para actualizar } \\
\text { de manera manual o } \\
\text { automática. }\end{array}$ & 5 \\
\hline & & Soporte & Servicio técnico local. & 5 \\
\hline \multirow[t]{2}{*}{1.6} & \multirow[t]{2}{*}{ Portabilidad } & Adaptabilidad & $\begin{array}{l}\text { Adaptable a distintos } \\
\text { entornos de software. }\end{array}$ & 7 \\
\hline & & Facilidad de instalación & $\begin{array}{l}\text { Contar con asistente de } \\
\text { instalación. }\end{array}$ & 9 \\
\hline 2 & \multicolumn{3}{|c|}{ Calidad de Uso } & \\
\hline 2.1 & Eficacia & \multicolumn{2}{|c|}{$\begin{array}{l}\text { Capacidad de alcanzar las metas operativas al } 100 \% \text { al } \\
\text { momento del uso de las funciones de administración de } \\
\text { servidores, memnria y redes por el usuario. }\end{array}$} & 9 \\
\hline 2.2 & Seǵridad & \multicolumn{2}{|c|}{$\begin{array}{l}\text { Capacidad de ser confiabley seéuro para la información } \\
\text { que se administre con dicha herramienta. }\end{array}$} & 7 \\
\hline 2.3 & Satisfacción & \multicolumn{2}{|c|}{$\begin{array}{l}\text { Capacidad para cubrir sus requerimientos con total } \\
\text { conformidad del usuario. }\end{array}$} & 7 \\
\hline 24 & Productividad & \multicolumn{2}{|c|}{$\begin{array}{l}\text { No deberá de consumir muchos recursos de memoria y } \\
\text { procesador en los equipos donde se hayan instalado. }\end{array}$} & 7 \\
\hline
\end{tabular}

Cuadro Comparativo № I: Análisis Técnico

\begin{tabular}{|c|c|c|c|c|c|c|c|}
\hline \multirow[b]{3}{*}{1} & \multirow{2}{*}{\multicolumn{2}{|c|}{ Atributes }} & \multirow{2}{*}{ Puntipi } & \multicolumn{4}{|c|}{ Mtomatives } \\
\hline & & & & \multicolumn{4}{|c|}{ Prtefunins Dind } \\
\hline & \multicolumn{2}{|c|}{ CALIDAD DXTERNA EIKTERMA } & 70 & $\begin{array}{l}\text { Amazon Web } \\
\text { Services }\end{array}$ & $\begin{array}{c}\text { Google Cloud } \\
\text { Platform }\end{array}$ & IBM BLUEMIX & Microsoft Azure \\
\hline 1.1 & \multicolumn{2}{|c|}{ Funcionalidad } & 15 & 14 & 10 & 12 & 15 \\
\hline 1.2 & \multicolumn{2}{|c|}{ Fiabilidad } & 9 & 9 & 9 & 9 & 9 \\
\hline 1.3 & \multicolumn{2}{|c|}{ Usabilidad } & 10 & 10 & 10 & 10 & 10 \\
\hline \multirow[b]{2}{*}{1.4} & \multirow[b]{2}{*}{ Eficiencia } & Comportamiento & 6 & 6 & 6 & 6 & 6 \\
\hline & & $\begin{array}{l}\text { Utilización de } \\
\text { recursos }\end{array}$ & 4 & 4 & 4 & 4 & 4 \\
\hline \multirow{2}{*}{1.5} & \multirow{2}{*}{ Mantenimiento } & Actualización & 5 & 5 & 5 & 5 & 5 \\
\hline & & Soporte & 5 & 5 & 5 & 5 & 5 \\
\hline \multirow[b]{2}{*}{1.6} & \multirow[b]{2}{*}{ Portabilidad } & Adaptahidad & 7 & 7 & 7 & 7 & 7 \\
\hline & & $\begin{array}{l}\text { Facilidad de } \\
\text { instalación }\end{array}$ & 9 & 9 & 9 & 9 & 9 \\
\hline \multicolumn{3}{|c|}{ Sub Total } & 70 & 69 & 65 & 67 & 70 \\
\hline 2 & \multicolumn{2}{|c|}{ CALIDAD DE USD } & 30 & $\begin{array}{c}\text { Amazon Web } \\
\text { Services }\end{array}$ & $\begin{array}{c}\text { Google Cloud } \\
\text { Platform }\end{array}$ & IBM BLUEMIX & Microsoft Azure \\
\hline 2.1 & \multicolumn{2}{|c|}{ Eficacia } & 9 & 8 & 8 & 7 & 8 \\
\hline 2.2 & \multicolumn{2}{|c|}{ Seguridad } & 7 & 7 & 5 & 7 & 7 \\
\hline 2.3 & \multicolumn{2}{|c|}{ Satisfacción } & 7 & 7 & 6 & 7 & 7 \\
\hline 2.4 & \multicolumn{2}{|c|}{ Productividad } & 7 & 7 & 7 & 7 & 7 \\
\hline \multicolumn{3}{|c|}{ Sub Total } & 30 & 29 & 26 & 28 & 29 \\
\hline
\end{tabular}


Cuadro Comparativo № 2: Análisis Costo Beneficio

\begin{tabular}{|c|c|c|c|c|c|}
\hline \multirow{2}{*}{$\nabla \cdot$} & \multirow{2}{*}{ Criturinse Evalus } & \multicolumn{4}{|c|}{ Wuba Publin } \\
\hline & & Amerenn Whb Services & Eongle Lnod Catfom & IBN Blurmix & Micmentt lane \\
\hline & & \$L108 / Hora - & $\$ 0035 /$ Hora - & \$0.105 / Hora - & \$025/Hora \\
\hline 1 & $\begin{array}{l}\text { Costo de Servicio / } \\
\text { licencia }\end{array}$ & \$52.56/Nensualpor 1 Añ̄o & $\$ 25.55 /$ Mensual & $\$ 70.00 /$ Mensual & $\$ 1860 /$ Mensual \\
\hline 2 & Caracteristicas & $\begin{array}{l}1 \text { Cloud Server: } 1.0 \text { fobz Corey } \\
4 \text { GB RAY }\end{array}$ & $\begin{array}{l}\text { 1Cloud Server:10 fobz } \\
\text { Core y } 4 \text { GB RAM }\end{array}$ & $\begin{array}{l}1 \text { Cloud Server: } 2.0 \text { fobz } \\
\text { Core y } 4 \text { GB RAM }\end{array}$ & $\begin{array}{l}1 \text { Cloud Server. } 20 \text { fotz } \\
\text { Core y } 2 \text { GB RAM }\end{array}$ \\
\hline 3 & $\begin{array}{c}\text { Soportey } \\
\text { manterimiento }\end{array}$ & 24 horas, 7 dias a la semena & $\begin{array}{c}24 \text { hores, } 7 \text { dlas a la } \\
\text { semana }\end{array}$ & $\begin{array}{c}24 \text { hores, } 7 \text { dlas a la } \\
\text { semana }\end{array}$ & $\begin{array}{c}24 \text { hores, } 7 \text { dlas a la } \\
\text { semana }\end{array}$ \\
\hline
\end{tabular}

Cuadro N09: Resumen Global

\begin{tabular}{|c|c|c|c|c|}
\hline \multirow{2}{*}{ Anflisis } & \multicolumn{4}{|c|}{ Nuba Pitlix } \\
\hline & Anrasen Whb Services & Gougla & IBM Softilawn & Micrneuft lane \\
\hline $\begin{array}{l}\text { Anélisis Comparativo } \\
\text { Técnico }\end{array}$ & 97 & gl & 95 & 96 \\
\hline & sulus / Hora - & \$an35/Hora- & \$n105/Hora - & $\$ 0.25 /$ Hora \\
\hline Anélisis Costo Beneficio & \$52.55/Mensual par 1 Añ̃ & $\$ 25.55 /$ Nensual & $\$ 70$ nal/Mensual & \$1860/Nensual \\
\hline
\end{tabular}

\section{Conclusiones}

Tras el análisis realizado a las alternativas de plataforma a utilizar en el proyecto se concluyó lo siéuiente:

- Una vez finalizado la asiǵnación de puntajes de acuerdo a los criterios definidos, se suma el puntaje total de cada plataforma identificado.

- La herramienta que contenǵga la mayor puntuación será la utilizada dentro de la arquitectura tecnológica a implementar.

- El servicio escogido para la plataforma y que será recumendado en el proyecto en relación caracteristicas técnicas es Amazon Web Services 


\section{ANEXO 11: ESTUDIO DE VIABILIDAD}

\section{ESTUDIO DE VIABILIDAD TECNICA}

\section{a. DESARROLLO}

\begin{tabular}{|c|c|c|c|c|c|c|}
\hline Numero & Servidor a Migar & Caracteristicas & Valor & Dificultad & $\begin{array}{l}\text { Dif icultad } \\
\text { Acumulada }\end{array}$ & Resultado \\
\hline \multirow{5}{*}{1} & \multirow{5}{*}{ GNDCO1 } & Sisterna Operativo & Linux & 2 & \multirow[t]{5}{*}{ 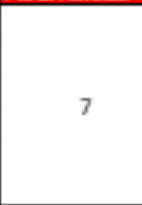 } & \multirow{5}{*}{ Mgración Compleja Factible } \\
\hline & & Disco Duro Oajpodo(GB) & 20 & 1 & & \\
\hline & & Formato Disco Duro & ONF & 2 & & \\
\hline & & ServidorVirtualizado & No & 2 & & \\
\hline & & Contiene cargas de trabajo relacion ado aregul ación sensi ble & No & 0 & & \\
\hline \multirow{5}{*}{2} & \multirow{5}{*}{ GKDCO2 } & Sisterna Operativo & Windaws & 1 & \multirow{5}{*}{21} & \multirow{5}{*}{ Mgración no Factible } \\
\hline & & Disco Duro Ocupodo $(G B]$ & 80 & 3 & & \\
\hline & & Formato Disca Duro & ONF & 2 & & \\
\hline & & Servidar Virtualizado & 5 & 0 & & \\
\hline & & Contiene cargas de trabajo relacion ado a regul acián sensi ble & Si & 15 & & \\
\hline \multirow{5}{*}{3} & \multirow{5}{*}{ GKBD01 } & Sisterna Operativo & Windaws & 1 & \multirow{5}{*}{5} & \multirow{5}{*}{ Mgracián Sencilla } \\
\hline & & Disco Duro Oaupodo[GB] & 28 & 2 & & \\
\hline & & Famato Disca Duro & OVF & 2 & & \\
\hline & & ServidarVirtualizado & Si & 0 & & \\
\hline & & Contiene cargas de trabajo relacion ado a regul ación sensi ble & No & 0 & & \\
\hline \multirow{5}{*}{4} & \multirow{5}{*}{ GKBDO2 } & Sisterna Operativo & Linux & 2 & \multirow{5}{*}{6} & \multirow{5}{*}{ Migración Compleja Factible } \\
\hline & & Disco Dura Oaspodo(GB] & 23 & 1 & & \\
\hline & & Fomato Disca Duro & VDI & 3 & & \\
\hline & & Servidor Virtualizado & Si & 0 & & \\
\hline & & Contiene cargas de trabajo relacion ado aregul ación sensi ble & No & 0 & & \\
\hline \multirow{5}{*}{5} & \multirow{5}{*}{ GKBDO3 } & Sisterna Operativo & Windows & 1 & \multirow{5}{*}{5} & \multirow{5}{*}{ Mgración Sencilla } \\
\hline & & Disco Duro Oaupodo[GB] & 29 & 2 & & \\
\hline & & Fonmato Disca Duro & OVF & 2 & & \\
\hline & & Servidor Virtualizado & Si & 0 & & \\
\hline & & Contiene cargas de trabajo relacion ado aregul ación sensi ble & No & 0 & & \\
\hline
\end{tabular}

\begin{tabular}{|c|c|c|c|c|c|c|}
\hline \multirow{5}{*}{6} & \multirow{5}{*}{ GKBDO4 } & Sistema Operativo & Windows & 1 & \multirow{5}{*}{ s } & \multirow{5}{*}{ Mgradión Sendila } \\
\hline & & Dlsco Duro Daupdo(GB) & 30 & 2 & & \\
\hline & & Fomato Disco Duro & OVF & 2 & & \\
\hline & & Servidor Virtuslilisdo & Sil & 0 & & \\
\hline & & Conticne cargas de trabajo relocionsdo aregul adion sensl bic & No & 0 & & \\
\hline \multirow{5}{*}{7} & \multirow{5}{*}{ GKWEBOI } & Sistema Operativo & Windows & 1 & \multirow{5}{*}{5} & \multirow{5}{*}{ Mgradion Sendils } \\
\hline & & Disco Duro Daupsdo(GB) & 36 & 2 & & \\
\hline & & Fomato Disco Duro & OVF & 2 & & \\
\hline & & Servidor Vi itualilisdo & Sil & 0 & & \\
\hline & & Contiene cargas de trabajo relocionsdo aregul sdón sensi bic & No & o & & \\
\hline \multirow{5}{*}{8} & \multirow{5}{*}{ GKWEBC2 } & Sistema Operativo & Windows & 1 & \multirow{5}{*}{6} & \multirow{5}{*}{ Mgradon Compleja Factibil } \\
\hline & & Disco Duro Daupsdo(GB) & 80 & 3 & & \\
\hline & & Formato Dlsco Duro & QVF & 2 & & \\
\hline & & ServidorVirtusallisdo & S1 & 0 & & \\
\hline & & Contiene cargas de trabajo relacionsdo aregul adion sensi bic & No & o & & \\
\hline \multirow{5}{*}{9} & \multirow{5}{*}{ GKWEBC3 } & Sistema Operativo & Windows & 1 & \multirow{5}{*}{5} & \multirow{5}{*}{ Mgradión Sendila } \\
\hline & & Disco Duro Daupsdo(GB) & 41 & 2 & & \\
\hline & & Fomato Dlaco Duro & ONF & 2 & & \\
\hline & & ServidorVirtusallizado & Sil & 0 & & \\
\hline & & Conticne cargas de trabajo re locion ado aregul adón sensi bic & No & 0 & & \\
\hline \multirow{5}{*}{10} & \multirow{5}{*}{ GKWEBCA } & Slstema Operativo & Windows & 1 & \multirow{5}{*}{ s } & \multirow{5}{*}{ Mgradión Sendila } \\
\hline & & Disco Duro Daupsdo(GB) & 41 & 2 & & \\
\hline & & Fomato Dlisco Duro & ONF & 2 & & \\
\hline & & ServidorVirtuslilisdo & 5l & 0 & & \\
\hline & & Conticne cargas de trabajo relocionsdo aregul adón sensi bic & No & 0 & & \\
\hline
\end{tabular}




\begin{tabular}{|c|c|c|c|c|c|c|}
\hline \multirow{5}{*}{11} & \multirow{5}{*}{ GKWEBOS } & Sistema Operativo & Windows & 1 & \multirow{5}{*}{7} & \multirow{5}{*}{ Migración Compleja Facti bic: } \\
\hline & & Disco Duro Oaupado(GB) & 쪼 & 2 & & \\
\hline & & Formato Disco Duro & OVF & 2 & & \\
\hline & & Servidor Virtualizado & No & 2 & & \\
\hline & & Conticne cargar de trabajo relaci onado a regul ación sensible & No & o & & \\
\hline \multirow{5}{*}{12} & \multirow{5}{*}{ GKWEBOG } & Sistema Operativo & Mindows & 1 & \multirow{5}{*}{8} & \multirow{5}{*}{ Migración Compleja Factible } \\
\hline & & Disco Duro Oaup ado(GB) & 58 & 3 & & \\
\hline & & Formato Disco Duro & OVF & 2 & & \\
\hline & & Servidor Virtual Izado & No & 2 & & \\
\hline & & Contiene cargas de trabajo relaci onado a regul ación sensible & No & 0 & & \\
\hline \multirow{5}{*}{13} & \multirow{5}{*}{ GKBIOO1 } & Sistema Operativo & Windows & 1 & \multirow{5}{*}{8} & \multirow{5}{*}{ Migración Compleja Factibie } \\
\hline & & Disco Duro Oaup ado(GB) & $\mathrm{Bg}$ & 3 & & \\
\hline & & Formato Disco Duro & OVF & 2 & & \\
\hline & & Servidor Virtualizado & No & 2 & & \\
\hline & & Contiene cargas de trabajo relacionado a regul ación sensible & No & o & & \\
\hline \multirow{5}{*}{14} & \multirow{5}{*}{ GKBIOCR } & $\begin{array}{r}\text { Sistema Operativo } \\
\end{array}$ & Linux & 2 & \multirow{5}{*}{9} & \multirow{5}{*}{ Migración Muy Compleja } \\
\hline & & Disco Duro Oaup ado(GB) & 56 & 3 & & \\
\hline & & Formato Disco Duro & OVF & 2 & & \\
\hline & & Servidor Virtual Izado & No & 2 & & \\
\hline & & Contiene cargas de trabajo relacionado a regul ación sensible & No & 0 & & \\
\hline \multirow{5}{*}{15} & \multirow{5}{*}{ GKBIOCB } & Sistema Operativo & Linux & 2 & \multirow{5}{*}{9} & \multirow{5}{*}{ Migración Muy Compleja } \\
\hline & & Disco Duro Oaup ado(GB) & $\pi 2$ & 3 & & \\
\hline & & Formato Dlsco Duro & OVF & 2 & & \\
\hline & & Servidor Virtualizado & No & 2 & & \\
\hline & & Conti ene cargas de trabajo rel aclonado a regul ación sensible & No & 0 & & \\
\hline \multirow{5}{*}{16} & \multirow{5}{*}{ GKBIOO4 } & Sistema Operativo & Windows & 1 & \multirow{5}{*}{5} & \multirow{5}{*}{ Mgración Sencilla } \\
\hline & & Dlsco Duro Oaup ado(GB) & 20 & 1 & & \\
\hline & & Formato Disco Duro & $\mathrm{VDI}$ & 3 & & \\
\hline & & Servidor Virtual Izado & si & 0 & & \\
\hline & & Conticne cargas de trabajo rel acionado a regul ación sensible & No & 0 & & \\
\hline
\end{tabular}

\begin{tabular}{|c|c|c|c|c|c|c|}
\hline \multirow{5}{*}{17} & \multirow{5}{*}{ GKBIO05 } & Sistema Operativo & Windows & 1 & \multirow{5}{*}{19} & \multirow{5}{*}{ Mgración no Factible } \\
\hline & & Disco Duro Ocupado(GB) & 41 & 2 & & \\
\hline & & Formato Disco Duro & VHD & 1 & & \\
\hline & & Servidor Virtual izado & Si & 0 & & \\
\hline & & Contiene cargas de trabajo rel acion ado a regul ación sensible & Si & 15 & & \\
\hline \multirow{5}{*}{18} & \multirow{5}{*}{ GKBIOO6 } & Sistema Operativo & Linux & 2 & \multirow{5}{*}{5} & \multirow{5}{*}{ Migración Senailla } \\
\hline & & Disco Duro Ocupado(GB) & 20 & 1 & & \\
\hline & & Format o Disco Duro & OVF & 2 & & \\
\hline & & Servidor Vir tual izado & $\mathrm{Si}$ & 0 & & \\
\hline & & Contiene cargas de trabajo rel acion ado a regul ación sensible & No & 0 & & \\
\hline
\end{tabular}

\section{b. CONCLUSION}

Se lleģó a la conclusión que existen 2 servidores no factibles a miǵrar, el resto de servidores (físicos y

virtuales) son aptos para la miéración. 


\section{ESTUDIO DE VIABILIDAD ECONOMICA}

a. DESARROLLO

i. COSTOS EN TECNOLogIa

COSTOS DE SOFWARE HARDWARE Y PERSONAL

\begin{tabular}{|c|c|c|c|c|c|c|}
\hline & & & & & Tipo de Cambio & $5 / 3.30$ \\
\hline \multicolumn{7}{|l|}{ Gastos } \\
\hline & Cantidad & Pracio Unitario(\$) & Horas/Mes & Prgis Iotal(S/.) & Murmero de Priodos & Costs Iotal \\
\hline \multicolumn{7}{|l|}{ 1.Implementacion de la Herramienta } \\
\hline \multicolumn{7}{|l|}{ A dquicision de Infrae structura } \\
\hline PCs Usua rio & & Existe & & 0 & & \\
\hline SubTotal & & 0 & & 0 & & \\
\hline \multicolumn{7}{|l|}{ Plataforma Cloud } \\
\hline Servidor en cloud (insta ncia s)VM CLOU & 18 & $\$ 16.56$ & 720.00 & $\mathrm{~S} / 983.66$ & 12 & 11803.97 \\
\hline \begin{tabular}{|l|l|} 
Elastic Block Store \\
\end{tabular} & 18 & $\$ 5.00$ & 720.00 & $\mathrm{~S} / 297.00$ & 12 & 3564.00 \\
\hline SubTotal & & $\$ 21.56$ & & $\mathrm{~S} / 1,280.66$ & & \\
\hline Subtotal infrae structura y cloud & & & & $\mathrm{S} / 1,280.66$ & & 15367.97 \\
\hline \multicolumn{7}{|l|}{ 2. Costos de Personal } \\
\hline Especialista Cloud & 1 & $\$ 2,080.00$ & 160.00 & $\mathrm{~S} / 6,864.00$ & 2 & $\mathrm{~S} / 13,728.00$ \\
\hline Jefe de Proyecto & 1 & $\$ 2,400.00$ & 160.00 & $\mathrm{~S} / 7,920.00$ & 2 & $S / 15,840.00$ \\
\hline A na lista de TI & 1 & $\$ 1,520.00$ & 160.00 & $\mathrm{~S} / 5,016.00$ & 2 & $\mathrm{~S} / 10,032.00$ \\
\hline Documenta dor & 2 & $\$ 100.00$ & 20.00 & $\mathrm{~S} / 660.00$ & 1 & $S / 660.00$ \\
\hline Personal de migración & 3 & $\$ 280.00$ & 40.00 & $\mathrm{~S} / 2,772.00$ & 1 & $S / 2,772.00$ \\
\hline SubT otal & & & & $\mathrm{S} / 23,232.00$ & & $\mathrm{~S} / 43,032.00$ \\
\hline 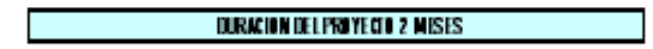 & & Costo & & S/. 58,399.97 & & \\
\hline
\end{tabular}

\section{ii. GRAFICO}

\section{Retorno de Inversión Globokast}

S/. $50,000.00$

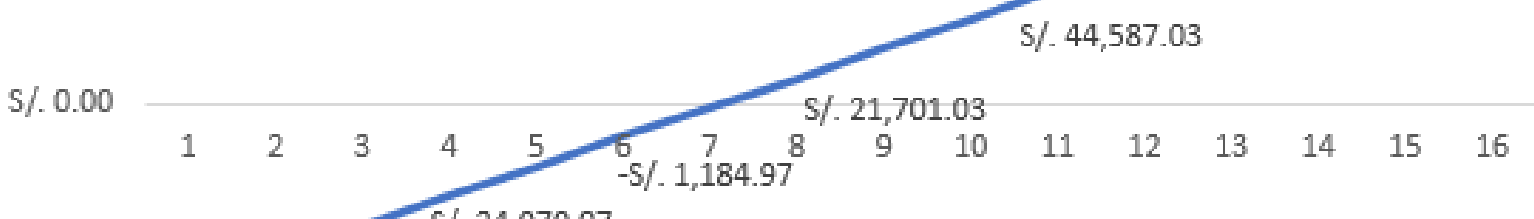

需/. 50,000.00

$-S / .24,070.97$

\section{$-S / .46,956.97$}

$100,000.00$

$-S / .150,000.00$

$-S / .200,000.00$

Mes 


\section{b. BENEFICIOS}

\section{CUADRO DE BENEFICIOS PARA UN CLIENIE QUUE MIGRA SUS SERVIIORES A CLOUID}

\begin{tabular}{|c|c|c|c|c|}
\hline GASTOS DE MANIENIMIENTO & MEIRICA & COSTOUNTARIO & COSTO MENSUAL & COSTO ANUAL \\
\hline Electricidad de siistema de refrigergción & 15 Kwh & $2,000.00$ & $2,000.00$ & S. $24,000.00$ \\
\hline Electricidad de los senvidores & 18 senvidores & 13.50 & 243.00 & $2,916.00$ \\
\hline Mantenimiento de senvidores & 18 senvidores & 500.00 & $9,000.00$ & S. $108,000.00$ \\
\hline Reposicion de inf & 1 Rep & 200.00 & 200.00 & S. $\quad 2,400.00$ \\
\hline \multirow[t]{2}{*}{ COSTOSDE PERSOMIL } & MIRICA & COSO UKIARIO & COSO MEKSJAL & COSTO AKJAL \\
\hline & & & $\mathrm{S} /$. & SI. \\
\hline & & Total & S/. $11,443.00$ & SI. $137,316.00$ \\
\hline
\end{tabular}

\section{c. CONCLUSION}

En conclusión, el proyecto es viable. Debido que lo invertido en la implementación se puede recuperar en aproximadamente 5 meses. Y seǵuir teniendo los beneficios cuantitativos.

\section{ESTUDIO DE VIABILIDAD OPERATIVA}

\section{a. CONCLUSION}

A los usuarios quienes va dirigido el modelo, se tiene conocimiento del manejo de los data centers (on premise) y estos seguirán con el monitoreo de estos, mediante las herramientas que brindan las plataformas en la nube. En caso de necesitar una capacitación, el proveedor de la plataforma brinda dichos servicios. Los encargados del área de Tl, desde el inicio han sido entusiastas con la implementación del modelo, puesto que tienen claro que esto les favorecerá y facilitara la tarea que a menudo realizan, por lo que existe el deseo de los usuarios directos de colaborar y participar en el proyecto

\section{CONCLUSION DE LA VIABILIDAD}

Demostrado en los puntos anteriores, tanto los estudios de factibilidad técnica, económica y operativa resultan ser favorables para el proyecto lo que determina su viabilidad. El proyecto resulta ser una buena implementación para la empresa, además a un costo no mayor que puede ser recuperado a corto plazo. 


\section{ANEXO 12: DOCUMENTO DE IMPLEMENTACIÓN}

\section{PROPÓSITO DEL DOCUMENTO}

El propósito del documento es detallar el paso a paso de la implementación en la plataforma cloud escogida (AWS).

\section{PROCESO DE MIGRACIÓN}

Para el presente documento se está considerando que ya se tenía instalado EC2-API-Tools, por lo tanto, se omitirá esta instalación.

- Primero se deben colocarlos tanto la clave pública y secreta de la cuenta de aws, la región y el formato de salida utilizando el comando aws configure en el CMD:

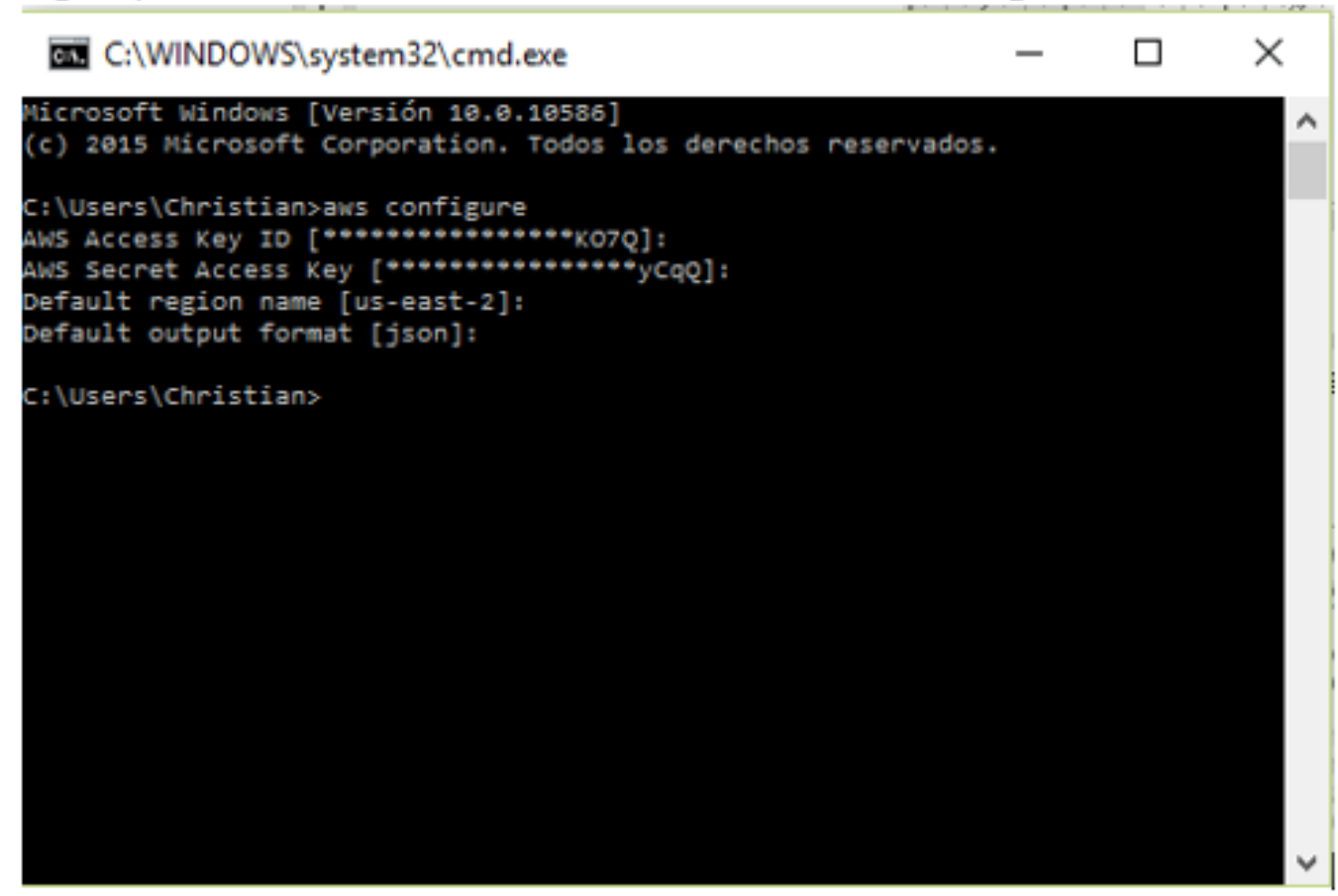

- Se creó el archivo trust-policy.json que permitirá crear el rol vmimport 


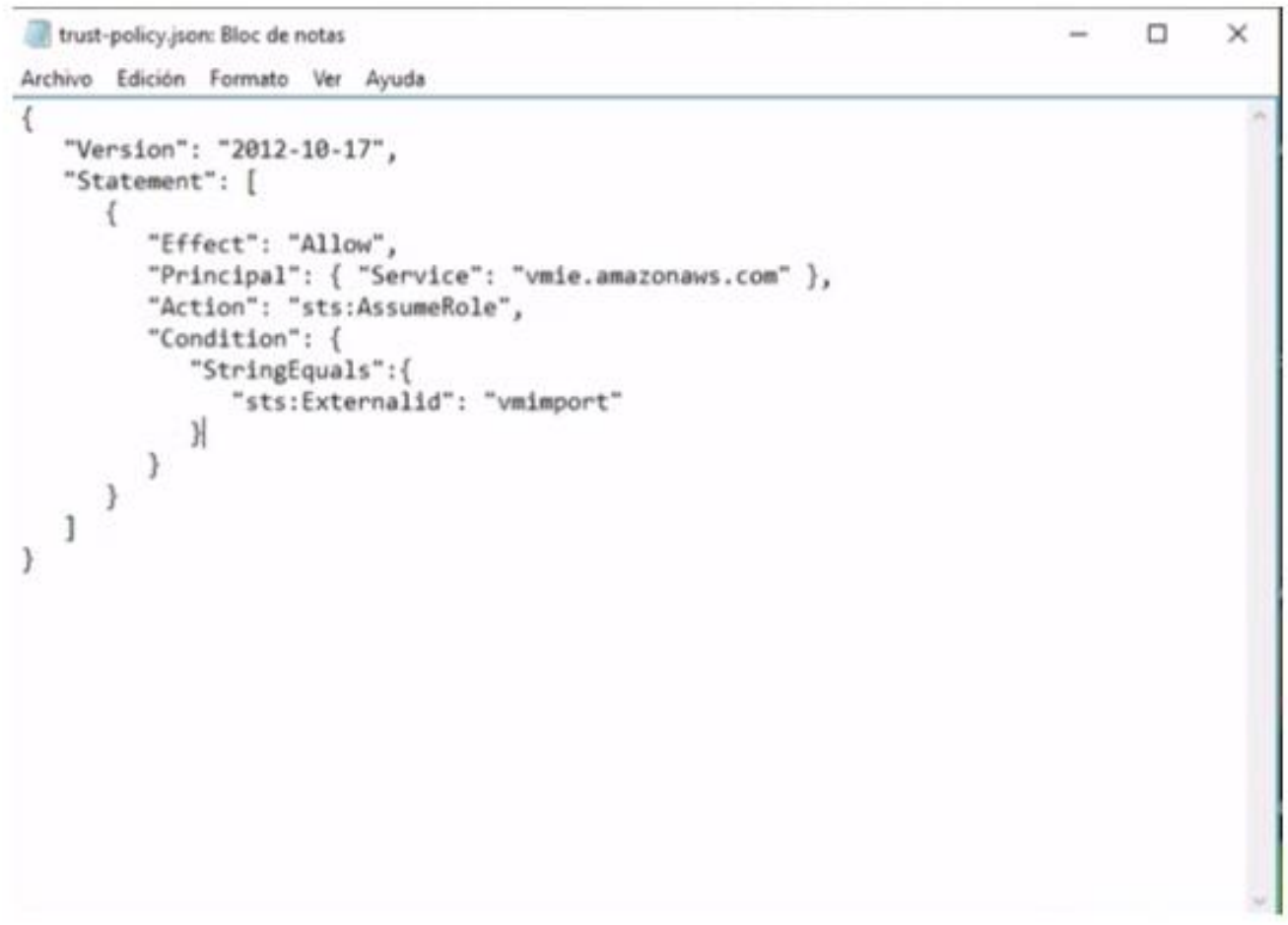

- También se creó el archivo role-policy.json que permitirá asignar la política al rol creado anteriormente.

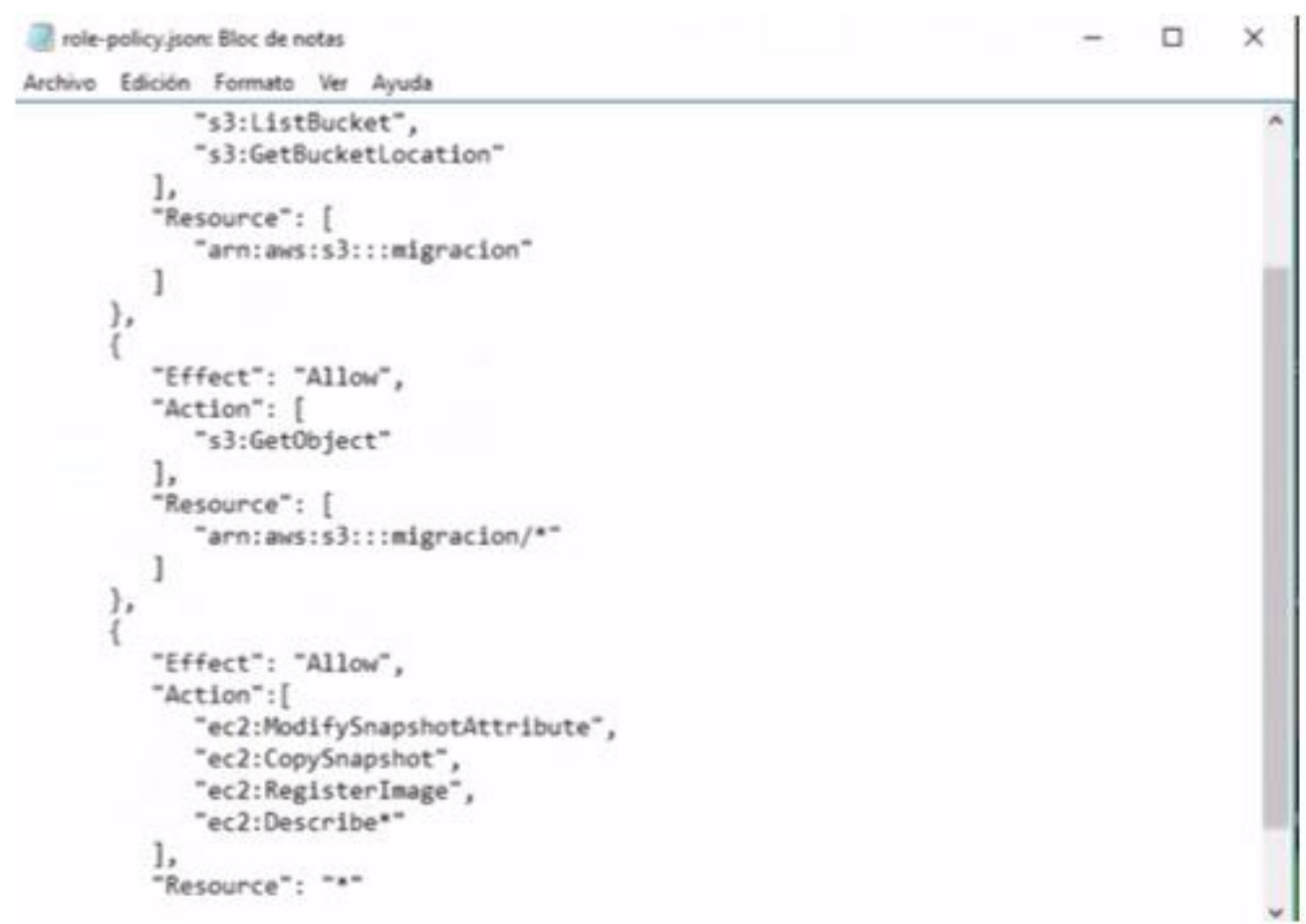

- Se creó el archivo containers. json el cual tiene las rutas desde donde se importará la VM. 


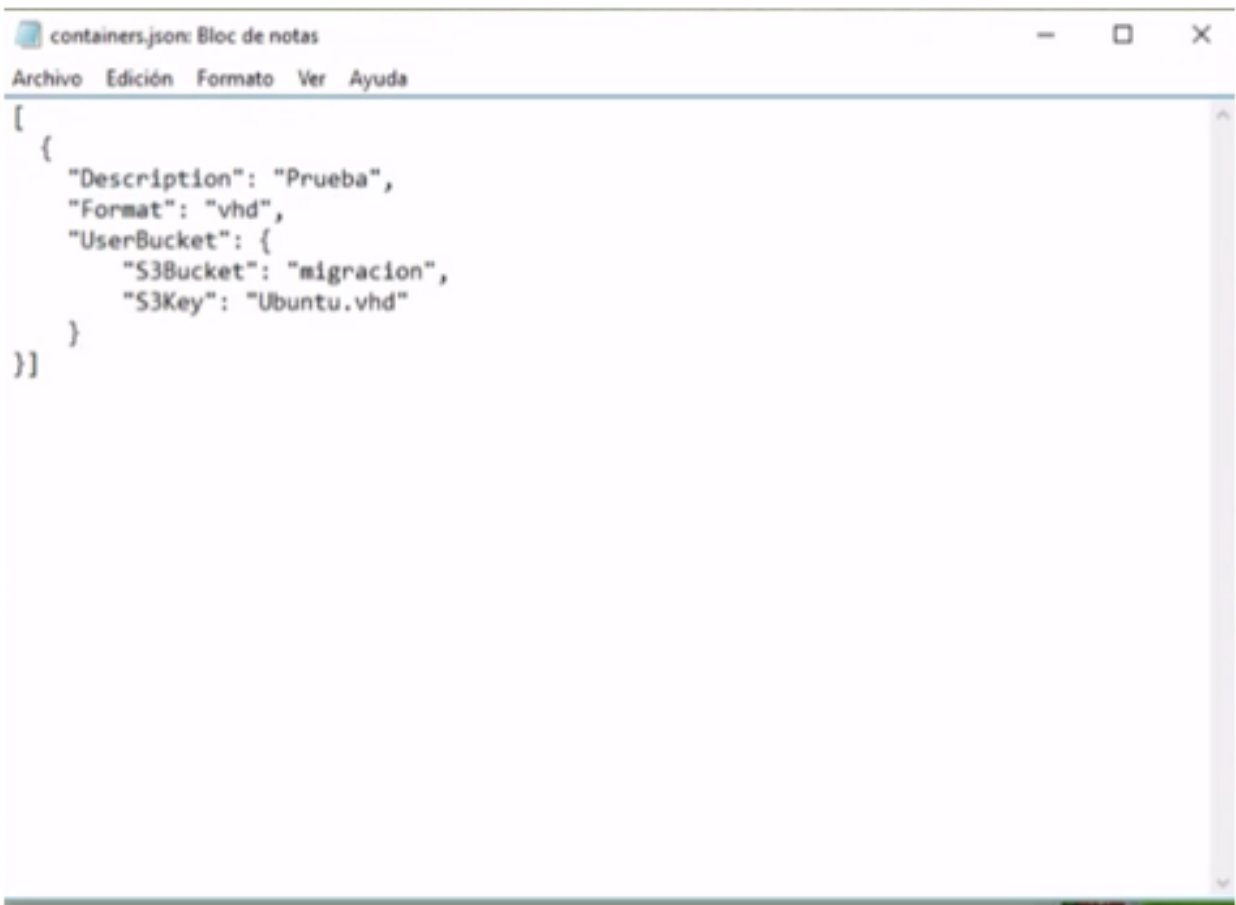

- Se debe ingresar el comando aws iam créate role -role-name [nombreRol] assume-role-policy-document file://[rutaArchivoTrust-Policy.json]

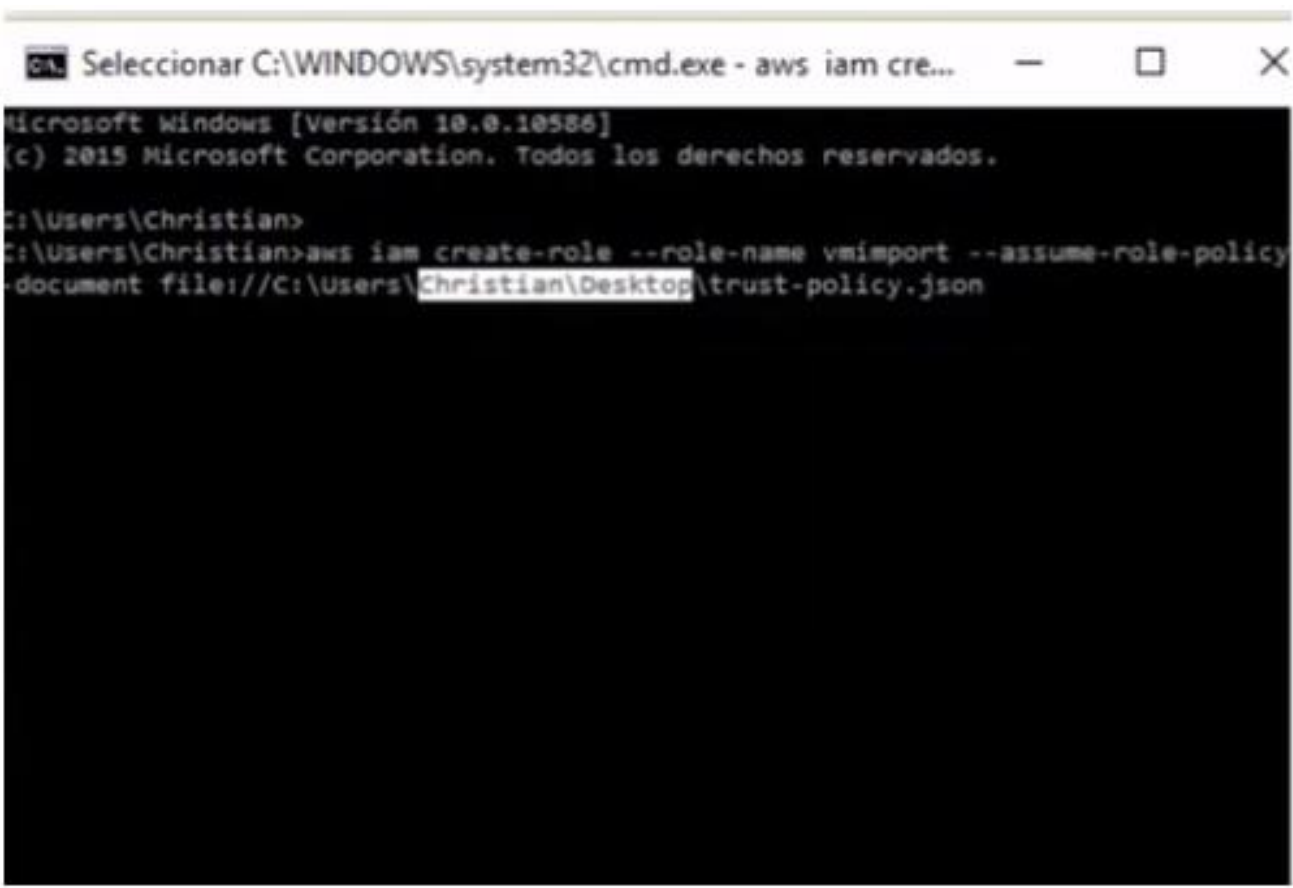

- Se recibirá una respuesta del contenido del archivo .json en el cmd 


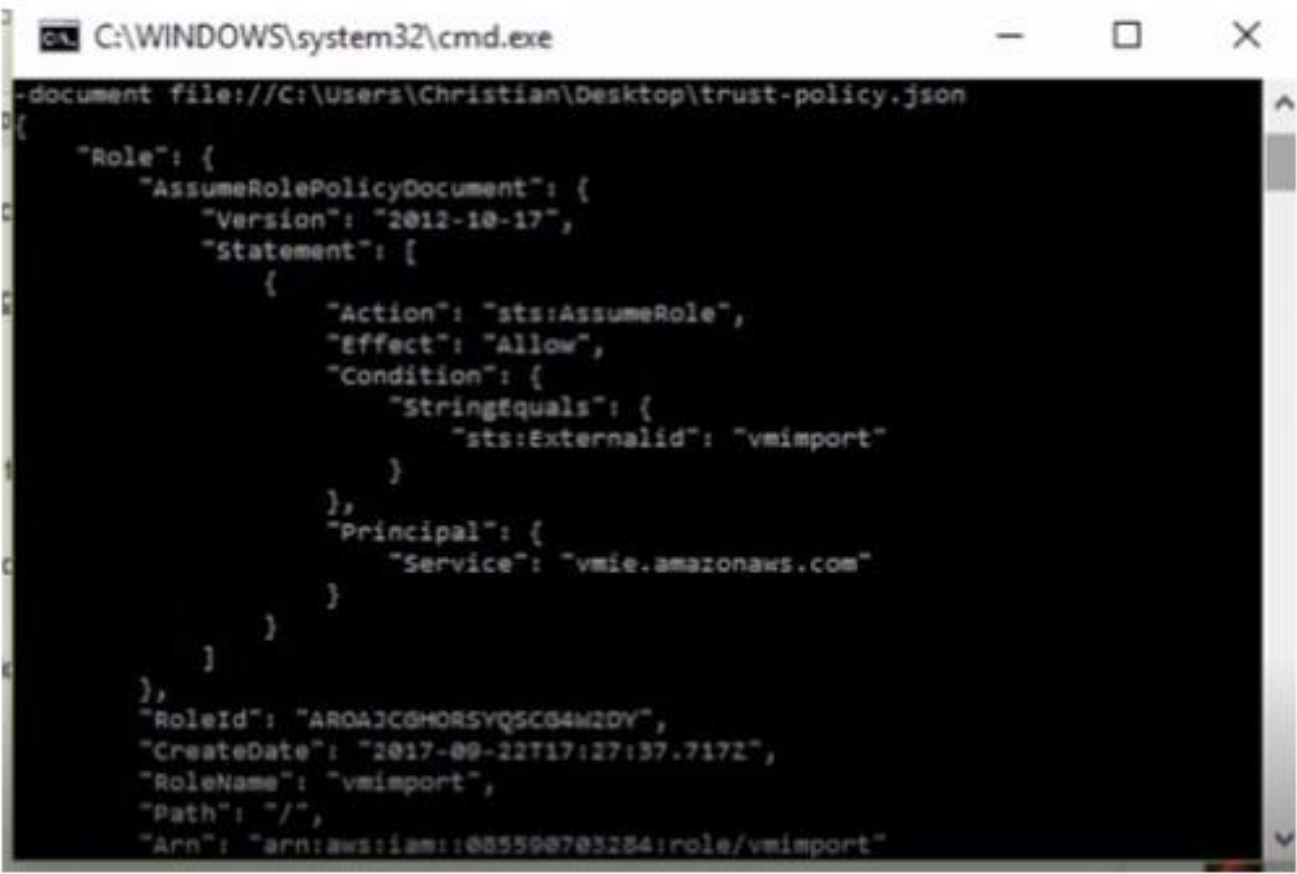

- En caso se desee, se puede ingresar a la plataforma EC2 de AWS para ver el rol creado

- Luego se debe ingresar el comando aws iam put-role-policy-role-name [nombreRol] -policy-name [nombrePolitica]-policy-document file://[rutaArchivoRole-Poliy.json]

C:IWINDOWSIsystem321 cmd.exe - aws iam put-role-policy... - $\square \times$

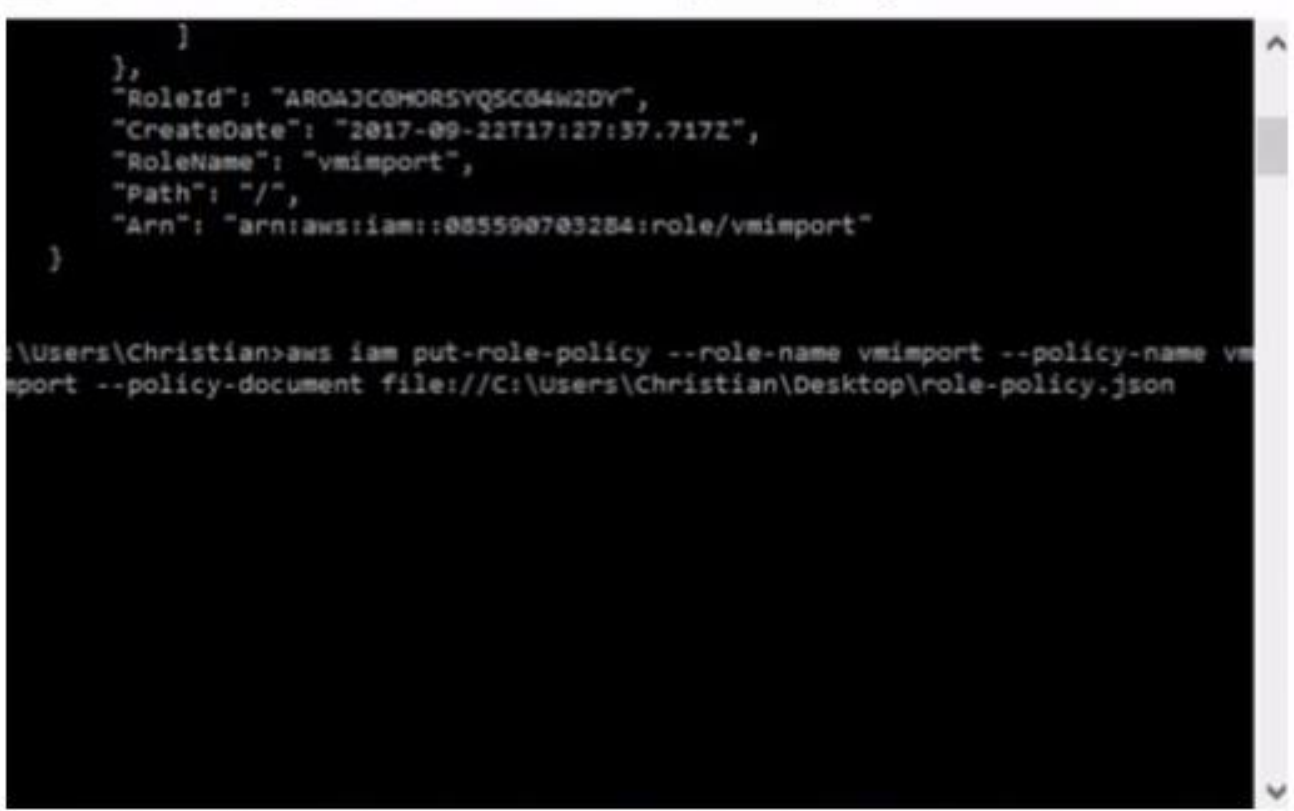

- Se creará la política que brinda acceso a los buckets (storage de aws) al servicio de importación

- A continuación se ingresó el siguiente comando para subir el archivo de disco duro al storage de la plataforma cloud: aws $\mathbf{s 3} \mathrm{cp}$ [RutaArchivoDiscoDuro] s3://[bucket] 


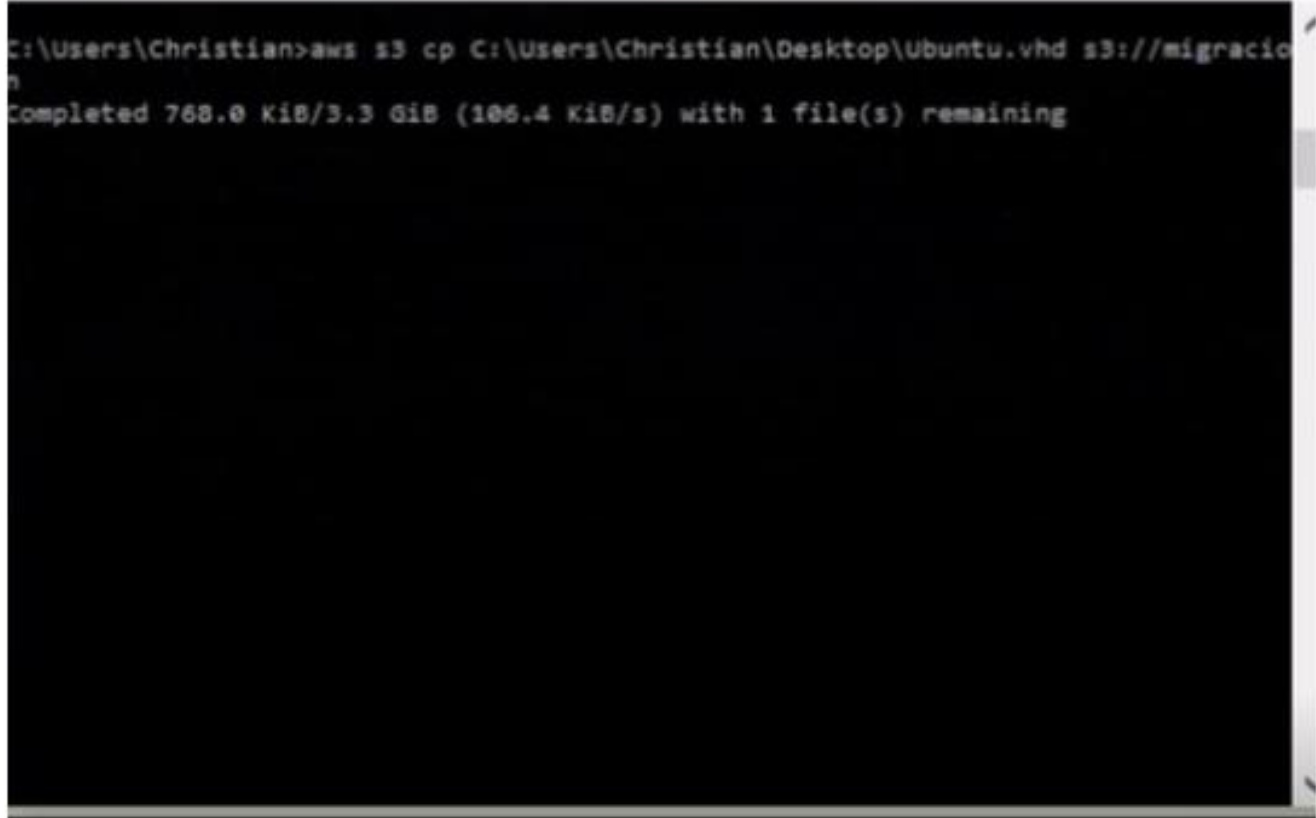

- Al finalizar se puede revisar que el archivo se encuentre en el bucket respectivo

- Luego se ingresó el comando ec2 import-image -description [Descripción] License-type [tipoLicencia (para este caso se usó BYOL, es decir, usará las licencias que ya poseo)]-disk-containers file://[rutaArchivoContainers.json]

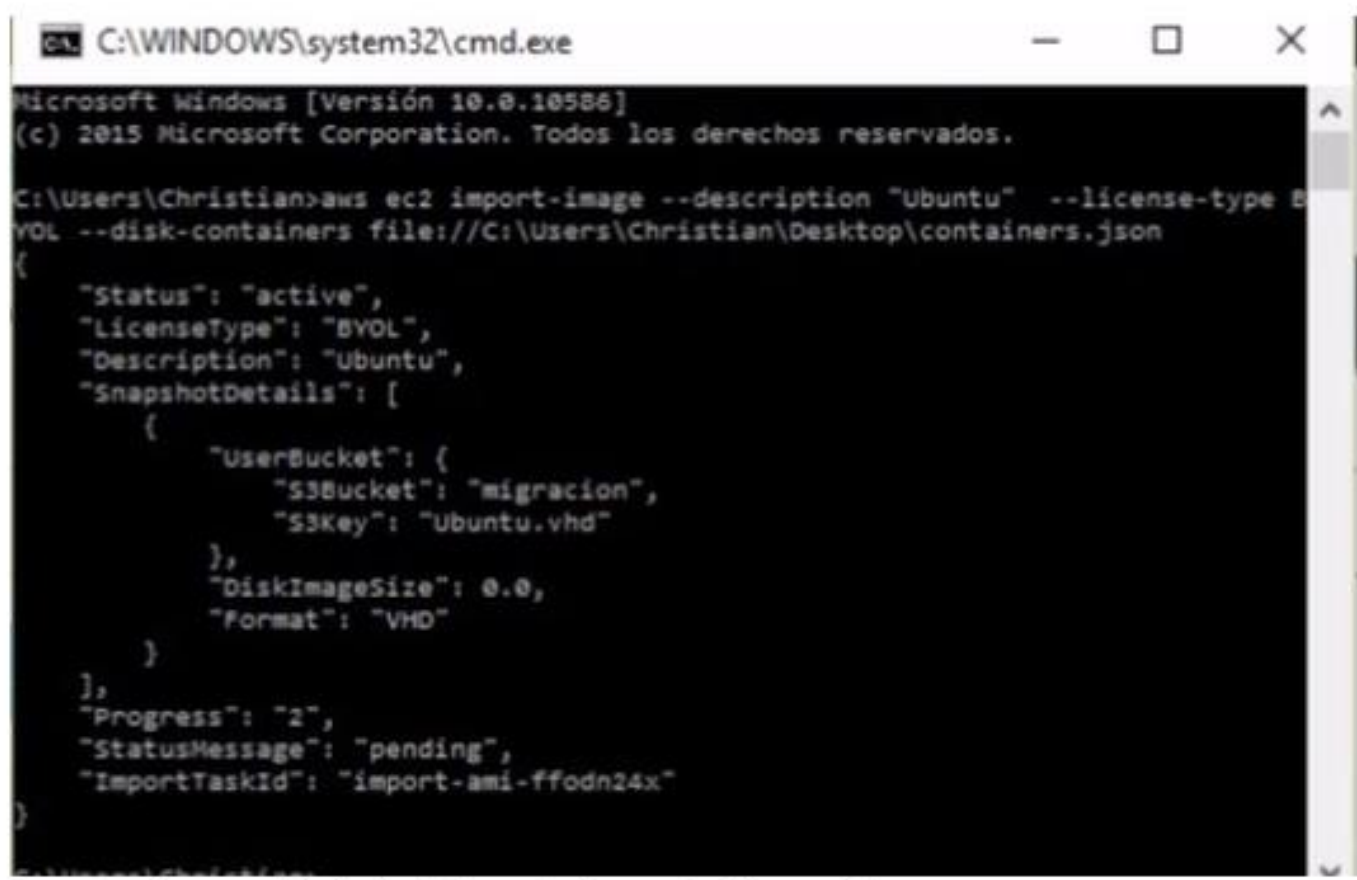

- Devolverá un resultado de la tarea de importación y un id

- Al finalizar nos dirigimos a EC2 en AWS y seleccionamos Launch instance y buscamos My AMI

- Ahí se verá la VM que importe, debo seleccionarla y proceder a asignar los recursos 


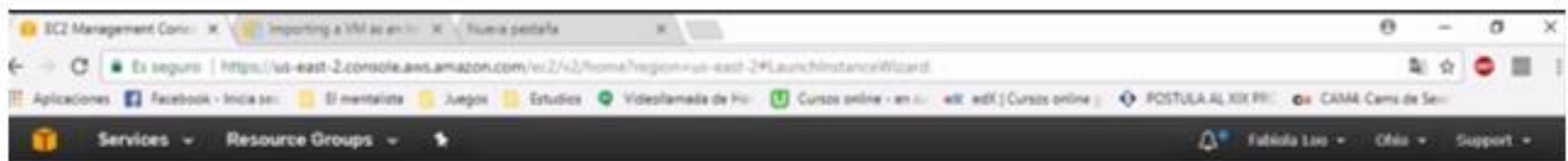

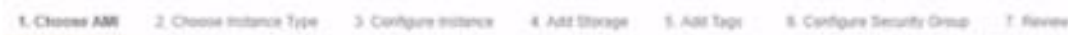

Step 1: Choose an Amazon Machine Image (AMI) cancew ans Exin

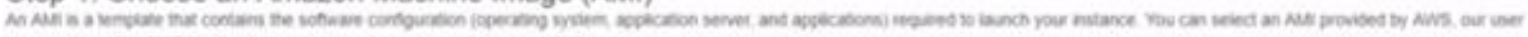

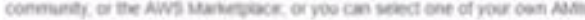

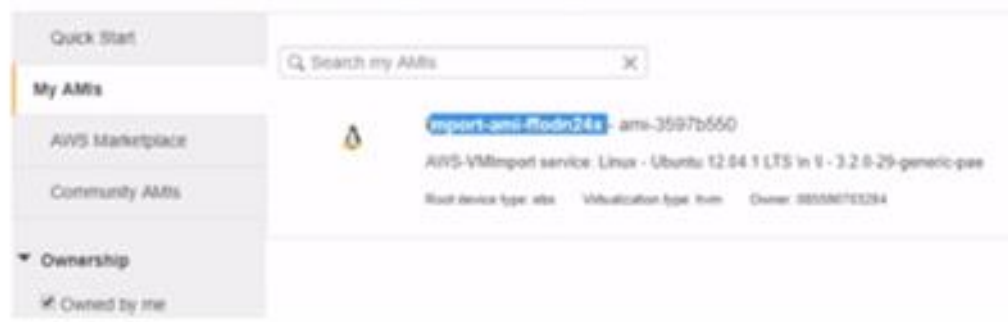

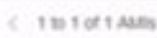

\begin{tabular}{|c|}
\hline Byani \\
\hline Ans Maniveas: \\
\hline Coemunty nos \\
\hline $\begin{array}{l}\text { Omearsivg } \\
\text { connes by ine }\end{array}$ \\
\hline
\end{tabular}

- Se procede a asignar los recursos de disco duro, grupos de seguridad, etiquetas, reglas de entrada/salida, etc. Al finalizar se notificará la correcta creación de la vm.|

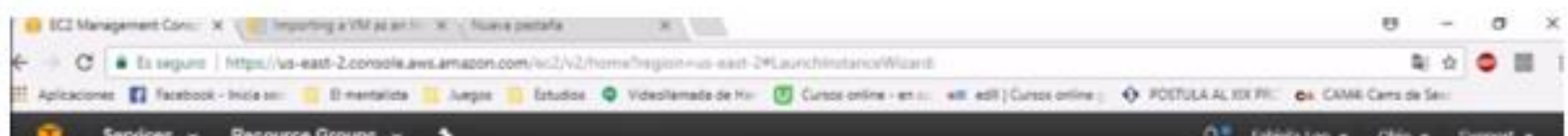

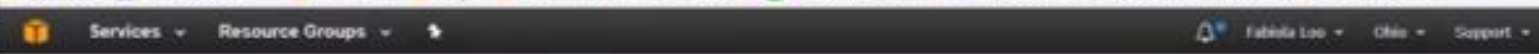

Launch Status

- Your instances are now launching

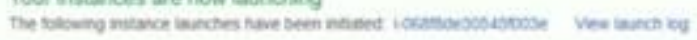

9 Get noified of estimated charges

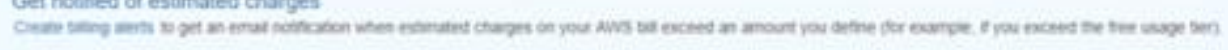

How to connect to your instances

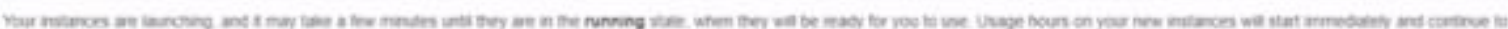

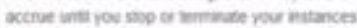

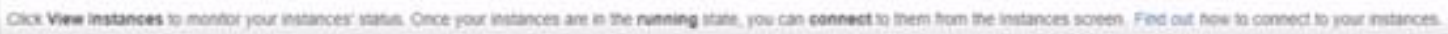

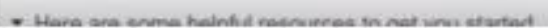

- Para poder conectarnos a la VM accedemos a PuTTY e ingresar en Host lo siguiente: usuario@[ip/host] 


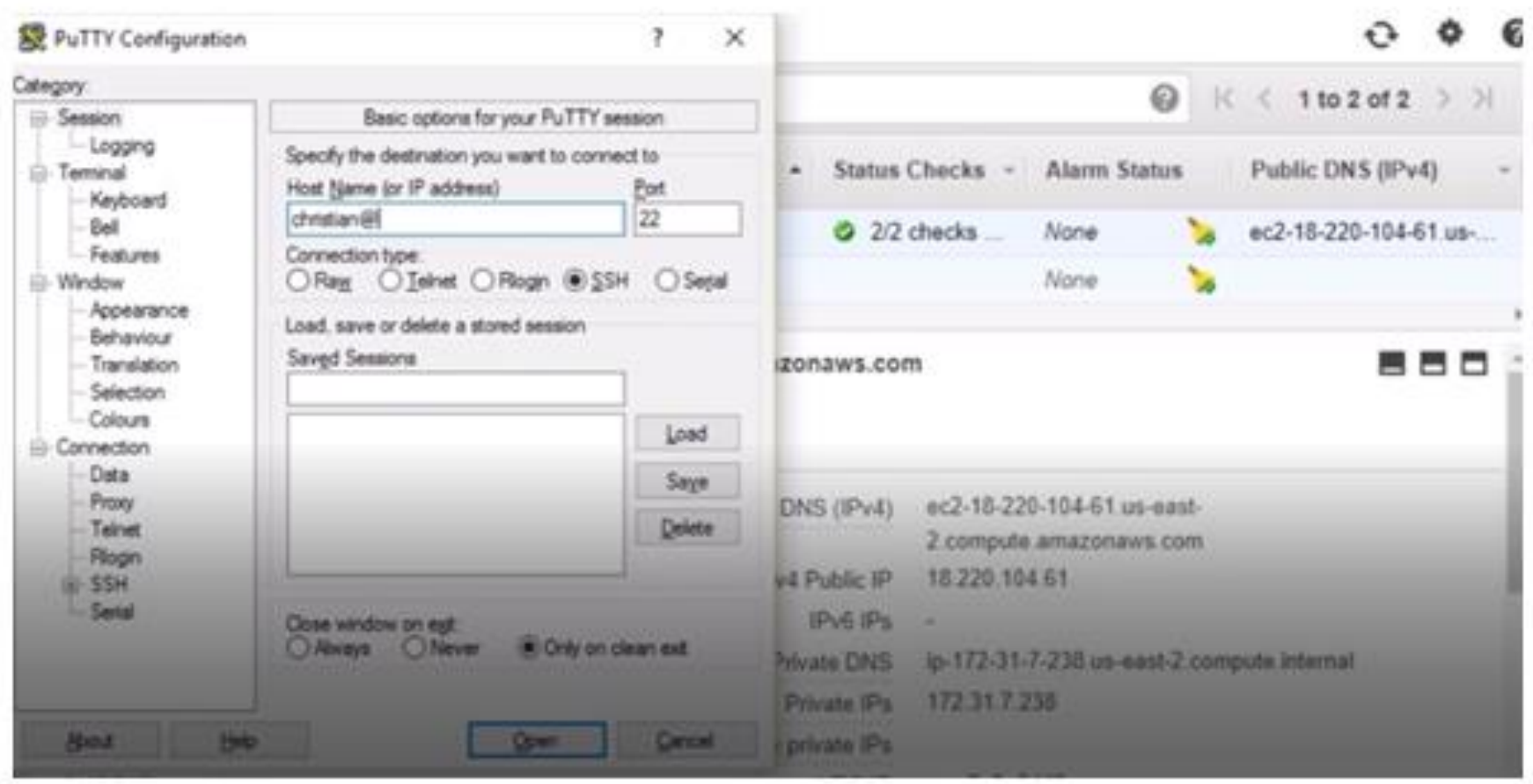

- Luego se ingresa la clave del usuario y se habrá accedido a la vm en cloud.

G9. ec2-18-220-104-61.us-east-2.computeamazonaws.com - PuTTY

Tsing username "christan".

chriatianfec2-18-220-104-61. us-east-2. compute. amazonaws . com " g pasatord:

Welcome to Jbuncu 12.04 .1 LTS (GarV/Linux 3.2.0-29-gener1c-pae 1686)

* Documentation: hteps://help.ubuntu.com/

624 packages can be updated.

934 updates are security updates.

New release '14.04.5 trs' avallable.

Run 'do-release-upgrade' to upgrade to it.

Last login: Thu Sep 28 18:26:39 2017 fxom 190.113.208.79 Bysq1

- Se puede acceder a MySQL para ver la bd y hacer consultas: 


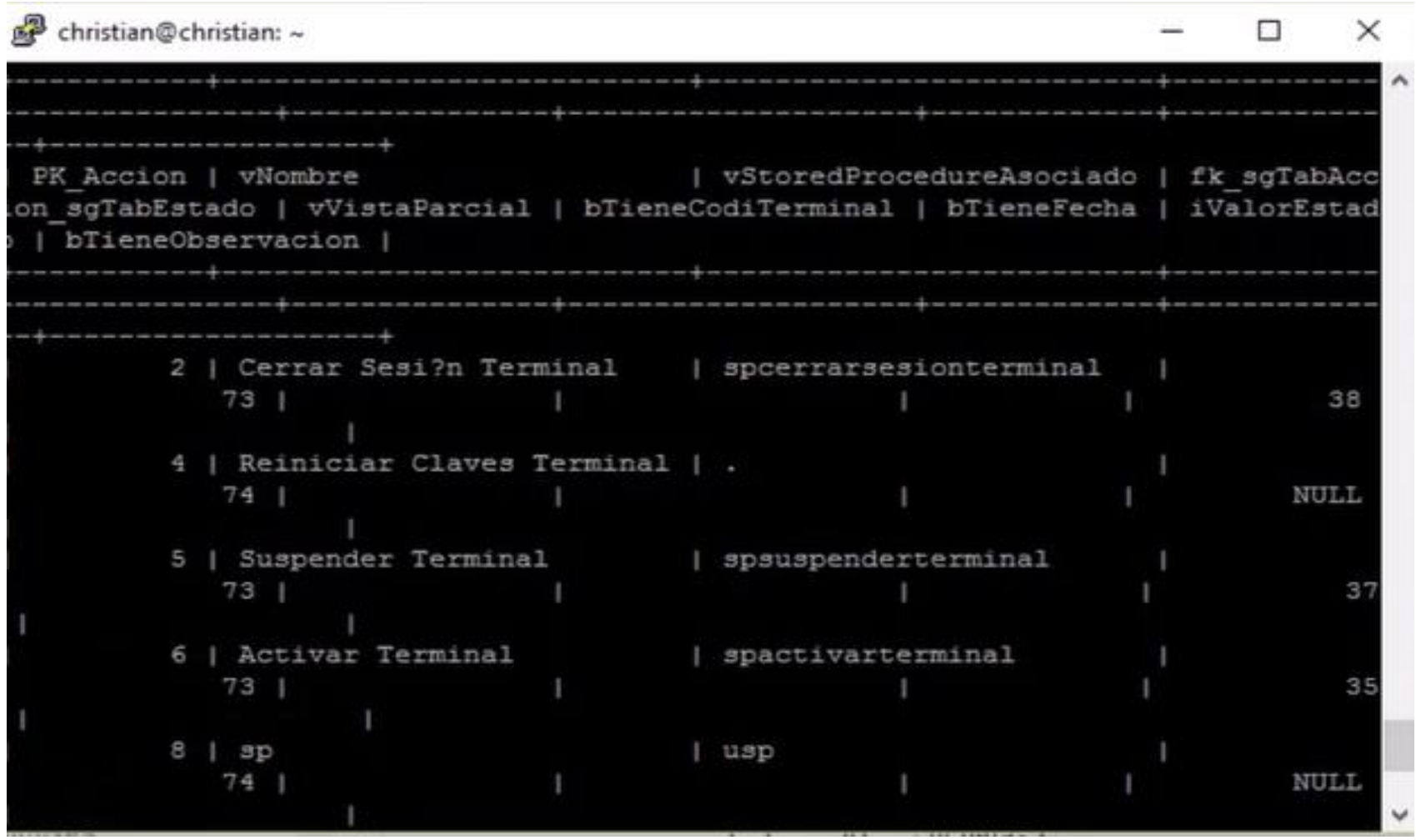

B christian@christian:
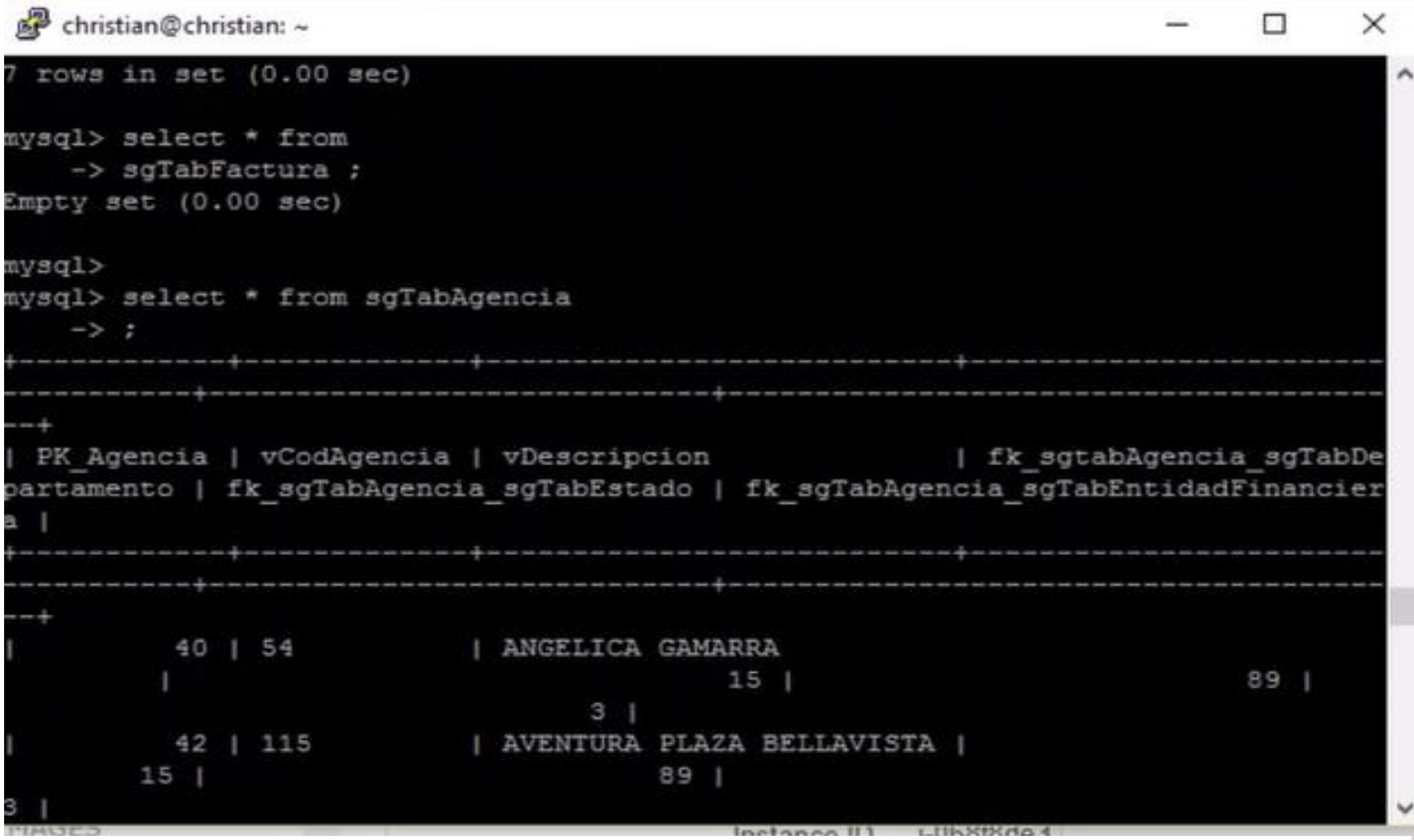

\section{CONCLUSIONES}

Finalizando con la implementación, se concluye que se logró migrar correctamente el servidor. 


\section{VALIDACION DE EXPERTO \\ Modelo de Migración a la nube de los servidores de una data Center}

Experto: Juan Manuel Cuya Cabanillas

PUNTUACION: 94

No cumple

012345678910

Cumple Satisfactoriamente

\begin{tabular}{|c|c|c|}
\hline$\#$ & CRITERIOS & PUNTUACION \\
\hline 1 & Flexibilidad & 9 \\
\hline 2 & Escalabilidad & 9 \\
\hline 3 & Seguridad & 10 \\
\hline 4 & Mejora Continua & 9 \\
\hline 5 & Reducción de Riesgos & 9 \\
\hline 6 & Facilidad de uso & 10 \\
\hline 7 & Reducción de Costos & 10 \\
\hline 8 & Administrable & 9 \\
\hline 9 & Servicio Medible & 9 \\
\hline \multirow[t]{2}{*}{10} & Monitoreo & 10 \\
\hline & TOTAL & 94 \\
\hline
\end{tabular}

La técnica utilizada ha sido NPS (Net Promoter Score) dando como resultado 94\%

A la pregunta si el cliente experto recomendaría nuestro proyecto a otras empresas ha sido satisfactorio. 
ANEXO 14: ACTA DE APROBACIÓN DEL OBJETIVO

1 


\begin{tabular}{|l|l|r|l|l|}
\hline Proyecto: & $\begin{array}{r}\text { Modelo de migración a la } \\
\text { nube de los servidores de un } \\
\text { data center }\end{array}$ & & \\
\hline & & $\mathbf{N}^{*}:$ & 1 \\
\hline
\end{tabular}

\section{Acta de Aprobación $\mathrm{N}^{\circ} 001$}

\section{Sección 1. Información General}

\begin{tabular}{|c|c|c|c|}
\hline Código y Nombre del Proyecto & Fecha de reunión & Hora inicio & Hora fin \\
\hline $\begin{array}{l}\text { MMNSDC - Modelo de migración a la nube de los } \\
\text { servidores de un data center. }\end{array}$ & $01 / 07 / 2017$ & $18: 00$ & $21: 00$ \\
\hline \multicolumn{4}{|l|}{ Elaborado por: } \\
\hline \multicolumn{4}{|l|}{ Fabiola Loo Cuya } \\
\hline Asistentes & Cargo & & Asistió \\
\hline Edgar Diaz Amaya & Profesor Cliente & & $x$ \\
\hline Fabiola Loo Cuya & Jefe de Proyecto & & $\mathrm{x}$ \\
\hline Christian Rojas Solórzano & Jefe de Proyecto & & $x$ \\
\hline
\end{tabular}

\section{Sección 1. Acuerdos}

\section{\begin{tabular}{l|l}
\hline Nro, Acuerdo & Acuerdo
\end{tabular}}

1 Aprobación del Objetivo 1: Documento de Buenas prácticas para la migración a la nube

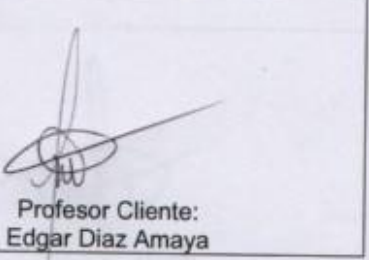

Página 1 de 1 


\section{ANEXO 15: ACTA DE APROBACIÓN DEL OBJETIVO \\ 2}




\begin{tabular}{|l|l|r|l|l|}
\hline Proyecto: & $\begin{array}{r}\text { Modelo de migración a la } \\
\text { nube de los servidores de un } \\
\text { data center }\end{array}$ & & \\
\hline & & & $\mathbf{N}^{*}:$ & 2 \\
\hline
\end{tabular}

\section{Acta de Aprobación Nº02}

\section{Sección 1. Información General}

\section{Código y Nombre del Proyecto}

MMNSDC - Modelo de migración a la nube de los

servidores de un data center.

Fecha de reunión

Hora inicio

$08 / 07 / 2017$

18:00

Hora fin

Elaborado por:

Fabiola Loo Cuya

Asistentes

Cargo

Asistio

Edgar Diaz Amaya

Fabiola Loo Cuya

Christian Rojas Solórzano

Profesor Cliente

Jefe de Proyecto

Jefe de Proyecto

$x$

x

\section{Sección 1. Acuerdos}

\section{Nro. Acuerdo Acuerdo}

1

Aprobación del Objetivo 2: Diseño del modelo de migración a la nube de los servidores de un data center

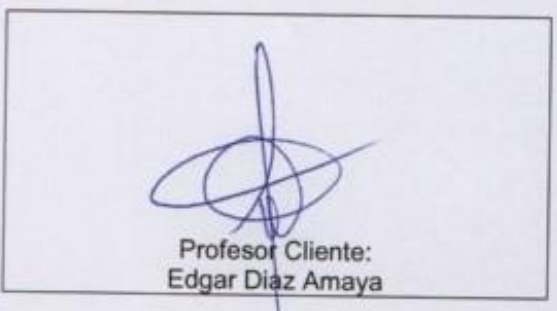

Página 1 de 1 


\section{ANEXO 16: ACTA DE APROBACIÓN DEL OBJETIVO 3}

ACTA DEL PROFESOR CLIENTE 


\begin{tabular}{|l|l|l|l|l|}
\hline Proyecto: & $\begin{array}{l}\text { Modelo de Migración a la nube de } \\
\text { los servidores de un Data Center } \\
\text { Tier } 1\end{array}$ & $\mathbf{N}^{\circ}$ & 1 \\
\hline Fecha: & $25 / 09 / 2017$ & \\
\hline
\end{tabular}

\section{Acta de Aprobación Objetivo 3}

\section{Sección 1. Información General}

\begin{tabular}{|c|c|c|c|}
\hline Codligo y Nombre del Rroyecto & Fecha de reunión. & Hora inicio & Hora fin \\
\hline $\begin{array}{l}\text { Modelo de Migración a la nube de los servidores de un } \\
\text { Data Center - MMNSDC }\end{array}$ & $25 / 09 / 2017$ & $18: 30$ & $19: 00$ \\
\hline Elaborado por: & & & \\
\hline \multicolumn{4}{|l|}{ Fabiola Loo } \\
\hline Asistentes & Cargo & & Asistio \\
\hline Edgar Diaz & Profesor Cliente & & $x$ \\
\hline Fabiola Loo & Jefe de Proyecto & & $x$ \\
\hline Christian Rojas & Jefe de Proyecto & & $x$ \\
\hline
\end{tabular}

\section{Sección 2. Acuerdos}

\begin{tabular}{|c|l|}
\hline Nro. Acuerdo & Acuerdo \\
\hline 1 & $\begin{array}{l}\text { Aprobación del objetivo 3: validación del modelo de migración a la nube de los servidores de un } \\
\text { datacenter. }\end{array}$ \\
\hline
\end{tabular}

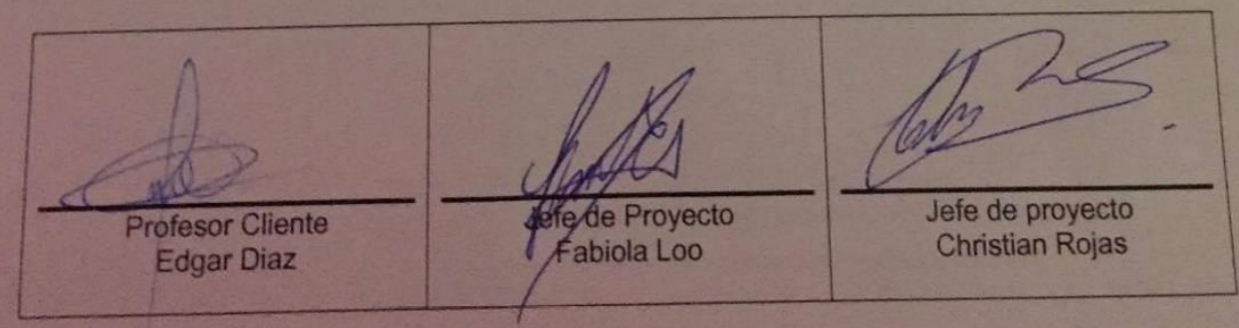

ACTA DE LA EMPRESA CLIENTE 


\begin{tabular}{|l|l|l|l|l|}
\hline Provecto: & $\begin{array}{l}\text { Modelo de Migracion a la nube de } \\
\text { los servidores de un Data Centor }\end{array}$ & & \\
\hline Fecha: & $22 / 09 / 2017$ & $N^{*}$ & 1 \\
\hline
\end{tabular}

\section{Acta de Aprobación}

\section{Sección 1. Información General}

\begin{tabular}{|c|c|c|c|}
\hline Codiga y Nombre del Proyecto & Fecha de reunion & Horalinicio & Hora fin \\
\hline $\begin{array}{l}\text { Modelo de Migración a la nube de los servidores de un } \\
\text { Data Center - MMNSDC }\end{array}$ & $22 / 09 / 2017$ & 19.30 & \\
\hline
\end{tabular}

\begin{tabular}{|c|c|c|}
\hline Fabiola Loo & & \\
\hline Asistentes & Cargo & Asistio \\
\hline Jimmy Novoa & $\begin{array}{l}\text { Jefe de proyectos } \\
\text { (PMO) }\end{array}$ & $x$ \\
\hline Fabiola Loo & Jefe de Proyecto & $x$ \\
\hline Christian-Rojas & Jefe de Proyecto & $x$ \\
\hline
\end{tabular}

\section{Sección 2. Acuerdos}

\begin{tabular}{|cl}
\hline Nros Acuerdo & Acuerdo \\
\hline 1 & $\begin{array}{l}\text { Se valido el modelo de migracion a la nube de los servidores de un data center en la empresa } \\
\text { Globokas Peru. }\end{array}$ \\
2 & El modelo se ejecuto en un plazo de 2 dias
\end{tabular}

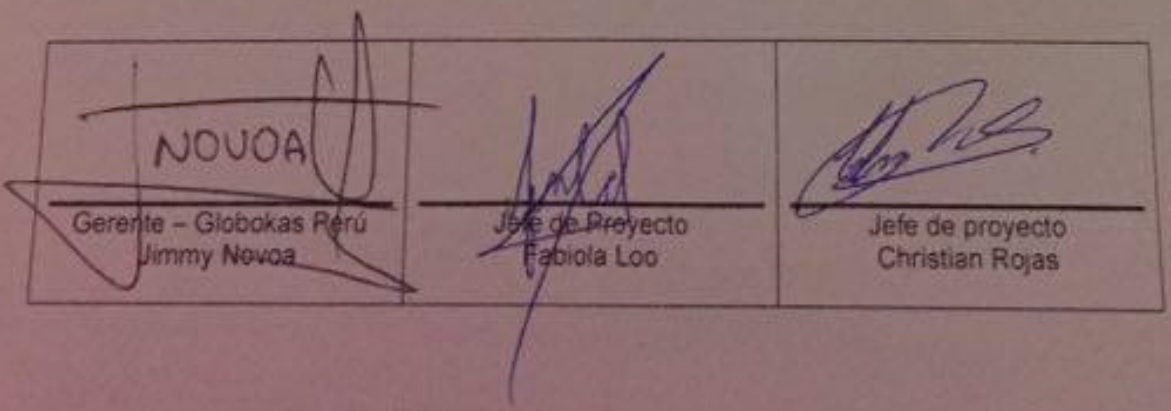


ACTA DE JUICIO DE EXPERTOS

\begin{tabular}{|l|l|l|l|l|}
\hline Proyecto: & $\begin{array}{l}\text { MODELO DE MIGRACIÓN A LA } \\
\text { NUBE DE LOS SERVIDORES DE UN } \\
\text { DATA CENTER }\end{array}$ & & \\
\hline & Fecha: & $02 / 11 / 2017$ & $\mathbf{N}^{*}:$ & 3 \\
\hline
\end{tabular}

\section{Acta de Validación}

Sección 1. Información General

\begin{tabular}{|c|c|c|c|}
\hline Código y Nombre del Proyecto & Fecha de reunión & Hora inicio & Hora fin \\
\hline $\begin{array}{l}\text { MMNSDC - Modelo de Migración a la Nube de los } \\
\text { Servidores de un Data Center }\end{array}$ & $02 / 11 / 2017$ & & \\
\hline \multicolumn{4}{|l|}{ Elaborado por: } \\
\hline \multicolumn{4}{|l|}{ Fabiola Loo/Christian Rojas } \\
\hline Asistentes & Cargo & & Asistió \\
\hline Juan Manuel Cuya & Experto Cloud & & $\mathrm{x}$ \\
\hline Fabiola Loo & Alumno & & $x$ \\
\hline Christian Rojas & Alumno & & $x$ \\
\hline
\end{tabular}

Sección 1. Acuerdos

Nro. Acuerdo Acuerdo

1 Se validó el modelo de migración a la nube de los servidores de un data center, con juicio experto

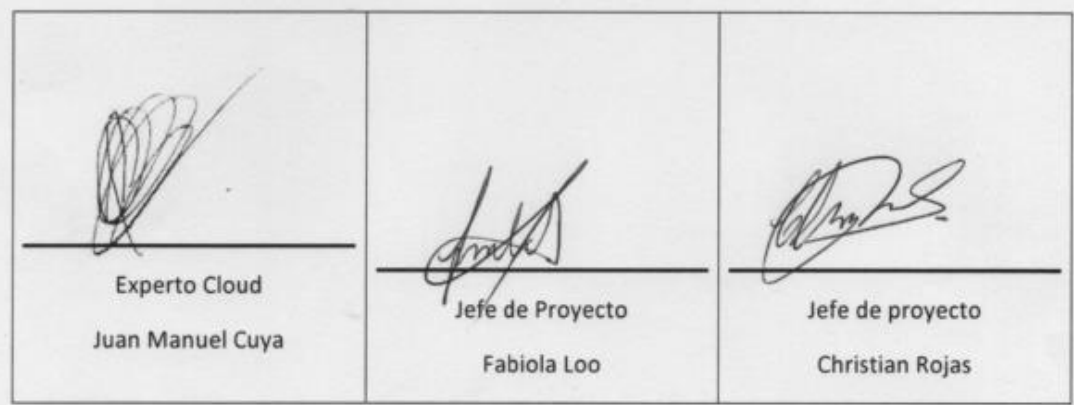




\section{ANEXO 17: ACTA DE APROBACIÓN DEL OBJETIVO 4}




\begin{tabular}{|l|l|l|l|l|}
\hline Proyecto: & $\begin{array}{l}\text { MODELO DE MIGRACIÓN A LA } \\
\text { NUBE DE LOS SERVIDORES DE UN } \\
\text { DATA CENTER }\end{array}$ & & \\
\hline Fecha: & $11 / 11 / 2017$ & $N^{*}:$ & 4 \\
\hline
\end{tabular}

\section{Acta de Aprobación Objetivo 4}

\section{Sección 1. Información General}

\begin{tabular}{|c|c|c|c|}
\hline Codigo y Nombre del Proyecto & Fecha de reunión & Hora inicio & Hora fin \\
\hline $\begin{array}{l}\text { MMNSOC - Modelo de Migración a la Nube de los } \\
\text { Servidores de un Data Center }\end{array}$ & $11 / 11 / 2017$ & 10.00 & 11.00 \\
\hline \multicolumn{4}{|l|}{ Elaborado por: } \\
\hline \multicolumn{4}{|l|}{ Fabiola Loo/Christian Rojas } \\
\hline Asistentes & Cargo & & Asistio \\
\hline Edgar Diaz & Profesor Cliente & & $x$ \\
\hline Fabiola Loo & Alumno & & $x$ \\
\hline Christian Rojas & Alumno & & $x$ \\
\hline
\end{tabular}

\section{Sección 4. Acuerdos}

\section{Nro. Acuerdo Acuerdo}

Aprobación del objetivo 4: Proponer un plan de continuidad que garantice la continuidad del proyecto.

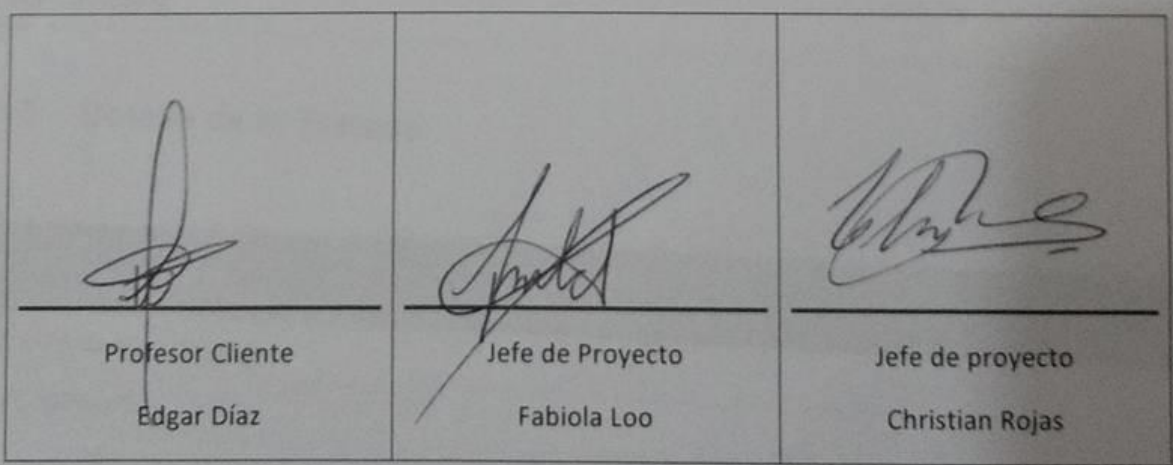




\section{ANEXO 18: PLAN DE CONTINUIDAD}

\section{PLAN DE CONTINUIDAD}

Para garantizar la continuidad del proyecto, se tomaron ciertas medidas, las medidas del plan de continuidad del presente proyecto son:

1. Capacitaciones

2. Acuerdos a nivel de servicios

3. Back ups y DRP Cloud

4. Soporte

A continuación se detallará en que consiste cada una de las medidas consideradas:

1. Capacitación: Se realizó la capacitación del personal que trabaja directamente con los servicios cloud, como los servidores virtuales migrados. En la capacitación se tocaron los siguientes temas:

- Imágenes e instancias en AWS

- Configuración de características de instancias

- Monitoreo de los servidores en cloud

- Conexión remota a AWS EC2

- Límites actuales de los servicios (storagel procesamiento, transferencia)

- Uso del soporte de AWS

- Facturación de AWS

Quienes asistieron a la capacitación fueron:

- Jefe de Infraestructura de TI

- Analista de Redes

- Operadores de soporte técnico

- Operadores de Sistemas

- Analista de Seguridad

La capacitación permitió explicar a los asistentes como es que funcionarán los servidores virtuales desde cloud y las actividades que requieren realizar ante algún inconveniente; asimismo se mostró que los procesos actuales no tendrían cambios drásticos a excepción del mantenimiento físico de los servidores y generación de back ups los cuales serán realizados por el proveedor (AWS).

Se requerirá que el personal de $\mathrm{Tl}$ siga siendo capacitado constantemente sobre los servicios cloud que utilizan, a fin de saber cómo llevar a cabo sus labores correctamente. Estas capacitaciones se deberán brindar con una frecuencia trimestral, debido a los cambios y nuevas funcionalidades que pueden surgir en ese lapso de tiempo.

En caso que un nuevo miembro ingrese al equipo que trabaja directamente con los servicios cloud, este deberá tener asignado un responsable que se encargue de capacitarlo para que su aprendizaje no se vea retenido hasta la próxima capacitación. Se deberá capacitar al nuevo miembro al menos en los puntos tratados en esta primera capacitación, los cuales se pueden ver al principio. 
Los objetivos para las capacitaciones futuras son las siguientes:

- Proveer conocimientos y desarrollar habilidades que cubran la totalidad de requerimientos solicitados.

- Contribuir a elevar y mantener un buen nivel de eficiencia individual y rendimiento colectivo.

- Ayudar en la preparación de personal calificado, acorde con los planes, objetivos y requerimientos de la Empresa.

- Apoyar la continuidad y desarrollo de las operaciones.

2. Acuerdos a nivel de servicios: Se estableció un acuerdo a nivel de servicio de disponibilidad con el proveedor de la plataforma cloud (AWS), en dicho acuerdo el proveedor nos garantiza la disponibilidad de los servidores virtuales migrados de al menos $99.95 \%$ cada mes (tiempo de no disponibilidad menor a 22 minutos al mes). En caso el proveedor incumpla con este acuerdo, se compromete a brindar un porcentaje del crédito del monto facturado, según la siguiente tabla:

Tabla 1: Porcentajes de tiempo

\begin{tabular}{|l|l|}
\hline $\begin{array}{l}\text { Porcentaje de tiempo de actividad } \\
\text { mensual }\end{array}$ & $\begin{array}{l}\text { Porcentaje de crédito del } \\
\text { servicio }\end{array}$ \\
\hline $\begin{array}{l}\text { Mayor o igual a } 99 \% \text { pero menor que } \\
\text { 99.95\% }\end{array}$ & $10 \%$ \\
\hline \begin{tabular}{l} 
Menor a 99\% \\
\hline
\end{tabular} & $30 \%$ \\
\hline
\end{tabular}

En caso que la disponibilidad del servicio sea menor al estipulado, se presentará una solicitud al proveedor en el cual se reclama el porcentaje del crédito correspondiente, el cual debe ser lo más detallado posible (indicar ID de servicios que no estuvieron disponibles entre los rangos permitidos) para que el proveedor revise el caso.

Para detalles adicionales del acuerdo se pueden revisar en el siguiente link del proveedor: $\quad$ https://d1.aw5static.com/legal/amazon-ec2sla/Amazon EC2 SLA ES.1400e34590a03289db0473094585ed24d18ba2d6.pdf

3. Backs up y DRP Cloud: Se estableció con la plataforma cloud las peliticas de respaldo, en la cual se detalla lo siguiente:

- Los back ups de los servidores migrados se realizan en automático, siendo los de tipo full cada semana y de tipo incremental cada 3 días.

- Se habilitará la opción de tail-log para los back ups

- Cada respaldo que se realice dejará LOGS en el mismo servidor y en uno específico para auditorias. 
- Los respaldos realizados serán probados luego de su creación para corroborar su fiabilidad y seguridad.

- Se notificará al Jefe de Infraestructura de TI por correo cuando a fin de mes de los respaldos realizados.

- Los respaldos podrán ser destruidos luego de 5 años de creados.

Por otro lado, se debe incluir los servidores migrados en el plan de recuperación de desastres del proveedor de servicios cloud, para que en caso el centro de datos donde se encuentre los servidores virtuales resulte dañado, se pueda levantar los servicios desde otro centro de datos que sea un reflejo del primero.

A cambio de estos servicios la empresa se compromete a pagar al proveedor cloud la suma de $\$ 0.0125$ por cada GB de back up que se almacene cada mes.

4. Soporte: Finalmente, se cuenta con un equipo que brinda soporte a los usuarios en caso de incidentes no previstos, para este punto se preparó el documento "Plan de Soporte" en el cual se pueden encontrar más detalles. La persona encargada del soporte, además de tener vocación por el servicio a los demás, debe de saber cómo mínimo, adicionalmente a lo tratado en la capacitación mencionada, lo siguiente:

- Virtualización

- Administración de sistemas operativos

- Administración de redes

- Infraestructura de TI

- ITIL 


\section{ANEXO 19: PLAN DE SOPORTE}

\section{PLAN DE SOPORTE}

El presente documento contiene el plan de continuidad para el proyecto Modelo de migración a la nube de los servidores de un data center. El siguiente contiene un conjunto de tareas que permite a la empresa continuar sus actividades en la situación de que un evento afecte sus operaciones. Este plan afecta tanto a los sistemas de información como al resto de procesos de una organización y tiene en cuenta la situación antes, durante y después de un incidente.

\section{OBJETIVO}

El objetivo del plan de continuidad de negocio es brindar una guía sobre la gestión operativa que se tendrá respecto a la propuesta realizada en el proyecto, abarcando aspectos tanto técnicos como de aplicación y brindando además una guía para la mitigación de eventualidades que puedan presentarse con el uso de la solución propuesta y con el paso del tiempo. Asimismo, brinda una guía respecto a la orientación a brindar hacia los usuarios finales y controla el registro de eventualidades para su posterior resolución.

Los procesos, actividades y tareas planteadas en los puntos subsiguientes se encuentran basados en estándares de aceptación mundial, dentro de los cuales destacamos el marco de referencia de ITIL, el cual es un conjunto de buenas prácticas para la gestión de servicios de tecnologías de Tl y permite abarcar de manera ideal el aspecto propio de soporte del sistema propuesto. En algunos casos se toma como referencia la ISO 22301 Continuidad de Negocio.

\section{OBJETIVOS ESPECÍFICOS}

Los objetivos específicos para el presente plan de continuidad de negocio son detallados a continuación:

- Permitir identificar planes de acción frente a contingencias

- Permitir identificar responsables frente a contingencias

- Permitir identificar acciones para la correcta operación del sistema a implementar

- Garantizar que el sistema esté operando adecuadamente

\section{BENEFICIOS}

Los beneficios de elaborar un plan de continuidad de negocio trascienden el esfuerzo que representa elaborarlo, es decir, resulta ventajoso emplear tiempo en la definición de las estrategias de operación y soporte por el beneficio que supone a futuro. A continuación, se mencionan los principales:

- Identificar y gestionar amenazas actuales y futuras para la organización.

- Adoptar un método proactivo para minimizar el impacto de los incidentes.

- Mantener al día y operar las funciones críticas durante los momentos de crisis.

- Minimizar los tiempos muertos durante los incidentes y mejorar el tiempo de recuperación.

- Demostrar seguridad a los clientes, proveedores y para las solicitudes de licitación. 
ROLES DE SOPORTE

Los roles que se han definido son los siguientes:

\begin{tabular}{|c|c|}
\hline Rol & Responsabilidad \\
\hline Administrador de $\mathrm{TI}$ & $\begin{array}{l}\text { - Identificar e integrar las tecnologías de información a los procesos } \\
\text { - Rel negocio con la finalidad de generar valor } \\
\text { - Anfraestructura tecnológica } \\
\text { - Asegurar que todas las actividades operativas cotidianas se lleven a } \\
\text { cabo puntual y confiablemente } \\
\text { - Asegurar la continuidad del negocio desarrollando controles y } \\
\text { planes de contingencia }\end{array}$ \\
\hline Gestor de Continuidad & $\begin{array}{l}\text { - Asegurar que los servicios de TI estén dispuestos para alcanzar los } \\
\text { niveles de disponibilidad esperados } \\
\text { - Crear y mantener el plan de disponibilidad }\end{array}$ \\
\hline Operador & $\begin{array}{l}\text { - Proveer el soporte de primer nivel } \\
\text { - Registrar y clasificar los incidentes reportados } \\
\text { - Ejecutar esfuerzos inmediatos para restaurar lo antes posible un } \\
\text { servicio de Tl que haya fallado }\end{array}$ \\
\hline Gestor de Incidentes & $\begin{array}{l}\text { - Monitorea la eficacia y eficiencia del proceso } \\
\text { - Controla el trabajo de los grupos de soporte } \\
\text { - Hace recomendaciones para la mejora del proceso } \\
\text { - Desarrolla y mantiene el sistema de gestión de incidentes }\end{array}$ \\
\hline Operador de soporte técnico & $\begin{array}{l}\text { - Proveer el soporte de segundo nivel } \\
\text { - Atender a consultas técnicas y dar servicio personalizado de forma } \\
\text { - } \text { Eroactiva a los clientes } \\
\text { - Ejecutar procedimientos de administración y mantenimiento }\end{array}$ \\
\hline Proveedor Cloud & $\begin{array}{l}\text { - Proveer el soporte de tercer nivel } \\
\text { - Se ocupa de que las aplicaciones y los sistemas que proveen la } \\
\text { funcionalidad necesaria para los servicios de Tl estén disponibles. } \\
\text { - Mantenimiento de aplicaciones personalizadas, así como la } \\
\text { personalización de productos }\end{array}$ \\
\hline
\end{tabular}

\section{PROCEDIMIENTO DE SOPORTE}

Empleando el modelo conceptual de ITIL para la óptima gestión del soporte brindado al proyecto propuesto, hemos identificado el siguiente flujo de soporte conforme sean las necesidades y los tiempos de respuesta: 
Tiempo de

respuesta y solución

5 a 15 minutos
Tipo de consulta/problema

Nivel bajo de consulta, soporte tecnico esencial y resolución rápida de problemas

\section{Nivel 2 \\ 15 minutos a 24 horas}

Problemas de mayor complejidad que no logren requerir desarrollo por parte del encargado

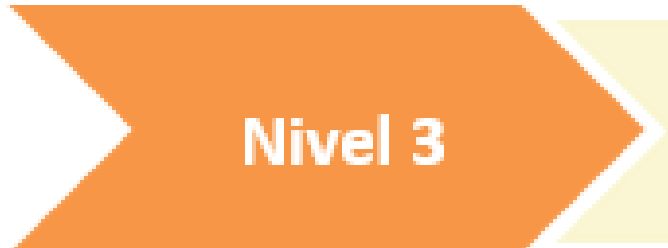

24 horas a 360 dias

Problemas

identificados que requieren desarrollo por parte del equipo

Figuro 1. Niveles de soporte. Adaptado de ITIL ${ }^{\circ}$ V3: Entender el enfoque y adoptar las buenas prácticas

\section{GESTIÓN DE INCIDENTES}

En la gestión de incidentes se detallan las acciones a tomar cuando ocurre un incidente de diversas índoles se materializa, enfatizando en brindar una respuesta rápida y eficaz.

\section{OBJETIVOS DE LA GESTIÓN DE INCIDENTES}

Los objetivos principales de la Gestión de Incidentes son:

- Detectar incidentes que alteren el funcionamiento normal del sistema.

- Registrar, clasificar y re direccionar estos incidentes al responsable pertinente, según la clasificación brindada en el punto anterior.

\section{BENEFICIOS DE LA GESTIÓN DE INCIDENTES}

Los principales beneficios de una correcta Gestión de Incidentes incluyen:

- Mejorar la productividad de los usuarios.

- Monitoreo constante de desempeño de procesos y revisión de funcionamiento óptimo.

- Reducir el tiempo perdido por incidentes del sistema.

- Mejorar la percepción respecto al desempeño del sistema.

- Resolución de incidentes de manera rápida, aumentando la calidad del producto.

\section{RIESGOS DE UNA INCORRECTA GESTIÓN DE INCIDENCIAS}

Una gestión incorrecta de incidentes puede derivar en:

- Reducción del tiempo útil de operaciones.

- Responsabilidades asignadas incorrectamente. 
- Sensación de respuesta ineficiente frente a incidentes.

- Sistema considerado ineficiente e inútil.

\section{REGISTRO DE INCIDENTES}

El registro de incidentes se realizará conforme se vayan identificando incidencias. Todo tipo de incidencias se registrarán a través de un servicio de HelpDesk|gestionado por la misma empresa, posterior a este registro, se re direccionará al responsable pertinente en base a la lista presentada anteriormente.

\section{CONSIDERACIONES}

- La admisión del incidente: El personal de HelpDesk debe evaluar el incidente presentado para la asignación correcta hacia el responsable, considerando las caracteristicas del incidente presentado.

- Validación de incidente: Es necesario validar que se trate de un incidente real y no de un error de usuario.

- Repetición de incidente: Es necesario validar que se trate de un incidente nuevo y no uno ya reportado. Para este punto es necesario considerar que si se trata del mismo error en distintos módulos, se debe considerar como incidencias distintas.

- Asignación de referencia: El incidente tendrá asignada una referencia que le identificará únicamente y servirá como identificador único para la comunicación entre el cliente y el personal de soporte

- Información adicional: Se remitirá un formato a llenar con información adicional del incidente, disponible en el anexo: Formato de Registro de Problema - HelpDesk

\section{CLASIFICACIÓN DE INCIDENCIAS}

La clasificación de incidentes es esencial para distribuir la resolución de los mismos en base a los roles asignados. Las siguientes consideraciones deben ser tomadas en cuenta para una adecuada clasificación de incidentes.

- Categorización: Se categoriza en base al tipo de incidente y al módulo perteneciente, cruzando de esta manera la identificación del servicio o servicios afectados.

- Prioridad: se establece la prioridad, dependiendo del impacto y urgencia de la misma, determinada por la conexión del error con la operatividad del sistema.

- Asignación de recursos: La asignación de recursos es según se determine el impacto y prioridad de resolución del incidente

- Comunicación constante: se comunica al usuario cuando el cliente ha sido solucionado y cuando está siendo revisado por el personal calificado.

ANÁLISIS, RESOLUCIÓN Y CIERRE DE INCIDENTES

El análisis del incidente comienza por una revisión de registros históricos que permita determinar si se trata de un incidente reportado con anterioridad. En caso no lo sea, se delega la resolución del incidente a quien se considere responsable en base al impacto que tendría la solución del mismo, según lo identificado anteriormente.

\section{GESTIÓN DE PROBLEMAS}

La gestión de problemas permite identificar las causas subyacentes que generen una interrupción o alteración al servicio ofrecido, buscando además maneras de resolverlo. La gestión de problemas puede tener un enfoque reactivo, siendo aplicada cuando se identifique una incidencia o proactiva, siendo identificada la incidencia por el propio analista encargado de la identificación de problemas en el uso diario de la herramienta. 


\section{CONTROL DE PROBLEMAS}

Para el control de problemas, es necesario seguir los siguientes pasos:

- Identificación del problema: Se identifica el problema presentado por el usuario y se revisa si esto se ha presentado con anterioridad, con el fin de identificar una posible solución o si se trata de un error de usuario. Para esto se cuenta con un registro de incidencias y con un registro de monitoreo de desempeño del sistema, habiendo muchas veces caídas de servicios de $\mathrm{Tl}$ por interrupciones o intermitencias dentro de la plataforma subyacente al sistema.

- Identificación de solución: En base al problema identificado, se identifican las posibles causas y se realizan pruebas respectivas, tanto de manera a futuro como hacia atrás (a través de pruebas de regresión], con el fin de identificar que solución brinda la respuesta necesaria para la incidencia resaltada.

- Aplicación de solución: se aplica la solución cuando se haya identificado la misma y su no interferencia con otros aplicativos o interrupción de otros servicios. En caso sea necesario interrumpir servicios o el sistema, se debe realizar en horas donde no afecte al negocio y considerando que las soluciones deben ser probadas exhaustivamente para que no tengan impacto a las aplicaciones subyacentes que comparten la misma plataforma.

- Registro de problema y solución: se actualiza el inventariado de incidencias y soluciones, detallando quién la reporto, el módulo y el(los) servicio(s) afectado(s).

CLASIFICACIÓN Y ANÁLISIS DE RECURSOS

El problema se puede clasificar de manera que se mantenga un repositorio actualizado y legible de las incidencias y problemas encontrados, empleando categorias en base a sus causas, tales como problemas de hardware, lógicas de aplicativo o compatibilidad, además de mostrar las incidencias por módulo y por área que lo reportó.

Para la asignación de recursos encargados de mitigar los problemas presentados, es necesario identificar la prioridad del problema en base al impacto que tenga respecto al giro del negocio, siendo los aplicativos comerciales y logísticos los de mayor criticidad según lo identificado en las fases de análisis de negocio. Una vez identificada la prioridad, es necesario identificar el tipo de solución a brindar para determinar el plazo que se tomará y los recursos que serán asignados a la mitigación de este problema.

ANÁLISIS Y DIAGNÓSTICOS

El análisis del problema se realizará en base a lo presentado por el usuario a través del portal de soporte, donde se pedirá un alto nivel de detalle y además el llenado del formulario Formato de Registro de Problema - HelpDesk. En el análisis se buscará:

- Determinar las causas del problema reportado

- Descartar posibles fuentes de error

- Identificar errores/catalizadores potenciales

Una vez se realice este análisis, se pasará a diagnosticar la solución y a derivarla al personal respectivo, informando al usuario del tiempo a esperar.

\section{CONTROL DE ERRORES}

Una vez identificado el error y la solución, es necesario mantener un registro actualizado del mismo notificando su criticidad, el módulo y servicio afectado, la causa, la solución y la fecha en que se identificó, con el propósito de mantener una base de datos actualizada de errores que permite la pronta mitigación de eventuales errores futuros.

En caso se trate de una solución de nivel 3, de amplia demora a un aplicativo de gran impacto, es necesario determinar con el administrador de TI en coordinación con la Gerencia si se tomará el camino de acoplar la 
solución lo más rápido posible o demorarla a través de una solución temporal, siendo esto muchas veces lo recomendado por el alto costo y poco impacto que tienen determinados errores.

\section{GESTIÓN DE CAMBIOS}

Es necesario gestionar los cambios realizados en un sistema en producción con el propósito de mantener un histórico de los cambios e identificar el desempeño histórico en base a los mismos. Dentro de las posibles causas de cambios existen:

- Cambio de lógicas de negocio

- Cambios en normativas regales

- Cambios regulatorios de mercados

- Mejoras de aplicativo

- Necesidad de ypgrade de plataforma

En caso se trate de un cambio muy brusco, es necesario contemplar la necesidad de realizar un cambio total del sistema, es decir, dar un paso a retiro del mismo. El sistema ha sido desarrollado con un enfoque escalable $y$ adaptable, por lo que es necesario verificar la auténtica necesidad y motivación tras una posible necesidad de reemplazo del sistema.

En la gestión de cambios se buscará manejarlos de manera óptima y con el propósito que la información recopilada en cambios anteriores enriquezca la adopción de otros cambios a futuro, con el fin de enriquecer la capacidad del sistema. A continuación se detallarán los pasos a seguir para una adecuada gestión de cambios.

\section{REGISTRO}

Todo cambio debe ser registrado, explicando lo realizado, la motivación, el/los aprobadores del mismo y en caso se trate de un cambio que se revirtió, explicar la razón del mismo. Todo cambio de todo tipo será sujeto a un registro similar, con el fin de mantener una base de datos unificada $y$ enriquecida por las experiencias operacionales que se ha tenido. Todo cambio manejará un estado propio de la implementación del mismo, además del estado de la aprobación del cambo en caso sea necesario.

\section{CLASIFICACIÓN}

Posterior al registro del cambio, se establecerá una prioridad para el cambio en base al impacto del mismo en el flujo lógico del desarrollo del negocio, considerando también la cantidad de recursos necesarios para la adopción del cambio y el costo que este implicaría, de tratarse de un nuevo desarrollo o de un desarrollo que pueda involucrar a terceras partes. De esta manera también se realizará una categorización y se actualizará la base de conocimiento de cambios ejecutados o en proceso de ejecución. En este paso es posible desestimar cambios por su alto impacto e imposibilidad de ser abarcado en un tiempo cercano

\section{GESTIÓN DE NIVELES DE SERVICIO}

La gestión de niveles de servicio, según ITIL, busca poner la tecnología al servicio del cliente, siendo vista como un medio de agregar valor a la experiencia al usuario o cliente. Dentro de ITIL, se establece que la gestión de niveles de servicio debe velar por la calidad de servicios de $\mathrm{Tl}$ alineados a los procesos de negocio, según las necesidades de los usuarios. 
La gestión de niveles de servicios debe:

- Documentar los servicios de TI ofrecidos

- Reflejar los servicios de manera contrastada contra las necesidades de usuario en base a los procesos

- Establecer indicadores de TI para el desempeño de servicios

- Monitorear la calidad de los servicios con el objetivo de mejorarlos continuamente

La gestión de niveles de servicios es compuesta por los siguientes procesos reflejados en la siguiente imagen y explicados a continuación:

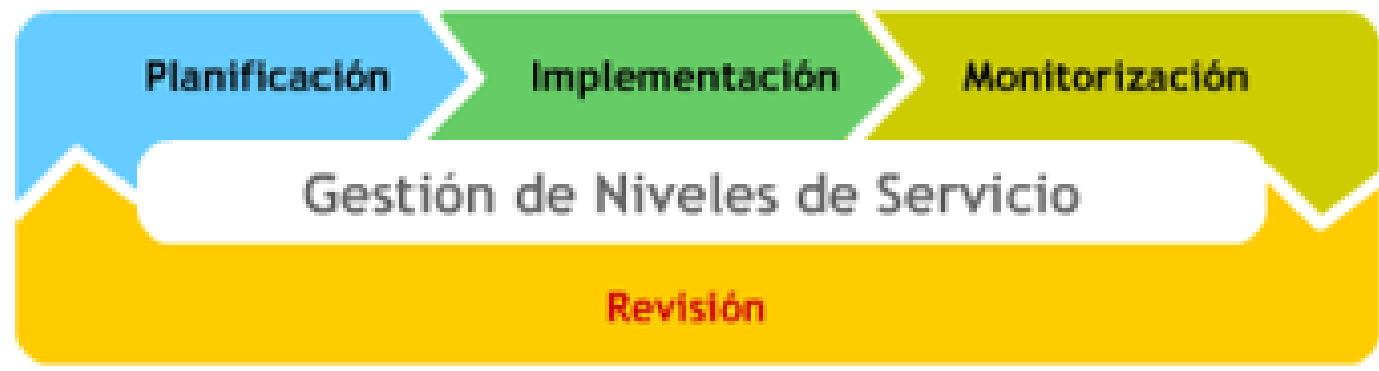

Figwa 2. Gestion de Niveles de Servicio. Adaptado de ITIL Va: Entender ell enfoque y adoptar las buenas prácticas

\section{PLANIFICACIÓN}

La planificación de la Gestión de Niveles de Servicio requiere que se realice un trabajo en conjunto por parte de los stakeholders del sistema y los componentes del mismo. Dentro de esta fase se realiza la asignación de recursos para el desarrollo del catálogo de servicios, el desarrollo del catálogo en sí, el desarrollo de los $5 \not \Delta s$, se plantean herramientas y métodos de monitoreo de performance y además se identifican las necesidades dell cliente.

\section{IMPLEMENTACIÓN}

La fase de implementación busca llegar a un punto común respecto a lo planificado $y$ a lo que será implementado, considerando factores de complejidad, costos y tiempo a la hora de realizar dicha implementación. En los acuerdos, tanto de nivel de servicio, operación y soporte, se debe especificar a gran detalle los limites acordados con el cliente, con el fin de evitar inconvenientes futuros.

\section{MONITORIZACIÓN}

El proceso de monitorización, muchas veces llamado supervisión, resulta nececario para plantear una mejora continua respecto a los servicios offecidos, realizando informes $y$ análisis de rendimiento, verificando la interacción con proveedores externos y planteando oportunidades de mejora a través de la elaboración de programas de mejora del servicio, también llamados s.p.

El proceso de monitorización de los niveles de servicio es imprescindible si queremos mejorar progresivamente los servicios asociados.

\section{REVISIÓN}

La revisión resulta necesaria para establecer los limites de cobertura de soporte brindados hacia el cliente, con el fin de lograr un acuerdo común que beneficie a ambas partes. se trata de un proceso continuo que busca identificar las mejores posibles a realizar en base a circunstancias identificables en el futuro. 


\section{GESTIÓN DE SEGURIDAD}

Respecto a la gestión de seguridad, es necesidad que esta vele por la integridad y salvaguarda de la información gestionada por la empresa, delimitando accesos para la misma en base al rol de cada persona en la empresa.

La gestión de seguridad busca:

- Establecer una política de seguridad en base a las necesidades y contexto del negocio.

- Gestionar óptimamente en base a buenas prácticas el control de acceso a información, abarcando modificación, visualización, lectura y tratamiento de la misma.

- Minimizar los riesgos que supongan una amenaza para la continuidad y operatividad del sistema y negocio.

La gestión de seguridad es una tarea a llevar a cabo en conjunto, por lo que es necesario demostrar el alineamiento de todas las partes interesadas a lo acordado bajo las políticas de seguridad.

\section{ESTABLECIMIENTO DE POLIITICAS DE SEGURIDAD}

Para una óptima gestión de la seguridad, es necesario establecer políticas de seguridad que sirvan como lineamiento para la gestión de la seguridad en el sistema. Es necesario que en asta política se determine la relación de la política de seguridad con la política general del negocio, su coordinación con procesos de $\mathrm{TI}_{\text {, }}$ tales como los anteriormente mencionados, los protocolos para solicitar acceso a información, los procedimientos de análisis de riesgos y el alcance del plan presentado.

\section{DEFICIÓN DEL PLAN DE SEGUURIDAD}

Para definir el plan de seguridad, es necesario identificar los requerimientos del negocio planteado, trabajando de manera colaborativa con la gestión de niveles de servicio, siendo esta la responsable de comunicar con ell cliente y gestionar su satisfacción con el sistema. Es además necesario identificar métricas e indicadores para evaluar los niveles de seguridad, siendo estos contrastables contra los presentados por diversos estándares de trascendencia global, tal como los dominios del anexo A dell 15027001.

\section{APLICACIÓN DEL PLAN DE SEGURIDAD}

La aplicación de las medidas de seguridad planificadas debe realizarse de manera inmediata conforme se haya determinado el plan de manera astable y con conformidad de la gerencia. Esta aplicación de medidas as responabilidad del administrador de TI en aplicarlas y se trata de una responsabilidad en conjunto reforzarlas y sugerir oportunidades de mejora de las mismas.

Es necesario que el personal se encuentre capacitado $y$ cumpla con las medidas, determinando la documentación referencial necesaria y además evitando que se obtengan accesos o permisos de maneras ajenas a las establecidas en la política de seguridad.

Es necesario además considerar los procesos que brindarán soporte a la seguridad de la información de la empresa, tales como la ejecución de copias de seguridad, detalladas a continuación: 
- Backups del Sistema Operativo: Es un backup sencillo pero de gran consumo de recursos. Es recomendable realizarlo esporádicamente, puesto que requiere parar la base de datos $y$ el servidor en el cual se está ejecutando.

- Backups en Frio: Los backups en frío implican detener la base de datos para realizar la copia hasta el punto en que se encontraba previo a la detención. Resulta complejo por la necesidad de detener la ejecución de la base de datos, que muchas veces es inconcebible aun en horas en que no se está trabajando, ya que se sigue procesando información.

- Backups en Caliente: El backup en caliente es una copia de seguridad realizada en plena operación de la base de datos, siendo esto complicado en caso se trate de una base de datos muy amplia, puesto que mellará ampliamente en la performance de la misma, siendo muchas veces recomendado parar la ejecución de la base de datos y tomarse un tiempo en sacar la copia de seguridad.

- Backups Lógicos con Export/Import: Los backups lógicos permiten hacer una copia en otro servidor de la base de datos ejecutándose. Muchas veces es usado para contextos de páginas web inmensas y se busca ahondar en la redundancia de información para evitar la pérdida de la misma.

La estrategia de copias de seguridad se determinará en base al alcance de las políticas de seguridad, considerando que las copias de seguridad en frío resultan las ideales para no mellar en temas de performance a la base de datos ni al servidor.

PRESENTACIÓN DE LA RECUPERACIÓN

La recuperación del sistema resulta compleja ya que se tiene que retroceder a un estado previo y realizar una carga masiva de información para subsanar algún detalle. Es necesario determinar un proceso para solicitar recuperación de información, siendo este gestionable a través de HelpDesk con las conformidades de la jefatura del operador que necesite la información. Dentro de los tipos de errores que se pueden presentar $y$ que requieran una restauración están:

- Pérdida de información por error humano

- Error de sentencias (consultas sin filtros aplicados)

- Fallos de procesos automáticos o de red

\section{GESTIÓN DE DISPONIBILIDAD}

El sistema se encuentra situado en un contexto web, siendo accesible a toda hora de manera interna $y$ remota. Es necesario gestionar la disponibilidad del mismo haciendo mediciones y estableciendo métricas que permitan identificar si el sistema se encuentra respondiendo de manera adecuada a las demandas en las horas cumbre de acceso, siendo estas generalmente las horas de oficina donde se usa el sistema de manera íntegra.

Es necesario además identificar posibles falencias de desempeño o funcionalidades, puesto que estas deben ser mitigadas a la brevedad posible para garantizar el óptimo desempeño de la herramienta.

Para el ámbito de la disponibilidad, resulta esencial abarcar los frentes de la disponibilidad del sistema en si y la disponibilidad de la plataforma subyacente al sistema, por lo que las métricas deben hacer mediciones respecto a ambos y tomar las medidas necesarias para garantizar que el usuario nunca se tope con un problema de rendimiento que sea previsible en el contexto de un uso normal del sistema. Asimismo, la disponibilidad debe ir de la mano con la gestión de seguridad, garantizando la disponibilidad a pesar que se realice un ataque hacia el sistema, permitiendo que la empresa continúe operando normalmente.

Finalmente, es necesario definir la disponibilidad y los requerimientos de la misma en base al contexto de negocio. 


\section{ANEXO 20: CARTERA DE PROYECTOS}

\section{CARTERA DE PROYECTOS}

El presente documento contiene el plan de continuidad: Cartera de Proyectos del proyecto: Modelo de migración a la nube de los servidores de un data center. El siguiente contiene mejoras en el modelo propuesto, que por limitante de tiempo o financiamiento no se llegaron a concluir. Estos proyectos afectarían en las evoluciones que pueda llegar a tener el modelo, después de la implementación $y$ validación de este.

\section{OBJETIVO}

El objetivo de la cartera es brindar mejoras del modelo propuesto, mejoras que por motivos de tiempo $\gamma$ financiamiento no se llegaron a concretar en este proyecto. También estas mejoras se dan por la evolución de la tecnología y nuevas tendencias que puedan salir en el mercado. Asimismo con estas mejoras que se puedan dar en el tiempo, brinda una automatización en la migración y de esa forma tener mejores resultados.

\section{PROYECTOS}

\section{PROYECTO I}

\begin{tabular}{|l|l|}
\hline PROBLEMA & $\begin{array}{l}\text { El crecimiento exponencial del uso de microservicios (contenedores) no es } \\
\text { proporcional con la escalabilidad de los recursos del data center. }\end{array}$ \\
\hline CAUSA & $\begin{array}{r}\text { Las causas de la retención del crecimiento del data center son las siguientes: } \\
\text { - Degradación de servidores }\end{array}$ \\
& $\begin{array}{l}\text { Altos costos de mantenimiento de servidores } \\
\text { Alto consumo de energía }\end{array}$ \\
\hline COLecimiento relevante del uso de microservicios
\end{tabular}


FUENTES DE INVESTIGACIÓN PROPUESTAS
Las fuentes de investigación propuestas para el modelo son las siguientes:

1. Voyager: Complete Cqutainer State Migration

1.1. Fuente: http://sci-hub.io/10.1109/icdcs.2017.91

1.2. Autores: Shriead Nadgowda, Sabil Suneia, Nilten Bila y Canturk Isci.

1.3. Resumen: Voyager es un servicio de migración de contenedor en vivo, diseñado de acuerdo con la Iniciativa de Principios de Contenedor Abierto. voyager es un novedoso servicio de migración independiente del proveedor que proporciona una migración consistente del sistema completo. Se monta a minimizar el tiempo de inactividad de la migración con una vista de unión de datos entre los hosts fuente y destino.

2. Cimplifie: Automaticallx Bebleatiog Containers

2.1. Fuente: http://sci-hub.io/10.1145/3106237.3106271

2.2. Autores: Vaikhav Bastogi, Rreuk Ravidsed, Lorenzo De Carli, Semesh tha $y$ Patrick MSParial

2.3. Resumen: Debido a las malas prácticas se acumula data innecesaria y se crean contenedores de tamaños excesivos. Estas prácticas aumentan innecesariamente el tamaño del contenedor. Para ello se proponen algoritmos y una herramienta llamada cimplifier, que abordar estas preocupaciones, dado un contenedor y simple definido por las restricciones de usuario, la herramienta lo divide en contenedores más simples, que están aislados unos de otros, solo se comunican según sea necesario, y solo incluye suficientes recursos para realizar su funcionalidad. Cimalifier conserva la funcionalidad original, conduce a la reducción en tamaño de imagen de hasta $95 \%$, y procesa incluso contenedores grandes en menos de treinta segundos.

3. A Review of Existing Cloud Automation Tools

3.1. Fuente:

https://innovareacademics.in/iournals/index.php/ajpcr/article/view/2051 $9 / 11878$

3.2. Autores: Prassana J, Anjali R Pawar y Neelanaraxanan $\mathrm{V}$.

3.3. Resumen: Muchas empresas ejecutan aplicaciones distribuidas en sus servidores en sus instalaciones. Sin embargo, si la carga en esos servidores cambia inesperadamente, entonces se vuelve tedioso. Por lo que requiere horas hombres para manejar tales situaciones, esto aumenta el gasto de capital. Por lo tanto, muchas empresas han comenzado a migrar sus servidores a la nube. Esta migración de las aplicaciones a la nube es una de los principales desafios. Para configurar $y$ administrar la creciente infraestructura compleja, después de migrar estas aplicaciones a la nube es realmente una tarea que consume mucho tiempo y proceso tedioso que resulta en tiempo de inactividad. Por lo tanto, se necesita automatizar este entorno. Para lograr se propone herramientas como Tercaforcu y formación de nubes para la automatización de infraestructuras y Rocker. and Hábitat para la automatización de aplicaciones. 


\section{PROYECTO II}

\begin{tabular}{|c|c|}
\hline PROBLEMA & $\begin{array}{l}\text { A pesar que cloud es un habilitador clave para la transformación digital, el presente } \\
\text { modelo no implica la migración a la transformación digital de la organización, la cual } \\
\text { permite automatizar procesos, mayor satisfacción del usuario, herramientas de toma de } \\
\text { decisiones, entre otros que permiten incrementar la rentabilidad de la organización. } \\
\text { Las organizaciones (especialmente las PYMES) en su mayoria no han adoptado la } \\
\text { transformación digital. }\end{array}$ \\
\hline CAUSA & $\begin{array}{l}\text { Las causas de que las PYMES no adopten la transformación digital son: } \\
\text { - Desconocimiento de cómo empezar a adoptar transformación digital. } \\
\text { - Descos costos de inversión. }\end{array}$ \\
\hline SOLUCIÓN & Modelo de integración de PYMES de la transformación digital basada en cloud \\
\hline $\begin{array}{l}\text { OBJETIVOS } \\
\text { PROPUESTOS }\end{array}$ & $\begin{array}{l}\text { OG: Elaborar un modelo de adaptación de transformación digital para PYMES. } \\
\text { - OE1: Analizar sobre la transformación digital y la adopción de esta. } \\
\text { - OE2: Diseñar un modelo de adopción de transformación digital para PYMES } \\
\text { - OE3: Validar el modelo de adopción de transformación digital para PYMES } \\
\text { - OE4: Proponer un plan de continuidad que asegure la funcionalidad del modelo }\end{array}$ \\
\hline $\begin{array}{l}\text { FUENTES DE } \\
\text { INVESTIGACIÓN } \\
\text { PROPUESTAS }\end{array}$ & $\begin{array}{l}\text { Las fuentes de investigación propuestas para el modelo son las siguientes: } \\
\text { 1. Digital Business Ecosystem Transformation - Towards Cloud Integration } \\
\text { 1.1. Fuente: http://sci-hub.cc/10.1109/hicss.2016.491 } \\
\text { 1.2. Autores: Kaci Korpela, Karci Mikkgnen, Jukka Hallikas y Mikka. Exocönea } \\
\text { 1.3. Resumen: Los ecosistemas empresariales digitales se están convirtiendo en un } \\
\text { concepto cada vez más popular como un entorno abierto para modelar y } \\
\text { construir una integración de sistema interoperable. Las organizaciones } \\
\text { empresariales se han dado cuenta de la importancia de utilizar estándares como } \\
\text { un método rentable para acelerar la integración de los procesos comerciales. La } \\
\text { participación de las pequeñas y medianas empresas (pYME) en el comercio } \\
\text { mundial está aumentando; sin embargo, las transacciones digitales todavía están } \\
\text { en un nivel bajo. Se espera que la integración en la nube ofrezca un modelo } \\
\text { comercial rentable para formar una cadena de suministro digital interoperable. Al } \\
\text { observar los modelos de integración, podemos identificar el gran potencial de los } \\
\text { servicios en la nube para acelerar la integración. Se lleva a cabo un estudio de } \\
\text { caso industrial. } \\
\text { Este documento investiga y aporta nuevos conocimientos sobre cómo un enfoque } \\
\text { descendente mediante el uso de un marco de ecosistema empresarial digital } \\
\text { permite a los gerentes de negocios definir nuevos requisitos y funcionalidades } \\
\text { para la integración del sistema. A través del análisis, identificamos el límite actual } \\
\text { de diseño de integración. Usando el marco de clusteriog en la nube, identificamos } \\
\text { cómo el diseño afecta los servicios de integración en la nube. }\end{array}$ \\
\hline
\end{tabular}


2. When Energy Revolution Meets Digital Transformation

2.1. Fuente: https://sci-hub.cc/10.1007/978-3-319-59424-8 7

2.2. Autores: Shébérazade Benzerga, Reminik Hauf, Michael Bretz y Ahmed Bqunfour

2.3. Resumen: Se presenta la tendencia de Economía Digital para comprender mejor en qué medida esta tendencia podría ser clave para el futuro de las industrias en el nivel macro. Al igual se toca la definición de transformación digital. Sus dimensiones serán abordadas para comprender mejor los impactos estratégicos inherentes. Su caso de uso sera viniendo de una planta en una empresa de fabricación, entonces, el caso específico de la energía se analizará la eficiencia, que está respaldada por una innovación de proceso patentada basado en tecnologías digitales, como algoritmos de autoaprendizaje $\gamma$, por tanto, en un aumento del papel de las $\mathrm{TI}$ en el área de producción.

3. Working Life Within a Hybrid World - How Digital Transformation and Agile Structures Affect Human Functions and Increase Quality of Work and Business Performance

3.1. Fuente: https://sci-hub.io/10.1007/978-3-319-60372-8 1

3.2. Autores: Wilhelm Bauer, Sebastian Schluad y christian Vocke

3.3. Resumen: El trabajo se está volviendo cada vez más digital y se verá bastante diferente de lo esperado hoy. Combinando el físico

y el mundo virtual representa el factor de éxito clave para el trabajo futuro. Los estudios examinan cómo la digitalización va a penetrar la vida laboral más adelante, mostrando medidas centrales y soluciones seleccionadas para la organización. como estructuras, las necesidades de cualificación humana y las condiciones de trabajo optimizadas en un mundo híbrido, dando lugar a cambios masivos con referencia a todas las dimensiones de trabajo. Como nuevos requisitos relacionados con los objetivos de trabajo, las tareas de trabajo, el trabajo en equipo, espacio de trabajo y nuevos desafíos para la organización, calificación, el empleo y el liderazgo surgen.

4. Automated business process management - in times of digital transformation using machine learning or artificial intelligence

4.1. Fuente: http://sci-hub.io/10.1051/matecconf/201712104007

4.2. Autores: Daniel Paschek, Gaius Tudor Luminossu y Anca Rraghici

4.3. Resumen: La optimización continua de los procesos de negocio sigue siendo un desafío para las empresas. En tiempos de transformación digital, cambio más rápido, las condiciones internas y externas y nuevas expectativas del cliente para una entrega más rápida y la mejor calidad de productos y muchas más, las empresas deben configurar su proceso interno de la mejor manera. El propósito es analizar cómo la transformación digital tendrá un impacto en el proceso empresarial Administración (BPM) al usar métodos como aprendizaje automático o inteligencia artificial. Por lo tanto, se explicarán los componentes centrales, comparado y establecido en relación. Para identificar entrevistas de áreas de aplicación y el análisis se retrasará con las empresas digitales. Esto será una recomendación para la acción en el campo de BPM y proceso de optimización a través del aprendizaje automático y la inteligencia artificial. Los enfoques de optimización y procesos de gestión a través del aprendizaje automático y la inteligencia artificial ayudará a las empresas a decidir qué herramienta sería el mejor para un BPM automatizado. 


\section{PROYECTO III}

\begin{tabular}{|c|c|}
\hline PROBLEMA & $\begin{array}{l}\text { Poder garantizar un adecuado nivel de servicio y gestionar la calidad a través de } \\
\text { dimensiones tales como el rendimiento, la disponibilidad y la fiabilidad que garanticen la } \\
\text { fiabilidad del modelo de calidad. }\end{array}$ \\
\hline CAUSA & $\begin{array}{l}\text { No poder identificar los atributos de calidad necesarios para garantizar los servicios cloud } \\
\text { en las empresas peruanas. }\end{array}$ \\
\hline SOLUCIÓN & Modelo de calidad de servicios cloud para PYMES peruanas. \\
\hline $\begin{array}{l}\text { OBJETIVOS } \\
\text { PROPUESTOS }\end{array}$ & $\begin{array}{l}\text { OG: Elaborar un modelo de calidad de servicios cloud. } \\
\text { - OE1: Analizar sobre los servicios cloud y métricas de calidad. } \\
\text { - OEz: Diseñar un modelo de calidad de servicios cloud. } \\
\text { - OE3: Validar el modelo de calidad de servicios cloud. } \\
\text { - OE4: Proponer un plan de continuidad que asegure la funcionalidad del modelo }\end{array}$ \\
\hline $\begin{array}{l}\text { FUENTES DE } \\
\text { INVESTIGACIÓN } \\
\text { PROPUESTAS }\end{array}$ & 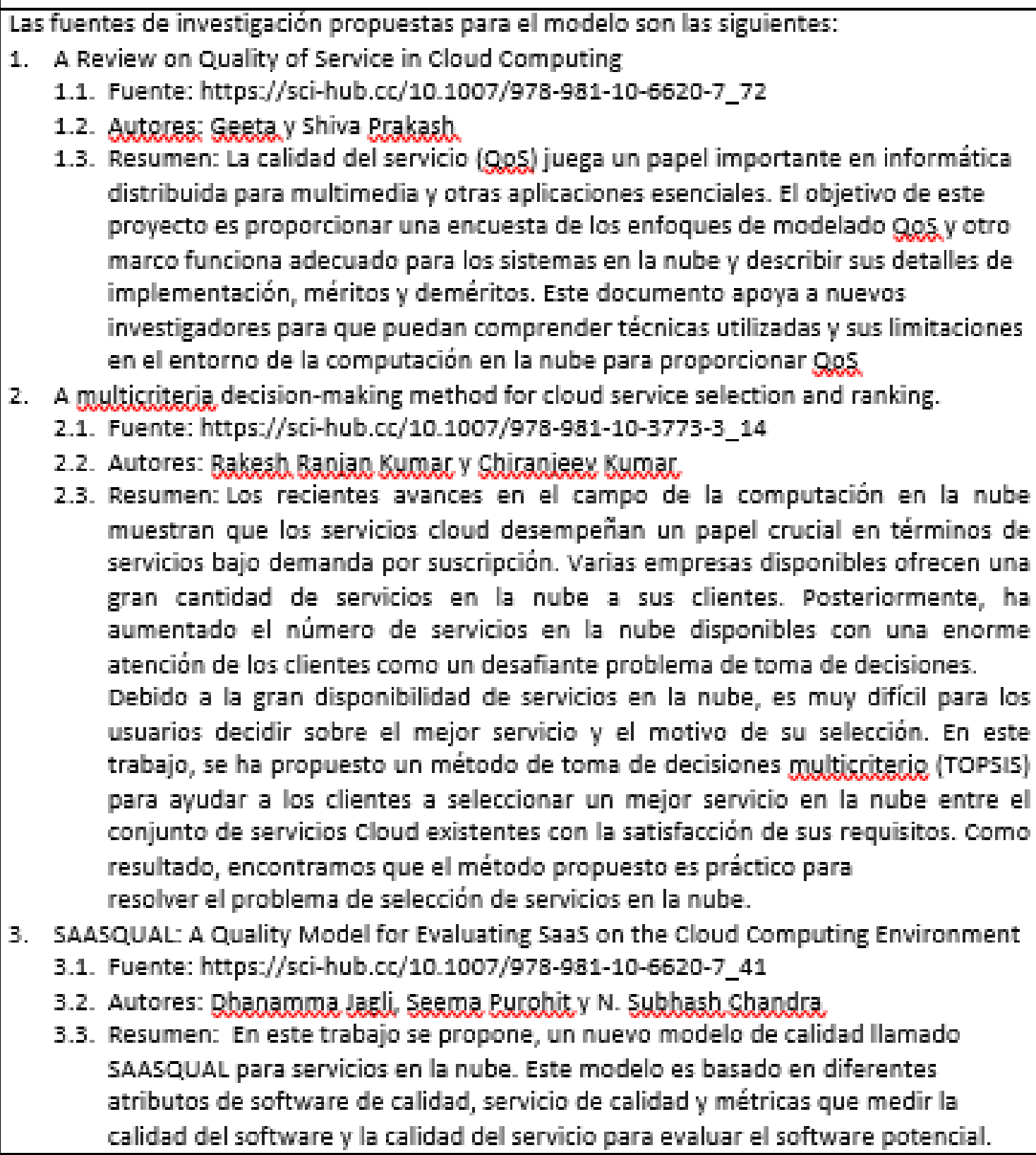 \\
\hline
\end{tabular}




\section{ANEXO 21: CERTIFICADO IT SERVICE}

\section{Certificado IT SERVICE}

A

Modelo de Migración a la Nube de los Servidores de un Data Center

Por su cumplimiento de las características de Calidad de ISO 9126:

Funcionalidad y Usabilidad

Autor:

Loo Cuya, Fabiola

Rojas Solórzano, Christian

Otorgado el 07 de Noviembre de 2017

ITSERVICE014-20172 


\section{ANEXO 22: CERTIFICADO DATA CENTER}

\section{CONSTANCIA DE DESPLIEGUE EN PRODUCCIÓN}

\section{Empresa Datacenter}

Modelo de migración a la nube de los servidores de un Data Center

\section{Integrante 1 Loo Cuya Fabiola Magaly Integrante 2 Rojas Solórzano Christian Gianfranco}

Este documento certifica que la aplicación está desplegada en los servidores de la empresa DATACENTER validado por los integrantes del proyecto y supervisado por el gestor de servicios de la empresa prestadora del servicio.
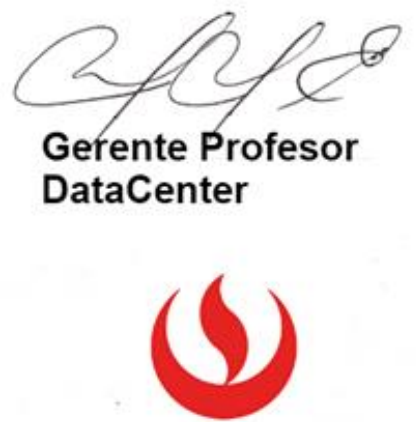


\section{ACTA DE ACEPTACIÓN Y CIERRE DE PROYECTO}

\section{SECCIÓN 1. INFORMACIÓN GENERAL}

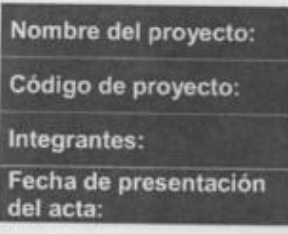

\section{Modelo de Migración a la nube de los servidores de un data center} MMNSDC

Fabiola Loo

Christian Rojas

$16 / 11 / 2017$

\section{SECCIÓN 2. DETALLES DE CIERRE DE PROYECTO}

\begin{tabular}{|c|c|c|c|c|}
\hline Id & Puntos De Control & $\begin{array}{l}\text { Fecha de } \\
\text { Aprobación }\end{array}$ & $\begin{array}{l}\text { Responsable de } \\
\text { Aprobación }\end{array}$ & Comentarios \\
\hline \multirow[t]{12}{*}{1} & Control de Entregables del Proyecto & & & \\
\hline & $\begin{array}{l}\text { Entregable OE1: Buenas Practicas de la migración a } \\
\text { la nube }\end{array}$ & 01/07/2017 & Edgar Diaz & - \\
\hline & $\begin{array}{l}\text { Entregable OE2: Modelo de migración a la nube de } \\
\text { los servidores de un data center }\end{array}$ & 08/01/2017 & Edgar Diaz & $\cdot$ \\
\hline & $\begin{array}{l}\text { Entregable OE3: Business Principles, Goals and } \\
\text { Drivers }\end{array}$ & $25 / 09 / 2017$ & Edgar Diaz & - \\
\hline & Entregable OE3: Current IT Enviroment & $25 / 09 / 2017$ & Edgar Diaz & $\cdot$ \\
\hline & Entregable OE3: Especificación de Requerimientos & $25 / 09 / 2017$ & Edgar Diaz & $\cdot$ \\
\hline & $\begin{array}{l}\text { Entregable OE3: Benchmarking de Plataformas } \\
\text { Cloud }\end{array}$ & $25 / 09 / 2017$ & Edgar Diaz & $\cdot$ \\
\hline & Entregable OE3: Estudio de Viabilidad & $25 / 09 / 2017$ & Edgar Diaz & - \\
\hline & Entregable OE3: Documento de Implementación & $25 / 09 / 2017$ & Edgar Diaz & $\cdot$ \\
\hline & Entregable OE4: Plan de continuidad & $11 / 11 / 2017$ & Edgar Diaz & - \\
\hline & Entregable OE4: Plan de Soporte & $11 / 11 / 2017$ & Edgar Diaz & $\cdot$ \\
\hline & Entregable OE4: Cartera de Proyectos & $11 / 11 / 2017$ & Edgar Diaz & - \\
\hline \multirow[t]{5}{*}{2} & Control de Archivo Documental & & & \\
\hline & Acta de Aprobación OE1 & 01/07/2017 & Edgar Diaz & - \\
\hline & Acta de Aprobación OE2 & $08 / 07 / 2017$ & Edgar Diaz & - \\
\hline & Acta de Aprobación OE3 & $25 / 09 / 2017$ & Edgar Diaz & - \\
\hline & Acta de Aprobación OE4 & $11 / 11 / 2017$ & Edgar Diaz & - \\
\hline
\end{tabular}

\section{ANEXO 23: ACTA DE CIERRE DEL PROYECTO}




\section{SECCIÓN 3. CONCLUSIONES FINALES}

Los entregables se realizaron con seguimiento del cliente.

El modelo propuesto se implementó en la empresa Globokas Perú.

Se concluyó satisfactoriamente el proyecto.

SECCIÓN 4. APROBADORES

\begin{tabular}{|l|l|l|l|}
\hline No & Nombres y Apellidos & Entidad / Cargo \\
\hline 1 & Edgar Diaz Amaya & Profesor Cliente & Firma \\
\hline & & & \\
\hline
\end{tabular}

